\title{
A Virtual Ocean framework for environmentally adaptive, embedded acoustic navigation on autonomous underwater vehicles
}

by

\author{
EeShan Chetan Bhatt \\ B.S.E. 2015, Mechanical Engineering, Duke University \\ Submitted to the Department of Mechanical Engineering \\ and the Joint Program in Applied Ocean Science and Engineering \\ in partial fulfillment of the requirements for the degree of Doctor of Philosophy \\ at the \\ Massachusetts Institute of Technology \\ and the \\ Woods Hole Oceanographic Institution
}

September 2021

๑) 2021 EeShan Bhatt. All rights reserved.

The author hereby grants to MIT and WHOI permission to reproduce and to distribute publicly

paper and electronic copies of this thesis document in whole or in part in any medium now known or hereafter created.

Author

Department of Mechanical Engineering, MIT

Applied Ocean Science \& Engineering, WHOI

July $7^{\text {th }} 2021$

Certified by

Henrik Schmidt

Professor of Mechanical and Ocean Engineering, MIT

Thesis Supervisor

Accepted by

Nicolas Hadjiconstantinou

Professor of Mechanical Engineering, MIT

Chair, Department Committee on Graduate Students

Accepted by

David Ralston

Associate Scientist with Tenure, Applied Ocean Physics \& Engineering, WHOI

Chair, Joint Committee for Applied Ocean Science \& Engineering 


\title{
A Virtual Ocean framework for environmentally adaptive, embedded
}

\section{acoustic navigation on autonomous underwater vehicles}

by

\author{
EeShan Chetan Bhatt
}

\begin{abstract}
Submitted to the Department of Mechanical Engineering and the Joint Program in Applied Ocean Science and Engineering on July $7^{\text {th }} 2021$ in partial fulfillment of the requirements for the degree of Doctor of Philosophy
\end{abstract}

\section{Abstract}

Autonomous underwater vehicles (AUVs) are an increasingly capable robotic platform, with embedded acoustic sensing to facilitate navigation, communication, and collaboration. The global positioning system (GPS), ubiquitous for air- and terrestrial-based drones, cannot position a submerged AUV. Current methods for acoustic underwater navigation employ a deterministic sound speed to convert recorded travel time into range. In acoustically complex propagation environments, however, accurate navigation is predicated on how the sound speed structure affects propagation. The Arctic's Beaufort Gyre provides an excellent case study for this relationship via the Beaufort Lens, a recently observed influx of warm Pacific water that forms a widespread yet variable sound speed lens throughout the gyre. At short ranges, the lens intensifies multipath propagation and creates a dramatic shadow zone, deteriorating acoustic communication and navigation performance. The Arctic also poses the additional operational challenge of an ice-covered, GPSdenied environment.

This dissertation demonstrates a framework for a physics-based, model-aided, real-time conversion of recorded travel time into range-the first of its kind-which was essential to the successful AUV deployment and recovery in the Beaufort Sea, in March 2020. There are three nominal steps. First, we investigate the spatio-temporal variability of the Beaufort Lens. Second, we design a human-in-the-loop graphical decision-making framework to encode desired sound speed profile information into a lightweight, digital acoustic message for onboard navigation and communication. Lastly, we embed a stochastic, ray-based prediction of the group velocity as a function of extrapolated source and receiver locations. This framework is further validated by transmissions among GPS-aided modem buoys and improved upon to rival GPS accuracy and surpass GPS precision.

The Arctic is one of the most sensitive regions to climate change, and as warmer surface temperatures and shrinking sea ice extent continue to deviate from historical conditions, the region will become more accessible and navigable. Underwater robotic platforms to monitor these environmental changes, along with the inevitable rise in human traffic related to trade, fishing, tourism, and military activity, are paramount to coupling national security with international climate security.

Thesis Supervisor: Henrik Schmidt

Title: Professor of Mechanical and Ocean Engineering 


\section{Paper Boats}

Day by day I float my paper boats one by one down the running stream.

In big black letters I write my name on them and the name of the village where I live.

I hope that someone in some strange land will find them and know who I am.

I load my little boats with shiuli flowers from our garden, and hope that these blooms of the dawn will be carried safely to land in the night.

I launch my paper boats and look up into the sky and see the little clouds setting their white bulging sails.

I know not what playmate of mine in the sky sends them down the air to race with my boats!

When night comes I bury my face in my arms and dream that my paper boats float on and on under the midnight stars.

The fairies of sleep are sailing in them, and the lading is their baskets full of dreams.

Rabindranath Tagore (1861-1941) 


\section{LAND ACKNOWLEDGMENT}

The ability to create this dissertation, in Cambridge and Falmouth, Massachusetts, at the MITWHOI Joint Program, is predicated on the dispossession and genocide of the Wampanoag Nation, who lived and continue to live with this land. This land, which I have arrived and settled on over the past six years, is the traditional unceded territory of the Wampanoag Nation. I recognize the painful history of genocide and forced occupation of these territories, and respect the many diverse Indigenous peoples connected to all these lands on which we gather from time immemorial. ${ }^{1}$

A year after its chartering, MIT became a land grant university through the Morrill Land Grant Act of 1862, and sold approximately 121 thousand acres of federally expropriated land from numerous Native nations to construct its earliest academic buildings in Boston. I am grateful for reporting from High Country News to educate me on this topic and encourage the reader to explore their database ${ }^{2}$.

If you would like to learn more about land acknowledgements, I benefited from this guide by the Native Governance Center. ${ }^{3}$ I would also encourage all MIT community members to engage with the Institute Community \& Equity office, and attend public events by MIT's Indigenous student groups, the Native American Student Association (NASA) and the local chapter of the American Indian Science and Engineering Society. I am grateful to all those I met through these groups, particularly Alvin, Luke, and Nancy, for their compassion, wisdom, and friendship.

\footnotetext{
${ }^{1}$ Adapted from the MIT land acknowledgement from MIT's Native American Student Association

${ }^{2}$ Robert Lee, "Morrill Act of 1862 Indigenous Land Parcels Database," High Country News, March 2020.

${ }^{3}$ https://nativegov.org/a-guide-to-indigenous-land-acknowledgment/
} 


\section{ACKNOWLEDGMENTS}

This section is a joyfully Sisyphean task-gratitude is life-long and life-changing. I will do my best to center the sense of belonging I felt while in the Joint Program, which was instrumental for me to feel like I could earn a PhD.

That sense of belonging begins with my advisor, Henrik. He has given me the space to figure things out for myself and to do them in my own way. More importantly, he has been a tremendous role model, as a scientist/engineer, a teacher, and a person. In many ways, given his leadership of the Laboratory for Autonomous Marine Sensing Systems (LAMSS), Henrik has served as both conductor and composer. My work is not possible without the rest of the LAMSS orchestra as well, especially for their contributions to the LAMSS software project. In particular, Oscar, Rui, Bradli, Dan, and Scott have been tremendous collaborators and friends, and essential to why graduate school was a growth-mindset atmosphere.

My committee, Andone and Pierre, have a knack for nicely asking questions to both highlight gaps in details and fill in the bigger picture. While giving that constructive feedback, they always conveyed that I could rise to the challenge. I am thankful that committee meetings were largely times of reflection and (tough) fun.

There are many WHOI faculty, scientists, and engineers who have contributed to this work through their teaching and mentoring-Caroline, Sam, Erin, Britt, Steve, YT Lin, Art (Newhall), Tim, Hanu, Bob, Art (Baggeroer), and Gwyneth.

For those who know me well, it will come as no surprise that I perhaps directed as much energy towards my research as I did towards my community, primarily on normalizing discussions about equity in ocean science (and academic institutions writ large) and inculcating a sense of belonging and advocacy as community standards. This is certainly what I am most proud of from my time in graduate school.

It has been such a pleasure to have worked alongside the folks who run the JP, Kris, Lea, Julia, and Meg, as well as those in MIT's Institute Community \& Equity Office, Rachel, Beatriz, and John.

And I am infinitely grateful to all the students who joined me in making some "good trouble"-it is beyond a doubt unreasonable to list all their names here, and that is its own blessing. But to roll the stone up the hill, I have so much love in my heart for Paris (P-Money), Chrissy, Justin, Rose, Mara, Henri, Lyssa, Bianca (Lepe), and Ufuoma, who have filled my cup in one way or another. I wish the best of luck to those who have picked up the mantle of the Joint Program's Applicant Support \& Knowledgebase (JP ASK)-Shawn, Katie, Max, Ciara, and Anna, as well as the 70+ students who signed up to be mentors. And I deeply cherish the JP tradition of naming of incoming cohorts, which started as a silly inside joke in my cohort (shoutout to some of my fellow Jellyfish, Ryan, Lizzie, Jen K, Rachel, Suzi, and Joleen)-may all the cohorts ahead be bequeathed and bequeath an official title!

For five out of the six years in my time in graduate school, I served as a Graduate Resident Advisor in Maseeh Hall. This brick castle, just across the street from my lab, has been my home. I never felt 
more MIT than when I was with my students or the house team. And to the "Muddy buddies" and "juice crew"-Yasmin, Nate, Sarah, Joseph, Cassandra, Bianca (Datta), Eric, and Jibril-I will always feel at home when any of us get together.

My wife, Shannon, has been a breadwinner and breadmaker over the past few years. And in addition to all that I love about our life together, I first (seriously) learned about marine science when we went to the Duke Marine Lab together, in the summer of 2013. So I owe her a life-changing moment, I guess? Add it to my tab.

I am grateful for the support provided by my parents (Prity \& Chetan), who quickly transitioned from confusion (what even is oceanography?) to excitement (check out this article!); by my in-laws (Kathy \& Tim), who have always been available as a sounding board; and by my rather large family, who have always cheered me on and showed up in droves for my Zoom defense. Graduate school, particularly finishing during the COVID-19 pandemic, has not been easy; having my family there brought an incredible sense of closure for this chapter of my life.

And I have the deepest, identity-affirming gratitude for the elders in my family-Nana, Nani, Dada Kaka, and Bhabhu-who walk on hallowed ground. This degree is as much theirs as it is mine.

And last, but certainly not least, I would like to thank you, the reader. It is more likely than not that I have something tangible to thank you for but ran out of space on this page. If not, now I do-thank you for reading. I would love to hear from you if you have any questions or collaborations in mind.

\section{Funding SOURCES}

This work was supported by:

Office of Naval Research (N00014-14-1-0214) -

GOATS'14 Adaptive and Collaborative Exploitation of 3-Dimensional Environmental Acoustics in Distributed Undersea Networks

Draper Laboratory Incorporated (SC001-0000001039) -

Positioning System for Deep Ocean Navigation (POSYDON)

Office of Naval Research (N00014-16-1-2129) -

MURI: The Information Content of Ocean Noise: Theory and Experiment

Office of Naval Research (N00014-17-1-2474) -

Environmentally Adaptive Acoustic Communication and Navigation in the New Arctic

Office of Naval Research (N00014-19-1-2716) -

TFO: Assessing Realism and Uncertainties in Navy Decision Aids

Department of Defense, Office of Naval Research -

National Defense, Science, and Engineering Graduate Fellowship 


\section{CONTENTS}

$\begin{array}{ll}\text { Contents } & 11\end{array}$

$\begin{array}{ll}\text { List of Figures } & 14\end{array}$

List of TABLes 17

$\begin{array}{ll}\text { ACRONYMS } & 18\end{array}$

1 INTRODUCTION 19

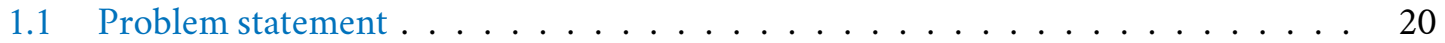

1.2 A new, blue Arctic . . . . . . . . . . . . . . . . . . 21

1.3 Underwater navigation and communication in the New Arctic . . . . . . . . . 23

1.4 Virtual Ocean approach . . . . . . . . . . . . . . . 25

1.5 Thesis organization $\&$ contributions $\ldots \ldots \ldots \ldots \ldots$

2 Background 29

2.1 Global Navigation Satellite System . . . . . . . . . . . . . . . . . . . 30

2.2 Underwater acoustic communication . . . . . . . . . . . . . . . 31

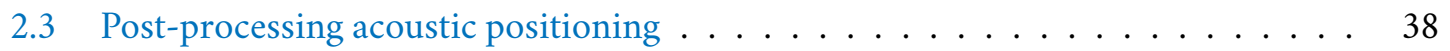

2.4 In situ acoustic positioning and navigation . . . . . . . . . . . . . . . . 44

2.5 Underwater vehicles and their autonomy $\ldots \ldots \ldots \ldots \ldots \ldots$ 
2.6 Environmentally adaptive autonomy . . . . . . . . . . . . . 56

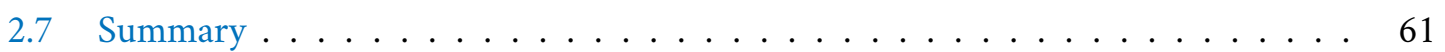

3 Strength and Variability of the Beaufort Lens 64

3.1 WHOI Ice Tethered Profilers $\ldots \ldots \ldots \ldots \ldots \ldots$

3.2 Data processing ........................ 66

3.3 Background on the Beaufort Sea . . . . . . . . . . . . 68

3.4 Duct strength parameter . . . . . . . . . . . . . . . . 73

3.5 Temporal analysis . . . . . . . . . . . . . . . . . . . 77

3.6 Spatial analysis . . . . . . . . . . . . . . . . . . . 83

3.7 Surface layer depth $\ldots \ldots \ldots \ldots \ldots \ldots$

3.8 Comparison to HYCOM \& historical data . . . . . . . . . . . . . . . . . . . 89

3.9 Discussion . . . . . . . . . . . . . . . . . . . . 91

4 Tactical Decision Aid framework for environmental adaptation 93

4.1 Background on Empirical Orthogonal Functions . . . . . . . . . . . . . . 95

4.2 Background on Tactical Decision Aids . . . . . . . . . . . . . . . . . . . 99

4.3 Generalized Virtual Ocean Framework ．. . . . . . . . . . . . . . . . 105

4.4 Execution of Framework for ICEX20 . . . . . . . . . . . . . . . . . . 110

4.5 Selected Results . . . . . . . . . . . . . . . . . . . . . . 126

4.6 Discussion . . . . . . . . . . . . . . . . . . . 131

5 Real-time acoustic Ray Range estimation during ICEX20 135

5.1 Integrated communication and navigation network $\ldots \ldots \ldots \ldots \ldots$

5.2 Modem experiment design . . . . . . . . . . . . . . . . . . . . . . 139

5.3 In situ group velocity estimation via minimal bounce criteria . . . . . . . . . . . 151

5.4 New group velocity estimation via nearest bounce criteria $\ldots \ldots \ldots$. . . . . . 155

5.5 In situ range estimation results from minimal bounce criteria . . . . . . . . . . . 157 
5.6 Post-processed range estimation results from nearest bounce criteria $\ldots$. . . . . . 159

5.7 Extended comparison between group velocity algorithms and sound speed environments .............................. 163

5.8 Limitations of GPS at polar latitudes . . . . . . . . . . . . . . . . . 174

5.9 Discussion . . . . . . . . . . . . . . . . . . 178

6 Preparing the Virtual Ocean for the LaMSS simulation suite 181

6.1 Introduction to the LAMSS simulation suite . . . . . . . . . . . . . . . . 181

6.2 Task Force Ocean \& the Irminger Sea . . . . . . . . . . . . . . . . . . 183

6.3 Software Handshaking with Environmental Inputs ． . . . . . . . . . . . 185

6.4 Integrating NOAA Bathymetric Data . . . . . . . . . . . . . . . . . . 186

6.5 Creating Environmental EOFs . . . . . . . . . . . . . . . . . . 187

6.6 Manually identifying benchmark cases . . . . . . . . . . . . . . . . . . . . 194

6.7 K-means clustering benchmark cases . . . . . . . . . . . . . . . . . . 195

6.8 Bootstrapping Synthetic Benchmark Cases ． . . . . . . . . . . . . . . . . . . 198

6.9 Discussion . . . . . . . . . . . . . . . . . . . 200

7 Conclusion 201

7.1 Summary of Contributions . . . . . . . . . . . . . . . . . . . . 202

7.2 Future work . . . . . . . . . . . . . . . . . . . 203

7.3 Closing comments . . . . . . . . . . . . . . . . . . . . 210

$\begin{array}{ll}\text { Appendix } & 213\end{array}$

A. GPS drift during ICEX20 . . . . . . . . . . . . . . . . . . . . . 214

B. Through the sensor characterization of the Beaufort Lens from A/V Macrura . . . 215

C. Running an EOF mission in LAMSS . . . . . . . . . . . . . . . . . . . . 216

$\begin{array}{ll}\text { Bibliography } & 223\end{array}$ 


\section{List OF Figures}

1.1 Historical and observed Arctic sound speed profiles and ray propagations for the

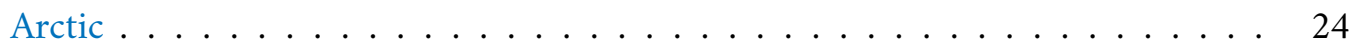

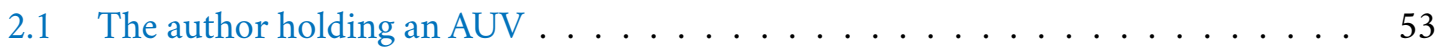

2.2 Spatio-temporal scales of ocean features . . . . . . . . . . . . . . 57

3.1 WHOI Ice Tethered Profiler Overview $\ldots \ldots \ldots$. . . . . . . . . . . 65

3.2 Map of ITP location density . . . . . . . . . . . . . . . . . . . . . . 67

3.3 Duct strength calculation . . . . . . . . . . . . . . . . . 74

3.4 Duct strength statistics $\ldots \ldots \ldots \ldots \ldots \ldots \ldots \ldots \ldots$

3.5 A heat map of sound speed profile distribution by duct strength . . . . . . . . 76

3.6 Temporal trends for duct strength $\ldots \ldots \ldots \ldots \ldots$. . . . . . . . 80

3.7 Temporal trends for temperature difference inside the duct . . . . . . . . . . . . 81

3.8 Temporal trends for knee depth . . . . . . . . . . . . . . . . . . . . 82

3.9 Probability distribution of duct top and bottom depths . . . . . . . . . . . 83

3.10 Map of median duct strength across the Beaufort Gyre . . . . . . . . . . . . . 85

3.11 Mapping knee height versus surface layer depth . . . . . . . . . . . . 88

3.12 Representative salinity, temperature, and sound speed profiles for ICEX16 _ . . 90

4.1 Visual diagram of the Virtual Ocean approach . . . . . . . . . . . . . . . 105 
4.2 Diagram of the interactive TDA algorithm . . . . . . . . . . . . . . 107

4.3 Northern Hemisphere daily sea ice extent . . . . . . . . . . . . . . . . . 111

4.4 Base sound speed values for different tuned training sets . . . . . . . . . . . . . 112

4.5 EOF shapes and significance . . . . . . . . . . . . . . . . 113

4.6 Kernel Density Estimates for each EOF . . . . . . . . . . . . . . . . . 114

4.7 Choosing an EOF set for the mission . . . . . . . . . . . . . 115

4.8 ICEX20 CTDs with chosen sound speed estimates compared to HYCOM, ITP . 119

4.9 Chosen weights against a priori distributions . . . . . . . . . . . . . . . 121

4.10 EOF priority throughout ICEX20 . . . . . . . . . . . . . . . . 122

4.11 Comparing acoustic and environmental penalties . . . . . . . . . . . . . 124

4.12 TDA: all sound speed combinations _ . . . . . . . . . . . . . . 127

4.13 TDA: weight distribution compared to training set $\ldots \ldots \ldots \ldots \ldots$

4.14 TDA: absolute ray shift . . . . . . . . . . . . . . . . . . . . . 129

4.15 TDA: relative ray shift $\ldots \ldots \ldots \ldots \ldots \ldots \ldots \ldots$

5.1 An overview of the modem experiment by source and receiver location . . . . . 141

5.2 A timeline of source and receiver depth throughout the modem experiment . . . 143

5.3 Sound speed estimates used for in situ and post-processing analysis . . . . . . . . 144

5.4 Ice floe drift during ICEX20 . . . . . . . . . . . . . . . . . . . . . 145

5.5 Eigenrays for the three sound speed environments for source depth $=20 \mathrm{~m} \ldots \ldots 147$

5.6 Eigenrays for the three sound speed environments for source depth $=30 \mathrm{~m} \ldots \ldots 149$

5.7 Eigenrays for the three sound speed environments for source depth $=90 \mathrm{~m} \ldots \ldots 150$

5.8 Comparing the naive and in situ simulated group velocity over range . . . . . . 153

5.9 In situ range error by source-receiver depth pairings . . . . . . . . . . . . . . 159

5.10 Cross-map of range estimation performance for the baseline sound speed profile . 161

5.11 Cross-map of range estimation performance for the weighted sound speed profile 162 
5.12 Cross-map of post-processing range estimation performance for all three sound speed environments . . . . . . . . . . . . . . . . . . . 165

5.13 Group velocity estimates in post-processing approach . . . . . . . . . . . . 168

5.14 Range error in post-processing approach . . . . . . . . . . . . . . 170

5.15 Histogram of post-processed range error . . . . . . . . . . . . . . . 171

5.16 Histogram of post-processed range error by number of bounces . . . . . . . . 173

5.17 Examination of GPS and modem drift . . . . . . . . . . . . . . 178

6.1 Bathymetric map of the Irminger Sea . . . . . . . . . . . . . . . . . . 187

6.2 Monthly sound speed profiles in Irminger Box $3 \ldots \ldots$. . . . . . . . . 189

6.3 Optional smoothing parameter for EOF shapes . . . . . . . . . . . . . . 191

6.4 Resultant EOF shapes . . . . . . . . . . . . . . . . . . . . . . . . . . . 192

6.5 KDEs saved into the EOF set . . . . . . . . . . . . . . . . . . . 193

6.6 Manually chosen benchmark cases . . . . . . . . . . . . . . . . . . 195

6.7 K-means clustering of sound speed profiles . . . . . . . . . . . . . . 196

6.8 Spatial projection of clustered sound speed profiles . . . . . . . . . . . . . 197

6.9 Bootstrapping SSPs from an EOF set . . . . . . . . . . . . . . . . . . . 199 


\section{List OF TABLES}

4.1 Environmental-dominated TDAs in Tactical Environmental Support System . . . 101

4.2 Default ray tracing parameters for TDA . . . . . . . . . . . . . . . 113

4.3 Ranking of sound speed estimates . . . . . . . . . . . . . . . . 117

5.1 Range estimation error for in situ events . . . . . . . . . . . . . . . . . . . 157

5.2 Comparison of range estimation algorithms for real-time group velocity predicted

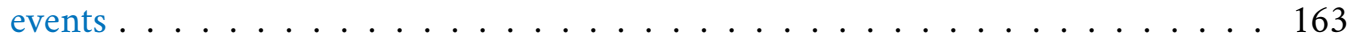

5.3 Comparison of post-processing range estimation algorithms across all events . . . 166

5.4 Further look at algorithm differences for one beacon to beacon pairing . . . . . 167

6.1 Weights for clustered benchmark cases . . . . . . . . . . . . . . . . . . 197 


\section{Acronyms}

\begin{tabular}{ll} 
AUV & Autonomous Underwater Vehicle \\
CSAC & Chip Scale Atomic Clock \\
CTD & Conductivity, Temperature, and Depth \\
EOF & Empirical Orthogonal Function \\
GNSS & Global Navigation Satellite System \\
GPS & Global Positioning System \\
HYCOM & Hybrid Coordinate Ocean Model \\
ICEX16/ICEX20 & Ice Exercise 2016/2020 \\
ICNN & Integrated Communication and Navigation Network \\
ITP & Ice Tethered Profiler \\
KDE & Kernel Density Estimate \\
MBC/NBC & Minimal/Nearest Bounce Criteria \\
OWTT & One-way travel time \\
PCA & Principal Component Analysis \\
PSW & Pacific Summer Water \\
PWW & Pacific Winter Water \\
SSP & Sound Speed Profile \\
TDA & Pactical Decision Aid \\
\hline
\end{tabular}




\title{
1 Introduction
}

\author{
पृथिवी वायुर् आकाशम् आपो ज्योतिः च राघवः | \\ स्वभावे सौम्य तिष्ठन्ति शाश्वतम् मार्गम् आशरिताः ॥ \\ वाल्मीकि, रामायणम, युद्ध कन्धा २२-२५
}

O beloved Rama! Earth, wind, water, and light remain fixed in their own nature, resorting to their eternal path

Valmiki, Ramayana, Book of War, Chapter 22 Verse 25

More than twenty-five hundred years ago, Valmiki, a revered poet and sage, scribed the epic Ramayana. In one scene, Rama, the titular exiled prince, attempts to cross the ocean between the southern tip of India and Sri Lanka to wage battle against Ravana, a demon king, and rescue his wife, Sita, whom Ravana abducted. Frustrated that the ocean will not part ways for him to continue his journey, Rama shoots arrows at the various sea monsters, and then brandishes an enchanted arrow to dry up the ocean completely. The ocean, at first aloof to the demands of the prince, recognizes the gravity of the situation and explains its actions-it could not part ways for Rama even if it wanted to. But to solve this impasse, the ocean reminds Rama that there just so happens to be a skilled engineer in his forest-dwelling men/monkey army, and permits them to build a bridge across itself. ${ }^{1}$

\footnotetext{
${ }^{1}$ Amazingly, there's geological evidence for this land connection, now referred to as Rama Setu or Adam's Bridge
} 
I grew up with this story, which distills some longstanding truths about the spirit of this dissertation. The ocean obeys eternal, physically-driven laws and it is beyond mortal capacity to change them. When we seek to unerstand them and navigate its waters, we hope we have the right tools, expertise, and a little bit of luck.

\subsection{Problem statement}

One popular tool to explore the ocean is the autonomous underwater vehicle (AUV). Lightweight, mobile, and relatively inexpensive, these platforms are becoming increasingly capable with smaller payload sensors, stronger computing power, and smarter artificial intelligence. ${ }^{2}$ But a major hurdle in considering AUVs as reliable, standard tools for oceanographic research is navigation uncertainty. ${ }^{3}$ While land- and air-based robots utilize satellite-based global positioning system (GPS) with stunning accuracy and precision throughout the duration of their missions, AUVs can only stall on the surface to receive a position update; they cannot access GPS while underwater. In an ice-covered environment (and other GPS-denied situations), even this foolproof method of re-positioning is inaccessible.

A far more ubiquitous tool in oceanography is acoustic telemetry, which informs a wide array of sensing, communication, and navigation modalities. For underwater robotics, communication and navigation are inherently linked because of the dominance of acoustics as the information-dense modality. Different forms of acoustically-driven underwater positioning systems, spanning a range of frequencies, operational scales, and hardware devices, provide some analog to GPS-instead of satellites orbiting the Earth, beacons are fixed throughout an ocean volume. Acoustic underwater positioning systems convert the recorded travel time between two beacons into the distance between them. In real-time operations, this conversion

\footnotetext{
${ }^{2}$ Bellingham et al., "Robotics in Remote and Hostile Environments".

${ }^{3}$ There is little comfort that, when deployed, you cannot physically see the vehicle
} 
has always been deterministic, oversimplifying any environmental variability and dynamics, and by extension, the non-linearity between the environment and acoustic propagation. Results from this work are the first field deployment where an in situ acoustic ray tracing method accounts for arrival structure in estimating range.

The titular idea in this thesis, the Virtual Ocean, provides a working solution for an updatable, depth-dependent sound speed estimate on a submerged asset. This sound speed estimate informs embedded acoustic modeling for accurate navigation and communication, a key advancement for AUVs to be adapted as standard tools for short- and long-term monitoring in oceanographic studies and ocean observatories. This work is motivated by challenges from ice-covered AUV operations in March 2016, when a prominent sound speed lens significantly disrupted communication and navigation. ${ }^{4}$ The success of this approach is ultimately demonstrated by field results from AUV operations in March 2020. I will give a brief overview of why the Arctic is a region of significant overlapping interests before discussing the operational challenges faced in March 2016.

\subsection{A new, Blue Arctic}

The Arctic, a generally cold, cold place, has been warming rapidly over the past three decades. The Arctic is one of the most sensitive regions to anthropogenic climate change, in part due to the feedback mechanism of water absorbing a greater proportion of the sun's heat than ice does. ${ }^{5}$ Permafrost melt threatens existing Arctic social, military, and commercial infrastructure $^{6}$ while also destabilizing the region's role in the global carbon cycle, from carbon sink to

\footnotetext{
${ }^{4}$ Schmidt et al., "Acoustic Communication and Navigation in the New Arctic - A Model Case for Environmental Adaptation".

${ }^{5}$ Chapin et al., "Role of Land-Surface Changes in Arctic Summer Warming".

${ }^{6}$ Khurshudyan et al., An oil spill in Russia's Arctic exposes problems in Moscow's big plans for the Far North.
} 
carbon source. ${ }^{7}$ Sea ice melt, driven by warmer ocean temperatures, ${ }^{8}$ creates a more accessible Arctic for human activity and mining natural resources but also disrupts local ecosystems and regional food webs. Sea level rise, driven by meltwater from polar ice sheets, directly threaten the livelihoods of nearly $40 \%$ of the world's population that live in a coastal zone. ${ }^{9}$ And ocean acidification, accelerated in colder water due to higher solubility of gaseous carbon dioxide, ${ }^{10}$ corrodes the minerals vital to shell-building creatures and destabilizes commercial and subsistence fisheries. ${ }^{11}$ When we turn to data, the previous year, 2020, recorded the sea ice minimum as the $2^{\text {nd }}$ lowest on satellite record (since 1978) and the near-surface air temperature as the $2^{\text {nd }}$ warmest year since at least $1900 .{ }^{12}$

Indeed, in August 2017, the National Science Foundation (NSF) listed "Navigating the New Arctic" as one of its Ten Big Ideas. ${ }^{13}$ It acknowledged that "current Arctic observations are sparse and inadequate for enabling discovery or simulation of the processes underlying Arctic system change or to assess their environmental and economic impacts on the broader Earth system" and called for developments in "observing network[s] mobile and fixed platforms ... to document and understand the Arctic's rapid biological, physical, chemical, and social changes." This call for proposals reaches a wide set of disciplines, spanning and connecting climate forecasting, the built and natural environment, preservation of Indigenous communities and knowledge, and synthesizing big environmental data.

The interest in the changing Arctic couples climate security with national security. In January 2021, the Department of the Navy released a strategic blueprint ${ }^{14}$ on A Blue Arctic-one

\footnotetext{
${ }^{7}$ MacDougall et al., "Significant contribution to climate warming from the permafrost carbon feedback".

${ }^{8}$ Timmermans et al., "Warming of the interior Arctic Ocean linked to sea ice losses at the basin margins".

${ }^{9}$ Lustgarten, “The Great Climate Migration Has Begun”.

${ }^{10}$ Cross et al., "Formation and transport of corrosive water in the Pacific Arctic region".

${ }^{11}$ Mathis et al., "Ocean acidification risk assessment for Alaska's fishery sector".

${ }^{12}$ NOAA Arctic Report Card 2020 [link]

${ }^{13}$ NSF Big 10 Ideas: Navigating the New Arctic [link]

${ }^{14}$ Department of the Navy: A Strategic Blueprint for a Blue Arctic [link]
} 
without ice. The report declares the United States as a maritime and Arctic nation whose "security, prosperity, and vital interests in the Arctic are increasingly linked to those of other nations in and out of the region." As melting sea ice creates navigable (and more polluted) seas, maritime routes linking Asia, Europe, and North America have the potential to connect nearly $75 \%$ of the world's population. These changing conditions heighten the potential for ecosystem collapse, tourism, accidents at sea, and military activity.

One cannot overestimate the importance of high quality data and monitoring systems when considering the environmental volatility and the intersecting social, political, and economic repercussions. Current data streams for polar observations largely rely on satellites ${ }^{15}$ which, while incredibly advantageous for high temporal and spatial resolution, provide little information about the water column. Autonomous vehicles are one tool-deployed individually, in a fleet, or in heterogeneous networks-that will be essential for the next generation of smart data collection in polar environments.

\subsection{UNDERWATER NAVIGATION AND COMMUNICATION IN THE}

\section{New Arctic}

Underwater vehicle deployments during the Ice Exercise in 2016 (ICEX16) demonstrate why acoustic communication is co-evolutionary with vehicle autonomy and navigation. ${ }^{16}$ Our lab, the MIT Laboratory for Autonomous Marine Sensing Systems, deployed an AUV with a towed hydrophone array below the ice cover to assess the ambient noise environment. Successful operations of the AUV depended on navigation updates from a tracking solution, communicated to the vehicle to fuse with onboard inertial navigation.

\footnotetext{
${ }^{15}$ Naranjo, The modern sea ice satellite record turns 40.

${ }^{16}$ Schmidt et al., "Acoustic Communication and Navigation in the New Arctic - A Model Case for Environmental Adaptation".
} 

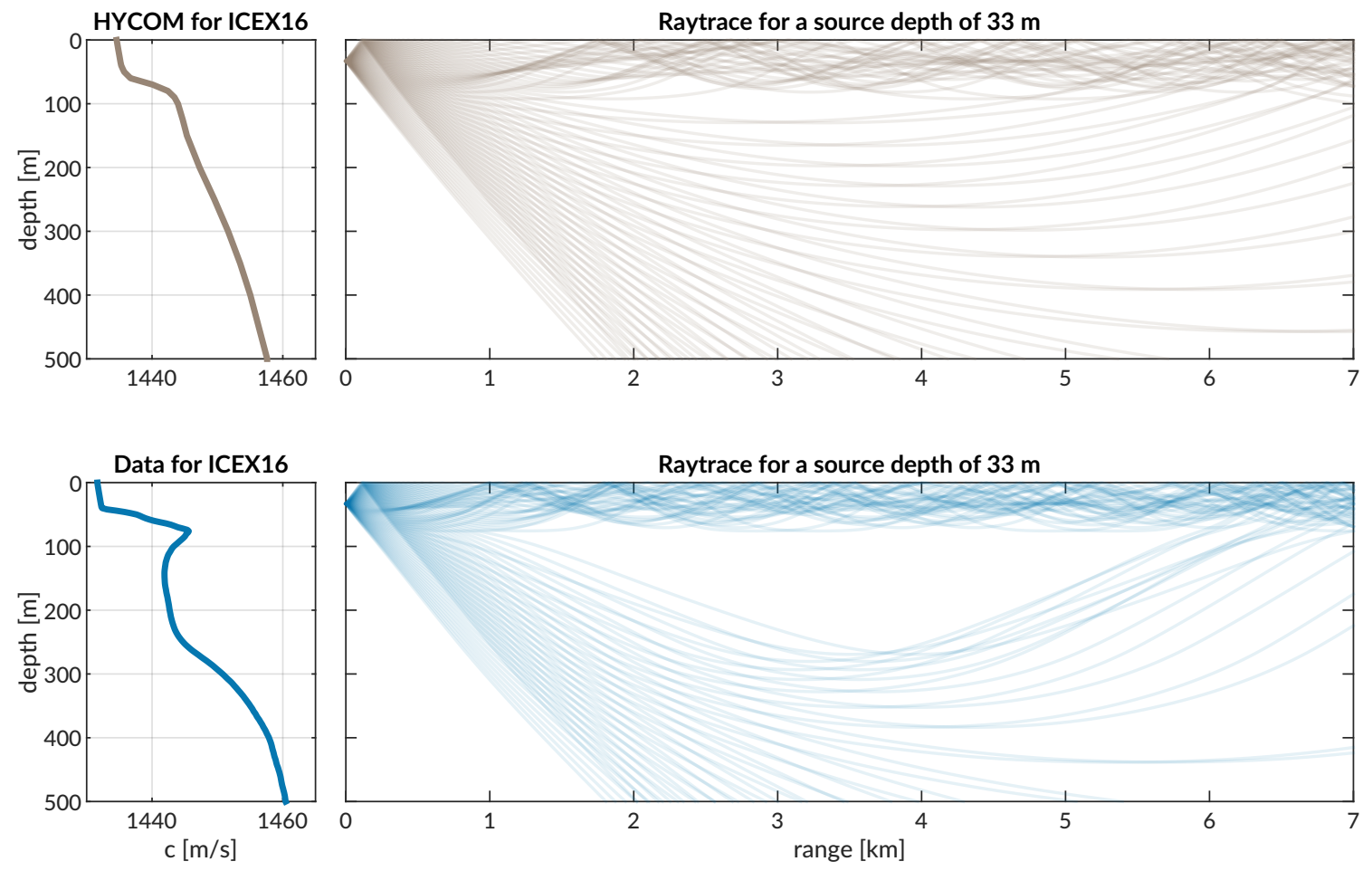

Figure 1.1: A comparison of the historical and observed Arctic sound speed profiles and ray propagations. The top is the classical Arctic profile. The bottom is the observed sound speed profile during ICEX16. The rays here are launched within a cone of $\pm 17^{\circ}$ to propagate without bottom interaction.

The changes in the environment, highlighted in figure 1.1, severely deteriorated the tracking performance compared to historical conditions in the Arctic. This sound speed feature, often called the Beaufort Lens, ${ }^{17}$ is a neutrally buoyant layer of warm Pacific water at depth of 50-70 m, creating a local maximum in the sound speed profile (SSP). The historical SSP in the Arctic is monotonically increasing, such that the acoustic propagation is fairly straightforward, with all rays bending upward and interacting off the ice cover. The Beaufort Lens creates an upper and lower duct, where upper duct degrades signal coherence due to intensified ice interaction, and the lower duct effectively traps sound for long range propagation. This change, with obvious implications for long range acoustic propagation, is equally important for shorter ranges as well, where a shadow zone is created by a drastic change in the reliable

\footnotetext{
${ }^{17}$ Poulsen et al., "Acoustic noise properties in the rapidly changing Arctic Ocean".
} 
acoustic path. The changing acoustic environment necessitated environmentally adaptive autonomy for robust navigation and communication for a vehicle deployment in March 2020.

\subsection{Virtual Ocean approach}

This thesis, in preparation for the Ice Exercise in March 2020 (ICEX20), presents an embedded Virtual Ocean paradigm to anticipate the core challenges faced during ICEX16.

First, in pre-deployment, basis function representations of the environment are created and loaded onto the vehicle. This is a statistical compression of the sound speed variability that is easily parameterized with a handful of weights. The sound speed training set is derived from the available oceanographic models and observational data.

Next, during operations but before a vehicle goes underway, an external CTD (conductivity, temperature, and depth) cast is taken to get a local observation of the sound speed. Through an interactive and graphical decision making framework, a decision-maker verifies the observation, and chooses an initial basis set and weights to recreate the sound speed. The latter is sent to the vehicle via an acoustic message as it begins its mission.

Throughout the mission, given another CTD cast, a topside decision-maker can solve for new weights and send them to the vehicle, updating the sound speed estimate that drives the vehicle's environmentally adaptive communication and navigation behaviors. Importantly, the selection of weights is driven by environmental and acoustic penalties.

More broadly speaking, while many underwater acoustic navigation solutions use a deterministic sound speed, an updatable depth-dependent SSP is necessary for acoustically complex environments, regardless of operational spatial scales. This system was successfully demonstrated in ICEX20, with a multitude of CTD casts, messages sent to submerged assets, and at the end of the experiment, successful recovery of the AUV. 


\subsection{Thesis ORgANIZATION \& CONTRIBUTIONS}

This dissertation is divided into seven chapters. This first chapter briefly frames the problem statement and articulates the overall research direction for the remaining chapters.

Chapter 2 provides a background and literature review that contextualizes the new research presented in this dissertation. It begins with a review on Global Navigation Satellite System (GNSS) and establishes the interdependent nature of acoustic communication and underwater navigation systems. A historical lens on measuring sound speed and ray tracing contextualizes the applied nature of ocean acoustics. Then, the chapter introduces the rapid development of autonomy in AUVs as is relevant to embedded acoustic sensing. Lastly, there is a discussion on the spatio-temporal scales of ocean features and environmentally adaptive autonomy. A summary at the end of the chapter connects the dots on how each section relates to the contributions of this thesis.

Chapter 3 explores the WHOI Ice Tethered Profiler dataset to better understand the sound speed variability of the Beaufort Lens. The key contributions are:

- creating an automated pipeline to analyze ITP data in near real-time for vehicle operations in the Arctic

- quantifying the strength and variability of the Beaufort Lens since 2006 and corroborating its widespread but discontinuous nature, which previously has only been suggested by acoustic fluctuations and described by model efforts ${ }^{18}$

Chapter 4 presents the development of the interactive and graphical decision making framework to distill the most up-to-date sound speed information and convey the joint environ-

\footnotetext{
${ }^{18}$ Duda et al., "Long-range sound propagation in the Canada Basin".
} 
mental realism and acoustic utility for parameterized sound speed estimates. An empirical orthogonal function analysis is chosen to create the basis functions, and the Tactical Decision Aids (TDAs) simplify the non-linear environmental and acoustic relationship for a human-inthe-loop decision maker. The key contributions are:

- designing an interactive TDA framework to distill sound speed estimates into a handful of weights, and to evaluate those sound speed estimates with parameterized algorithms for environmental and acoustic penalties

- deploying this TDA system during ICEX20 to capture a temporally varying sound speed duct

- transmitting weights via acoustic communication to update the internal sound speed estimate on submerged assets

Chapter 5 validates the need for an internal sound speed estimate as well as an embedded, real-time, stochastic group velocity prediction for accurate range estimation by examining acoustic events between GPS-connected modem buoys during ICEX20. The key contributions are:

- seeding real-time, stochastic group velocity estimates as a function of source depth and sound speed estimates to achieve GPS-like accuracy; this is, to my knowledge, the first time this has been done in a real-time experiment

- improving the suggested stochastic group velocity estimation with a nearest multi-path criteria to achieve range accuracy that rivals GPS accuracy and surpasses GPS precision

Chapter 6 describes the software infrastructure of the Virtual Ocean, in the context of future work studying the Irminger Sea. The key contribution is the framework for any acoustics, oceanographer, or marine robotics researcher interested in using the Virtual Ocean to 
study embedded acoustic processing in a high-fidelity, robust simulation environment, for any space-time region of interest.

Chapter 7 contains a summary of contributions, a discussion of future work, and closing comments. 


\section{Background}

To sense this world of waters known to the creatures of the sea we must shed our human perceptions of length and breadth and time and place, and enter vicariously into a universe of all-pervading water. For to the sea's children nothing is so important as the fluidity of their world. It is water that they breathe; water that brings them food; water through which they see, by filtered sunshine from which first the red rays, then the greens, and finally the purples have been strained; water through which they sense vibrations equivalent to sound. And indeed it is nothing more or less than sea water, in all its varying conditions of temperature, saltiness, and pressure, that forms the invisible barriers that confine each marine type within a special zone of life...

Rachel Carson, Undersea (1937)

The name of our planet is, of course, a misnomer. Seventy percent of Earth is covered by water, and many mechanisms that we live and innovate by on and above the surface play differently as we plunge below the sea. Light waves, which touch everything the eye can see, hold little sway beyond the first couple hundred meters in the ocean. Radio waves, which connect Earth to intrepid robots on Mars or locate a cell phone relative to a handful of satellites, break down in the first few meters underwater. But sound waves, which many terrestrial creatures use to communicate over short distances, travel five times faster and easily tens of thousands times farther underwater than through air. As Carson eludes, sound, being sensitive to the conditions that make water itself-temperature, salinity, and pressure-is the natural way to sense the world of waters. 
This chapter is an overview of many interconnected ideas that are fundamental to this work. The advanced reader may be interested in section 2.7, the summary at the end of the chapter, that distills how each concept builds off the previous and contextualizes it for the work in this dissertation.

\subsection{Global Navigation Satellite System}

The overarching motivation for this thesis is accurate navigation for underwater vehicles in under-ice or GPS-denied environments. But to best understand the challenges in underwater navigation, we first introduce its precursor, terrestrial- and air-based navigation.

The Global Navigation Satellite System (GNSS) drives localization, tracking, navigation, mapping, and timing, which are now everyday applications. What we commonly refer to as GPS, the Global Positioning System, is the US owned system, operated by the US Air Force from Shriever Air Force Base, Colorado. The first GPS system, Navigation System with Timing and Ranging (NAV-STAR), was launched in 1978; the 24-satellite system was fully operational in $1995 .^{1}$ There are three other GNSS systems-Russian (GLONASS), Chinese (BeiDou), and European (Galileo)—of those, only Galileo is a purely civilian system. ${ }^{2}$

The physics and technology behind GNSS, while incredibly advanced, is intuitive and easy to understand. GPS satellites orbit around 20,000 $\mathrm{km}$ above the Earth, where their position is tracked and communicated by ground stations using radar, signal doppler, and laser reflectors. The satellites continuously broadcast their location with precise timing and location information via electromagnetic waves, generally in the microwave band. These signals travel at the speed of light, $c$, to any receiver, say, a cell phone, or a GPS unit in a car. The time of flight, $t$, is

\footnotetext{
${ }^{1}$ I lived through the transition from no longer printing out MapQuest directions to getting mad at the GPS for "misdirecting" us to away soccer games.

${ }^{2}$ Van Uffelen, "Global Positioning Systems: Over Land and Under Sea".
} 
how long the signal takes to reach the receiver from any satellite. Given one time of flight, the receiver could be anywhere on a sphere of some radius, $r$, by the simple relationship between distance, speed, and time: ${ }^{3}$

$$
r=c \cdot t
$$

Given at least four time of flights from four distinct, well-placed satellites, the receiver is at the intersection of those spheres, which can be a single point in three-dimensional space. Because the speed of light is large, $3 \cdot 10^{8} \mathrm{~m} / \mathrm{s}$, nanosecond resolution on the satellites is necessary to achieve positioning error on the order of meters. This timing is achieved by an atomic clock, which is actually corrected for drift twice a day using an even higher precision reference atomic clock on land.

For fieldwork-in water, land, or air-GNSS is standard procedure, providing geolocation for any multitude of observations. The exact metadata becomes crucial when reconstructing spatio-temporal fields, as is often the case in robotic applications. And even though satellite navigation was designed with submarines in mind, it does not work when vehicles are beneath the sea surface. The following sections will more thoroughly examine why equation 2.1 does not easily transfer to sound waves propagating through the water column.

\subsection{UNDERWATER ACOUSTIC COMMUNICATION}

The undersea medium poses significant challenges compared to wireless communication. Electromagnetic signals, which undergird GNSS, experience high rates of absorption in sea water. Optical signals are also rapidly absorbed in sea water, scattered by suspended particles, and

\footnotetext{
${ }^{3}$ GNSS is slightly more complicated than this, but this is an extremely accurate first order approximation
} 
drowned out by ambient light in the upper water column. Acoustic signals are the primary but by no means ideal form of wireless underwater communication. The physics behind their propagation introduces several important limiting factors compared to that of electromagnetic waves through the atmosphere. ${ }^{4}$

1. Spreading loss - the expansion of a fixed amount of energy over a larger and larger surface area. This changes from spherical to cylindrical from short to long ranges.

2. Absorption loss-particle motion transferred into heat via viscous drag and via chemical relaxation. Absorption has three contributions, from boric acid (more significant at lower frequencies), magnesium sulphate (more significant at higher frequencies), and pure water. ${ }^{5}$ Overall the absorption loss is strongly frequency dependent and increases with frequency.

3. Surface scattering-when a rough sea surface (or bottom) introduces a spreading in the delay of each surface bounce path, resulting in high intensity and rapidly fluctuating arrivals.

4. Ambient noise-natural sources of noise in the ocean at frequencies of interest. These include breaking waves, bubbles, rain, and biological sources like whales, dolphins, and snapping shrimp.

5. Latency-the slow speed of propagation, $1500 \mathrm{~m} / \mathrm{s}$, compared to $3 \times 10^{8} \mathrm{~m} / \mathrm{s}$ for light in a vacuum. The channel coherence time dwarfs the travel time for signals that travel greater than $100 \mathrm{~m}$.

\footnotetext{
${ }^{4}$ Kilfoyle et al., "The state of the art in underwater acoustic telemetry"; Preisig, "Acoustic propagation considerations for underwater acoustic communications network development"; Schneider, "Advances in Integrating Autonomy with Acoustic Communications for Intelligent Networks of Marine Robots"; Stojanovic et al., "Underwater acoustic communication channels".

${ }^{5}$ Ainslie et al., "A simplified formula for viscous and chemical absorption in sea water".
} 
6. Doppler spreads-the relatively slow speed of propagation results in shifts of recognized signals resulting from the propagation path fluctuations due to platform motion or scattering.

7. Multipath-multiple delayed copies of a signal can reach the receiver primarily due to significant refraction in a stratified ocean environment and/or surface (or bottom) bounces.

8. Low bandwidth - primarily due to absorption, the ocean is a lowpass filter for acoustic waves. Packet information is constrained to $10^{2} \mathrm{bits} / \mathrm{second}$, compared to $10^{4} \mathrm{bits} / \mathrm{sec}-$ ond for Earth-Mars transmissions and $10^{8}$ bits/second for wireless Internet. Sending higher bandwidths of data to match terrestrial and space capabilities requires an unreasonable amount of power, potentially harming or disrupting marine life.

9. Intermittency - the ocean is a spatially and temporally varying medium. The path sound takes between a source and a receiver may change, or no longer exist, with some cycles of variability on the order of minutes and others on the order of seasons or years.

Of particular concern for this thesis are the latter three-multipath, low bandwidth, and intermittency—given their implications for signal arrival structure, minimal message content, and message success. The next two sections will give a brief overview of how sound speed is a function of temperature and salinity, and then how ray tracing is sensitive to those physical parameters.

\subsubsection{Calculating sound SPeEd}

We can trace back ocean acoustics research to something we now take for granted-measuring the speed of sound in water. We understand that Italian renaissance artist Leonardo da Vinci, 
in 1490 , was the first to document some intuition about underwater acoustics. ${ }^{6}$ He noticed that if you placed a long tube in the water to your ear, you could hear ships at a great distance, inferring that the speed of sound in water was much greater than the speed of sound in air.

In 1687, English mathematician Isaac Newton introduced the first mathematical treatment of sound, ascertaining that the speed of sound could be calculated by the square root of the pressure divided by the medium's density. In 1816, French polymath Pierre-Simon LaPlace realized that Newton's calculation neglected the influence of heat, and introduced the elastic bulk modulus, which is how much a material will compress under a given amount of external pressure.

Building on these ideas, Swiss physicist Daniel Colladon and French mathematician Charles Sturm, in 1826, were interested in measuring water's compressibility and produced the first reliable measurement of sound speed in water. They inferred that the speed of light was infinitely faster than the speed of sound in water, and measured the elapsed time between a flash of light and the sound of a ship's bell heard using a submerged listening horn. They measured the speed of sound to be $1435 \mathrm{~m} / \mathrm{s}$ over a $17 \mathrm{~km}$ distance, which is correct to about $0.02 \%$ of currently accepted values at that temperature, salinity, and pressure.

This initial experiment could only realistically find a static value for the speed of sound in water, in the same way that we have a nominal speed of light; it of course varies with depth, i.e. compressability, creating a sound speed profile. $^{7}$ An active field of research in the 1960 s-80s was finding and optimizing empirical methods to measure vertical sound speed structure.

In 1960, Wilson from the U.S. Naval Ordnance Laboratory, analyzed a total of 581 sound speeds measured at fifteen temperatures, eight pressures, and five salinities in ranges that described $99.5 \%$ of all sea water ( -3 to $30 \mathrm{C}, 1.033$ to $1000 \mathrm{~kg} / \mathrm{cm}^{2}$, and 33 to $\left.37 \mathrm{PSU}\right){ }^{8}$ The

\footnotetext{
${ }^{6}$ Urick, Principles of Underwater Sound, third edition.

${ }^{7}$ Previous literature calls it the sound velocity profile, but there is no direction implied

${ }^{8}$ Wilson, "Speed of Sound in Sea Water as a Function of Temperature, Pressure, and Salinity".
} 
sound speeds were measured using an ultrasonic interferometer, a simple but rather brilliant device. First, a vibrating quartz crystal produces ultrasonic waves via the piezoelectric method that radiate into a small, insulated quantity of the experimental medium. A movable metallic plate sits parallel to the quartz one, which reflects the waves back. If the separation between the plates is exactly an integer multiple of half-wavelengths of sound, standing waves are produced, and acoustic resonance occurs. The resonant waves are a maximum in amplitude and cause a corresponding maximum in the anode current of a piezoelectric generator. Modulating the lower plate to find the next standing wave finds $d=\lambda / 2$. From there, the sound speed is determined to be $c=\lambda f$. Thus, Wilson ran several tiny and meticulous experiments with controlled seawater samples, and then used a 704 IBM computer to solve for an equation for sound speed using the method of least squares.

In 1972, Del Grosso and Mader replicated the ultrasonic interferometer method in pure water. $^{9}$ With a total of 148 observations, they fit a fifth order in temperature sound speed equation with a standard deviation of $0.0028 \mathrm{~m} / \mathrm{s}$, where the valid temperature range was between 0.001 and $95.126 \mathrm{C}$. The paper itself is as many tables as it is pages. A follow up paper, in 1974, introduced a new equation for the speed of sound in pure water and sea water with realistic combinations of salinity, temperature, and pressure, which is one of a few that are widely used today. ${ }^{10}$ The equation, nominally referred to as NRL II, addressed scenarios where "the sound speed differences are of greater importance than the actual sound speeds". This was a prescient finding considering the temporal resolution necessary for underwater navigation.

Three years later, Chen and Millero squared a confusing result-the 1-atm sound speeds of Del Grosso were more reliable than Wilson's, but the high-pressure results of Wilson were

\footnotetext{
${ }^{9}$ Del Grosso et al., "Speed of Sound in Pure Water".

${ }^{10}$ Del Grosso, "New equation for the speed of sound in natural waters (with comparisons to other equations)".
} 
more reliable than Del Grosso's. ${ }^{11}$ Their equation explicitly accounts for the effect of pressure on the relative speeds on sound and is valid for sea water and pure water. With that correction, their equation agreed with the low pressure values of Del Grosso and the high pressure values of Wilson. The Chen and Millero equation is another standard in ocean acoustics and forms the basis for the international standard algorithm, often called the UNESCO equation.

In 1981, Mackenzie introduced a nine-term equation for sound speed in the ocean that inputs temperature (-2 to $30 \mathrm{C}$ ), salinity (25 to $40 \mathrm{psu}$ ), and depth (0 to $8000 \mathrm{~m})$, not pressure. ${ }^{12}$ His work was driven by the then recent availability of programmable handheld calculators. The MacKenzie equation yielded a standard error of $0.070 \mathrm{~m} / \mathrm{s}$ for the 14,315 points that made the "training set".

$$
\begin{array}{r}
c=1448.96+4.591 T-5.304 \cdot 10^{-2} T^{2}+2.374 \cdot 10^{-4} T^{3}+ \\
1.340(S-35)+1.630 \cdot 10^{-} 2+1.675 \cdot 10^{-7} D^{2}- \\
1.025 \cdot 10^{-2} T(S-35)-7.139 \cdot 10^{-13} T D^{3}
\end{array}
$$

This thesis predominantly uses the Mackenzie formulation for its simplicity, extended temperature bounds, and accuracy for short-range acoustics. ${ }^{13}$ It should be noted, in an unfortunate clerical error, the salinity domain is misstated in the abstract compared to the manuscript itself. This led to a small crisis in the days before ICEX20, when salinity values less than 30 caused some software processes to flag an error. Later efforts, using long-range acoustic transmissions with extensive environmental measurements, suggested that Del Grosso performed better than Chen and Millero. ${ }^{14}$

\footnotetext{
${ }^{11}$ Chen et al., "Speed of sound in seawater at high pressures".

${ }^{12}$ Mackenzie, "Nine-term equation for sound speed in the oceans".

${ }^{13}$ Jensen et al., Computational Ocean Acoustics.

${ }^{14}$ Dushaw et al., "On equations for the speed of sound in seawater".
} 
Overall, temperature is the dominant variable affecting sound speed in the upper few hundred meters of the ocean. With increasing depth, temperature and salinity have less variability, and pressure dominates the sound speed calculation. Salinity is generally less important except for specific environments where its variance is more drastic than temperature (say, polar regions, or an estuary). The tremendous amount of work that went into measuring sound speed accurately enables high confidence in minor travel time differences; a depth-dependent sound speed profile (SSP) is essential to understanding how sound propagates in the ocean.

\subsubsection{Ocean acoustic Ray tracing}

Ray tracing is an elegant extension of the Law of Refraction, which was discovered (and generally taught) with respect to optics. This law, commonly attributed to Snell (1621) or Descarte (1637), was first written down in 984 by Ibin Sahl, a Persian mathematician and physicist of the Islamic Golden Age. It states that, in two media, the ratio of the sines of the angles of incidence and refraction is equivalent to the ratio of the sound speeds, and equivalent to the reciprocal of the ratio of the indices of refraction:

$$
\frac{\sin \theta_{1}}{\sin \theta_{2}}=\frac{c_{1}}{c_{2}}=\frac{n_{2}}{n_{1}}
$$

Raytracing stems from rearranging the terms of the Law of Refraction and iterating for as many distinct layers as are appropriate. Given an initial launch angle, $\theta_{1},{ }^{15}$

$$
\frac{\cos \theta_{1}}{c_{1}}=\frac{\cos \theta_{2}}{c_{2}}=\frac{\cos \theta_{z}}{c_{z}}
$$

\footnotetext{
${ }^{15}$ In ocean acoustics, we use the ratio of cosines, with the reference angle to the vertical
} 
Any individual ray "traces" through the medium, bending as it maintains the ratio between the cosine of the angle and the sound speed in that layer. We can extend this for an infinite amount of rays coming from a point source ensonifying the water column. While a simple explanation for sound propagation, when implemented properly, it is a powerful tool for a variety of ocean environments. A robust computational solution for this implements nonlinear differential equations, which are well explained in the Computational Ocean Acoustics textbook. $^{16}$

This thesis primarily uses ray tracing methods for the forward problem because of the environmental dynamics and computational constraints posed by field operations. In particular, ray tracing is a preferred computational method because of its: ${ }^{17}$

1. relatively efficient computational time, to run onboard a vehicle

2. intuitive handling of travel time, to undergird range estimation

3. sensitivity to minute environmental changes, to improve range estimation accuracy

4. parametric nature, to solve for a subset of launch angles or for the arrival times of a high-resolution sub-grid

5. easy integration for the Doppler effect, to account for vehicle movement

6. applicability for higher frequency sources which we use for ICEX20

\subsection{Post-PRocessing ACOUSTIC POSITIONING}

One natural application of ray tracing is range estimation, a the necessary input for creating an underwater equivalent to GNSS. This is the opposite problem that Colladon and Strum

\footnotetext{
${ }^{16}$ Jensen et al., Computational Ocean Acoustics.
}

${ }^{17}$ Ibid. 
(1826) solved for when measuring the speed of sound underwater. The literature on navigation and localization, especially from the ocean acoustics community, is loose with regard to post-processing versus real-time capabilities and challenges. Accordingly, I separate the literature between post-processing and in situ results. Generally speaking, the acoustic community only considers a depth-dependent SSP in post-processing via labor intensive acoustic modeling and/or bespoke algorithms. The practice for in situ processing, or field deployments, simplifies the sound speed to a deterministic calculation, with a known sound speed and standard deviation, similar to GNSS. The work in this thesis, to my knowledge, is the first to autonomously leverage an acoustic model for range estimation in real time.

\subsubsection{UNKNOWN SOURCES}

Some implementations of localization focus on unknown sources, like biophony or geophony.

Famously, the Sound Surveillance System (SOSUS) listening stations tracked a 43 day record of an individual blue whale as it took a $3200 \mathrm{~km}$ tour in the northern Atlantic. The whale vocalized at a rate of one "comma" every 74 seconds for most of those 43 days, which built rapport with Lt. George Gagnon, who nicknamed it "Ol’ Blue”. SOSUS also monitored earthquakes, where an underwater hydrophone can detect at least 10 times more earthquakes than a comparability situated land-based seismometer. ${ }^{18}$

Tiemann et al. analyzed humpback whale songs on six widely spaced receivers near Hawaii, and used a ray acoustic propagation model to account for the waveguide, multipath, and bathymetric effects to estimate travel time from hypothesized whale positions. The whale calls were localized using a time-difference of arrival between receiver pairs. ${ }^{19}$ This method was also replicated to identify where fish inhabit, a fundamentally important topic in ecology and man-

\footnotetext{
${ }^{18}$ Nishimura, Monitoring whales and earthquakes by using SOSUS..

${ }^{19}$ Tiemann et al., "Localization of marine mammals near Hawaii using an acoustic propagation model".
} 
agement, which can prioritize acoustically sensitive times and/or areas. A particular study found the uncertainty to be less than 2 meters for boatwhistles from toadfish in Eel Pond, Woods Hole. ${ }^{20}$

An example from the Beaufort Sea used ray tracing to identify discrete ice cracking events. ${ }^{21}$ The main angle of incidence was found by beamforming on a vertical line array; rays were traced out with a narrow set of launch angles with a depth dependent sound speed. These rays interact with the surface at distances that line up with satellite data showing an ice pressure ridge.

It should also be noted that range estimation is possible via normal modes, with modal back propagation, but this method becomes inapplicable for higher frequencies for which rays are much better suited. ${ }^{22}$ There are also "unknown" anthropogenic sources of opportunity, like ships, where applications of tomography jointly estimate the sound speed and the positions of the sources. $^{23}$

\subsubsection{KNOWN SOURCES}

Many localization applications focus on known sources, such as vehicles, buoys, beacons, etc., over short and long ranges. This overview of known sources predominantly focuses on floats and gliders, as these tend to be low power consumption and generate ripe datasets for postprocessing localization. AUVs, on the other hand, will be discussed in greater detail in section 2.4 .

\footnotetext{
${ }^{20}$ Putland et al., "Localizing individual soniferous fish using passive acoustic monitoring".

${ }^{21}$ Chen et al., "Temporal and spatial characteristics of the Beaufort Sea ambient noise environment".

${ }^{22}$ Newhall et al., "Long distance passive localization of vocalizing sei whales using an acoustic normal mode approach".

${ }^{23}$ Cornuelle et al., "Ocean acoustic tomography from ships"; Gaillard, "Ocean acoustic tomography with moving sources or receivers"; Verlinden et al., "Passive acoustic tracking using a library of nearby sources of opportunity".
} 
In 1970, Rossby and Webb deployed two Swallow floats in the SOFAR channel. ${ }^{24}$ Signals from these floats were received by SOSUS hydrophones and recorded continuously, archiving the time of arrival. The first float had a distance of $846.5 \mathrm{~km}$ according to the LORAN A fix $^{25}$ and a distance of $843.4 \mathrm{~km}$ according to the acoustic travel time assuming a mean sound velocity of $1492 \mathrm{~m} / \mathrm{s}$ in the SOFAR channel. Similarly, the acoustically determined position for the second float was $5 \mathrm{~km}$ off the LORAN A fix. Interestingly, the authors consider this a large error (it's less than one percent) and attribute it to LORAN A, not the acoustic measurement or sound speed variability.

Eventually, equipping each float with an acoustic source was time- and cost-prohibitive. The SOFAR floats were reversed in design such that the float had the hydrophone instead; this version was playfully named the RAFOS floats. ${ }^{26}$ The RAFOS floats held a CMOS microprocessor for acoustic signal detection and storage. The RAFOS sources, at $260 \mathrm{~Hz}$, have been useful to track floats in open water, but the float is unable to get a GPS fix when there is sea ice present. A study tracking 22 floats under the ice in the Weddell Gyre near Antarctica showed that they were unable to surface for 8 months. Position uncertainty grew to $116 \pm 148 \mathrm{~km}$ in the lateral directions. ${ }^{27}$

In 2006, Duda et al. proposed a RAFOS-2 float tracking and thermometry system. ${ }^{28}$ They propose a $50 \mathrm{~Hz}$ bandwidth system from 100 to $200 \mathrm{~Hz}$ which would double the useful range, quadruple the coverage area of each source in a navigation net, but also constrain the float position with a precision of $50 \mathrm{~m}$ or better. Thus this network of sources could transmit signals to many floats, and would be capable of computing meaningful 3-dimensional volumetric estimates of heat content and mapping ocean eddies.

\footnotetext{
${ }^{24}$ Rossby et al., "Observing abyssal motions by tracking Swallow floats in the SOFAR channel".

${ }^{25}$ LORAN A is a hyperbolic radio navigation system

${ }^{26}$ Rossby et al., "The RAFOS System".

${ }^{27}$ Chamberlain et al., "Observing the Ice-Covered Weddell Gyre With Profiling Floats".

${ }^{28}$ Duda et al., "Evaluation of a Long-Range Joint Acoustic Navigation / Thermometry System".
} 
An experiment in the Phillipine Sea featured six moored acoustic sources arranged as a pentagon (one in the middle), creating a rich dataset for long-range positioning algorithms. The sources were roughly $400 \mathrm{~km}$ to a side and collected data from 2010 to 2011 . There are a few notable localization papers that came out of this dataset, which are reviewed here.

Four autonomous underwater gliders (AUGs) with acoustic modems were deployed from November 2010 to April 2011 within the Phillipine Sea tomographic array. ${ }^{29}$ The AUGs recorded acoustic transmission from the moored sources up to $700 \mathrm{~km}$ away; these signals, when recorded from five or more sources, were used to estimate the position of a glider when it was underwater. While underway, the gliders self-positioned using a kinematic model and intermittent GPS fixes. AUGs move much slower than AUVs throughout the water column; these averaged underwater missions of 6.4 hours over $3.6 \mathrm{~km}$, reaching depths of $1000 \mathrm{~m}$. The measured acoustic arrival peaks were unambiguously associated with predicted ray arrivals. The least squares solution for glider position estimated from acoustically derived ranges from the five sources differed from the kinematic model by 914 m, with an estimated uncertainty of 106 $\mathrm{m}$. The authors attribute roughly $70 \mathrm{~ms}(105 \mathrm{~m})$ of error due to sound speed variability in the region.

A follow up paper, that took into account three different flight models of glider subsurface motion, found similar localization discrepancies from 600-900 m between those updated kinematic models and the acoustic derived position, using events that had at least three moored source contacts. ${ }^{30}$ The estimates of post-processing error were constrained to $105 \mathrm{~m}$; further refining of data errors reduced this to $85 \mathrm{~m}$; and counterintuitively, neglecting doppler reduced this to $78 \mathrm{~m}$. A key drawback of their approach is assuming a single temporally and

\footnotetext{
${ }^{29}$ Van Uffelen et al., "Estimating uncertainty in subsurface glider position using transmissions from fixed acoustic tomography sources".

${ }^{30}$ Van Uffelen et al., "Localization and Subsurface Position Error Estimation of Gliders Using Broadband Acoustic Signals at Long Range".
} 
spatially averaged SSP for all acoustic events from November 2010 to March 2011 (ostensibly, the glider itself could have collected more reasonable sound speed data).

Another direction for range estimation in the Phillipine Sea experiment directly includes an ocean state estimate. In a model-driven method to estimate the range between a single, fixed transmitter at $998 \mathrm{~m}$ in depth and a receiver $510 \mathrm{~km}$ away and anywhere from 210-5388 m in depth, Wu et al. compared observed acoustic records with synthetic acoustic records computed through HYCOM, the Hybrid Coordinate Ocean Model. They analyze more than 8900 transmissions over 3 days to characterize the statistical variation of range errors, which unfortunately makes this difficult to compare to the aforementioned AUG studies over months. The best performing method rejects segments of the synthetic and observed records later than some critical time threshold, and then cross correlated the two to estimate range. While potentially applicable for a variety of oceanic conditions, this is reliant on model realism, and places an undue burden on computational prowess with large model products, which would not be operational in the field.

Lastly, from the Phillipine Sea dataset, an impressive algorithm presented a deep ocean, long range navigation with a "cold start" capability, that does not need any prior knowledge of the track, position, or sound speed information. ${ }^{31}$ This algorithm provides geolocation underwater without having to surface for a GPS fix, based on sources that were $129-450 \mathrm{~km}$ away from the hydrophone receivers. The receivers themselves were in a tidal watch circle up to $600 \mathrm{~m}$ in diameter. The travel time resolution afforded by the broadband source signals allows the travel time to be measured by last path/mode energy detected, which is not always the largest arrival. This latest travel time was used to isolate a single group speed with a corresponding ray. This algorithm provided position error of $85 \mathrm{~m}$ with a standard deviation of 32 $\mathrm{m}$ with respect to the ground truth GPS data on the hydrophones. The differences in group

\footnotetext{
${ }^{31}$ Mikhalevsky et al., "Deep ocean long range underwater navigation".
} 
speed between the data and from the ground truth range remain to be resolved for improving navigation performance. Implementing this algorithm in real-time remains to be seen as well.

\subsection{IN SITU ACOUSTIC POSITIONING AND NAVIGATION}

For both known and unknown sources, the range estimation or localization problem is typically done in post-processing, after all assets have been recovered, and potentially in congruence with high-resolution modeling or other data products. Underwater navigation necessitates a range estimate in real-time to drive trilateration and error tracking; because of the computational constraint of real-time calculations, that range estimate is generally oversimplified to a deterministic calculation. This deterministic calculation is valid for short ranges, at which many AUVs operate on. But it quickly breaks down for long ranges (as explored in the above section) or complex propagation environments (like the Beaufort Lens). Accurate navigation is an essential task for path planning, geo-referencing scientific observations, or co-ordination between multiple vehicle.

The traditional underwater positioning solution has an AUV stall at the surface to receive a periodic position fix from a GPS receiver. Once a vehicle descends below the water, they may rely on any combination of dead reckoning, hydrodynamic models, inertial navigation systems, doppler velocity logs, and local acoustic navigation networks. ${ }^{32}$ Positioning in the vertical direction is outsourced to a pressure sensor, which reduces the navigation problem to horizontal axes. Most autonomous vehicle deployments follow pre-determined waypoints that make an easy geometrical pattern, like a line, lawnmower, or racetrack pattern.

\footnotetext{
${ }^{32}$ Paull et al., "AUV Navigation and Localization".
} 


\subsubsection{BASELINE SYSTEMS}

Classical acoustic baseline systems use multiple distributed transponders in fixed positions to listen to vehicle requests for range. When they send a message back, the AUV can self-localize via two-way travel time ranging and trilateration. Ranging is done deterministically and is critical to the navigation; many real-time applications also include a Kalman filter to mitigate error driven by outliers. ${ }^{33}$

A long baseline (LBL) positioning system is composed of acoustic beacons, often with known positions on the seafloor. Sometimes the beacons are on the surface and utilize GPS, as is the case in this thesis. These beacons are spaced out on the order of hundreds of meters to several kilometers, and the range measurements from them have accuracies on the order of meters. The LBL system is the most similar to GNSS in terms of infrastructure.

Short baseline (SBL) systems operate as smaller LBLs, where the reference beacon is affixed to a surface vessel or small platform. Ultra-short baseline (USBL) and inverted ultra-short baseline (iUSBL) systems are even smaller, using a single transponder and an array to leverage arrival angle to triangulate position instead of time-of-flight for trilateration.

The key limitations of conventional baseline systems is the time and expense in setting up a network of beacons, and their reliance on two way travel time, which necessitates an active acoustic system on the AUV. The time and expense in setting up a network of beacons, however, is trivial compared to launching satellites for GNSS.

\footnotetext{
${ }^{33}$ Paull et al., "AUV Navigation and Localization".
} 


\subsubsection{Chip scale atomic clocks}

The technology for chip scale atompic clocks (CSACs) was initially developed for GNSS. ${ }^{34}$ The ranging accuracy in any localization effort is extremely dependent on clock accuracy, precision, and synchronization.

The CSAC is extremely useful for underwater applications; they have low power consumption (on the order of $200 \mathrm{~mW}$ ), small physical footprint (around $35 \mathrm{~g}$ and $16 \mathrm{cubic} \mathrm{cm}$ ), excellent accuracy (on the order of $10^{-10}$ seconds), and minimal drift (less than $100 \mu$ s/day). ${ }^{35}$ Thus, accurate timing is a significant capability enhancer for accurate underwater navigation. Most acoustic navigation systems rely on two way travel time ranging, with the communication cycle consisting of a vehicle-initiated pulse followed by a response from all transponders.

The chip scale atomic clock enables one way travel time (OWTT) ranging by synchronizing all clocks across assets and embedding transmit time into the message packet. The result is a direct one way travel time measurement for ranging applications. This synchronization is vital for downstream applications of sensing spatially and temporally oceanographic phenomena, particularly from multiple AUVs, as well as future development where low cost AUVs would not need an active transponder. Thus the CSAC provides scalability for multiple vehicles at increased complexity in hardware design and cost.

\subsubsection{WHOI MICRO-MODEM}

The WHOI micro-modem is a compact, low power acoustic transceiver for both acoustic communication and navigation. ${ }^{36}$ It supports the use of both broadband and narrowband transponders for LBL navigation systems. The real-time, embedded operating system is cru-

\footnotetext{
${ }^{34}$ Gardner et al., "A second look at Chip Scale Atomic Clocks for long term precision timing"; Rypkema, "Underwater \& Out of Sight: Towards Ubiquity in Underwater Robotics".

${ }^{35}$ Rypkema, "Underwater \& Out of Sight: Towards Ubiquity in Underwater Robotics".

${ }^{36}$ Singh et al., "Underwater Acoustic Navigation with the WHOI Micro-Modem".
} 
cial to OWTT ranging; the analog input signal is sampled with a 12-bit analog-to-digital converter. The passband data are demodulated and sent to a matched-field detector; a noise threshold rejects impulse noise while a detection threshold detects the peak from the matched filter output. The detector has a resolution of 125 microseconds and can be configured for a wide variety of signals to support several applications, including LBL navigation, synchronous ranging with different types of AUVs, and whale tracking and localization.

\subsubsection{REAL TIME LOCALIZATION}

The technological advancements provided by CSACs and the WHOI micro-modems have facilitated real time localization for underwater vehicles. This overview will focus on OWTT LBL techniques, as that is most similar to the acoustic part of the navigation solution for ICEX20, in loose chronological order.

Given the necessity of setting up and geolocating a network of beacons, seminal work in the early 2000s sought to improve the usability via simultaenous localization and mapping (SLAM) of the vehicle trajectory and transponder location ${ }^{37}$ or by exploring a moving LBL paradigm with transponders on surface vehicles. ${ }^{38}$

Future efforts improved the usability of LBL by introducing ${ }^{39}$ and improving ${ }^{40}$ upon singlebeacon OWTT, where OWTT range measurements accrue over time and are fused with dead reckoning to bound positional error growth.

Other work focused on making LBL navigation low cost by incorporating off the shelf sensors. The approach for one vehicle was shown as strong evidence for scalability for multiple. ${ }^{41}$

\footnotetext{
${ }^{37}$ Newman et al., "Pure range-only sub-sea SLAM".

${ }^{38}$ Vaganay et al., "Experimental validation of the moving long base-line navigation concept".

${ }^{39}$ Eustice et al., "Recent Advances in Synchronous-Clock One-Way-Travel-Time Acoustic Navigation".

${ }^{40}$ Webster et al., "Advances in single-beacon one-way-travel-time acoustic navigation for underwater vehicles", "Preliminary deep water results in single-beacon one-way-travel-time acoustic navigation for underwater vehicles".

${ }^{41}$ Melo et al., "Towards LBL positioning systems for multiple vehicles".
} 
One effort used an extended Kalman filter to coupled a consumer grade MEMS inertial measurement unit (IMU) with a vehicle dynamic model to achieve an average position error on the order of tens of meters compared to a distance traveled of 2 meters. ${ }^{42}$ A follow up work introduced a particle filter with similar accuracy in field results in Monterey Bay, California. ${ }^{43}$ Both efforts use a nominal sound speed value.

\subsubsection{UnderWater NaVigation IN THE ARCTIC}

Literature and historical reviews of underwater navigation approaches in under-ice conditions have been written by Norgren et al., ${ }^{44}$ McFarlane et al. ${ }^{45}$ and Barker et al. ${ }^{46}$ All highlight the emerging technology learning curve for vehicle operations in the Arctic, the hostile conditions brought on by permanent moving ice cover, and the need for both absolute and relative navigation with little tolerance for error. In this section I will discuss a few notable efforts in greater detail.

One of the first AUV operations in the Arctic tested launch and recovery from an ice hole. ${ }^{47}$ This effort cites "ample precedent for employing AUVs for Arctic operations" and details a specific mission chronology and vehicle design. A USBL system was used for vehicle homing, where the vehicle, equipped with barbs, entangled itself on a net suspended under the ice. This was in anticipation of an experiment a year later, in the spring of 1994, where a multipath utilization scheme was used to mitigate range inaccuracies in an LBL system. ${ }^{48}$

\footnotetext{
${ }^{42}$ Kepper et al., "MEMS IMU and One-Way-Travel-Time Navigation for Autonomous Underwater Vehicles".

${ }^{43}$ Claus et al., "Closed-loop one-way-travel-time navigation using low-grade odometry for autonomous underwater vehicles".

${ }^{44}$ Norgren et al., "Unmanned underwater vehicles in Arctic operations".

${ }^{45}$ McFarlane et al., "Autonomous Underwater Vehicle operations in the Arctic".

${ }^{46}$ Norgren et al., "Unmanned underwater vehicles in Arctic operations".

${ }^{47}$ Bellingham et al., "AUV Operations in the Arctic".

${ }^{48}$ Deffenbaugh et al., "Acoustic navigation for Arctic under-ice AUV missions".
} 
More recently, in July/August 2007, two AUVs were deployed in the Arctic Ocean's Gakkel Ridge for water-column and ocean bottom surveys. ${ }^{49}$ These surveys were the first among AUV operations to date in requiring georeferenced navigation in proximity to the seafloor beneath moving ice cover. LBL beacons were situated $150 \mathrm{~m}$ above the seafloor from a free-fall deployment from the ship where the majority of the vehicle mission was below $2000 \mathrm{~m}$, to map the seafloor. Slant ranges from the vehicle were accurately calculated using a depth-averaged sound speed.

AUVs are prime for under-ice surveys in the Arctic. A 12 day under-ice survey over 1000 $\mathrm{km}$ was accomplished in the Sever Spur. ${ }^{50}$ The operational effort consisted of both a short range positioning system, based on several modems and multilateration, and a long-range homing system, based on bearing. The navigation performance during the vehicle deployment could not be determined as their campsite was on a moving ice floe and the AUV could not be tracked when it was at the seabed. When the AUV surfaced from its $2000 \mathrm{~m}$ depth mission, the navigation drift error was considerable because there was no more DVL bottom lock during the vehicle's ascent.

Similarly, AUVs are adept at hydrographic surveys. Over eight days, a REMUS-100 AUV performed 14 kilometers of track lines for hydrographic surveys beneath a coastal ice floe offshore from Barrow, Alaska. ${ }^{51}$ The AUV navigated via LBL, and performed homing and docking behaviors via separate USBL. The acoustic navigation method here was not challenged as the local acoustic environment was approximately isovelocity and the missions were completed within a radius of $60 \mathrm{~m}$. This AUV, developed at WHOI, has been proposed for long

\footnotetext{
${ }^{49}$ Jakuba et al., "Long-baseline acoustic navigation for under-ice autonomous underwater vehicle operations"; Kunz et al., "Deep sea underwater robotic exploration in the ice-covered Arctic ocean with AUVs".

${ }^{50}$ Kaminski et al., "12 days under ice - an historic AUV deployment in the Canadian High Arctic".

${ }^{51}$ Kukulya et al., "Under-ice operations with a REMUS-100 AUV in the Arctic".
} 
range under-ice mapping of oil spills. ${ }^{52}$ Deployments in 2012 demonstrated niche observational capabilities for sensing at the margin of calving glaciers, an otherwise dangerous and undersampled environment. ${ }^{53}$ The vehicle navigated using GPS fixes at the surface and a combination of acoustic ranging from LBL transponders and dead reckoning algorithms assimilating compass data, propeller turns, water velocity and bottom track data. ${ }^{54}$

Of particular interest are AUG deployments in the Marginal Ice Zone (MIZ) in the Beaufort and Chukchi seas from July through September of 2014. A long-range acoustic navigation system with built-in acoustic communications capability was deployed for two underwater gliders. ${ }^{55}$ The transponders were suspended $100 \mathrm{~m}$ below the ice, in the Beaufort Lens lower duct, and transmitted at $900 \mathrm{~Hz}$ with an adjustable bandwidth of 25 to $100 \mathrm{~Hz}$. The timeof-flight measurements from these transponders was used to simultaneously estimate vehicle position and depth-averaged local currents. Ranges of greater than $400 \mathrm{~km}$ were achieved with range accuracy of 40 m RMS; the range error was remarkably similar between 100 and $400 \mathrm{~km}$. The real-time localization used a local measured sound speed value at the time of reception without regard to any specific rays or modes.

A later study of AUG localization in the Canada Basin, for long ranges, provides a much better comparison between real-time and post-processing localization methods. ${ }^{56}$ Broadband acoustic sources were deployed in the MIZ such that glider could still surface for a GPS fix. The two gliders were deployed in August/September of 2017, where one completed 416 dives down to $480 \mathrm{~m}$ and the other completed 296 dives to $750 \mathrm{~m}$. At the time of deployment, there

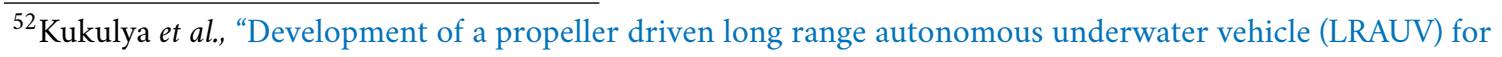
under-ice mapping of oil spills and environmental hazards".

${ }^{53}$ Stevens et al., "Linking glacially modified waters to catchment-scale subglacial discharge using autonomous underwater vehicle observations".

${ }^{54}$ Plueddemann et al., "Autonomous Underwater Vehicle Operations Beneath Coastal Sea Ice".

${ }^{55}$ Freitag et al., "Long range acoustic communications and navigation in the Arctic"; Webster et al., "Towards real-time under-ice acoustic navigation at mesoscale ranges".

${ }^{56}$ Graupe et al., "Preliminary results for glider localization in the Beaufort Duct using broadband acoustic sources at long range".
} 
was no ice coverage. Real-time ranges were estimated from measured acoustic travel times and sound speed from temperature and salinity measurements at the time of acoustic reception. The mean value for sound speed used to estimate ranges was $1450 \mathrm{~m} / \mathrm{s}$ with a standard deviation of $6.5 \mathrm{~m} / \mathrm{s}$. In post-processing, when acoustic arrival matching localization was performed, taking advantage of the position estimate provided by the kinematic model as a first order estimate for position. The error in the first method is $550 \mathrm{~m}$; the second reduces this by a factor of 4-5 depending on the dive. The variance in the factor reduction is because the acoustic arrival structure is highly dependent on the Beaufort Duct. This study notably points out that future work will incorporate acoustic arrival matching on board in the hope that post-processing results can improve realtime underwater vehicle positioning; this capability is introduced in this thesis.

\subsection{UNDERWATER VEHICLES AND THEIR AUTONOMY}

So far, the discussion of AUV capabilities, namely communication and navigation, has largely treated them like a phone relative to GPS-a computer with the ability to communicate and self-locate. But this simplification is not overly misleading; though autonomous, missions are largely pre-programmed surveys with constrained behavioral or status updates. Once a vehicle is recovered, it is reprogrammed and deployed again. Because much effort has gone into the design (including sensor payloads), control, and operations of underwater robotics, the autonomy side is a nascent yet important field.

It is important to note that the technological advancements that enabled AUVs by the early 1990s initially spurred the growth of ROVs in the 1960s-80s. ROVs revolutionized underwater exploration as they were more powerful, safer to use, and could go deeper than divers. The added infrastructure of a tether and support ship make their deployment complex, expen- 
sive, and risk-averse. A discussion on tethered remotely operated vehicles (ROVs) is outside the scope of this thesis, as they do not suffer the same navigation uncertainty nor communication limitations; more information can be found in related work ${ }^{57}$ and review papers. ${ }^{58}$ Autonomous vehicles, a natural evolution of ROVs, can re-invigorate underwater exploration by being even safer, enabling repeated access to remote places with higher-resolution data sampling, and lowering operational costs.

\subsubsection{DeSIGN}

AUVs provide spatial coverage unattainable by fixed sensors and payload flexibility at favorable costs compared to manned systems. Like many technologies in this thesis (and ocean acoustics in general), we can trace the AUV back to military and defense interests, where AUVs were birthed as UUVs-unmanned underwater vehicles. Some of the first applications of UUVs were to examine submarine wakes ${ }^{59}$ and locate lost submarines. ${ }^{60}$

Since the turn of the millennium, advancements in sensor miniaturization, lithium ion batteries, and processing power have facilitated the commercialization of AUVs, with some being specifically marketed as "low-cost". AUVs are most commonly designed like torpedoes to reduce drag, optimize power use, simplify hydrodynamic models, and improve vehicle run-time. Generally speaking, they are ballasted for slight positive buoyancy such that they rise to the surface in the case of a mission abort or system failure; some AUVs include a drop weight, connected to a burn wire, to force positive buoyancy in emergency scenarios. Given the positive buoyancy, AUVs must propel themselves forward to remain submerged.

\footnotetext{
${ }^{57}$ Rypkema, "Underwater \& Out of Sight: Towards Ubiquity in Underwater Robotics".

${ }^{58}$ Bogue, "Underwater robots"; Petillot et al., "Underwater Robots".

${ }^{59}$ Widditsch, SPURV - The First Decade.

${ }^{60}$ Walton, Evolution of a Search System.
} 


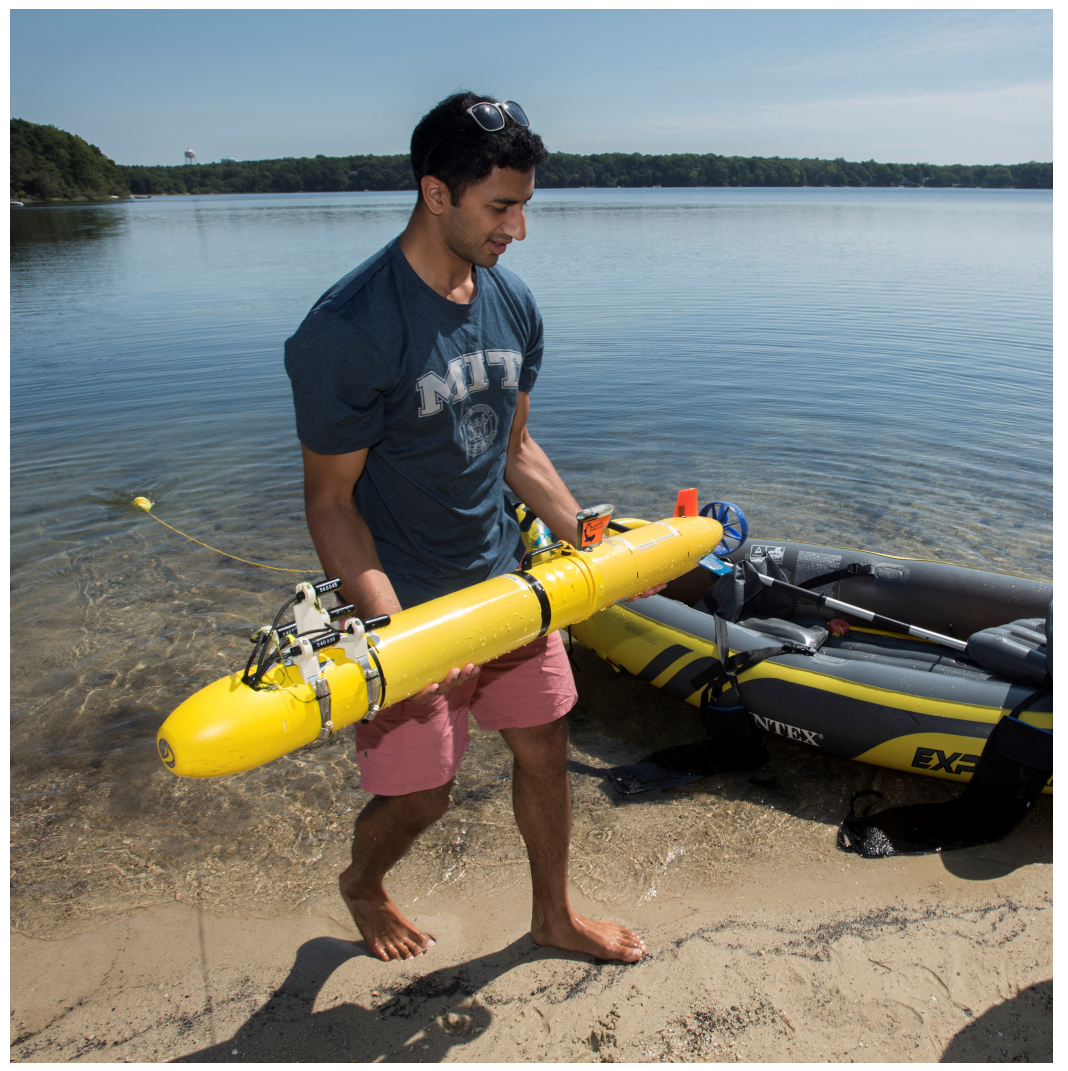

Figure 2.1: The author holding a prototype of the Bluefin Sandshark AUV with a custom hydrophone array attached, in support of fieldwork at Ashumet Pond, MA, with Nick Rypkema and Erin Fischell. Image copyrighted to Woods Hole Oceanographic Institution.

The torpedo-based AUVs come in all sizes and weights, from five to hundreds of kilograms, and require different infrastructure for field operations. Two of the most successful AUV platforms are Bluefin ${ }^{61}$ (owned by General Dynamics) and the REMUS ${ }^{62}$ (owned by WHOI and a spinoff company, Hydroid Inc.). The products in their respective series are marked by a number; the former platform indicates diameter (in inches) whereas the latter indicates maximum operating depth.

One of the smallest, but no longer available, is the low-cost Bluefin Sandshark (as shown in figure 2.1) with a $12.4 \mathrm{~cm}$ diameter and $51 \mathrm{~cm}$ length; it is easily operated by one person,

\footnotetext{
${ }^{61}$ https://gdmissionsystems.com/underwater-vehicles/bluefin-robotics

${ }^{62}$ https://www.whoi.edu/what-we-do/explore/underwater-vehicles/auvs/remus/
} 
and can swim at 2-4 knots and dive down to $200 \mathrm{~m}$. Currently available small AUVs, which necessitate at least two people to operate, are the Bluefin-9, REMUS 100, and IVER3 from L3Harris $^{63}$; these operate down to 100 to $200 \mathrm{~m}$ for 8 to 14 hour missions.

The Bluefin-12 is a smaller mid-sized AUV, with a $200 \mathrm{~m}$ depth rating but with 24 to 36 hour endurance. The REMUS 600, a larger mid-sized AUV, has similar endurance with the option for a $1500 \mathrm{~m}$ depth rating as well. These have lengths from 3 and $5 \mathrm{~m}$, and weight 220 and $385 \mathrm{~kg}$, respectively.

Large AUVs, such as the Bluefin-21 and REMUS 6000, have deep water ratings with twice the weight and similar endurance as the mid-sized AUVs. Overall, most commercial AUVs by default integrate a GPS antenna (along with Iridium or a strobe), inertial navigation system, CTD (or CT) sensors, and doppler velocity loggers; all use a propeller for propulsion and boast a modular design for any other payload packages. The dive limit and endurance, in particular, place physical constraints on mission scope or autonomous behavior.

While work in this thesis does not use (AUGs), their applications are referenced often enough to warrant discussion around their design. In a now famous and visionary 1989 article, Henry Stommel introduced the system of a fleet of gliders-he calls them autonomous profiling floats, driven by temperature differences-that report back to Mission Control via satellite six times a day, use no fuel, and collect unprecedented CTD data throughout the water column. ${ }^{64}$ At the time, AUGs presented a creative solution for where ROVs and AUVs could not go, both in depth and length of mission. AUGs are low powered, long duration, and relatively inert. ${ }^{65}$ Their missions tend to be on the order of months to years, and hundreds to thousands of kilometers. They provide an extensive look at the mid-water column, but their lower power consumption limits the types of sensors and sampling frequency. Overall, the relative

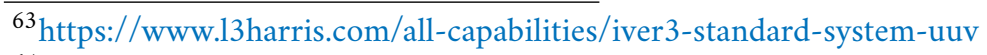

${ }^{64}$ Stommel, "The Slocum Mission".

${ }^{65}$ Schofield et al., "Slocum Gliders"; Sherman et al., "The autonomous underwater glider "Spray"”.
} 
simplicity of the platform is its brilliance, but also limits self-navigation, communication, and adaptive sensing that AUVs provide.

\subsubsection{Autonomy}

The trend in marine robotics, particularly for applications in remote and/or dangerous conditions, is to transition from individual robots operated by teams at large expense to teams of cheaper robots collaborating under minimal human supervision. ${ }^{66}$ The catalyst for this transition is not in hardware but in software-autonomy is a nascent field aided by advances in data storage, onboard computing power, and artificial intelligence. Architectures suitable for realtime applications are the Mission Oriented Operating Suite (MOOS), ${ }^{67}$ the Robot Operating System (ROS), ${ }^{68}$ and the Lightweight Communications and Marshalling library (LCM). ${ }^{69}$

In this transition, we first consider what it might mean for an individual robot to have autonomy. Shifting some decision-making power to the robot is a natural solution to the acoustic communication bandlimited environment-there is less information to send if the vehicle needs less supervision. In particular, MOOS inculcates vehicle intelligence through Interval Programming (IvP), such that a multi-objective optimization engine is used to produce a compromise between many (and potentially competing) autonomous behaviors. Coupled with a publish-and-subscribe architecture, information is shared across any behavior that requires it. This design allows for a fairly plug-and-play approach for easy interoperability and scalability.

In contrast to single AUV deployments, which already provide better spatial coverage and flexibility than fixed sensors, multiple AUV deployments reduce spatial and temporal aliasing for oceanographic sampling and can also be more efficient for time-bounded missions such as

\footnotetext{
${ }^{66}$ Bellingham et al., "Robotics in Remote and Hostile Environments".

${ }^{67}$ Benjamin et al., "Nested autonomy for unmanned marine vehicles with MOOS-IvP".

${ }^{68}$ Quigley et al., "ROS: an open-source Robot Operating System".

${ }^{69}$ Huang et al., "LCM: Lightweight Communications and Marshalling".
} 
target detection. The added complexity of multi-vehicle-potentially heterogenous-collaboration necessitates collaborative autonomy that builds on self-autonomy. Information that was previously shared between behaviors on one robot can be shared between different robots (and their behaviors), or to a topside mission command node. Yet, given the advances in data storage, memory, and computational processing on AUVs, even a small mission can collect an extraordinary amount of data via sensor sampling and logging behaviors. The sheer volume of this output far exceeds what could be telemetered via acoustic communication; collaborative autonomy begins with lightweight communication.

The challenges in navigation and collaboration reside in using the same limited-bandwidth physical medium for both processes in addition to the added complexity of field operations. Much progress in autonomy is made in small-scale efforts or high-fidelity virtual experiments.

\subsection{ENVIRONMENTALLY ADAPTIVE AUTONOMY}

One key application of autonomous behaviors includes environmental adaptation, a key underpinning for both acoustic communication (which is sensitive to environmental fluctuations) and oceanographic sampling (to accrue a useful and potentially unprecedented dataset). But given the constraints of onboard computation and data storage, satellite data or realistic ocean models are not easily integrated onboard the vehicle for real-time operations. There has been considerable work to approach and optimize the information density problem for environmentally adaptive behaviors, which will, given the spatio-temporal nature of ocean processes, be paramount to robust autonomy. The following subsections first introduce the spatial and temporal scales of various ocean features of interest and then review methodologies in the literature to inform vehicle operations. 


\subsubsection{Spatio-temporal SCALES of ocean fEatures}

Though many vehicle operations consider a static or a "snapshot" of the ocean, the spatial and temporal dynamics are worth contextualing with respect to the mission time and sampling paradigms. Oceanographic features are nominally classified on the horizontal length scale which dwarfs the vertical length scale. There are small-scale (less than $10 \mathrm{~km})$, mesoscale (10$100 \mathrm{~km}$ ), and large-scale (greater than $100 \mathrm{~km}$ ). Intuitively, greater length scales correlate with longer temporal scales, as shown in figure 2.2. ${ }^{70}$ The work in this thesis, particularly Chapter 3, uses an abundance of small-scale point sampling to draw a portrait of a mesoscale feature, the Beaufort Lens.

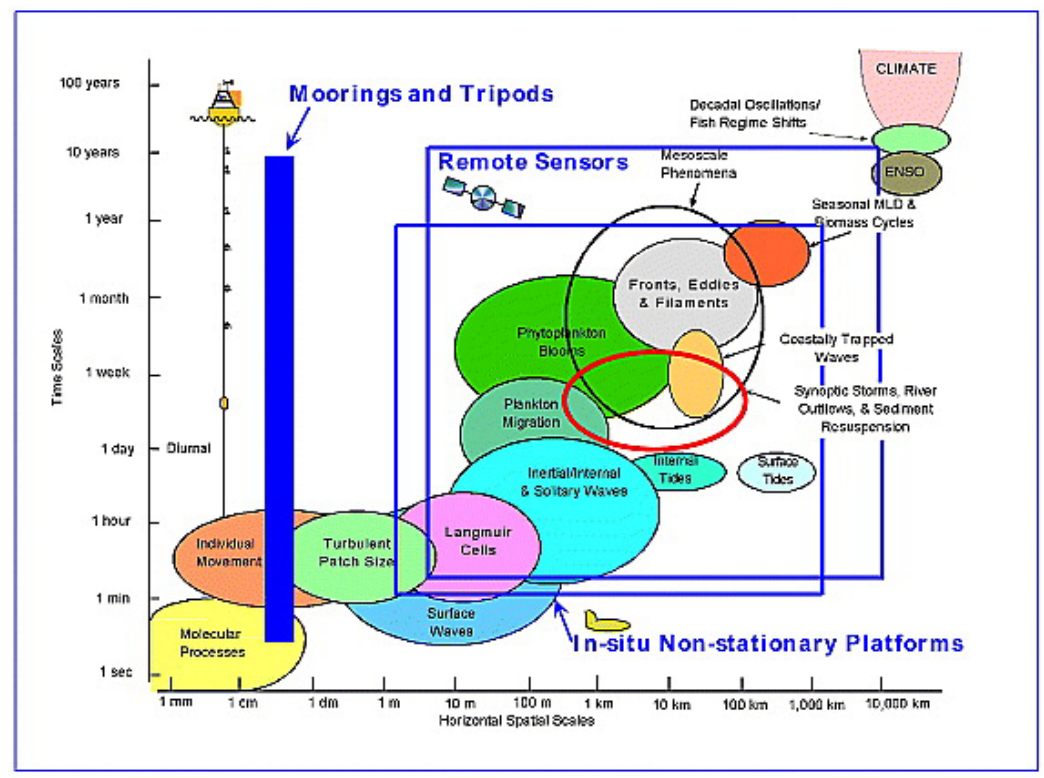

Figure 2.2: Spatial and temporal scales of ocean features with superimposed sampling domains of various platforms (moorings, satellites, AUVs, etc.) from Dickey et al. $(2001,2006)$.

Notably, ocean acoustics, even for long range applications, are much more sensitive to vertical than horizontal variability. The idea of a "range-independent" SSP assumes a dominant acoustic propagation environment for a region of interest. While the range dependence of

\footnotetext{
${ }^{70}$ Dickey et al., "Optical oceanography"; Dickey, "The Role of New Technology in Advancing Ocean Biogeo-
} chemical Research". 
any sound speed environment is a function of the relevant temporal and spatial scales, the significance of these fluctuations is an active field of interest, and predicting these fluctuations is significantly complex. Features like internal waves, tides, fronts, eddies, and filaments are most relevant for the environmental perturbations that impact acoustic propagation. Many acoustic experiments operate on moorings and thus see these features pass by with incredible temporal resolution but little spatial coherence. Non-stationary platforms like AUGs and AUVs, especially when deployed in a team, can provide much better sampling without aliasing.

\subsubsection{OPERATIONAL APPROACHES FOR ENVIRONMENTAL ADAPTION IN THE}

\section{LITERATURE}

Due to the extreme data aggregation for acoustic experiments (GBs to TBs) and the computationally intense nature of modeling acoustics, many studies examine environmental variability through post-processing data, often on much wider temporal and spatial scales. While there are many different flavors of these environmentally adaptive approaches, they loosely evolve across these ideas: data assimilation; rapid modeling; parameter estimation; and through the sensor modalities.

The basic concept of data assimilation is to combine real observations with numerical models to provide a better estimate, in this case, of physical quantities in the ocean. ${ }^{71}$ Four-dimensional data assimilation via an error subspace statistical estimation (ESSE) demonstrated how to couple physical uncertainties with acoustical ones, and then decompose the dominant acoustical error statistics. ${ }^{72}$ The method couples data, such as CTD and velocity profiles, and remote sensed sea surface height and temperature, to the physical dynamics by measurement models. While not feasible for real-time processing, the effort paints a clear picture of the abundance

\footnotetext{
${ }^{71}$ Wang et al., "Data assimilation and its applications".

${ }^{72}$ Lermusiaux et al., Four Dimensional Data Assimilation for Coupled Physical-Acoustical Fields.
} 
of data necessary to perform full field estimations. Later efforts used data assimilation for prediction systems, ${ }^{73}$ with an even more holistic end-to-end system: coupling meteorology, physical oceanography, geoacoustics, ocean acoustics, and sonar performance to estimate collective uncertainties, transfers, and their feedbacks. This approach was demonstrated with an intensive data effort in the Mid-Atlantic Bight, ultimately finding transmission loss uncertainties for a broadband sonar equation. These previous efforts set the stage for rapid data assimilation and modeling approaches to be tested in simulations.

The Adaptive Rapid Environmental Assessment (AREA) framework minimized the nonmodel-based sonar performance prediction uncertainty and improved the model-based sonar performance by rapid and adaptive in situ measurement. ${ }^{74}$ This framework was extended to adaptive path planning, where the computation of routes was optimized to characterize the environmental and acoustic ocean fields given the constraints of the observing network. ${ }^{75}$ Simulations demonstrated multi-vehicle and multi-day path planning in the Monterey Bay region $^{76}$ and in the northern Tyrrhenian Sea. ${ }^{77}$ The combined AREA-ESSE framework uses real-time oceanographic and acoustic forecasts ${ }^{78}$ as inputs to a path planning optimization scheme to seek parameter values for sampling behavior; optimal parameters were fed to the sampling vehicles as priors, with an onboard capability to re-route the path by modifying those parameters. $^{79}$

\footnotetext{
${ }_{73}^{73}$ Robinson et al., "Prediction Systems With Data Assimilation for Coupled Ocean Science and Ocean Acoustics".

${ }^{74}$ Wang, "Adaptive Rapid Environmental Assessment System Simulation Framework".

${ }^{75}$ Yilmaz et al., "Path planning methods for adaptive sampling of environmental and acoustical ocean fields".

${ }^{76}$ Wang, "Autonomous Underwater Vehicle Path Planning and Adaptive On-board Routing for Adaptive Rapid Environmental Assessment"; Yilmaz et al., "Path Planning of Autonomous Underwater Vehicles for Adaptive Sampling Using Mixed Integer Linear Programming”.

${ }^{77}$ Wang, "Autonomous Underwater Vehicle Path Planning and Adaptive On-board Routing for Adaptive Rapid Environmental Assessment".

${ }^{78} \mathrm{Lam}$ et al., "At-sea real-time coupled four-dimensional oceanographic and acoustic forecasts during Battlespace Preparation 2007".

${ }^{79}$ Wang et al., "Acoustically focused adaptive sampling and on-board routing for marine rapid environmental assessment".
} 
These path planning via data assimilation methods, also demonstrated for internal wave classification, ${ }^{80}$ path planning with a vehicle dynamical model, ${ }^{81}$ and nutrient flux, ${ }^{82}$ require intensive computational efforts or large data products, like recent satellite imagery. Recent work sought to remove the emphasis on significant prior knowledge or models of the environment, decouple the environmental and acoustic fields, but place the burden of computation and environmental assessment on an AUV. ${ }^{83}$ In the Autonomous Adaptive Environmental Assessment (AAEA), AUVs swim through a hydrographic environment and characterize it in real-time, effectively detecting features of interest. This capability is developed to track a thermocline in the Tyrrhenian Sea and in Lake Champlain, Vermont. ${ }^{84}$ Other efforts, in virtual experiments, refined the search methods for three-dimensional front tracking and hydrothermal vents. ${ }^{85}$

More recently, Fossum et al. showed an information-driven approach to robotic sampling in the coastal ocean. They rely on ocean models but use Gaussian process modeling and objective functions to facilitate sampling in regions of high scientific interest. The results from both simulation and field trials, off the coast of Norway, showed that the algorithms differentiated between alternative survey strategies and utilized correspondence between an ocean model and an AUV to determine the thermocline shift from an influx of Atlantic Water. ${ }^{86}$

But environmental autonomy is often still considered an engineering boundary to push as opposed to a standard sensing paradigm on AUVs. A recent study to catalog the heat content

\footnotetext{
${ }^{80}$ Zhang et al., "Spectral-Feature Classification of Oceanographic Processes Using an Autonomous Underwater Vehicle".

${ }^{81}$ Hover, "Path planning for data assimilation in mobile environmental monitoring systems".

${ }^{82}$ Das et al., "Simultaneous tracking and sampling of dynamic oceanographic features with autonomous underwater vehicles and lagrangian drifters".

${ }^{83}$ Petillo et al., "Autonomous Adaptive Environmental Assessment and Feature Tracking via Autonomous Underwater Vehicles".

${ }^{84} \mathrm{Ibid}$.

${ }^{85}$ Petillo, "Autonomous \& Adaptive Oceanographic Feature Tracking On Board Autonomous Underwater Vehicles".

${ }^{86}$ Fossum et al., "Information-driven robotic sampling in the coastal ocean".
} 
underneath troughs in Thwaites glacier, Antarctica, used an AUV to get an unprecedented survey of oceanographic parameters (temperature, salinity, oxygen content, and current velocity) with multi-beam data on a pre-planned track at a set altitude. ${ }^{87}$ When the vehicle performed transects between the troughs, it operated at a deeper constant depth, and observed a warmer water layer. AUVs provide an important and unique set of sensing capabilities; developing their embedded intelligence to maintain trust for an end user and produce datasets of high scientific value remains an important endeavor.

Much of real-time environmentally adaptive autonomy has focused on environmental characterization; as it has become more robust, it can fold back into other relevant domains for real-time operations. Acoustic dependent functionalities like communication and navigation, which some of the early research tackled by coupling physical and acoustic modeling, will be the next challenge for onboard data assimilation going forward. ${ }^{88}$

\subsection{Summary}

This chapter introduces key concepts across many connected fields. A quick summary of their relevance for this dissertation is distilled here:

1. Global Navigation Satellite System-GNSS is essential for terrestrial drone navigation but does not work beneath the surface of the ocean, due to electromagnetic waves being quickly absorbed. The technology and design principles around GNSS set the foundation for and enable advances in underwater acoustic navigation. This work is motivated by demonstrating GNSS-like navigation accuracy and precision for an AUV in an under-ice environment.

\footnotetext{
${ }^{87}$ Wåhlin et al., "Pathways and modification of warm water flowing beneath Thwaites Ice Shelf, West Antarctica".

${ }^{88}$ Moore et al., "Synthesis of ocean observations using data assimilation for operational, real-time and reanalysis systems: A more complete picture of the state of the ocean".
} 
2. Post-processing acoustic localization-The ocean acoustics community is broadly interested in localizing known and unknown sources in a variety of acoustic propagation environments. The key element of localization is ranging from multiple sources to trilaterate for a point of origin. These methods are predominantly in post-processing, and combine the data taken with high-resolution modeling, contemporaneous data sets, and/or computationally-intensive algorithms. Advanced acoustic localization methods consider complex features such as the spatio-temporal dynamics, surface scattering, internal waves, or hydrodynamical models to mitigate navigation error. The literature presents error as the difference between various methods, which is often posed as simple versus advanced; this error is on the order of hundreds of meters to kilometers. Because of the challenges of undersea operations, these experiments rarely provide actual ground truth via GPS for comparison. This work retools range estimation methods only used in post-processing, for real-time under-ice operations. This work also leverages an experiment design of GPS-tracked acoustic events between beacons to verify range estimation.

3. In situ localization-Localization that happens in real-time for AUV or AUG operations is navigation. Acoustic navigation is dependent on the one or two way travel time between nodes, which are converted to range estimates. Because of the data and computational constraints of real-time field operations, real-time applications have always used a deterministic sound speed value, with some being driven by local samples. This capability is often used to geo-locate physical observations, and while effective for small ranges, introduces large error for longer ranges and/or acoustically complex environments. This work demonstrates real-time navigation for an AUV in an under-ice 
environment using a re-tooling of acoustic arrival methods generally deemed too labor-intensive.

4. Underwater vehicles and their autonomy-The first AUVs had basic levels of autonomy and could execute strictly pre-planned missions. Now, more complex autonomy has introduced adaptive sampling, communication, navigation, and safety behaviors. Autonomy began with how an individual robot can balance different, and sometimes competing outcomes; acoustic communication catalyzes autonomy into collaborative, multi-vehicle behaviors that can improve spatial coverage and performance in timebounded tasks. This work builds on decades of research in the MIT LAMSS ecosystem such that the AUV Macrura, a heavily modified Bluefin-21 running MOOS-IvP, could perform environmentally adaptive navigation and communication behaviors in the New Arctic.

5. Environmentally adaptive autonomy-Originally driven by data assimilation paradigms passing uncertainties from physical models and measurements to acoustical models and measurements, advances in data storage and processing power transitioned towards real-time and at-sea applications for path planning, sonar performance prediction, and transient oceanographic feature detection. The maturation of the AUV transitioned some of that computational labor onto the AUV itself, enabling capabilities like feature characterization and tracking. But environmentally adaptive autonomy is only as good as the models and data it digests; it is only as trusted as it is transparent for a human operator. This work, given the lack of accurate modeling for the Beaufort Lens, designs and implements a data forward, human-in-the-loop Virtual Ocean decision framework to update a locally changing sound speed estimate across all submerged assets via acoustic communications. 


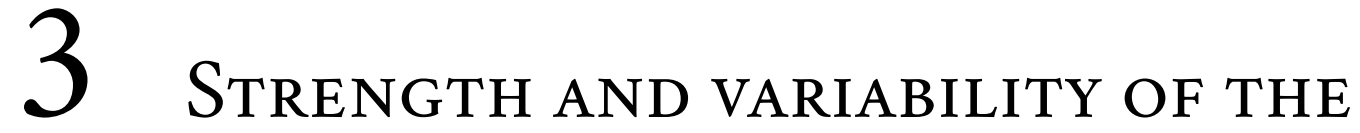

\section{BEAUFORT LENS}

We shall not cease from exploration, and the end of all our exploring will be to arrive where we started and know the place for the first time

T.S. Eliot, Little Gidding (1942)

The Beaufort Lens presents a drastically different acoustic environment than is traditionally observed in the Arctic. In the context of challenges it created during ICEX16, the work in this chapter seeks to better know the Beaufort Lens for vehicle operations in ICEX20, where acoustic communication and navigation are vital to mission success. Thus this chapter is not so much looking at the Beaufort Lens as an oceanographic feature of interest but as a numerical feature with a complicated data story. The jargon here and the success of this exploratory data analysis will be woven into Chapters 4 and 5, for its implementation in the Virtual Ocean and significance to vehicle navigation in ICEX20, respectively. But perhaps another dimension to evaluate this work is its interoperability for future vehicle deployments in the Arctic. If the data pipeline developed here simplifies the work for any other roboticists and/or oceanographers, that is its own kind of success. In the spirit of building upon previous technologies, the data-forward, statistical integration of the Beaufort Lens for vehicle operations is not possible without the WHOI Ice Tethered Profiler program. 


\subsection{WHOI Ice Tethered Profilers}

The WHOI Ice Tethered Profiler was specifically designed as an autonomous instrument for sustained observation in the Arctic Ocean, inspired by the successful Argo profiler. ${ }^{1}$

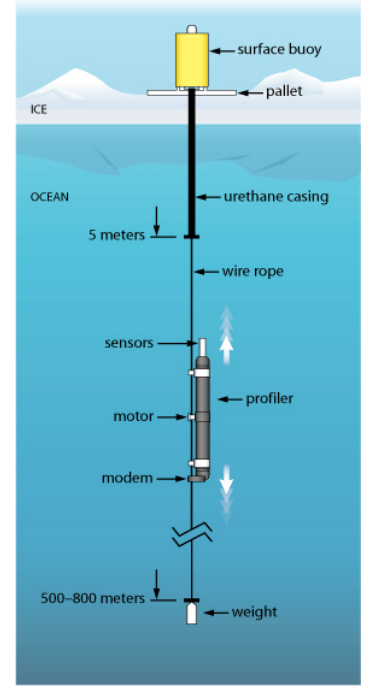

(a) Engineering schematic of the ITP profiler

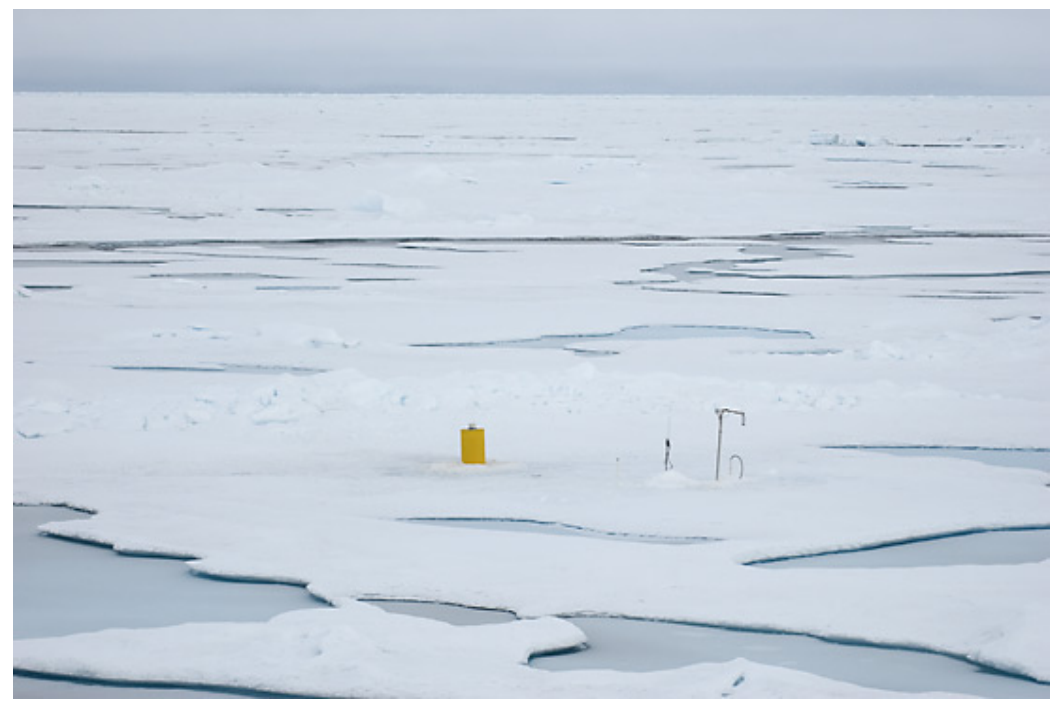

(b) An image of a single ITP buoy (in yellow) in an ice floe, courtesy of Chris Linder (WHOI)

Figure 3.1: Overview of the Woods Hole Oceanographic Institution's Ice Tethered Profiler platform. The Ice-Tethered Profiler data were collected and made available by the Ice-Tethered Profiler Program (Toole et al., 2011; Krishfield et al., 2008) based at the Woods Hole Oceanographic Institution www.whoi.edu/itp.

As shown in figure 3.1, a GPS-enabled surface buoy sits atop an ice floe, supporting a lengthy weighted wire underwater. A profiling instrument cycles vertically along this wire, generally down to $750 \mathrm{~m}$, recording conductivity and temperature (and sometimes oxygen concentration) with respect to pressure. This data is serialized, compressed, and telemetered to shore in near real-time. The ITP program creates a dataset that is spatio-temporally sparse but presents the state-of-the-art technology for surveying extreme polar environments consistently and

\footnotetext{
${ }^{1}$ Krishfield et al., "Automated Ice-Tethered Profilers for Seawater Observations under Pack Ice in All Seasons"; Toole et al., "The Ice-Tethered Profiler".
} 
robustly. At the time of writing, there have been 121 ITPs deployed, where missions last anywhere from a few months to a couple years.

\subsection{DATA PROCESSING}

Many studies using ITP data employ either a single representative observation ${ }^{2}$ or observations with respect to the profiler (e.g., ITPs 3, 14, and 15) and the path they take. ${ }^{3}$ The data assimilation framework introduced in this thesis, used to seed simulation cases in Chen et $\mathrm{al}^{4}{ }^{4}$ is agnostic to the profiler itself. It assimilates each observation to a desired depth grid, and treats each profile as an independent observation with a time, latitude, and longitude. This framing facilitates a more comprehensive look at information density throughout the gyre, first through a map of all the ITP data in the Beaufort Gyre from 2006 to 2020, shown in figure 3.2. The 74,863 profiles captured in this span form a distribution most concentrated in the middle of the gyre, with low sampling density throughout the Canada Basin. The two ice camps for ICEX16 and ICEX20 are in regions of low information density.

The real-time nature of ITP data necessitates a quality assurance scheme. Tier 1 is raw, from the sensor package itself; tier 2 is compressed, telemetered to shore in one format; tier 3 is the final data, polished and cleaned, in another format. Tier 3 data is assembled once the profiler is recovered, introducing a complicating latency factor for the duration of the profiler,

\footnotetext{
${ }^{2}$ Carper, "Low frequency active sonar performance in the Arctic Beaufort Lens"; Chen et al., "Spectral, spatial, and temporal characteristics of underwater ambient noise in the Beaufort Sea in 1994 and 2016"; Duda, "Acoustic signal and noise changes in the Beaufort Sea Pacific Water duct under anticipated future acidification of Arctic Ocean waters"; Poulsen et al., "Acoustic noise properties in the rapidly changing Arctic Ocean"; Schmidt et al., "Acoustic Communication and Navigation in the New Arctic - A Model Case for Environmental Adaptation".

${ }^{3}$ Timmermans et al., "Ice-Tethered Profiler observations of the double-diffusive staircase in the Canada Basin thermocline"; Timmermans et al., "Horizontal Density Structure and Restratification of the Arctic Ocean Surface Layer", "Warming of the interior Arctic Ocean linked to sea ice losses at the basin margins"; Toole et al., "Influences of the ocean surface mixed layer and thermohaline stratification on Arctic Sea ice in the central Canada Basin".

${ }^{4}$ Chen et al., "Temporal and spatial characteristics of the Beaufort Sea ambient noise environment".
} 


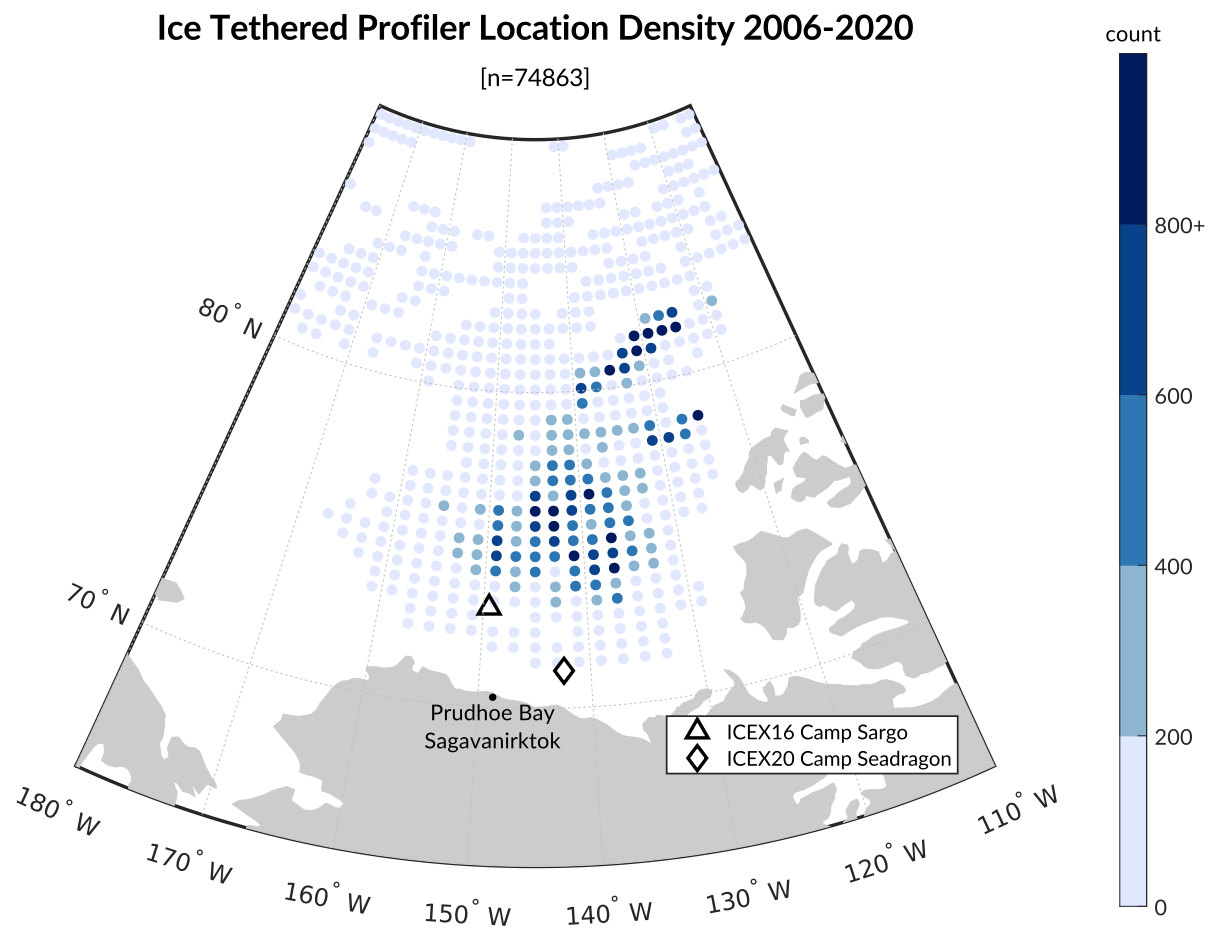

Figure 3.2: A map of ITP location density for the 74,863 profiles from 2006 to 2020 in the Beaufort Gyre (the bottom part of this map shows Northern Alaska). Each dot represents a bin of 2 degrees longitude by 0.5 degrees latitude. The darker blues show where more profilers have drifted and successfully collected data. The black triangle and diamond show the ice camp locations for ICEX16 and ICEX20, respectively.

especially for the purpose of using the near real-time data to inform field deployments. Thus, the data pipeline in this thesis performs its own quality checks for the minimum, maximum, and standard deviation of physical parameters found, and also re-assimilates any profiles with non-monotonically increasing depth data. Lastly, the framework developed can automatically update a local copy of the ITP data with any new data posted to the WHOI server. This automation simplifies the maneuverability and usefulness of the data for further analysis. ${ }^{5}$

${ }^{5}$ GitHub: https://github.com/eeshanbot/itp-articuno 


\subsection{Background on the Beaufort Sea}

The Arctic is an extremely sensitive region to anthropogenic climate change. Amidst changes in sea ice cover and warming surface temperatures, there has been a departure from the characteristic SSP in the Arctic. The classical Arctic ocean SSP is monotonically increasing, so rays refract upward and interact with the ice. The Beaufort Lens presents a double ducted propagation environment, with channels above and below the knee. The surface channel, rays experience minimal transmission loss but signal coherence degrades due to higher frequency interaction with sea ice. The lower channel traps sound above $300 \mathrm{~Hz}$ for near lossless mesoscale propagation.

There has been a relatively recent focus on acoustic studies in and of the Beaufort Lens with various aims, data sets, and techniques. While something akin to the Beaufort Lens was first observed in $1970,{ }^{6}$ its impact on acoustic propagation was negligible. ${ }^{7}$ As a note, the literature interchangeably refers to this phenomena as a tongue, a knee, and a duct. We clarify that the lens, tongue, and knee are oceanographic features describing the local sound speed maxima driven by warming temperature; the duct generally refers to the sub-surface duct, which serves as an "acoustic lens".

\subsubsection{OCEANOGRAPHIC CONDITIONS}

The Beaufort Lens is composed of stratified water masses. Pacific water flows into the Arctic Ocean through the Bering Strait, spreads out across the relatively shallow Chukchi Sea, and enters the Canada Basin. ${ }^{8}$ Pacific Winter Water (PWW) and Pacific Summer Water (PSW) form

\footnotetext{
${ }^{6}$ Toole et al., "Influences of the ocean surface mixed layer and thermohaline stratification on Arctic Sea ice in the central Canada Basin".

${ }^{7}$ Poulsen et al., "Acoustic noise properties in the rapidly changing Arctic Ocean".

${ }^{8}$ Corlett et al., "The Chukchi slope current".
} 
distinct masses due to temperature differences by solar irradiation. ${ }^{9}$ Thus the Beaufort Lens is composed of the following water masses in descending order: ${ }^{10}$

- less saline surface water via ice melt

- warm, saline PSW, which forms the upper boundary of the Beaufort Lens

- colder layer of PWW, which forms the lower boundary of the Beaufort Lens

- warm, saline layer from the Atlantic Ocean

- throughout the rest of the water column, Arctic Deep Water

The Beaufort Gyre, a wind-driven current, is a dominant circulation feature in the Canada Basin. The Beaufort Gyre is the largest Arctic Ocean freshwater reservoir and it has drastically increased its liquid freshwater content over the last two decades. ${ }^{11}$ Fresher surface water ensures a strong halocline that, below the mixed layer depth, preserves ocean layers where temperatures can be up to a few degrees warmer than freezing throughout the year. ${ }^{12}$ The halocline stratification prevents heat fluxes from this warm layer but its accumulation in the ocean interior has consequences for sea ice in the gyre. Recent work by MacKinnon et al. with a variety of fast CTD casts reveal a jet of warm salty water that appears to be subducting beneath a cool fresh surface layer and vertically compressing and breaking up into smaller, spinning eddies; they suggest that the growing heat content in the PSW has a first-order effect on accelerating sea ice melt in the region. ${ }^{13}$

\footnotetext{
${ }^{9}$ Brugler et al., "Seasonal to interannual variability of the Pacific water boundary current in the Beaufort Sea".

${ }^{10}$ Ballard et al., "Temporal and spatial dependence of a yearlong record of sound propagation from the Canada Basin to the Chukchi Shelf"; Duda, "Acoustic signal and noise changes in the Beaufort Sea Pacific Water duct under anticipated future acidification of Arctic Ocean waters".

${ }^{11}$ Zhang et al., "Labrador Sea freshening linked to Beaufort Gyre freshwater release".

${ }^{12}$ Timmermans et al., "Warming of the interior Arctic Ocean linked to sea ice losses at the basin margins".

${ }^{13}$ MacKinnon et al., "A warm jet in a cold ocean".
} 


\subsubsection{Passive acoustics}

One dimension of interest is soundscape and ambient noise analysis. A recent review of an anthropogenic ocean soundscape explains the multi-factorial changes in the Arctic. ${ }^{14}$ Reduced sea ice allows for new species to inhabit warmer waters and compete for "acoustic niche space", changing the biophony. Seger et al. use passive acoustic listeners to suggest that over the last decade, various species of dolphins may be shifting into Arctic waters during years of significant warm water invasion, and that these acoustic detections may anticipate future visual surveys of habitat shifts. ${ }^{15}$

Decreasing sea ice cover also affects the geophony, where periods of ice formation generate higher sound levels. Chen et al. finds that, in comparing ambient noise data between 1994 and 2016, the surface noise is now better modeled by sources at discrete ranges rather than the historical assumption of a continuous and uniform distribution of sources. ${ }^{16}$ A followup study demonstrates how the observed noise vertical directionality is consistent with icemechanical activity along an active pressure ridge roughly $30-50 \mathrm{~km}$ from the recording array, as shown by satellite imagery. ${ }^{17}$

\subsubsection{Active acoustics}

Significant work has gone into the long-term and long-range transmission through the Canada Basin Acoustic Propagation Experiment (CANAPE) from the summer of 2016-17, as the duct has the potential of trapping sound out to significant ranges (greater than $100 \mathrm{~km}$ ) without interacting with ice cover. This study looked at acoustic transmissions on the Chukchi shelf

\footnotetext{
${ }^{14}$ Duarte et al., "The soundscape of the Anthropocene ocean".

${ }^{15}$ Seger et al., "Acoustic documentation of temperate odontocetes in the Bering and Chukchi Seas".

${ }^{16}$ Chen et al., "Spectral, spatial, and temporal characteristics of underwater ambient noise in the Beaufort Sea in 1994 and 2016".

${ }^{17}$ Chen et al., "Temporal and spatial characteristics of the Beaufort Sea ambient noise environment".
} 
into the Canada Basin. During a time period when the warm Pacific Water outflow intersected with the source-receiver track and modified the duct properties,s there was a $20 \mathrm{~dB}$ acoustic energy fluctuation. ${ }^{18}$ This variability was mostly seen over the slope and shelf, with the duct weakening during the winter and spring; the propagation conditions in the deep basin were dominated by the duct. ${ }^{19}$ A year-long analysis of the sound speed fluctuations reiterates the annual persistence of the duct but also indicates that the upper and lower boundaries varied significantly, changing the modal cutoff frequencies by a factor of $2 .^{20}$

Roboticists have leveraged the lower duct to provide acoustic navigation and communication at mesoscale ranges. A long-range acoustic navigation system, consisting of multiple GPS-linked ice buoys with transducers suspended at $100 \mathrm{~m}$ depth, was capable of communications greater than $400 \mathrm{~km}$ with a range accuracy of $40 \mathrm{~m}$ when the sound speed was known. ${ }^{21}$ At $100 \mathrm{~m}$ depth, the source was in a sound speed duct from roughly $50 \mathrm{~m}$ to $200 \mathrm{~m}$.

In 2014, autonomous underwater gliders showed reliable acoustic ranges throughout the water column up to $100 \mathrm{~km}$, but performance was severely degraded the throughput of acoustic packets when the gliders were outside the sound channel. ${ }^{22}$ When gliders were deployed in the CANAPE experiment, receptions from broadband sources were used to position the gliders at the time of reception, using a determinstic estimate for the sound speed and its standard deviation. A post-processing method, which utilized acoustic ray predictions instead of a deterministic estimate, improved range error by a factor of $4 .^{23}$

\footnotetext{
${ }^{18}$ Badiey et al., "Azimuthal and temporal sound fluctuations on the Chukchi continental shelf during the Canada Basin Acoustic Propagation Experiment 2017”.

${ }^{19}$ Ballard et al., "Temporal and spatial dependence of a yearlong record of sound propagation from the Canada Basin to the Chukchi Shelf".

${ }^{20}$ Kucukosmanoglu et al., "Observations of sound-speed fluctuations in the Beaufort Sea from summer 2016 to summer 2017".

${ }^{21}$ Freitag et al., "Long range acoustic communications and navigation in the Arctic".

${ }^{22}$ Webster et al., "Towards real-time under-ice acoustic navigation at mesoscale ranges".

${ }^{23}$ Graupe et al., "Preliminary results for glider localization in the Beaufort Duct using broadband acoustic sources at long range".
} 


\subsubsection{Modeling}

Modeling efforts to explore the coupled environmental and acoustic conditions in the Beaufort Sea have primarily been done by researchers at Woods Hole Oceanographic Institution. One modeling effort projected that, due to ongoing acidification, sound near $900 \mathrm{~Hz}$ could travel up to $38 \%$ further and at ranges of $100-300 \mathrm{~km}$, could be $7 \mathrm{~dB}$ louder. ${ }^{24}$ Motivated by the fixed path time variability in the CANAPE experiment, they present a hydrodynamic model simulation with a horizontal resolution of $500 \mathrm{~m}$ and daily surface forcings to show that the duct may be discontinuous. ${ }^{25}$ Another modeling effort incorporates a sea ice module to produce a time- and space-variable duct. They suggest that ducting is interrupted by the intermittency of the PSW and also show that distribution of modeled ducted conditions remains an area for future improvement. ${ }^{26}$ However, an intercomparison of physically-driven models and observations showed that many models differ in their simulation of the upper ocean circulation in the Arctic. ${ }^{27}$

Taking a step out to climate models, several CMIP4 models indicate ice free summers by $2037,{ }^{28}$ but sea ice cover is not a high accuracy feature across more advanced CMIP5 modeling efforts. ${ }^{29}$ Furthermore, the Beaufort Lens is not represented in some of the best performing sea ice climate models-Max Planck Institute Earth System Model and the Norwegian Earth System Model-most likely due to the coarse spatial resolution hindering Pacific water transport through the Bering Strait.

\footnotetext{
${ }^{24}$ Duda, "Acoustic signal and noise changes in the Beaufort Sea Pacific Water duct under anticipated future acidification of Arctic Ocean waters".

${ }^{25}$ Duda et al., "Long-range sound propagation in the Canada Basin".

${ }^{26}$ Duda et al., "Effects of Pacific Summer Water layer variations and ice cover on Beaufort Sea underwater sound ducting".

${ }^{27}$ Aksenov et al., "Arctic pathways of Pacific Water".

${ }^{28}$ Wang et al., "A sea ice free summer Arctic within 30 years?"

${ }^{29} \mathrm{Wu}$ et al., "Assessment of Arctic sea ice simulations in CMIP5 models".
} 


\subsection{Duct STRENGTH PARAMETER}

The geometrical duct parameter, $P\left[\mathrm{~m}^{2} / \mathrm{s}\right]$, quantifies duct strength. ${ }^{30}$ Given a local maximum in the sound speed profile as a duct top at $z_{t}$,

$$
\begin{aligned}
P & =\Delta c \Delta z \\
\Delta c & =c\left(z_{t}\right)-c_{\min } \mid \text { duct } \\
\Delta z & =\text { distance from the duct top where } c \text { rises again to that maximum value }
\end{aligned}
$$

Because $P$ uses the minimum and maximum sound speed values, it is not sensitive to the shape of the duct itself-a rectangular duct may have the same duct strength as a triangular one. This thesis introduces the integral parameter, $x$, for the duct strength, where $x$ is defined as the area between the top and bottom of the duct with respect to the SSP. Given that $z_{b}$ is the depth at which the sound speed rises to the same value as that of $z_{t}$,

$$
\chi=\int_{z_{b}}^{z_{t}} c(z) d z
$$

Figure 3.3 shows one instance of $P$ and $x$ for the nominal sound speed during ICEX16. The top of the duct, which makes a feature that looks like a knee or a tongue, is found algorithmically through a local maximum search with mnimium constraints on prominence and distance between local maxima. Then, the first corresponding depth at which the sound speed equals the sound speed at the knee is found, and used to set the integration bounds for the duct strength. If multiple ducts are found, only the metrics for the largest duct are saved. Naturally, this ignores any micro-ducts that might exist within the largest duct. From the ITP dataset of

\footnotetext{
${ }^{30}$ Duda et al., "Long-range sound propagation in the Canada Basin".
} 


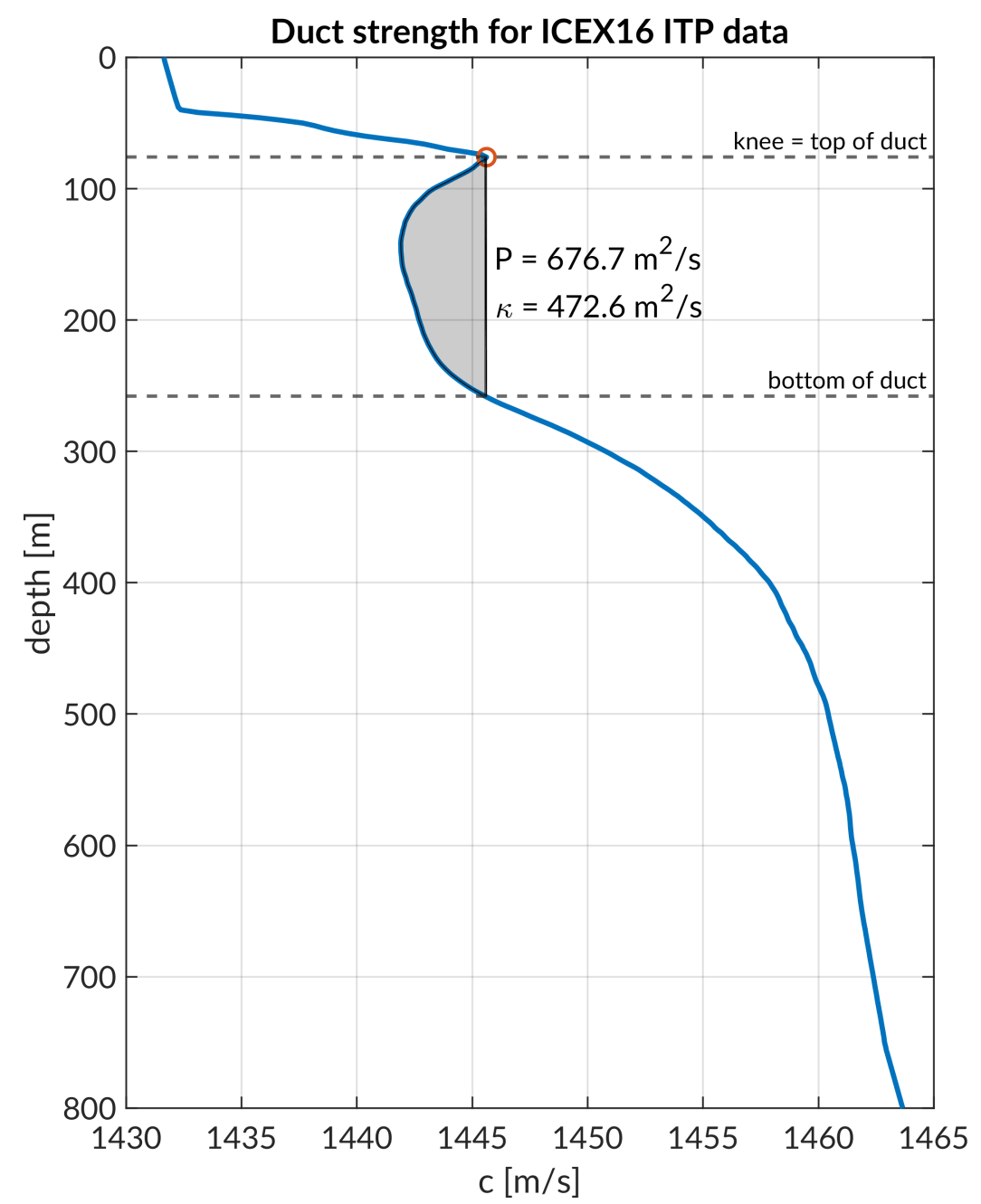

Figure 3.3: A visual explanation of the duct strength parameter. $P$ refers to the duct strength parameter introduced by Duda et al.; $x$ refers to the shaded region, a more conservative integral parameter introduced in this thesis.

74,683 profiles in the Beaufort Sea from 2006 to 2020, slightly less than half $(30,465)$ satisfied the sampling criteria (valid data below $40 \mathrm{~m}$ and above $300 \mathrm{~m}$ ) to ascertain duct strength.

Intrinsically, $P$ will always be greater than $x$, so care is required when setting thresholds for exploitable ducts. Duda et al. indicate a weak duct of $P=150 \mathrm{~m}^{2} / \mathrm{s}$ where roughly $30 \%$ of the samples from an MITgcm primitive equation model for one year have no duct. Similar statistics can be ascertained for the ITP dataset by first comparing $P$ and $x$ for each ITP profiler. Figure 3.4 determines a scaling factor of 0.61 between the two metrics, which indicates 
a weak duct of $x=90 \mathrm{~m}^{2} / \mathrm{s}$. Compared to model-derived distribution, the data-driven duct distribution narrows the region of log-normal behavior from $0.25-0.9$ to $0.4-0.7$. The data exhibits a greater tendency towards weak ducts, corroborating the historical Arctic profile of a monotonically increasing sound speed; and roughly 50\% of ITP profiles have a duct strength less than $x=90 \mathrm{~m}^{2} / \mathrm{s}$. At the same time, and unsurprisingly, the data also contains ducts that are far stronger than those developed in modeling efforts.

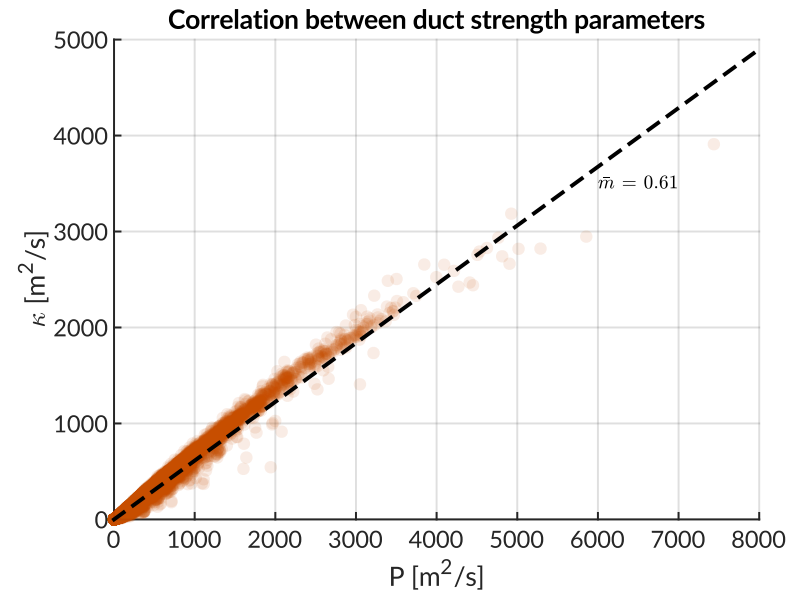

(a) A scatter plot to see the scaling of the two duct metrics as introduced in figure 3.3 .

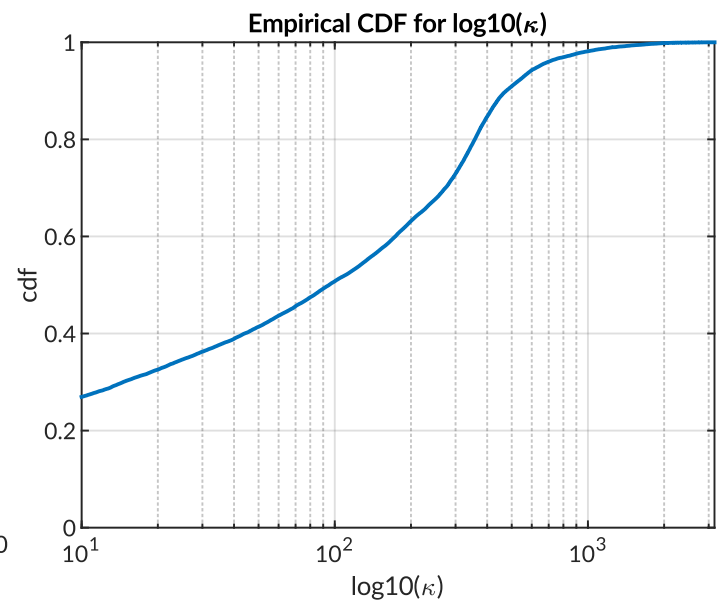

(b) A lognormal cumulative distribution function of the duct strength, $x$.

Figure 3.4: The equivalent weak duct strength is where $x<=90 \mathrm{~m}^{2} / \mathrm{s}$, as shown on the left. On the right, the duct strength is presented in $\log 10$. Roughly half of all ITP observations are characterized as less than a weak duct.

Figure 3.5 shows a heatmap of sound speed from the ITP dataset for varying duct strength bins. These bins were chosen by multiplicative factors of the weak duct strength. The classical Arctic SSP is fairly well represented when $x<=90 \mathrm{~m}^{2} / \mathrm{s}$. The darker region shows a monotonically increasing SSP. Duct strength where $x>90 \mathrm{~m}^{2} / \mathrm{s}$ increases the sound speed observed at the knee without dramatically changing the depth of the knee or the sound speed structure below 150 meters. The PSW is an integral factor in driving the ducted propagation environment; spatio-temporal variability in the PSW would align with variability of any acoustic metric such as transmission loss. When $x>270 \mathrm{~m}^{2} / s$, the region between 200 and 
$300 \mathrm{~m}$ has two distinct threads; the lower thread would contribute to a stronger duct. The dual threaded nature and the relative spreading of the sound speed from 200 to $300 \mathrm{~m}$ compared to greater depths may indicate the mixing between PWW and Atlantic Water. This mixing is most prominent when $x \leq 90 \mathrm{~m}^{2} / \mathrm{s}$, where it extends up to $100 \mathrm{~m}$. Of course, the lack of stratification defines the historical Arctic profile, where the sound speed of the first $300 \mathrm{~m}$ is driven by co-increasing temperature and salinity.
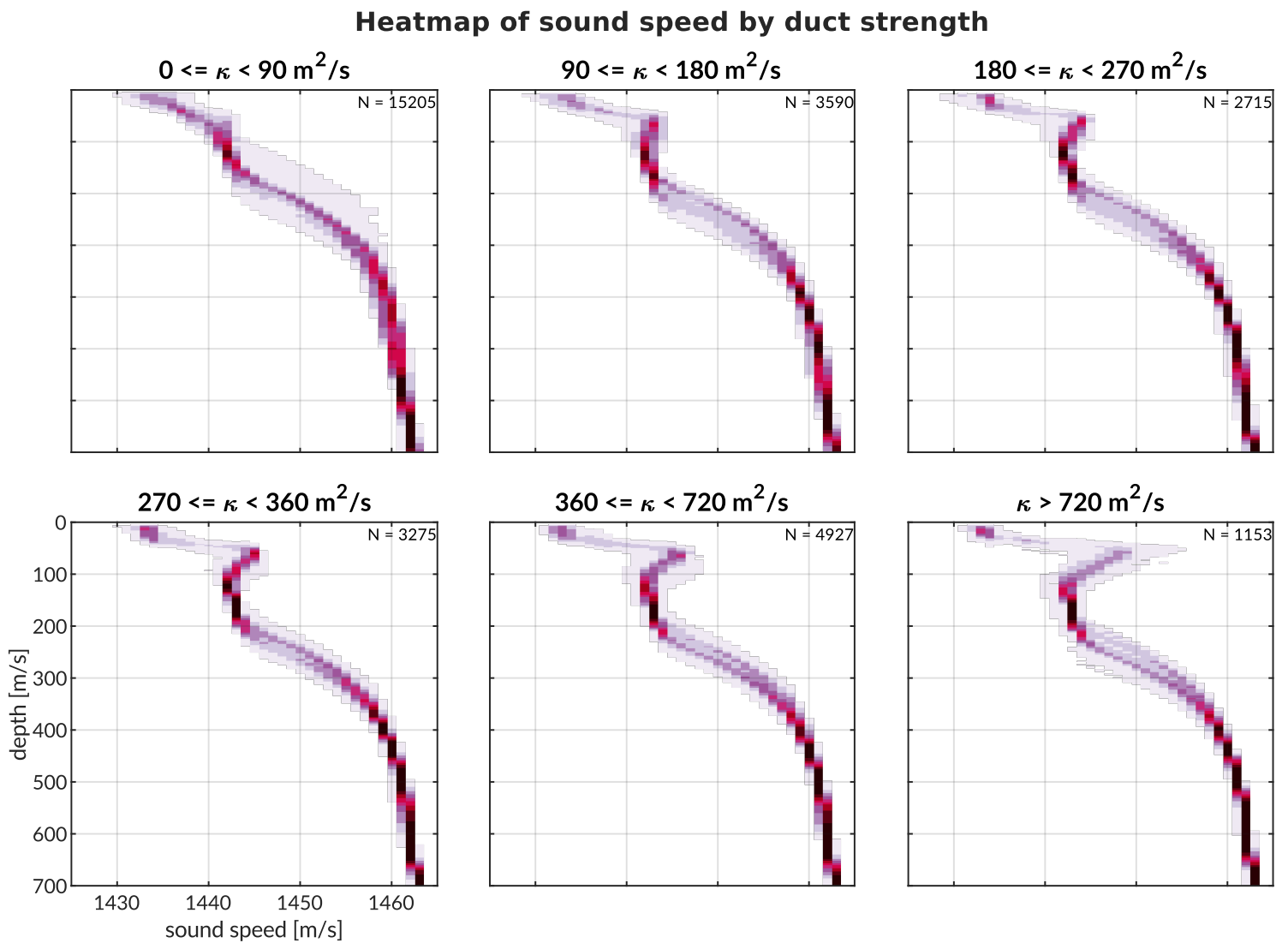

Figure 3.5: A heat map of sound speed profile distribution sorted by duct strength. The darker color indicates a more commonly found value from the ITP dataset. Lighter colors show the variance of the sound speed profile, which is most seen in the upper 300 meters.

It is important to note what this sub-surface duct parameter captures and what it does not. By finding the knee and the depth at which the sound speed equals that of the knee, it captures the boundaries of the shadow zone and is an easy visualization for lens. But, this empirical cal- 
culation has little intuitive connection to acoustic propagation-or the governing physics-on its own and it completely ignores the absolute depth at which the duct presides. The geometrical nature means an extremely thin and strong duct might have the same strength as a broad but weak duct; thankfully, the duct shape is not random and this equivalence happens rarely, if ever.

\subsection{Temporal analysis}

This section introduces the temporal characteristics of ducted conditions in the Beaufort Gyre. Here we show three metrics for the duct: the duct strength, the temperature difference in the duct, and the knee height. Temperature difference, as opposed to sound speed difference, is more intuitive to understand warming and is the main driver behind sound speed difference in the duct. There is much stronger interannual than seasonal variability for all three metrics. The duct strength and temperature difference is shown for all valid observations while the knee depth is only shown for $x \geq 90$.

Figure 3.6a shows $x$ across the 16 -year time period with the mean, median, $25^{\text {th }}$ and $75^{\text {th }}$ percentile superimposed. From 2006 to 2012, the median and mean duct strength are 1-2x a weak duct. Events in 2007 and 2010-2011 show anomalously strong ducts. From 2013 onward, there is a notable lack of unexploitable ducts compared to previous years, and the mean and median duct strength are 2-4x a weak duct. In contrast, only 2019 shows a similar frequency of unexploitable ducts as compared to 2006-2012. Curiously, the year 2020 has seen unprecedented high duct strength compared to previous years except for 2010-2011. This recent ballooning of duct strength in 2020 is not explained by the spatial distribution of the profilers compared to that of all others years. 
The temperature difference, $\delta t$, in the duct has not been directly studied. Figure 3.7 a shows the 16-year timeseries of the temperature difference, where the mean and median create an increasing step-like pattern. From 2006-2009, $\delta t$ centers around 0.5 C; from 2010-2012, 1.0 C; from 2013 to $2019,1.5$ C; and in 2020, roughly 2.5 C. This framing points towards the warming Arctic at subsurface depths and strongly correlates with the increase in ducted conditions over the past 16 years. Most notably, the $\delta t$ over 2020 show close to no overlap with that of 2006-2010, in a similar vein to the duct strength.

The knee depth was characterized as being between $60-80 \mathrm{~m}$ based on oceanographic data taken during ICEX16. ${ }^{31}$ Figure 3.8a shows the 16-year timeseries of the knee depth, which shows a depth variability of $40-100 \mathrm{~m}$, though it is most concentrated around $60 \mathrm{~m}$. Any given year sees a knee depth spread of around 30-40 m, with some years, like 2008-09, or 2018-2019, seeing much wider spreads. These findings are plagued by sparse and intermittent data, especially from 2008-09 and in 2016. The anomalous duct strength seen in 2020 is not correlated with an anomalous knee height, which corresponds to further stratification between the PSW and PWW.

There is a weak monthly cycle, shown in figure 3.6b, where the mean duct strength fluctuates from $180-280 \mathrm{~m}^{2} / \mathrm{s}$ and the median duct strength fluctuates from $70-140 \mathrm{~m}^{2} / \mathrm{s}$. It is strongest in November and December, and weakest in February through May. A closer look at the spread of individual observations may indicate a bimodal distribution of ducts stronger than the median by roughly $200 \mathrm{~m}^{2} / \mathrm{s}$ throughout the year. This bimodal nature is corroborated by the monthly temperature difference in ducts, in figure $3.7 \mathrm{~b}$. While the mean and median temperature difference are closely linked, overall the increase in November and December is only $0.2 \mathrm{C}$ than the average throughout February through May.

\footnotetext{
${ }^{31}$ Poulsen et al., "Acoustic noise properties in the rapidly changing Arctic Ocean".
} 
The weak monthly cycle does not carry over to knee depth (figure 3.8b). Even though the ITPs fail to resolve the very shallow and warm mixed layer characteristics in summertime, ${ }^{32}$ they are accurately sampling warming at the knee and duct depths. The weak monthly duct strength cycle is more likely driven by warming than knee depth movement.

${ }^{32}$ Toole et al., "Influences of the ocean surface mixed layer and thermohaline stratification on Arctic Sea ice in the central Canada Basin". 


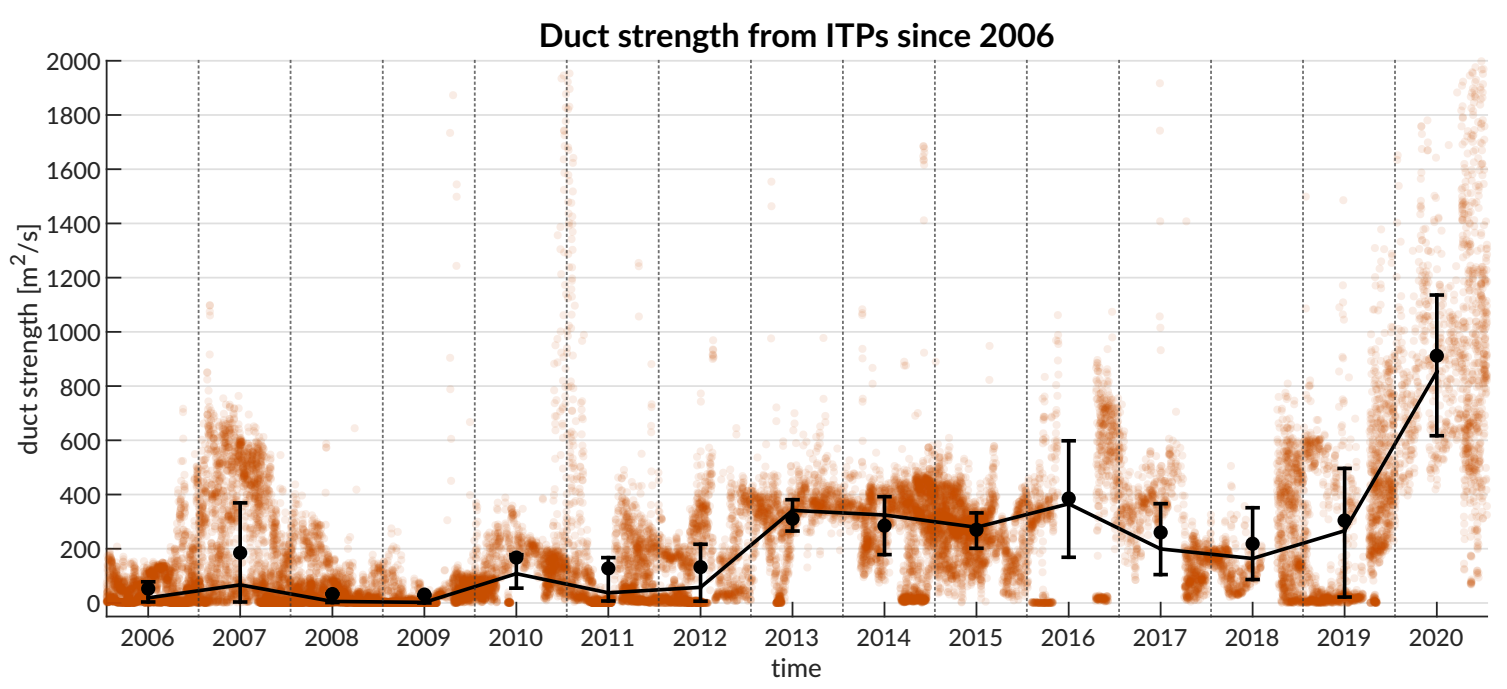

(a) Each orange dot indicates the duct strength from an individual ITP profile with respect to the full time-series. The black line across years connects the median value; the error bars show the $25^{\text {th }}$ and $75^{\text {th }}$ percentile; the black circle indicates the mean.

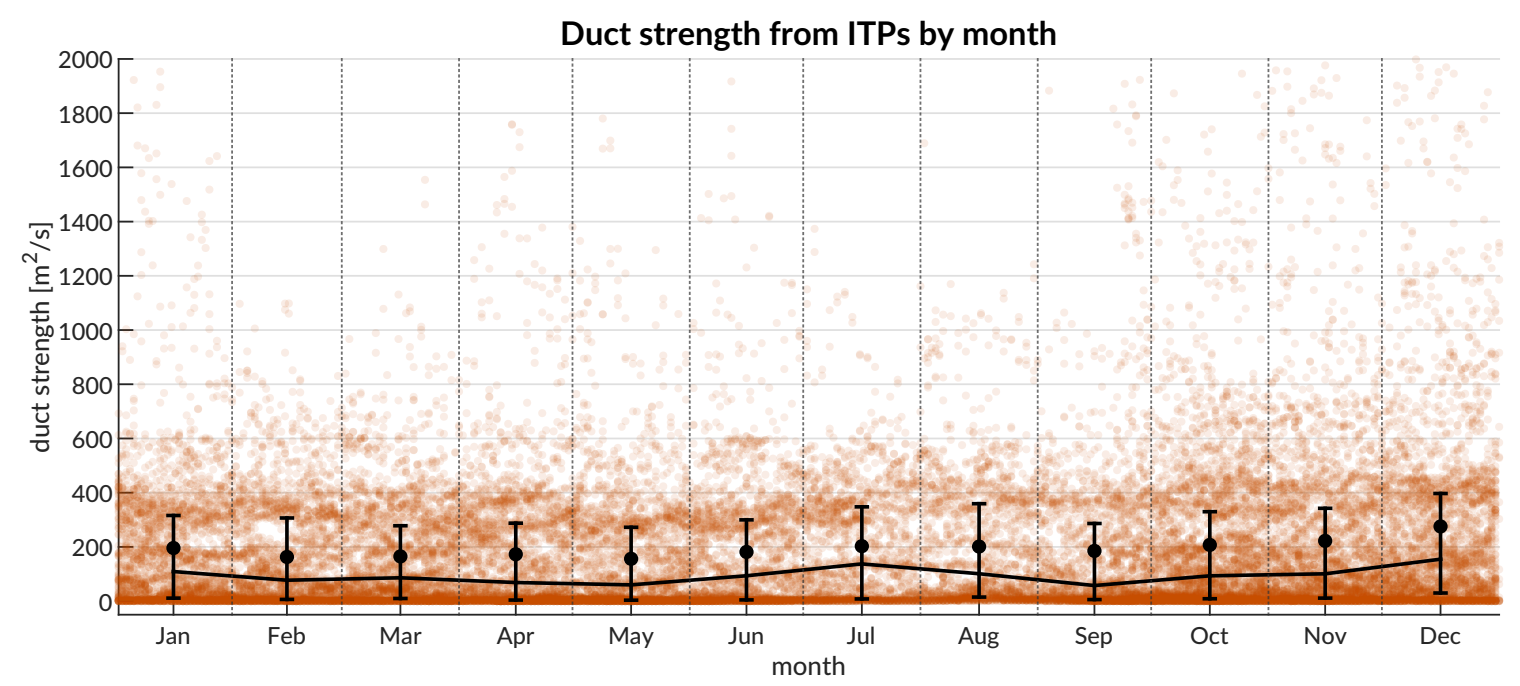

(b) Each orange dot indicates a duct strength calculation from an individual ITP profile with respect to the month and day. The black line across months connects the median value; the error bars show the $25^{\text {th }}$ and $75^{\text {th }}$ percentile; the black circle indicates the mean.

Figure 3.6: The duct strength trends, annually and monthly, from the ITP dataset for the Beaufort Gyre. Figure 3.6a shows a notable increase in the duct strength since 2019, which only matches an event in the winter of 2010-11. Figure 3.6b shows the strong presence of profiles with no ducts, roughly $25 \%$ of the dataset, and no dominant seasonal trends in duct strength. 


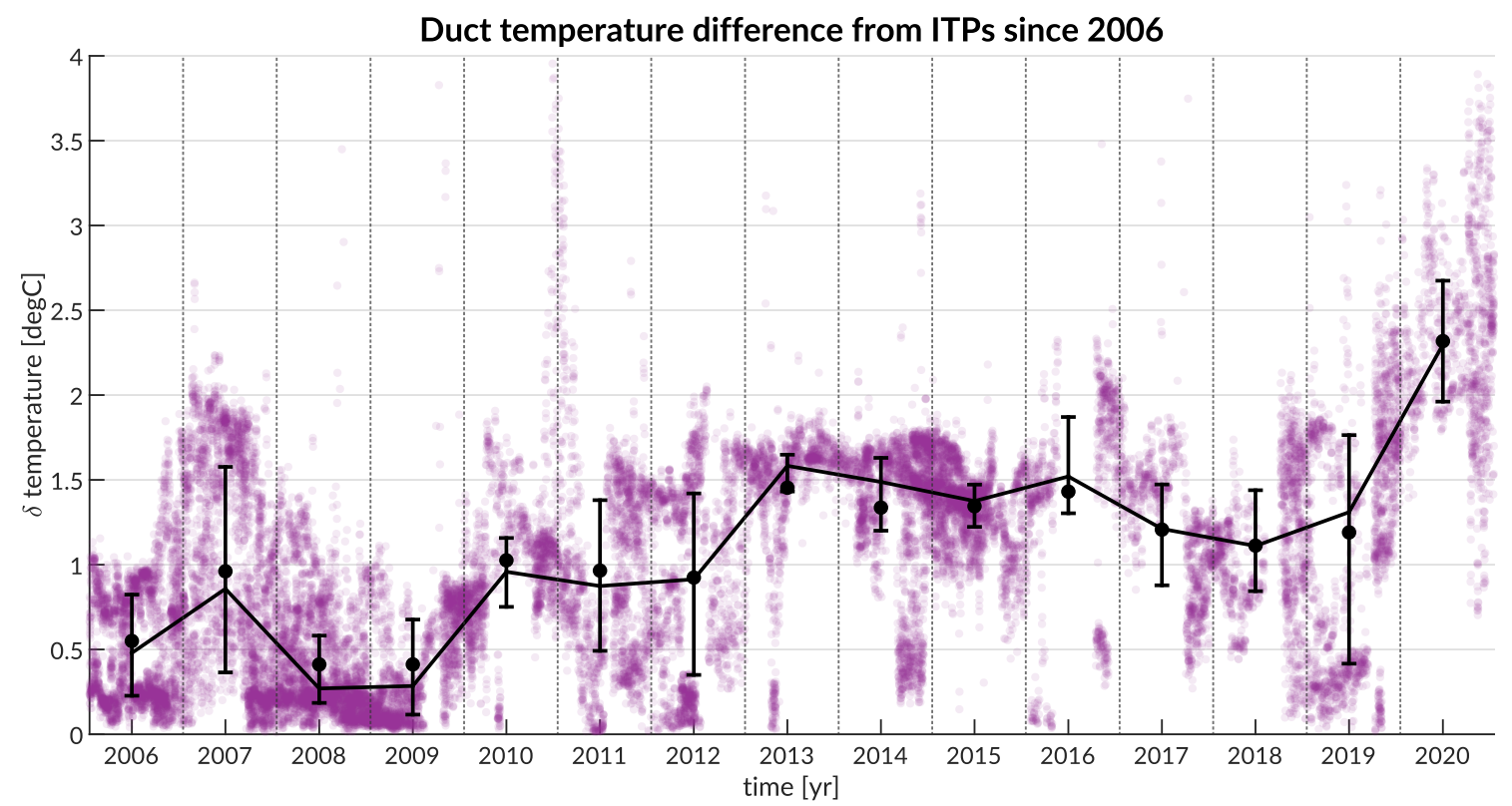

(a) Each purple dot indicates the temperature difference inside the duct from an individual ITP profile with respect to the full time-series. The black line across years connects the median value; the error bars show the $25^{\text {th }}$ and $75^{\text {th }}$ percentile; the black circle indicates the mean.

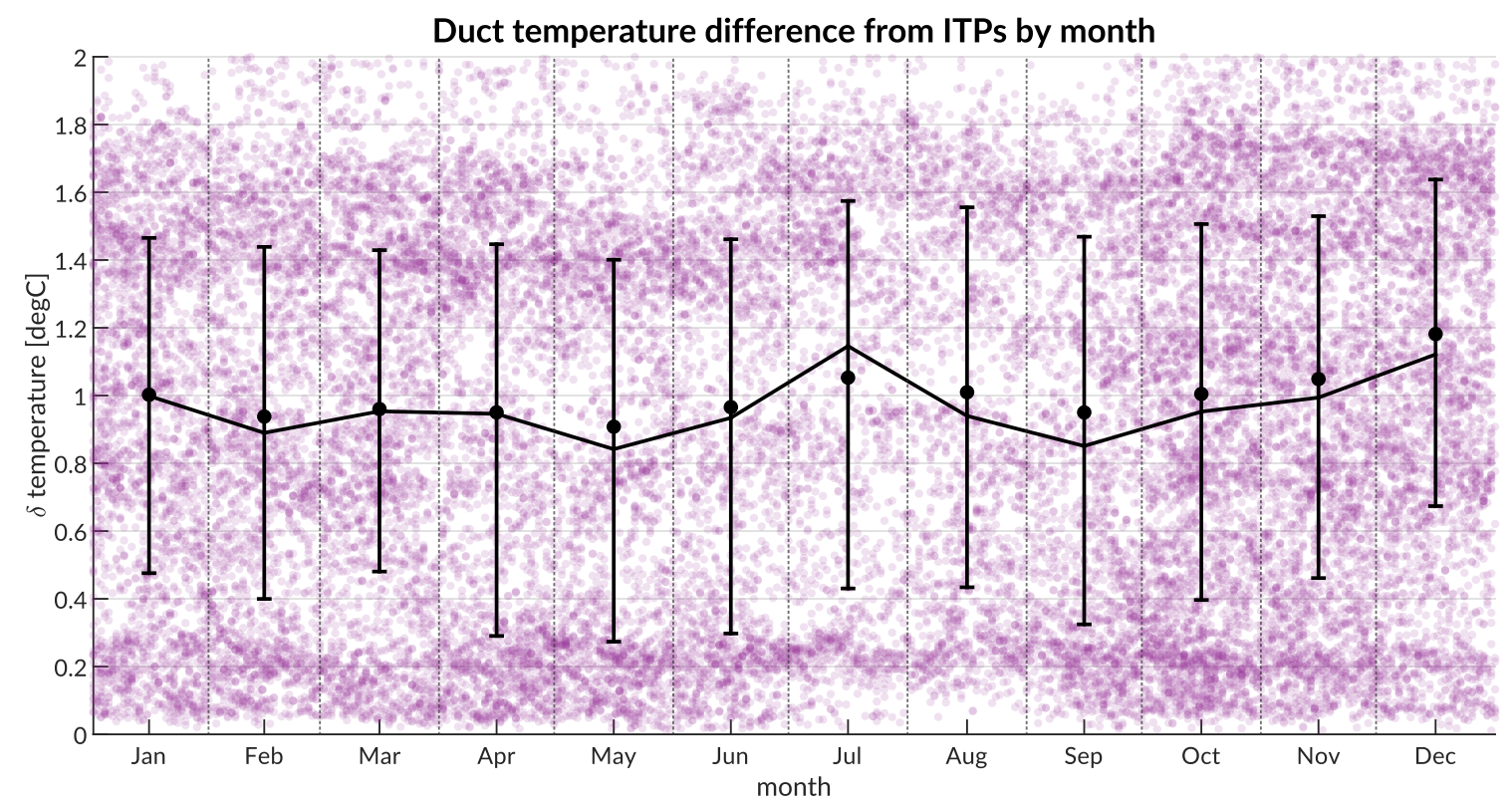

(b) Each purple dot indicates the temperature difference inside the duct from an individual ITP profile with respect to the month and day. The black line across months connects the median value; the error bars show the $25^{\text {th }}$ and $75^{\text {th }}$ percentile; the black circle indicates the mean.

Figure 3.7: The temperature difference trends, annually and monthly, from the ITP dataset for the Beaufort Lens. Figure 3.6a shows a notable increase in the temperature difference since 2019 , which only matches an event in the winter of 2010-11. Figure 3.6b shows the strong presence of profiles with minimal temperature, roughly $25 \%$ of the dataset, and no dominant seasonal trends in duct strength. 


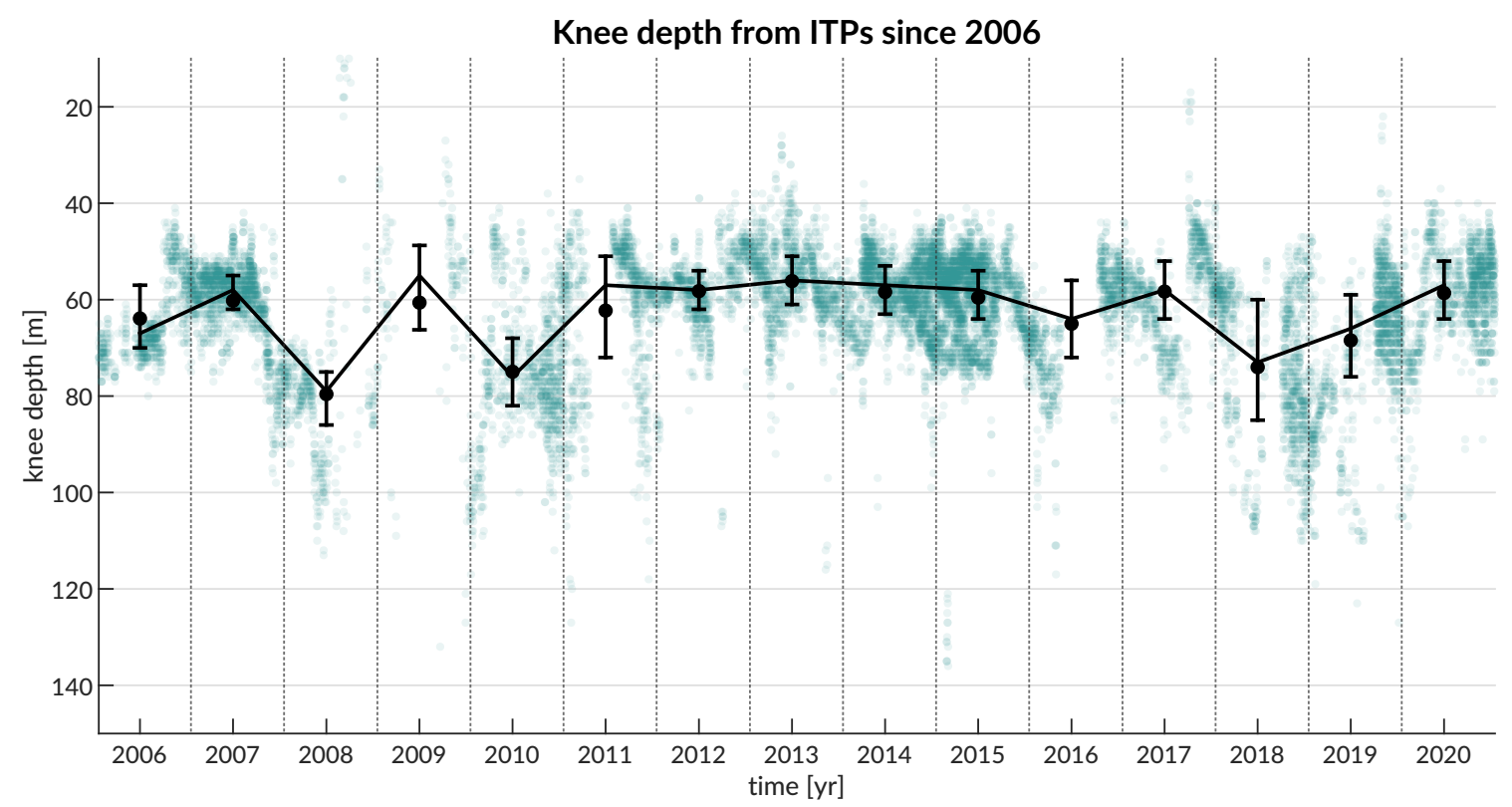

(a) Each teal dot indicates the knee height for the strongest duct from an individual ITP profile with respect to the full time-series. The black line across years connects the median value; the error bars show the $25^{\text {th }}$ and $75^{\text {th }}$ percentile; the black circle indicates the mean.

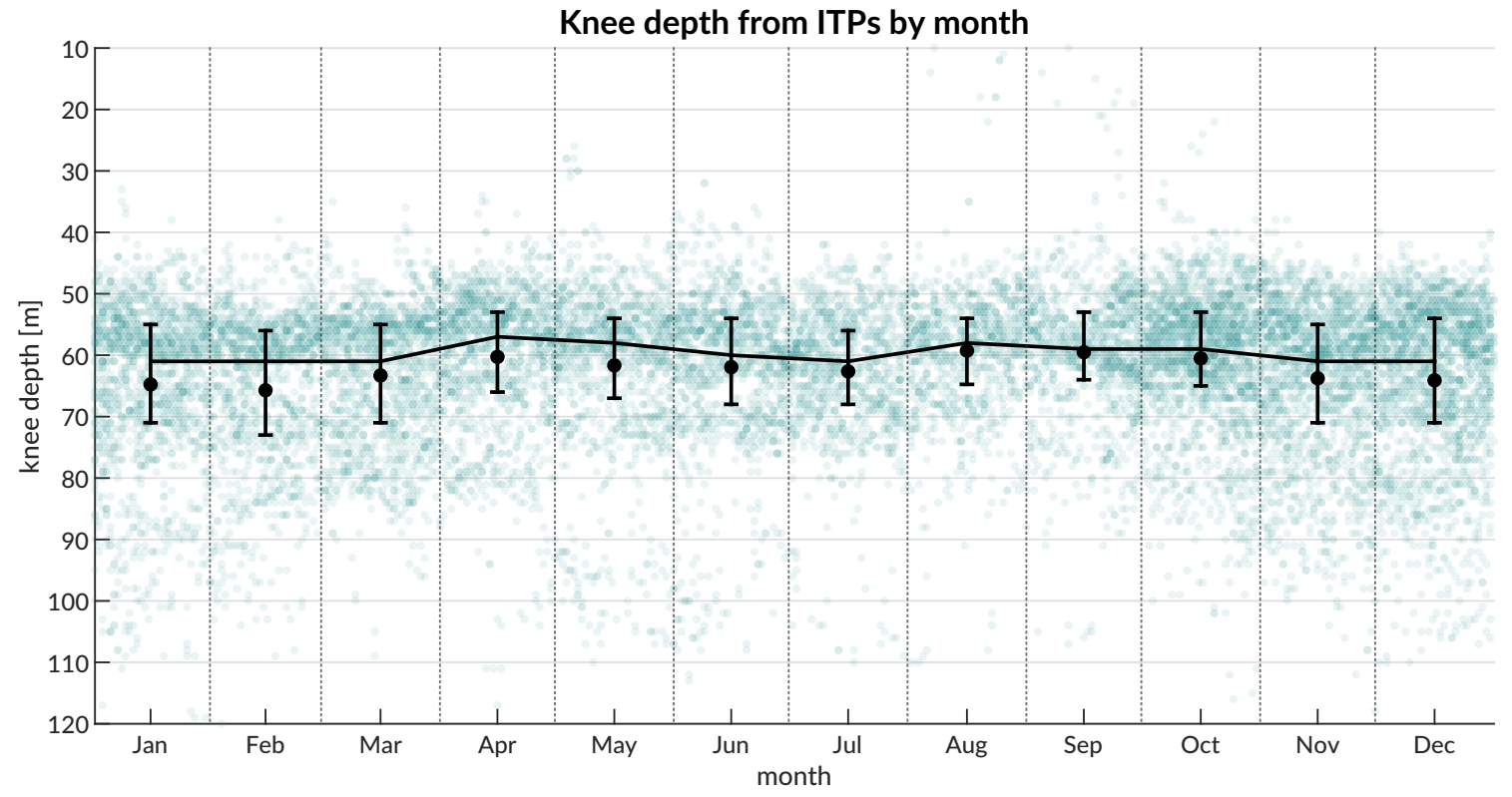

(b) Each teal dot indicates the knee height for the strongest duct from an individual ITP profile with respect to the month and day. The black line across months connects the median value; the error bars show the $25^{\text {th }}$ and $75^{\text {th }}$ percentile; the black circle indicates the mean.

Figure 3.8: The knee height trends, annually and monthly, from the ITP dataset for the Beaufort Gyre. Figure 3.8 a shows variance of the median knee height between 55 to 80 meters. Figure 3.8b shows no dominant seasonal cycle, as the median knee depth stays between 55 to 60 meters. 


\subsection{SPATIAL ANALYSIS}

While spatial analysis in oceanography defaults to horizontal, depth dependence is the key dimension for acoustic propagation. This section looks at duct strength vertically and laterally. Because of the sparse data conditions and extreme tails in the duct strength distribution, the median is a more robust indicator than the mean. The results presented in this section, to my knowledge, are the first data-driven mapping of the Beaufort Lens.

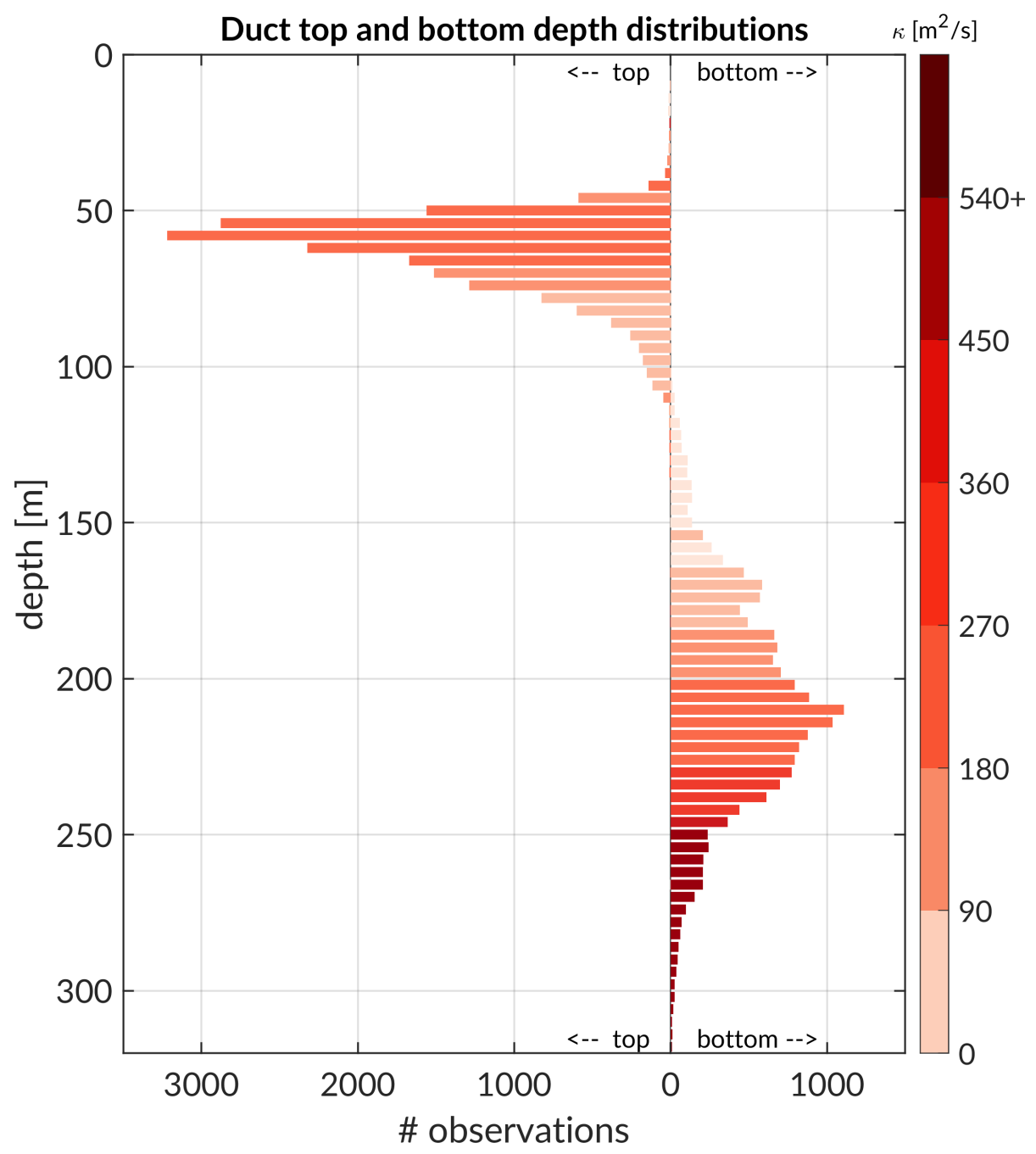

Figure 3.9: A side-by-side probability distribution for the duct top and bottom depths, on the left and right, respectively. Each bin is 4 meters wide and is colored by the median duct strength at that depth, where darker means stronger. 
Figure 3.9 shows the count distribution of the observed top and bottom of duct depths where each bar is colored by duct strength. The data shown here carries over for all valid ITP observations from figure 3.6. The top of the duct is seen to be most prominently between 52-62 m, a slightly narrower and shallower range than observed in $2016 .{ }^{33}$ The median duct strength decreases as the top of the duct goes further away from the surface (or mixed layer). Notably there are very few knees above $40 \mathrm{~m}$, and the deepest observed duct top and shallowest observed duct bottom are just over $100 \mathrm{~m}$. The separation between these two metrics further corroborates the intense stratification of the Beaufort Lens.

The bottom of the duct is most likely to be observed around $210-220 \mathrm{~m}$. Intuitively, the strength of the duct increases as the bottom of the duct increases. The strongest ducts reach down to roughly $300 \mathrm{~m}$ and unexploitable ducts tend to bottom out from 120-160 m. This framing provides new guideposts for the Beaufort Lens sub-surface duct.

Pivoting to horizontal variability, figure 3.10 is a map of the median strength of the Beaufort Lens across the gyre. The discrepancies in sampling density are represented through the size of the circles. The light pink circles show where the median duct strength consists of unexploitable ducts. This region, which indicates the classical Arctic sound speed profile, dominates the northern part of the Beaufort Gyre. It is also present in the shelf, though there is a considerable lack of data in this region, and is interspersed between regions of stronger duct strengths. The patchiness, especially in the diagonal from $78 \mathrm{~N}, 155 \mathrm{~W}$ to $75 \mathrm{~N}, 130 \mathrm{~W}$, corroborate earlier conclusions from modeling that the duct is not necessarily continuous. ${ }^{34}$

The duct seems to be fairly widespread throughout the lower part of the gyre. North of Camp Sargo shows exploitable ducts with varying strength and many are 2-3x a weak duct. The darkest regions indicate extremely strong ducts, which seem to be scattered through-

\footnotetext{
${ }^{33}$ Poulsen et al., "Acoustic noise properties in the rapidly changing Arctic Ocean".

${ }^{34}$ Duda et al., "Long-range sound propagation in the Canada Basin".
} 


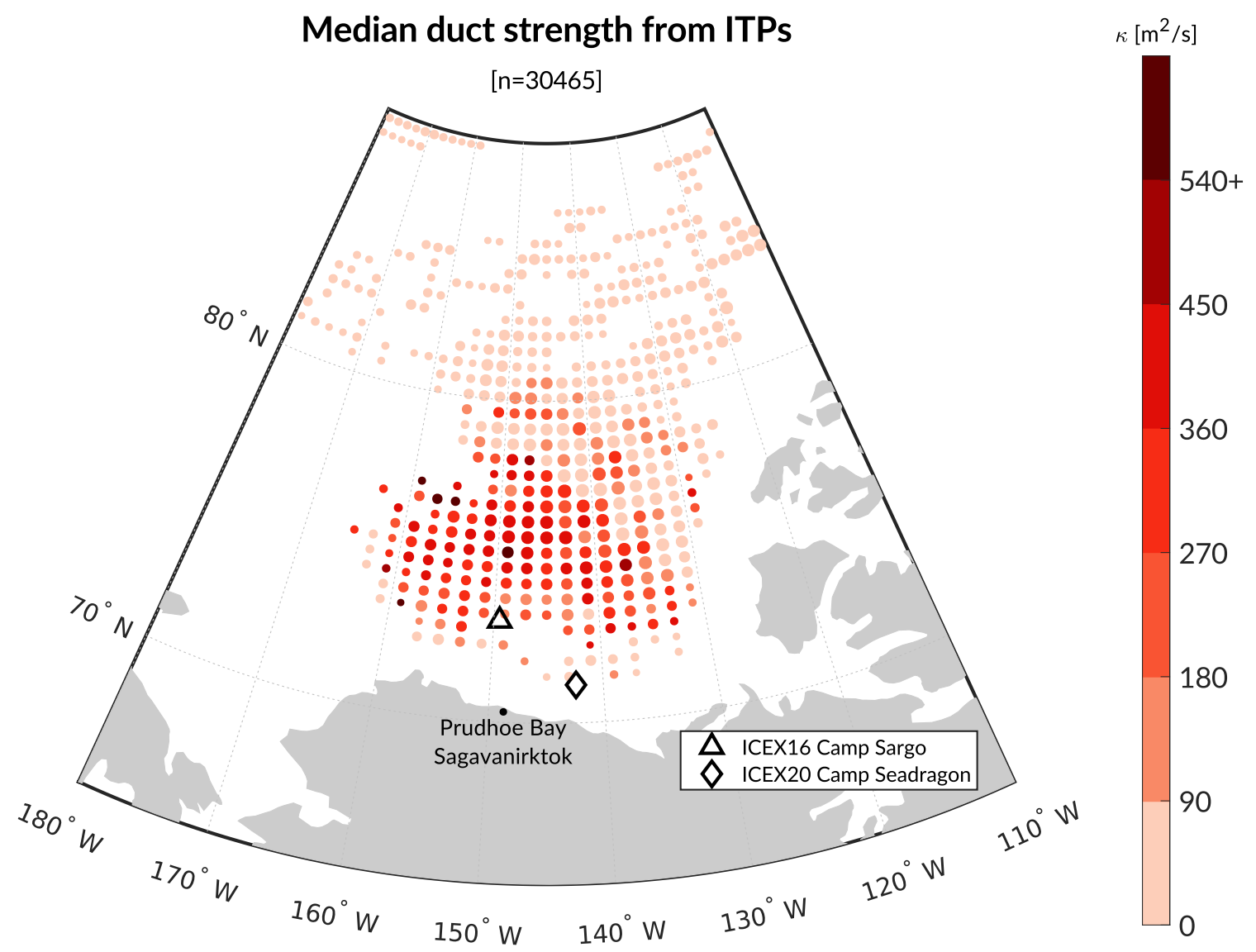

Figure 3.10: A map of the median duct strength across the Beaufort Gyre. Each dot represents a bin of 2 degrees longitude by 0.5 degrees latitude, where its size reflects the density of observations as shown by figure 3.2. The darker color represents a stronger duct.

out the gyre. Some patchiness may be due to inherent (and unsolvable) mismatches in temporal sampling density, as a map of the maximum duct strength would have many observations where $x>540 \mathrm{~m}^{2} / \mathrm{s}$. Most importantly, this mapping highlights the need for continued sampling and acoustic studies in the Beaufort Lens, as its dynamics are an easily measureable harbinger of change in the Arctic. 


\subsection{SuRfaCE LAYER DEPTH}

Studies that use ITP data to examine the mixing or the heat content of the Beaufort Gyre employ standard physical oceanography parameters that have relevance to the Beaufort Lens. Toole et al. show that, in the winter, the mixed layer depth averaged $24 \mathrm{~m}$ and rarely exceeded $40 \mathrm{~m}$; in the summer, it averaged just $16 \mathrm{~m}$ but is likely a biased estimate due to the profiling mechanism. They use a $0.01 \mathrm{~kg}^{3} / \mathrm{m}$ density criterion to calculate the mixed layer depth. ${ }^{35} \mathrm{~A}$ follow up study distinguishes between the homogeneous mixed layer and the surface layer by using a less strict $0.25 \mathrm{~kg}^{3} / \mathrm{m}$ criterion. The surface layer marks the clear barrier of the strong halocline that impedes vertical heat flux. For the Beaufort Lens, this effectively places an upper bound on where the knee would reside.

The top row in figure 3.11 plots a normalized two-dimensional density histogram of surface layer depth and knee depth across three bounds of duct strengths (unexploitable, mild, strong). Across each of these categories, the domain of both parameters is consistent $-10-55 \mathrm{~m}$ for the surface layer depth and 40-100 $\mathrm{m}$ for the knee depth. For unexploitable ducts, the "knee" detected algorithmically, though trivial, is most often found between 70-80 m with surface layer depths of 20-35 m. This is in stark contrast to the knee and surface layer depths of mild and strong ducts. Both of those joint distributions show highest frequency for knee depths of 50-60 $\mathrm{m}$ and surface layer depths of $25-45 \mathrm{~m}$. The surface layer and knee depth distribution is more uniform for strong ducts compared to mild ducts. Given the intense stratification of the Beaufort Lens, this finding may explain that the lens is discontinuous because it is in stages of evolution, as eddies and filaments strengthen and weaken the duct. ${ }^{36}$

\footnotetext{
${ }^{35}$ Toole et al., "Influences of the ocean surface mixed layer and thermohaline stratification on Arctic Sea ice in the central Canada Basin".

${ }^{36}$ Duda et al., "Long-range sound propagation in the Canada Basin".
} 
The bottom row in figure 3.11 shows an empirical probability distribution of the difference between the surface layer and knee depths. Unsurprisingly, this is rarely negative. And intuitively, the peaks for each depth distribution differ across unexploitable, weak, and strong ducts. The unexploitable duct distribution tends to be the largest, with a maximum at 40$45 \mathrm{~m}$. The strong duct distribution has a maximum around $25-35 \mathrm{~m}$, with the majority of the data between $10-40 \mathrm{~m}$. The weak duct distribution is fairly uniform from $15-55 \mathrm{~m}$, which may further suggest a dynamic evolution between differing duct strengths. Given that the data is taken over several years and throughout the gyre, the differences in duct strength mapping onto surface layer depth and knee depth likely represent different proportions and avenues of the Pacific water masses. The nature of the mixed and surface layers are incredibly important for short-range vehicle operations, as they determine the lower bound for the surface sound channel. 
Mapping knee height vs surface layer depth for varying duct strength, $\kappa$
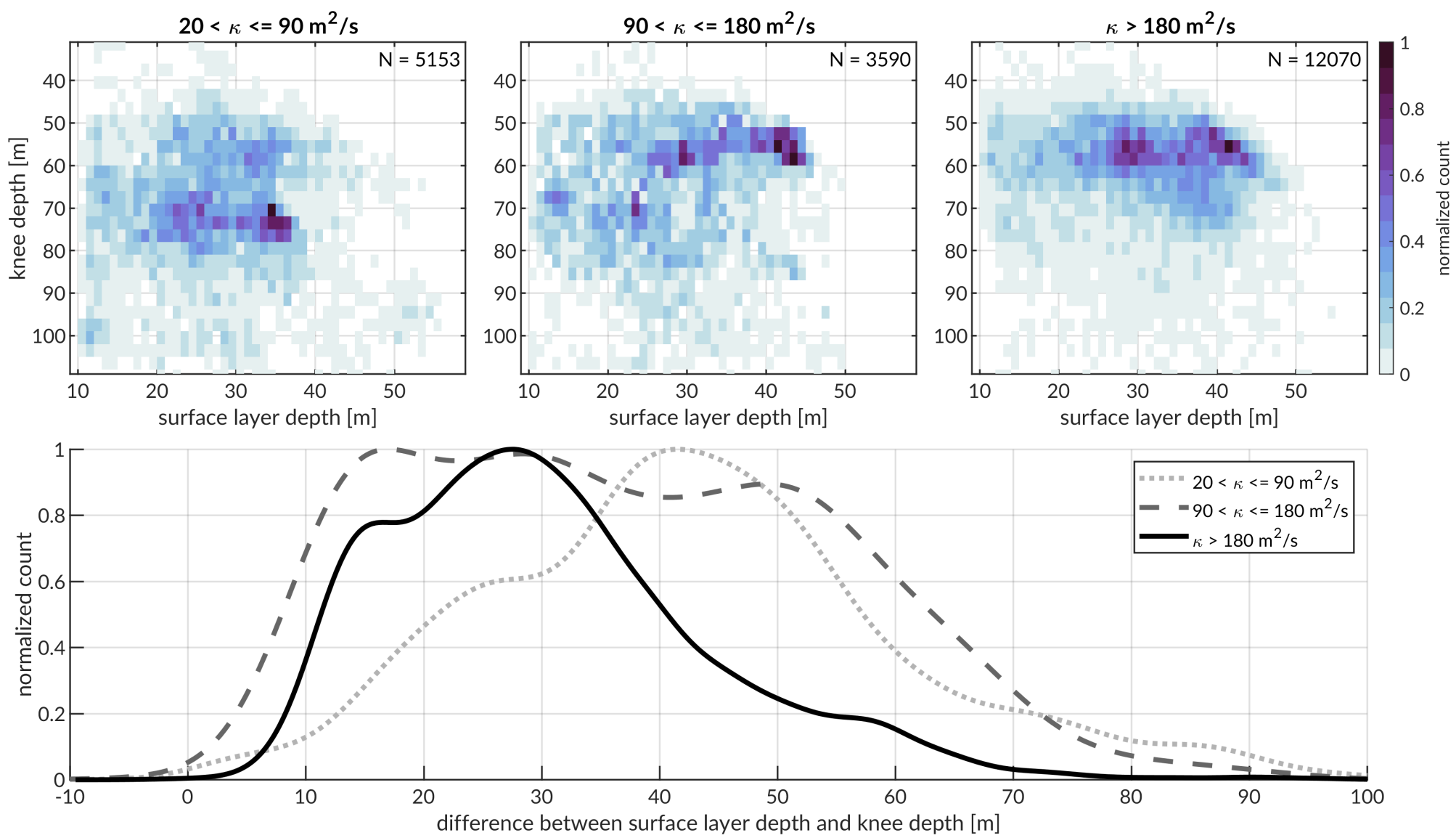

Figure 3.11: The top row shows heat maps of knee height versus surface layer depth, grouped for consecutive spans of duct strength. The $\mathrm{x}$-axis shows surface layer depth and the $\mathrm{y}$-axis shows knee depth. The normalized count scales the color by the maximum value found in each grouping. On the plot all the way to the left, the bounds for $x$ begin at 20 , not 0 , to remove any unexploitable ducts and/or those conjured by sensor noise. The bottom row shows a normalized kernel density estimate of the difference between the surface layer depth and the knee depth for consecutive spans of duct strength. 


\subsection{COMPARISON TO HYCOM \& HISTORICAL DATA}

The obvious challenge to understand the Beaufort Lens-and its evolution-is the lack of dense spatio-temporal data. The other challenge is that this feature is unaccounted for in historical databases and HYCOM models. These two sources reflect the information available onboard submarines which play a direct role in the submarine's decisions regarding where and how to employ its own sensors. ${ }^{37}$

Figure 3.12 is a representative comparison of historical, HYCOM, and ITP data for ICEX16 conditions. An exhaustive comparison, say, one that compares every single ITP observation with its HYCOM counterpart, is needlessly difficult in aggregating the model from global servers. However, it might also be trivial-initial comparisons of HYCOM reanalysis with ITP observations for March of 2016 through 2020 show no ducts in the reanalysis to begin with.

Salinity below $100 \mathrm{~m}$ is well captured by HYCOM and historical data. Neither input resembles the halocline seen in the ITP data nor do they consider the impact of freshwater melt. HYCOM captures the well-mixed nature of the upper $50 \mathrm{~m}$ whereas the historical database shows a positive gradient. Even though this discrepancy is obvious, it has little impact on the shape of the sound speed at the surface, just its value.

Mismatches in temperature are the main driver for mismatches in sound speed, due to it playing a more dominant role in determining sound speed and being more incorrect compared to the ICEX16 data. Both HYCOM and the historical profile show a temperature inversion, with colder water on top of warmer water, but do not preserve the observed thermoclines. The historical profile drastically underestimates the maximum temperature observed at $75 \mathrm{~m}$ but preserves

\footnotetext{
${ }^{37}$ Personal conversations with Lieutenant Commander Dan Goodwin
} 
how the colder PWW would create a temperature (and sound speed) minimum. HYCOM better resolves the warmer water mass near $70 \mathrm{~m}$ but does not resolve the colder water mass below it.

Neither the historical database nor the HYCOM reanalysis is fully capturing the dynamics that are creating a new Arctic sound propagation environment. Both remain to be monotonically increasing; rays propagated in either of these environments would refract upwards and interact with the surface. While nearly $30 \%$ of ITP data indicates the classical Arctic sound speed, which may potentially align with HYCOM reanalysis efforts in a one-to-one comparsion, $50 \%$ of ITP data indicates a strong ducted environment.
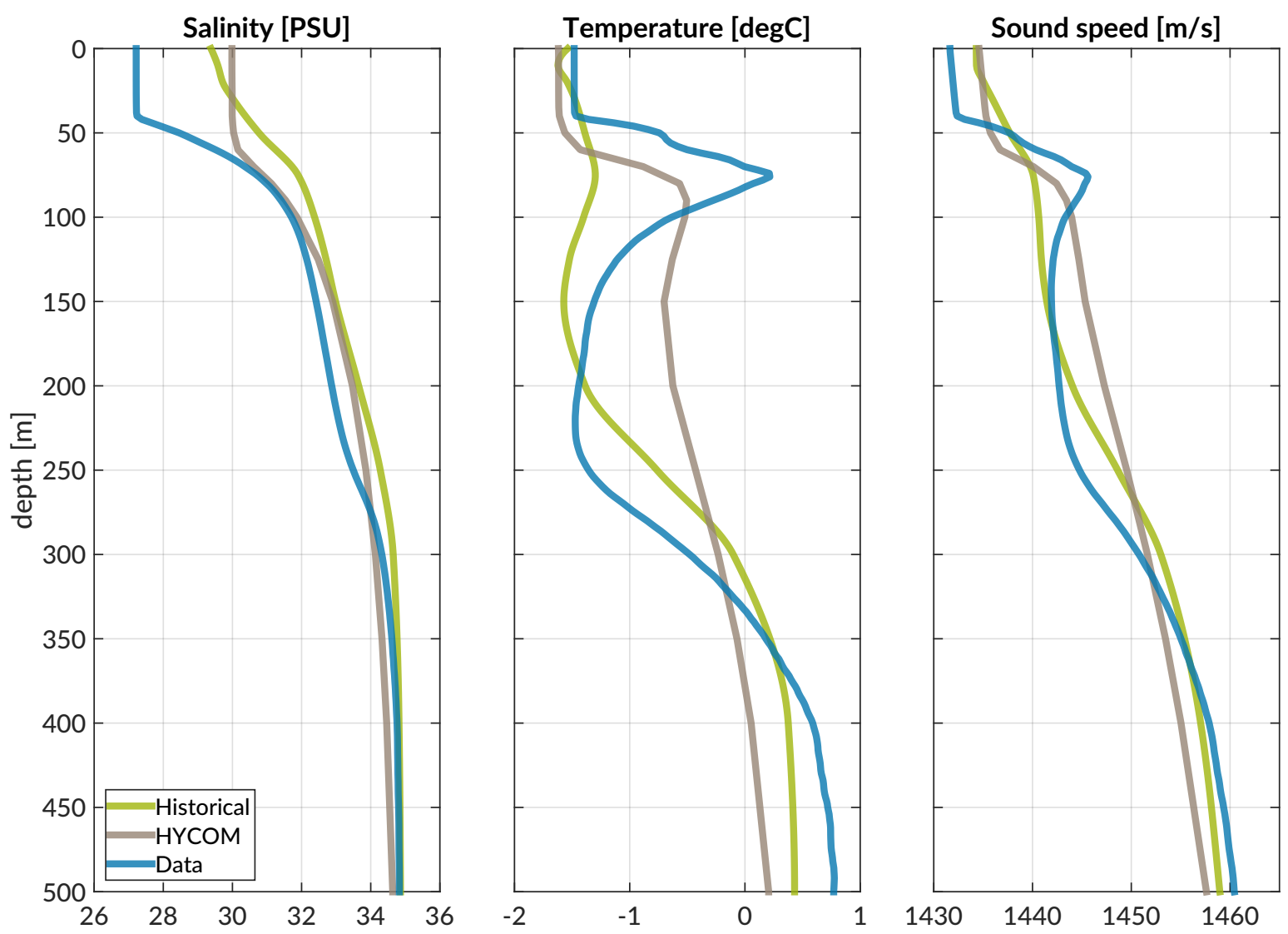

Figure 3.12: A visual comparison of salinity (left), temperature (middle), and sound speed (right) from three input sources: the World Ocean Atlas 09 historical database (green), HYCOM model (gray), and ITP data (blue). The observations shown here are for each input's closest estimate for ICEX16 (March 2016, 73.1N, 149.5W). 


\subsection{Discussion}

The stratification of water masses creating the Beaufort Lens is a recent and profound consequence of a warming Artic. The Beaufort Lens is a widespread, persistent, but not necessarily continuous feature in the Beaufort Gyre, showing interannual variability with minimal seasonal variability. The height of the knee is fairly fixed between $50-65 \mathrm{~m}$, and the ducted conditions strongly correlate with the temperature differential across the duct. The increased warming at the knee height drives a higher local sound speed maximum, which in turn creates a deeper duct. The impact of this temperature gradient on acoustic communications cannot be overstated. While the historical Arctic profile presents a surface refracting and ice interacting acoustic environment, the Beaufort Lens introduces two ducts. The first, at the surface, keeps transmission loss low but severely degenerates signal coherence due to a higher degree of surface interacting paths. The second, approximately between 50 and $200 \mathrm{~m}$ in depth, effectively traps sound above $300 \mathrm{~Hz}$ and can propagate sound at near-basin scales.

Much of the current literature around acoustic studies in the Beaufort Lens dwarfs the scale of AUV operations-on the order of tens of kilometers and over a few days. Compared to historical conditions, transmissions that used to ensonify the operational range and depths now separate into the surface duct or the sub-surface duct, creating a shadow zone. The shadow zone dominates the operational domain, and is a function of the duct strength and source depth. Transmissions between a vehicle and other beacons in an LBL navigation system, already prone to latency and intermittency, might be physically impossible. The wide variance in the duct strength, shown in figure 3.5, further complicates how ducted conditions impact mission-critical communication and navigation. 
The analysis presented here, due to the sparse temporal and spatial density of the ITP platform, is certainly not a perfect snapshot of the Beaufort Lens. But it is a unique and data-forward insight into the duct's overall presence and variability in the region, which previously has been studied with a subset of ITP observations or tomographic experiments. The "big data" approach to statistical learning about the Beaufort Lens is crucial for robotic platform integration, especially because the Beaufort Lens is not well represented in historical data and is not well captured in publicly available high-resolution models or climate models. As the Arctic warms, and becomes more navigable and accessible, there is a direct Navy need to better utilize in situ data, as opposed to HYCOM modeling, for submarine operations.

Given the continuous near real-time data from the ITP system, and the sensitivity of the Arctic to global climate change, these analyses were specifically designed to be automatically updated with the most recent ITP data to monitor changing sound speed conditions. Further monitoring of the Beaufort Lens is an important metric to understanding the changing Arctic for both oceanographic sampling and naval operations.

At the time of graduation, a majority of this chapter forms a manuscript in preparation. Bhatt, E. and Schmidt, H. Characterizing the temporal and spatial variability of the Beaufort Lens from 2006 to 2020. Journal of Acoustical Society of America. 


\section{Tactical Decision Aid framework} FOR ENVIRONMENTAL ADAPTATION

Further exploration of the WHOI ITP dataset showed that the Beaufort Lens is a prominent yet varying acoustic feature that severely impacts the coupled communication and navigation solution. Motivated by a changing acoustic environment in the Arctic Beaufort Sea, this chapter presents a Tactical Decision Aid (TDA) framework for a human decision-maker collaborating with an autonomous underwater vehicle (AUV) to evaluate the vertical sound speed profile. Following the design methodology, ${ }^{1}$ need statements for vehicle deployments in ICEX20 are presented here:

- The vehicle needs an accurate sound speed estimate to seed communication behaviors, which run BELLHOP simulations for a nominal range over all operational depths in the water column to optimize successful message receipt.

- The vehicle needs an accurate sound speed estimate to seed the navigation solution, which currently uses a static value to convert recorded travel time to range.

\footnotetext{
${ }^{1}$ Ulrich et al., Product design and development.
} 
- The topside and vehicle need the same sound speed estimate to corroborate the topside simulation and align the navigation solution.

The need statements align objectives before ideation and specifically do not prescribe any solutions. The implemented design paradigm is presented here in order of operations:

- Empirical orthogonal function analysis will parameterize the sound speed into a handful of weights. This allows for a sound speed estimate to be governed by a small message, which can be sent easily via acoustic communications.

- Various EOF sets will be finalized before the mission duration. This creates redundancy in matching current environmental conditions, as dictated by a CTD, to previously documented ones. The EOF set is chosen once per mission.

- An external CTD informs the sound speed information in the water column. An external CTD mirrors submarine operations, allows for a quality check on the sensor package, and often has a deeper maximum operating depth than the vehicle ${ }^{2}$ to its maximum operating depth.

- A Tactical Decision Aid simplifies the choice of weights for a topside user. Given the nonintuitive nature of acoustic propagation and the statistical abstractions of EOF analysis, a tool to digest the CTD and help a topside user choose weights is paramount to field operation success.

\footnotetext{
${ }^{2}$ Additionally, we are less risk-averse for a CTD to reach its maximum operating depth compared to an AUV
} 
- The integrated communication and navigation solution will include a Group Velocity Computer to run BELLHOP simulations for a nominal depth over a span of ranges to estimate a horizontal group velocity. ${ }^{3}$

The main assumption of this design approach is range independence-that one CTD at topside will be representative of the main acoustic features. This is a valid assumption given an operational scale on the order of tens of hours and tens of kilometers. The temporal resolution is addressed by the ability to send a new update if the local sound speed begins to change. These updates are scalable for range dependence, where updates could correspond to the amplitude and period of EOFs, a geometrical approach of amplitude and extent, or two-dimensional probability distributions. ${ }^{4}$ But providing this extra information may be trivial given the sparse nature of sampling in ice-covered environments. Turning to models to supplement horizontal variability is promising, but publicly available models like $\mathrm{HYCOM}_{,}{ }^{5}$ a sea-ice coupled, three-hour resolution, 1/12 degree resolution model well used by the ocean acoustics and naval intelligence communities currently lacks realism for the Beaufort Lens and its the spatial resolution is $\frac{1}{12}$ of a degree. At these latitudes, that is roughly $4 \mathrm{~km}$, providing only 4-5 grid points for an operational area of 100 square kilometers.

\subsection{Background on Empirical Orthogonal Functions}

Empirical orthogonal functions (EOF) are a common statistical method in ocean acoustics. I use them in this work to parameterize the sound speed input for the TDAs such that a sound speed

\footnotetext{
${ }^{3}$ Randeni et al., "Construction of a high-resolution under-ice AUV navigation framework using a multidisciplinary virtual environment"; Randeni et al., "A high-resolution AUV navigation framework with integrated communication and tracking for under-ice deployments".

${ }^{4}$ Krupskii et al., "Factor Copula Models for Replicated Spatial Data".

${ }^{5}$ Chassignet et al., "The HYCOM (HYbrid Coordinate Ocean Model) data assimilative system".
} 
profile variation is estimated by the weighted sum of a basis function. This section will offer a quick overview on their formulation and existing applications.

\subsubsection{Primer on EOF analysis}

Eigenmethods encompass the Empirical Orthogonal Function, Singular Value Decomposition (SVD), Principal Component Analysis (PCA), among others, with many variants. Eigen is a German word for "own", which also translates to something akin to "characteristic". These analyses seek to determine underlying, characteristic features of a given dataset, often used to study possible spatial patterns of variability and how they might change with time and/or space. As this method is purely data driven, it is not based on any physical principles nor is there any hypothesis based on a probability distribution (however many assume Gaussian behavior for the distribution of principal components).

Given a de-meaned data matrix $X$ of dimensions $N \mathrm{x} P$, where $N$ is the number of observations in time ${ }^{6}$ and $P$ is the number of variables, an EOF analysis calculates the eigen decomposition of $C_{x x}$, the associated auto-covariance of the data matrix, which is by definition, square. This generates $P$ eigenvectors (EOFs or modes) and $P$ eigenvalues, where the sum of the eigenvalues is equal to the total auto-covariance of the data. The modes here are sometimes interpreted as physical structures, but that interpretation should be taken with a grain of salt, because each mode is constrained to be orthogonal to the others.

The ratio between mode $p$ eigenvalue over the sum of eigenvalues indicates "how much variance is explained by mode $p$. Unfortunately, the eigenvalues are not necessarily distinct due to data sampling issues; how many modes one might use to capture the full variability of the initial

\footnotetext{
${ }^{6}$ this can be time, space, or space-time, but I will use time for ease of understanding
} 
data matrix is more of an art than a science. Most analysis of atmospheric and oceanographic processes use the first few modes; many focus just on the first two.

The timeseries of each mode, also referred to as principal components or weights, are found by projecting the derived modes onto $X$, the data anomalies. The PC are organized in an $P \times N$ matrix. An important and useful property is that the PCA timeseries are uncorrelated with each other. Thus, a data matrix $X$ of size $N$ x $P$ can be re-written as a sum of $P$ modes, where further study can isolate any subset of modes. In this abstraction, I will note that the "units" of the variables are preserved in the principal components, not the modes.

It is computationally efficient to perform an EOF analysis through an SVD analysis. The former can compute all the eigenvalues and vectors, which will likely crash your computer for large data sets; the latter computes only the number of eigenvalues and vectors equal to the minimum of $N$ and $P .^{7}$ Given two data matrices, $X$ and $Y$ of the same nature as above, SVD analysis provides a decomposition of both variables to maximize the cross-covariance between them. Thus EOF analysis is the trivial case of SVD analysis when $X=Y$. SVD analysis produces a squared fraction covariance for mode $p$, equivalent to the variance ratio. It also produces singular vectors, equivalent to the principal components.

For the reader, there are numerous resources available to understand and implement EOF analysis. My recommendation would be to stick with one that is relevant to your field of interest, as the terminology across applications is loose enough to be confusing. I would make a personal note, that although generating EOFs is relatively straightforward, it is extremely important to curate the data matrix itself; this is a conversation we often see in machine learning that sometimes slips between the cracks for "statistical" learning.

\footnotetext{
${ }^{7}$ in MATLAB, you can do this as $[\mathrm{U}, \mathrm{S}, \mathrm{V}]=\operatorname{svd}\left(\mathrm{X}\right.$, 'econ' $\left.^{\prime}\right)$
} 


\subsubsection{Summary of EOF ANALYSIS IN OCEAN ACOUSTICS}

In 1980, the seminal paper (it has two references and one is a textbook) on EOF analysis for sound velocity profile (SVP) introduces it as a very compact presentation of the total statistical nature of the SVP data bank. ${ }^{8}$ The authors use 28 standard depths and output a computer model which can generate a new complete SVP for a given place and time.

One of the first applications uses the EOF method to model the vertical structure of sound speed and current fields for the 1981 tomography experiment in the Northern Atlantic. ${ }^{9}$ Since then, some more recent and unique work has merged multiple partial depth data time-series better approximate the mixed layer structure compared to extrapolation, ${ }^{10}$ classified acoustically stable areas, ${ }^{11}$ and reconstructed internal wave trains for acoustic propagation. ${ }^{12}$

While EOFs are incredibly handy for parameterizing SSP perturbations, their coupling to acoustic perturbations is entirely non-linear and unintuitive. Elisseeff et al. introduce a dataassimilation approach to tomography that uses a priori statistics, as opposed to deterministic spatial and temporal structure of the sound speed. ${ }^{13}$ The inverse problem is presented as a fourdimensional field estimation problem, using an oceanographic model, full-field acoustic model, local sound speed measurements, and local acoustic measurements. Xu et al. presents an alternative solution for the environmental-acoustic relationship, looking at system orthogonal functions to optimize acoustic parameterization in adaptive rapid environmental assessment. ${ }^{14}$

\footnotetext{
${ }^{8}$ LeBlanc et al., "An underwater acoustic sound velocity data model".

${ }^{9}$ Cornuelle, "Inverse methods and results from the 1981 ocean acoustic tomography experiment".

${ }^{10}$ Lin et al., "Merging Multiple-Partial-Depth Data Time Series Using Objective Empirical Orthogonal Function Fitting”.

${ }^{11}$ Jensen et al., "Finding Acoustically Stable Areas Through Empirical Orthogonal Function (EOF) Classification".

${ }^{12}$ Casagrande et al., "A Novel Empirical Orthogonal Function (EOF)-Based Methodology to Study the Internal Wave Effects on Acoustic Propagation".

${ }^{13}$ Elisseeff et al., "Ocean Acoustic Tomography as a Data Assimilation Problem".

${ }^{14} \mathrm{Xu}$ et al., "System-Orthogonal Functions for Sound Speed Profile Perturbation".
} 
Notably, Bianco et al. discusses dictionary learning as a new method of generating these sound speed "modes" in a shallow water dataset. ${ }^{15}$ The learned dictionaries have no orthogonal constraint and describe much of the observed variability with one non-zero coefficient. While the framework implemented in these TDAs uses EOFs to compress sound speed information, it is agnostic to the EOFs themselves, and would work on any set of weights and shape functions.

\subsection{Background on Tactical Decision Aids}

On July $3^{\text {rd }} 1988$, in the Persian Gulf, a loss of awareness emboldened aggression and predicated an incredible loss of civilian life. Captain Will Rogers III of USS Vincennes sent a helicopter to aid a Pakistani merchant vessel that had reportedly come under fire from Iranian Revolutionary Guard gun boats. While in radio contact with the gunboats, the helicopter flew over Iranian waters and came under gunfire. Upon hearing this, Vincennes moved to intercept the gunboats and crossed into Iranian waters herself without realizing it. Rogers was granted permission to fire on the Iranian gunboats, sinking two and damaging another.

Amidst the crossfire, Vincennes misidentified Iran Air Flight 655 as an attacking F-14 Tomcat fighter aircraft, even though IR655 was climbing and transponding on Mode III civilian code rather than on Mode II military mode. Rogers issued multiple radio challenges to no response (it is theorized IR655 believed the challenges were for some other plane), and shot down the civilian airliner, over Iranian airspace, killing all 290 passengers and crew on board.

Collyer and Malecki contend that, among the many consequences of this catastrophe was the US Navy's initiation of the Tactical Decision Making Under Stress (TADMUS) program. ${ }^{16}$ The goal of TADMUS was to develop training, simulation, decision support, and display principles

\footnotetext{
${ }^{15}$ Bianco et al., "Dictionary learning of sound speed profiles".

${ }^{16}$ Collyer et al., "Tactical decision making under stress: History and overview".
} 
that would mitigate the impact of stress on decision making. Tactical decision aids themselves were not new to the Navy at this time, as they had been developed in 1972-73 to support mine countermeasures in Haiphong and 25 other North Vietnamese ports and waterways. ${ }^{17}$

Just over a year later, Wagner, a professor of operations research, wrote the authoritative report, Naval Tactical Decision Aids. ${ }^{18}$ A TDA assists a decision-maker by (1) assimilation and convenient presentation on data which of themselves are useful to the decision maker and/or (2) analysis of the tactical problem beyond what is feasible by humans in a timely fashion. Wagner makes an important distinction about TDAs that is prescient considering conversations around ethical artificial intelligence-that no TDA obviates thought, insight, or judgment on the part of the decision-maker, who bears the responsibility for the course of action resulting from the decision.

TDAs, then, provide a scientific basis for executive decisions in a user-friendly and intuitive manner. For naval and/or submarine operations, executive decisions include but are not limited to search methods for anti-submarine warfare, target motion analysis for tracking, information integration for battle group command, and lastly, tactical analysis for environment-dominated systems. This last TDA, where environmental considerations play a dominant role, is the motivation for this work (and also perhaps the least developed TDA mechanism in 1989). Any undersea vehicle operation is significantly affected by the environment, so TDA development for AUVs is a natural analogue for submarine operations.

The Tactical Environmental Support System (TESS) predominantly consists of TDAs whose inputs and outputs are environmental. Wagner highlights four TDAs whose outputs are directly tactical, summarized in table 4.1.

\footnotetext{
${ }^{17}$ Pollitt, "Mine Countermeasures (MCM) Tactical Decision Aids (TDAs), A Historical Review".

${ }^{18}$ Wagner, Naval Tactical Decision Aids.
} 


\begin{tabular}{|c|c|c|}
\hline TDA & Inputs & Outputs \\
\hline \multirow[t]{2}{*}{ NAVSAR } & Navy Search and Rescue & \\
\hline & $\begin{array}{l}\text { target description, last known location, } \\
\text { currents, weather (wind, cloud cover, } \\
\text { visibility), search assets, and search plans }\end{array}$ & $\begin{array}{l}\text { map of search plan locations with es- } \\
\text { timated probabilities that the target is } \\
\text { contained in each grid cell }\end{array}$ \\
\hline \multirow[t]{2}{*}{ ESPA } & Environmental Strike Planning Aid & \\
\hline & $\begin{array}{l}\text { weather conditions such as visibility, } \\
\text { precipitation, rain rate, wind speed, } \\
\text { cloud conditions; sensor inputs such as } \\
\text { sensor type, field of view; target condi- } \\
\text { tions such as type, size, aspect }\end{array}$ & $\begin{array}{l}\text { graphical output of detection, and if ap- } \\
\text { plicable, lock-on regions, by range and } \\
\text { depth }\end{array}$ \\
\hline \multirow[t]{2}{*}{ TESR } & Tactical Environmental Ship Routing System & \\
\hline & $\begin{array}{l}\text { a ship's origin and destination; costs } \\
\text { and user-entered bounds for environ- } \\
\text { mental parameters such as tropical cy- } \\
\text { clone wind, ocean wave height, pitch, } \\
\text { roll, and heave, ocean currents, acoustic } \\
\text { and EM propagation with respect to lat- } \\
\text { itude, longitude, and time }\end{array}$ & $\begin{array}{l}\text { an optimal path, balancing the tactical } \\
\text { cost (a weighted sum across every at- } \\
\text { tribute's cost) against fuel cost }\end{array}$ \\
\hline \multirow[t]{2}{*}{ CHAPPS } & Chaff Planning and Prediction System & \\
\hline & $\begin{array}{l}\text { chaff characteristics, dispenser char- } \\
\text { acteristics, friendly and threat radars, } \\
\text { winds aloft }\end{array}$ & $\begin{array}{l}\text { chaff density and corridor placement } \\
\text { recommendations, and several illustra- } \\
\text { tive graphics, including chaff response, } \\
\text { temporal vertical and horizontal dis- } \\
\text { placement, and chaff dispersion }\end{array}$ \\
\hline
\end{tabular}

Table 4.1: High level summary of environment-dominated TDAs to elucidate the information flow. As a note to the reader, chaff are tiny glass fibers covered in aluminum dropped by airplanes to confuse enemy radar.

TDAs balance the overwhelming nature of improved sensing capabilities with improved processing power. Their development provides a means of experimenting with both the processing and presentation of tactical information to determine what is required to support the submarine chain of command decision making process. ${ }^{19}$

${ }^{19}$ Urban, "Design and evaluation of a tactical decision aid". 
The increasing number of AUVs incorporated into maritime operations was a natural continuation of the TDA framework. ${ }^{20}$ Inputs include the inventory of AUVs, sensors, performance parameters, and operational scenarios. The TDA uses a conservative time-speed-distance and simplified mathematical models to calculate measures of performance for possible assignments of AUVs to missions. The results provide a mission planner with quantitative recommendations for unmanned vehicle mission tasking in challenging scenarios.

One TDA of relevance, due to its acoustic propagation, is the Acoustic Battlefield Aid (ABFA). ${ }^{21}$ It predicts the effects of the atmosphere and local terrain on the performance of acoustical sensors using advanced sound propagation models. Important propagation effects such as refraction by atmospheric wind, temperature gradients, signal coherence reduction, and shadowing by large features are included in the calculations. This TDA hosts interactive and display capabilities to manage acoustical and meteorological properties.

Other TDAs directly tackle the quality of input information. A study from NATO Undersea Research Centre in La Spezia, Italy, uses fuzzy logic to deal with both objective and subjective prior information - the difference between a wave height of $5 \mathrm{~m}$ and a "strong" wave height. ${ }^{22}$ This system assimilates multiple data sources into a data abstraction layer, which then becomes a format independent feature and coverage layer to seed the TDAs. This system was prototyped for deployments off the coast of Florida and the Adriatic Sea to deliver near real-time value-added products for environmental assessment for naval refueling, amphibious landing, diver operations, and heat impact on the ground, and validated in the Adriatic Sea.

While not a naval TDA, a hospital-based TDA has similar implications for mission-critical function. The National Institute of Standards and Technology conducted a demonstration of

\footnotetext{
${ }^{20}$ Duhan, "Tactical Decision Aid for Unmanned Vehicles in Maritime Missions".

${ }^{21}$ Wilson, A Prototype Acoustic Battlefield Decision Aid Incorporating Atmospheric Effects and Arbitrary Sensor Layouts.

${ }^{22}$ Grasso et al., "Geo-spatial Tactical Decision Aid systems".
} 
two prototype TDA interfaces in for emergencies in Wilson Memorial Hospital. ${ }^{23}$ Because many large buildings have automated environmental, security, fire protection, energy management, and elevator systems, a compressed data stream could be vital for transmitting real-time building information to emergency responders. In a simulated fire on the third floor wing of the hospital, participants reacted to information provided by TDAs to safely evacuate residents. The TDAs were well received but participant expressed concerns about standardization, information overload, and reliability.

Indeed, the root idea of TDAs-communicating information between digital and human boundaries-has implanted itself in various other academic fields as computational power and artificial intelligence become more accessible to more hardware platforms. Design space catalogs, generated using parametric models and simulations, have been re-tooled to foster interactive, collaborative, guided, and expert-dependent design processes. ${ }^{24}$

Of course, human-computer interaction drives human-robot interaction, where common tasks are navigation, perception, management, and manipulation. ${ }^{25}$ This is all the more vital as robots have evolved from human-controlled "master-puppet" ${ }^{26}$ servomechanisms to a broad range of robotic mechanisms under minimal human supervision. Because the majority of the next generation(s) of robots will be human-in-the-loop (HITL), either through manual movement or intermittent commands, human factor design is vital to expanding robot capabilities for more sophisticated tasks.

There has been interesting and often times enjoyable work in human-robot trust with respect to autonomous surface vehicles. Establishing human-robot teams for field deployments is

\footnotetext{
${ }^{23}$ Davis et al., Demonstration of real-time tactical decision aid displays.

${ }^{24}$ Brown et al., "Designing With Data: Moving Beyond The Design Space Catalog".

${ }^{25}$ Steinfeld et al., "Common metrics for human-robot interaction".

${ }^{26}$ The original publication uses "master-slave", which is unnecessary, offensive, and technically ambiguous. Wikipedia has an excellent summary on alternatives in practice.
} 
challenging because of marine elements such as wind speed, air temperature, obstacles, ambient noise, and waterproofing. A human-robot speech interface was used to encode commands such that a human participant could escort its robot teammate from a starting to other points of interest. ${ }^{27}$ This same task was performed using the same commands with human teammates as well. While human participants viewed each other in a more favorable light, the robot teammate was evaluated to be "a good teammate" and to "possess adequate decision-making capability" with a p-value below 0.01 .

The human-robot framework was further developed to implement a game of capture the flag, where the tactical decisions and strategies are more complex than simply maneuvering to a point. ${ }^{28}$ Here, the human played against robots of different intelligence levels, where the simple behavior was to circle the flag, and the advanced behavior was to react to an incoming opponent. After the experiment, when asked about which robot they would trust to defend their own flag, in a potential teammate situation, participants overwhelmingly trusted the "advanced" defense behavior over the "simple" one. A follow up study, with more human participants and humanrobot teammates, corroborated that finding-more aggressive (i.e., more human-like) behaviors garnered more trust.

Human-robot interaction has become an important part of marine robotics, especially since deployments are often tethered and/or invisible to human operators. Humans are incredibly good at making complex decisions, whereas machines are much better at complex computation and data storage. Practical applications leverage the best of both through interactivity instead of replacing one for the other. The TDA framework presented in this chapter is operational guidance that changes robot behavior and can be framed as a co-evolutions of the needs from marine

\footnotetext{
${ }^{27}$ Novitzky et al., "A Human-Robot Speech Interface for an Autonomous Marine Teammate".

${ }^{28}$ Novitzky et al., "Preliminary Interactions of Human-Robot Trust, Cognitive Load, and Robot Intelligence Levels in a Competitive Game".
} 
robots with TESS. Thus I style my work after the text- and graphic-based recommendations in CHAPPS with similar physical modeling as ABFA, and implements the ideas of user-entered bounds, assigned costs, and dynamic programming that are integral to tactical decision making.

\subsection{Generalized Virtual Ocean Framework}

This section will introduce a generalized framework for environmentally adaptive behavior in the VO paradigm. We will first give an operational overview and then discuss the cost function that drives the interactive decision algorithm.

\subsubsection{Overview of Operational Procedure}

A schematic detailing the operational procedure into three steps is shown in figure 4.1.

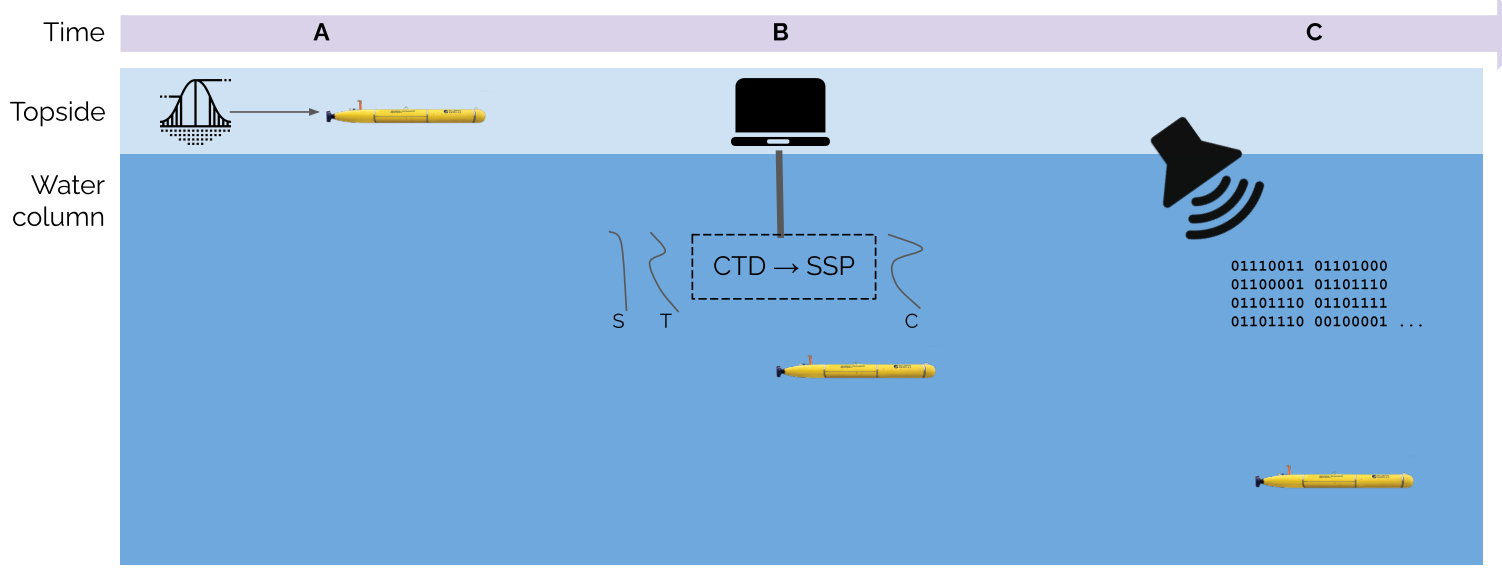

Figure 4.1: A visual diagram of the Virtual Ocean approach. (A) Pre-load the a basis function representations of the sound speed environment for a space-time region of interest. (B) Visualize the environmental realism and acoustic utility of different weight combinations for a human operator. (C) Send the weights via acoustic communication to update the internal sound speed estimate for environmentally adaptive navigation (physical conversion from OWTT to range) and communication behavior (depth adaption). This system enables the same updateable sound speed estimate on topside and the AUV. 
First, in the pre-deployment phase, multiple sound speed training sets are formed. Each training set can be populated by any set of relevant models and/or observations, assimilated to the same depth grid, and de-meaned with a depth dependent sound speed mean. We choose to remove the depth-dependent mean, as opposed to a scalar value, such that the default option for the SSP would still be somewhat realistic. Custom NetCDF VO files are created and and pre-loaded onto the vehicle, where each one contains the depth-dependent sound speed mean; an EOF basis set; a corresponding depth vector; and kernel density estimates (KDE) for the weights for every EOF from the training set.

Next, just prior to vehicle deployment, an initial external CTD (conductivity, temperature, and depth) cast is taken to get a local observation of the SSP in the deployment area. Through the TDA framework, a decision-maker compares this real SSP to the training sets and chooses an initial VO file and weights to best estimate the desired sound speed while preserving historical variability. The basis set is confirmed for the mission duration, and the initial weights are sent to the vehicle via an acoustic message as it begins its mission. This capability is reminiscient of those used for acoustic telemetry for deep ocean moorings. ${ }^{29}$ In the scenario where no EOF set accurately described the SSP, a backdoor was built in to modify the base sound speed value directly. This would impose previously studied variability onto a new mean. The vehicle would begin its mission with all EOF weights set to zero; these weights could be updated accordingly throughout the mission, with reference to the initial anomalous CTD cast.

While the VO file is chosen once per mission, weights can be resent at any time, at the discretion of the topside decision maker. Given another CTD cast, a topside decision-maker can produce new weights and send them to the vehicle, updating the sound speed estimate that drives the environmentally adaptive communication and navigation behaviors.

${ }^{29}$ Catipovic et al., "An acoustic telemetry system for deep ocean mooring data acquisition and control". 


\subsubsection{Interactive Decision Algorithm}

The objective of the interactive decision algorithm is to distill the environmental and acoustic realism for various estimates of an observed sound speed profile. The sound speed estimates are least squares projections of the observed sound speed perturbation with respect to the EOF basis; this implementation iterates through every possible combination of weights to capture the broadest possible estimation space available. Figure 4.2 sketches out the data and simulation pipeline.

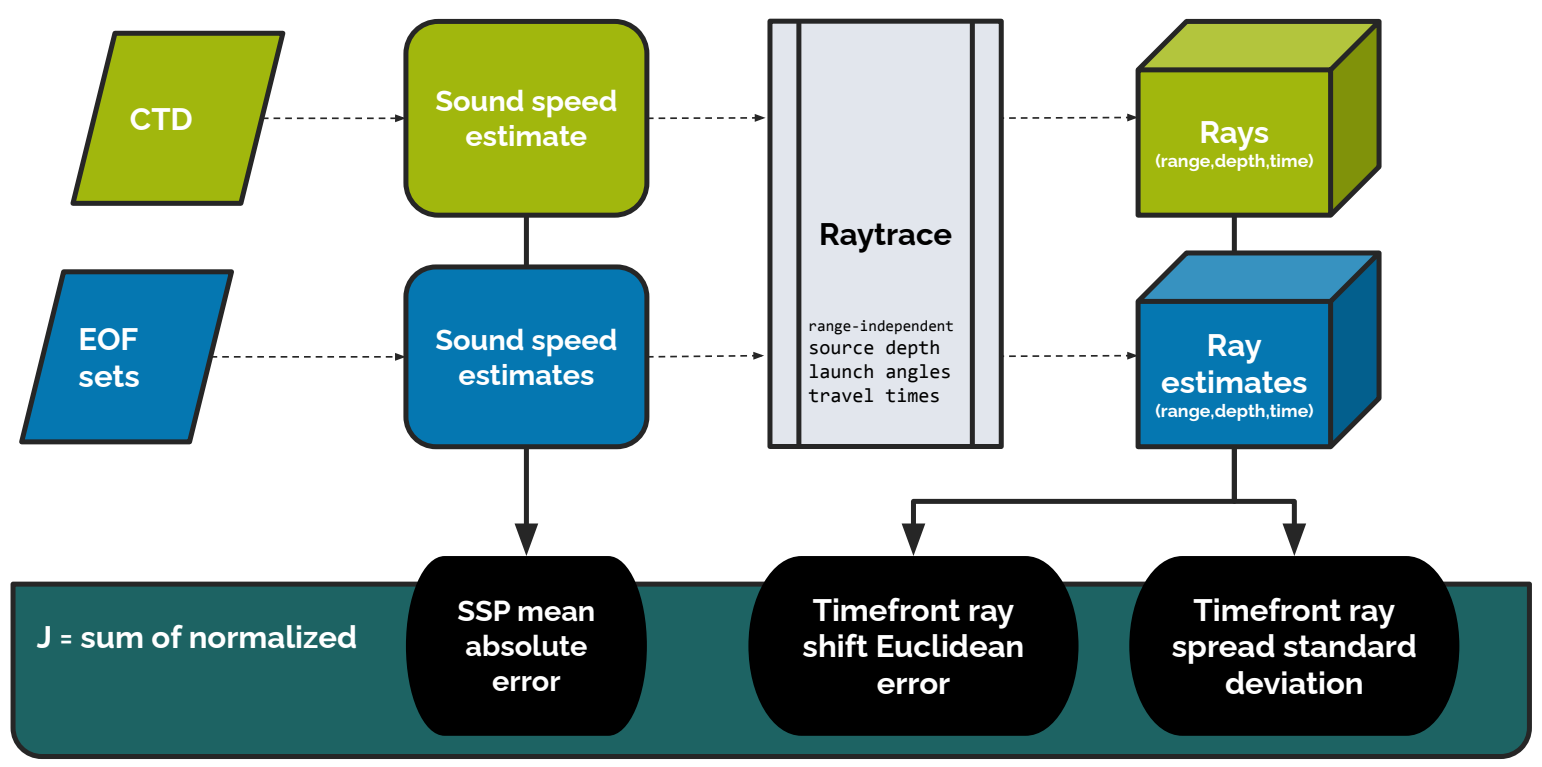

begin interactivity in the TDA

Figure 4.2: A diagram of the TDA to convey joint environmental and acoustic utility given a new sound speed profile via CTD cast. The top layer, in green, shows the flow of real data; the middle layer, in blue, shows the flow of simulated data; the bottom layer, in teal, shows the cost function to compare the two. The parameters for the ray tracing are user configurable.

The environmental realism is assessed by the mean absolute error between the desired SSP (from the CTD) and the estimate (from the EOFs) in the interpolated depth grid from the EOFs 
to the maximum depth of the CTD. The environmental penalty $\epsilon_{c}$ is as follows and preserves the units of sound speed:

$$
\epsilon_{c}=\frac{\sum\left|\hat{c}_{z}-c_{z}\right|}{N_{z}}
$$

Because the downstream application is navigation, the acoustic penalties directly derive from travel times along rays. The ray tracing solution implemented here is a custom function, ${ }^{30}$ which is more computationally efficient than BELLHOP ${ }^{31}$ for this use case, as it does not produce any binary files and only reports the outputs necessary to drive the acoustic metrics. In addition, the simulations themselves take advantage of MATLAB's parfor capability to parallelize function calls to the ray tracer.

Here we define ray shift as the timefront locations of rays of the same launch angle between the desired and estimated sound speeds. The first acoustic penalty, $\epsilon_{r z}$, looks at the magnitude of the error in Euclidean space. The second acoustic penalty, $\epsilon_{\sigma}$, is simply the standard deviation of the ray shift. Both preserve the units of distance in meters. Given a raytracing function, $\Gamma$, that outputs ray positions in range and depth, $P_{r z}$,

$$
\begin{aligned}
P_{r z}(t, \theta) & =\Gamma\left(c, \theta, t_{\text {max }}, z_{\text {max }}\right) \\
\epsilon_{r z} & =\left\|P_{r z}-\hat{P}_{r z}\right\| \\
\epsilon_{\sigma} & =\sqrt{\frac{\sum\left|P_{r z}-\hat{P}_{r z}\right|}{n_{\theta, t}}}
\end{aligned}
$$

\footnotetext{
${ }^{30}$ Jensen et al., Computational Ocean Acoustics.

${ }^{31}$ Porter, The BELLHOP Manual and User's Guide: Preliminary Draft.
} 
Indeed there is no perfect set of algorithms or penalties to unilaterally optimize the choice of EOF weights. The futility of some omniscient artificial intelligence is further compounded by the sensitivity of any operational or tactical scenarios.

Instead, in less than two minutes, the interactive component begins with graphical visualizations (discussed in Section 4.5) and an initial tabular ranking of sound speed estimates. To normalize the differences in scale and units, each penalty is converted to a z-score, with the overall cost function minimizing the sum of all z-scores. A text prompt then encourages the user to investigate any individual ranking further. This ranking is not a greedy estimator-more EOFs do not always perform better. When a new ranking is entered, the cascading series of plots are immediately updated to highlight the chosen rank of interest in teal, compared to the CTD in orange/purple and the other estimates in gray. In practice, this capability was used to "dance" around the rank table, visualizing different combinations and subselecting further ranks of study. The colors and linestyles were chosen to be distinct for individuals with red-green colorblindness or deuteranomaly, visual disabilities that affect roughly five percent of men. ${ }^{32}$

Eventually, a ranking can be selected which preserves the simulation state, visual elements, and initializes (but does not send) the command to update weights on the submerged AUV. An advantage of this algorithm over some machine learning techniques is its emphasis on the observed statistical and simulation state, which places the responsibility for any decision on the authoritative decision-maker and promotes reproducibility and transparency for a chain of command.

\footnotetext{
32Thyng et al., "True colors of oceanography: Guidelines for effective and accurate colormap selection".
} 


\subsection{Execution of Framework for ICEX20}

This section details the execution of the updateable VO framework for ICEX20. This begins with pre-deployment data assimilation to make an EOF basis, covers the CTD casts and the corresponding weights, and ends with a retrospective analysis on the environmental and acoustic penalties. During ICEX20, LT Howard was the primary decision-maker, though the team made decisions by consensus when appropriate.

\subsubsection{Data Assimilation and EOF Analysis}

Six total tuned EOF sets were created using real and modelled data from March of 2013, 2019, and 2020.

Model outputs were provided by the Hybrid Coordinate Ocean Model(HYCOM) Global Ocean Forecast (GOFS) 3.1. ${ }^{33}$ It is important to note that the HYCOM output for 2013 is "reanalysis" data, which assimilates the HYCOM "analysis" with observed temperature and salinity profiles. The HYCOM output for 2019 and 2020 is simply "analysis" data.

Data outputs were provided by the Woods Hole Oceanographic Institution's Ice Tethered Profiler (ITP) program. ${ }^{34}$ Data from all ITP profilers was algorithmically amassed, filtered for spatiotemporal regions of interest, and assimilated to the standard operating depth grid from HYCOM; the automation process ensures that $N \gg P$ for any spatio-temporal subset.

Half of the sets were trained only on modelled data, and the other half blended real data and models. Modelled data is present in every training set to encompass the full water column bathymetry since the publicly available ITP data is limited to approximately $800 \mathrm{~m}$ in depth.

\footnotetext{
${ }^{33}$ Helber et al., Validation Test Report for the Improved Synthetic Ocean Profile (ISOP) System, Part I.

${ }^{34}$ Krishfield et al., "Automated Ice-Tethered Profilers for Seawater Observations under Pack Ice in All Seasons"; Toole et al., "The Ice-Tethered Profiler".
} 
The latter two years, 2019 and 2020, were chosen for temporal proximity; 2013 was chosen for its similarity to trends in Northern Hemisphere sea ice extent provided by the National Snow and Ice Data Center ${ }^{35}$ a few days before ICEX20, as shown in figure 4.3. In the training sets, HYCOM input is limited to the month of March whereas ITP data spills over into February and April, depending on the data density for those years.

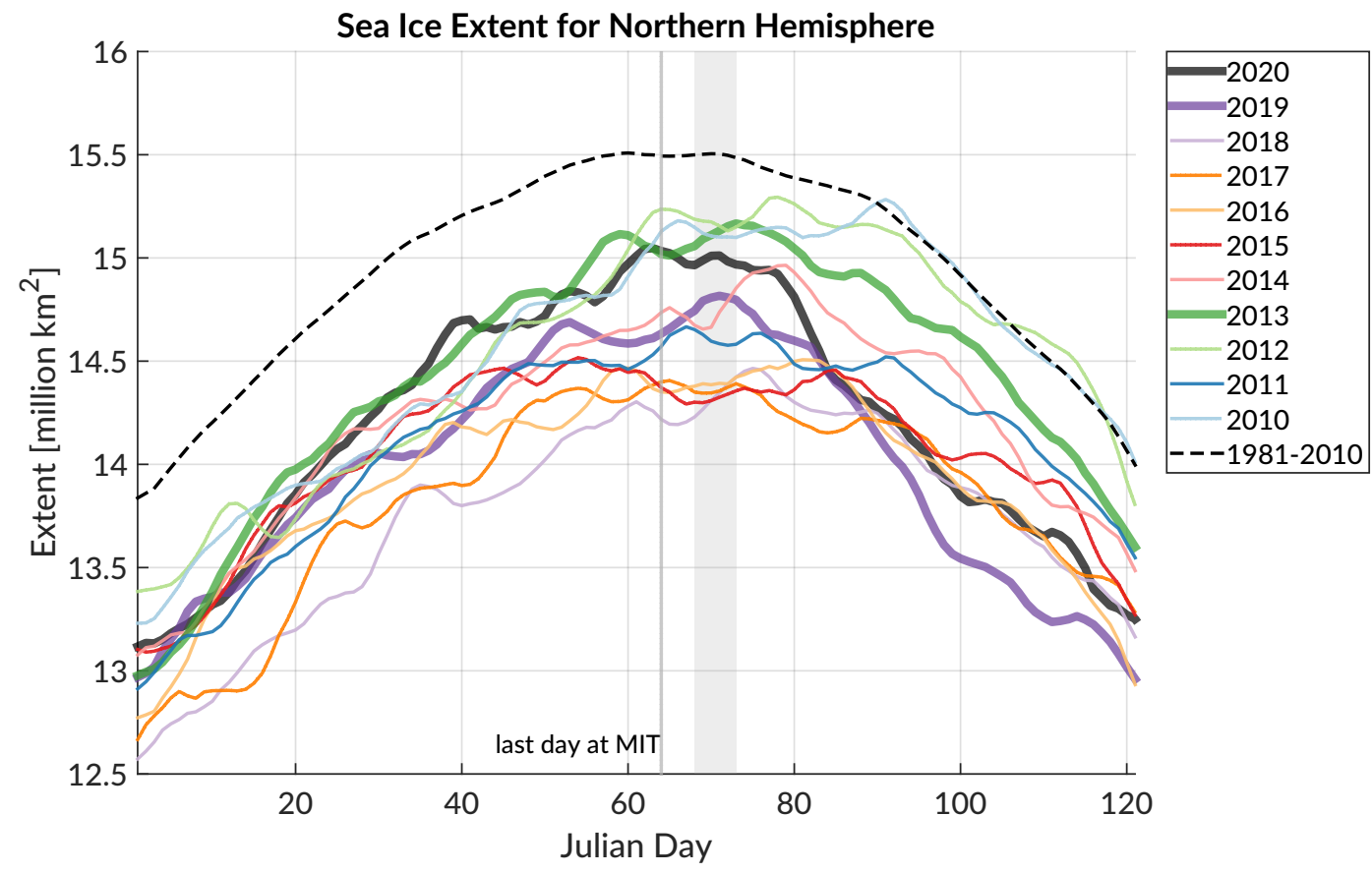

Figure 4.3: Data from the National Snow \& Ice Data Center showing NH sea ice extent for the last ten years. The climatological average from 1981 is in a dotted black line. The years with thicker lines $(2013,2019,2020)$ were chosen, on the last day at MIT, to seed EOF sets for ICEX20.

Figure 4.4 shows the depth dependent sound speed mean for each training set; the ones trained on with ITP data show a Beaufort Lens feature of varying strength, whereas the ones trained without data are reminiscient of the classical Arctic sound speed profile.

For ITP-HYCOM 2013, figure 4.5 shows the first 7 EOF shapes with the cumulative variance explained, and figure 4.6 shows the kernel density estimate from the training set. We smooth

\footnotetext{
${ }^{35}$ Fetterer et al., Sea Ice Index, Version 3.
} 


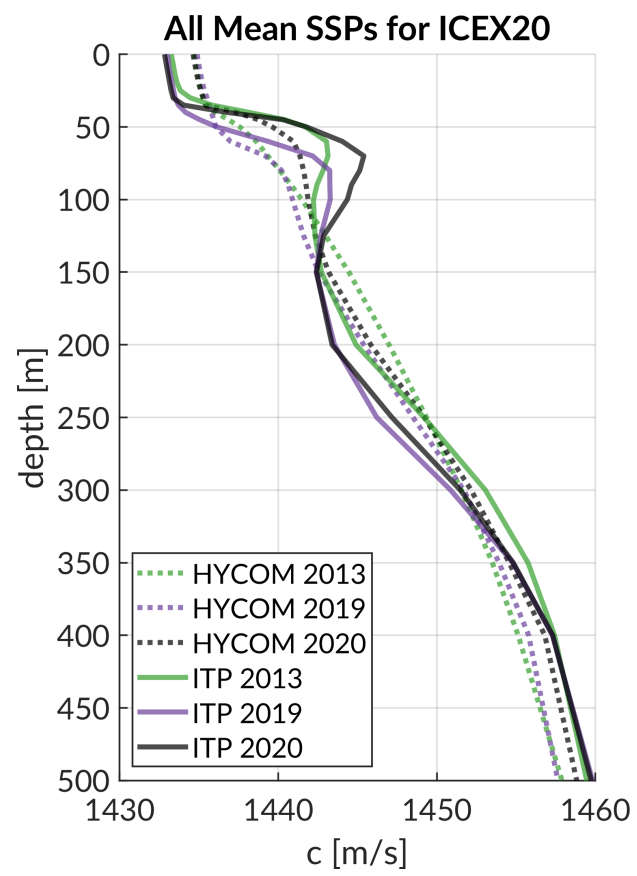

Figure 4.4: The base values for sound speed across the training sets show a variety of duct strengths and heights. The "ITP" label indicates those that were trained on models and data; the "HYCOM" label indicates those that were trained on model output only.

the EOF shapes with a small spatial filter to encourage sound speed continuity at the cost of strict orthogonality. Together, these figures demonstrate that as the variance explained begins to plateau, the associated weight's KDE begins to look more Gaussian and zero-centered. We truncate all of the $\mathrm{VO}$ files to $7 \mathrm{EOFs}$ based on this insight, as higher order EOFs after this may be encoding more noise than signal.

\subsubsection{TDA RAYTRACING PARAMETERS}

Table 4.2 shows the default parameters chosen for ICEX20, where launch angles were informed by prior acoustic simulations to filter bottom bounce paths out and travel times were informed by virtual vehicle runs in LAMSS. These defaults were not modified during the experiment. 

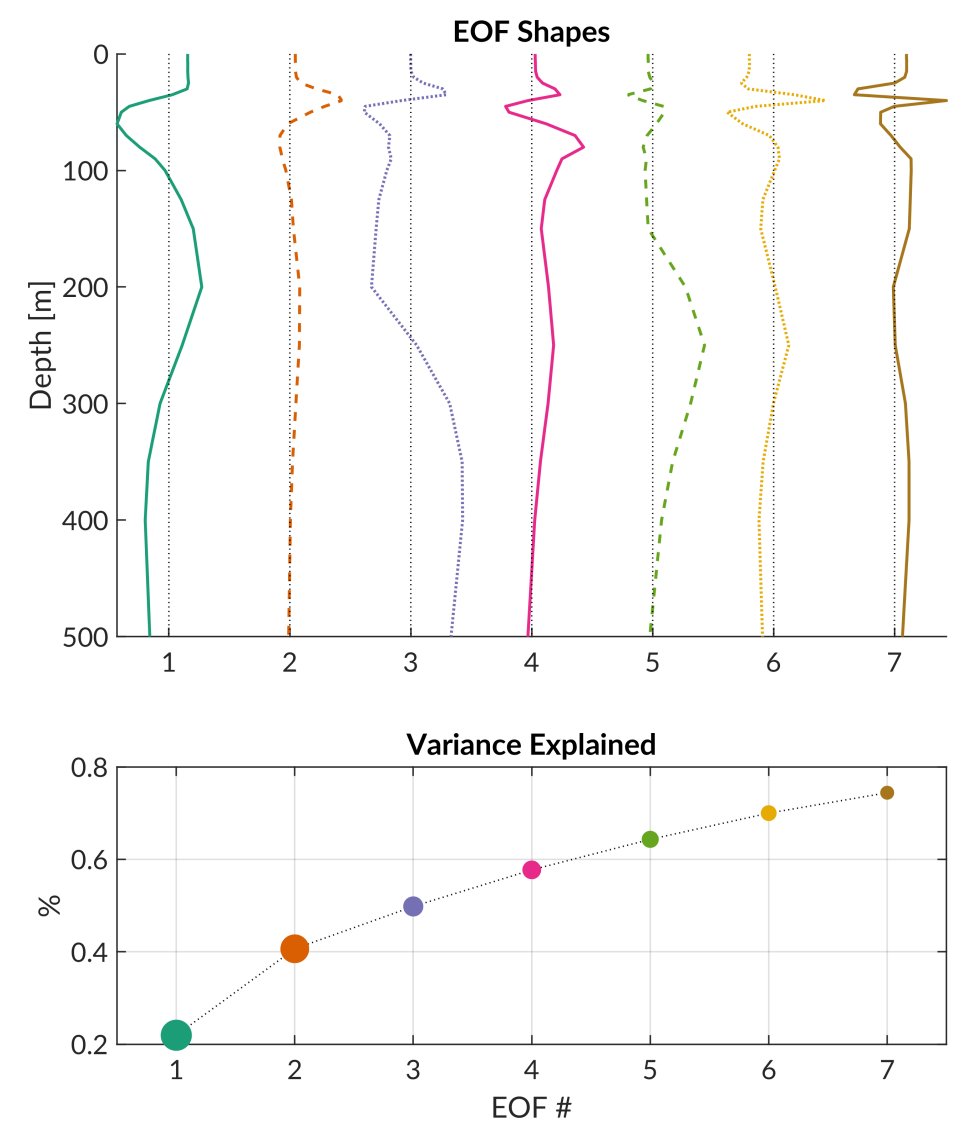

Figure 4.5: The EOF shapes (top) and variance explained (bottom) for the ITP-HYCOM 2013 training set. The EOF shapes themselves actually extend down to the maximum valid depth in the HYCOM $(3000 \mathrm{~m})$, but most of the variance is in the upper $500 \mathrm{~m}$. The shapes shown here are normalized such that the maximum values across all of them are the same.

\begin{aligned} & \hline Parameter Defaults \\ & \hline source depth $[\mathrm{m}] 30 \\ &$ one way travel time $[\mathrm{s}] 0.6,1.3,2,3,4,5 \\ &$ launch angles [degrees] $-30: 5:-15 ;-10: 2: 10 ; 15: 5: 30 \\ &$ bottom depth $[\mathrm{m}] 2680$, sourced from NOAA bathymetry file \\ & step size $[\mathrm{m}] 1 \\ &$\hline\end{aligned}

Table 4.2: Default ray tracing parameters for the TDA. These were selected using simulations of the missions but could be changed by a user in the field. 

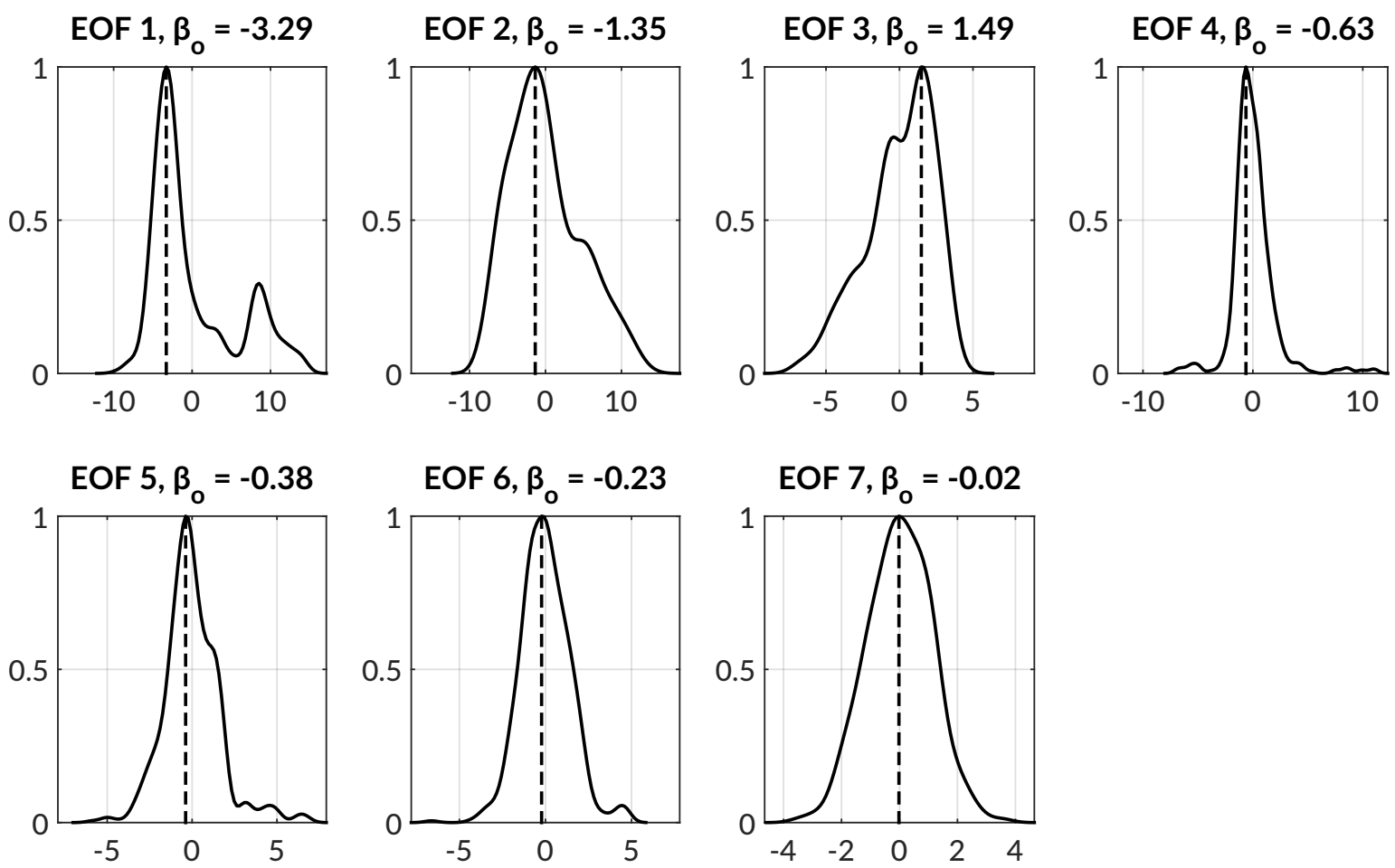

Figure 4.6: The KDEs for each EOF, nominally $\beta$, where $\beta_{o}$ is the maximum likelihood from each distribution.

The ray traces assumes a nominal bottom depth, as opposed to accurate bathymetry, because the coordinate system is variable with respect to bathymetry and the rays of interest are generally not bottom interacting.

\subsubsection{Choosing a Mission EOF Set}

The first choice the TDA provides, based off a driving CTD for a mission (the second CTD in figure 4.8), determines which EOF set will be used to initialize the Virtual Ocean. The tuned EOF sets from ITP-HYCOM 2013 and HYCOM 2019 performed the best, with roughly $20 \%$ and $8 \%$ of sound speed estimates from each having a $\epsilon_{c} \leq 0.5 \mathrm{~m} / \mathrm{s}$. Figure 4.7 shows this analysis-a kernel density estimate for the probability distribution of sound speed error, summed over the water 
column, for all sound speed estimates from the EOF set. The KDE uses a boundary correction such that there will be no non-negative values. This is a flawed and redundant indicator for accuracy, as only one good weight set is needed to estimate the current SSP. The y-axis "nearintercept value" shows the percentage of fits that have close to no error. The visualizations are presented on the order of minutes.

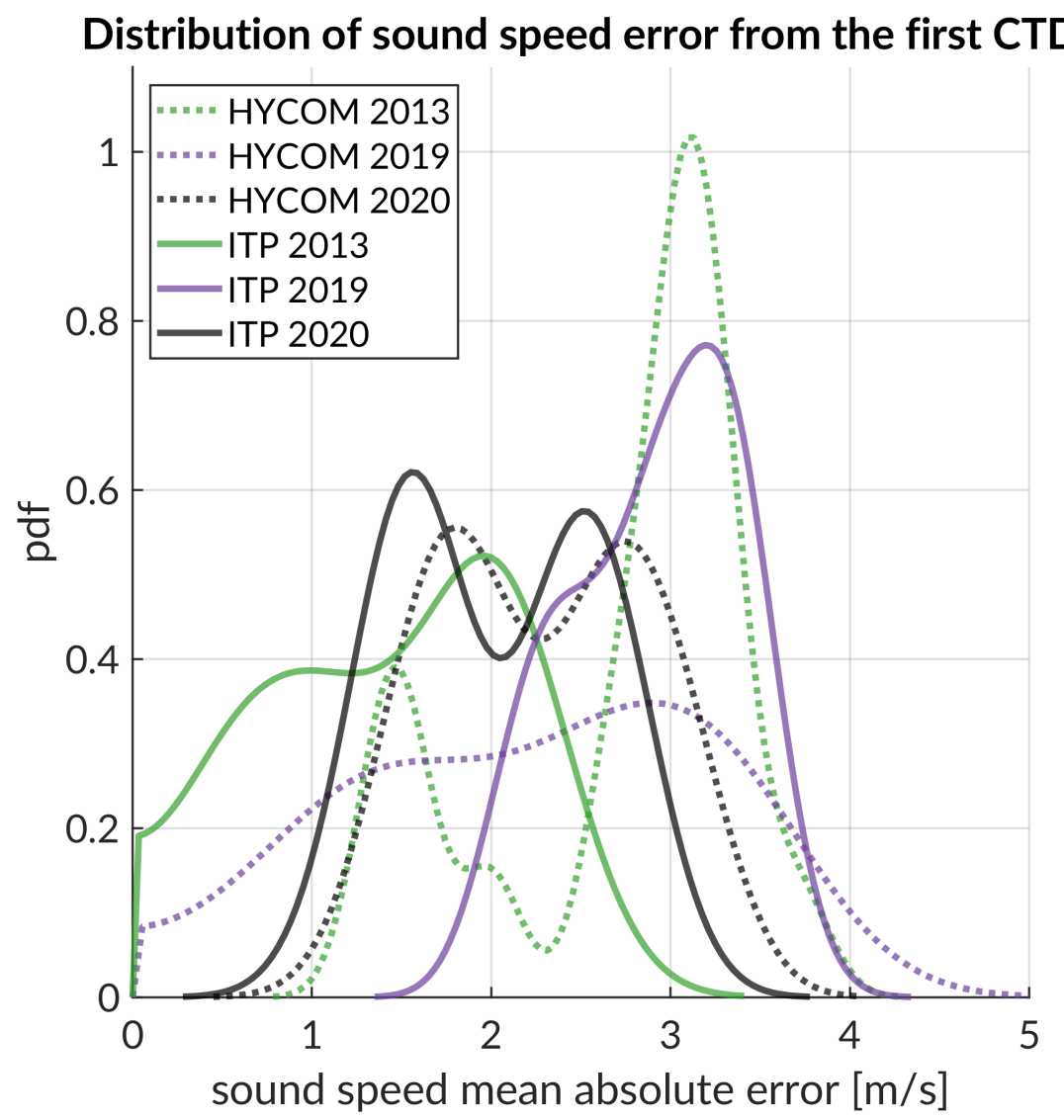

Figure 4.7: To initialize the Virtual Ocean, a user must choose a specific EOF set for a mission based on a CTD. For ICEX20, this was done using the second CTD (the first down to $300 \mathrm{~m}$ ).

The ITP-HYCOM 2013 set was chosen because it had a base value with a prominent duct, whereas that of HYCOM 2019 was monotonically increasing. It is an oversimplification to claim that the sea ice extent strongly correlates with the Beaufort Lens variability, given the differences in spatial scales of the dataset and the sparse sampling nature of the ITP platform. However, 
recent data-forward work by MacKinnon et al. suggests that the growing heat content in the Pacific Summer Water has a first-order effect on accelerating sea ice melt in the region. ${ }^{36}$

\subsubsection{Example of an Initial Tabular Ranking}

The first 20 rankings for a CTD cast on Mar $9^{\text {th }} 0605$, the driving CTD, are reproduced below in Table 4.3. The interactivity developed for this TDA is text-based-it prompts the user with an initial ranking of sound speed estimates and accepts a finite range of ranks to investigate further in a graphical format.

\subsubsection{Acoustic message to update the Weights}

The TDA outputs the command to send a message acoustically using the Dynamic Compact Control Language. ${ }^{37}$ This message is highly compressed, suitable for transmission over a low throughput acoustic channel, by defining bounded field types with customizable ranges and dissolved byte boundaries. A later work ${ }^{38}$ showed greater than $90 \%$ compression compared to a 32-bit integer baseline by using an adaptive probability distribution, but was not implemented for ICEX20 due to both the unproven nature of the EOF approach and the sparse frequency of planned updates. For ICEX20, the bounds for EOF weights were -10 to 10, inclusive, determined by a previous iteration of an EOF training set. For the curious reader, a sample output command to interface with DCCL is shown below:

\footnotetext{
LAMSS_ENVIRONMENT_UPDATE_IN=

${ }^{37}$ Schneider et al., "The Dynamic Compact Control Language", "Unified command and control for heterogeneous marine sensing networks".

${ }^{38}$ Schneider et al., "A State Observation Technique for Highly Compressed Source Coding of Autonomous Underwater Vehicle Position”.
} 


\begin{tabular}{|c|c|c|c|c|c|c|c|c|c|c|c|}
\hline rank & \multicolumn{7}{|c|}{ EOF subset } & $\operatorname{ssp}$ Error $[\mathrm{m} / \mathrm{s}]$ & rayError $[\mathrm{m}]$ & $\operatorname{raySTD}[\mathrm{m}]$ & combo \\
\hline 1 & 1 & 2 & & 4 & & 6 & 7 & 0.418 & 15.51 & 18.89 & 107 \\
\hline 2 & 1 & 2 & & 4 & 5 & 6 & 7 & 0.423 & 15.60 & 18.84 & 124 \\
\hline 3 & 1 & 2 & & 4 & 5 & & 7 & 0.478 & 16.09 & 21.19 & 106 \\
\hline 4 & 1 & 2 & & 4 & & & 7 & 0.471 & 16.13 & 21.47 & 70 \\
\hline 5 & 1 & 2 & 3 & 4 & & & 7 & 0.427 & 17.49 & 24.64 & 101 \\
\hline 6 & 1 & 2 & 3 & 4 & 5 & & 7 & 0.427 & 17.50 & 24.62 & 121 \\
\hline 7 & 1 & 2 & 3 & 4 & & 6 & 7 & 0.427 & 17.49 & 24.65 & 122 \\
\hline 8 & 1 & 2 & 3 & 4 & 5 & 6 & 7 & 0.427 & 17.50 & 24.63 & 127 \\
\hline 9 & 1 & 2 & & & 5 & 6 & 7 & 0.564 & 20.20 & 20.60 & 108 \\
\hline 10 & 1 & 2 & & & & 6 & 7 & 0.559 & 20.69 & 22.09 & 73 \\
\hline 11 & 1 & 2 & & & & & 7 & 0.598 & 20.44 & 21.75 & 33 \\
\hline 12 & 1 & 2 & & & 5 & & 7 & 0.604 & 20.65 & 22.77 & 72 \\
\hline 13 & 1 & 2 & 3 & & 5 & & 7 & 0.559 & 26.34 & 26.04 & 103 \\
\hline 14 & 1 & 2 & 3 & & 5 & 6 & 7 & 0.564 & 26.47 & 26.24 & 123 \\
\hline 15 & 1 & 2 & 3 & & & & 7 & 0.557 & 26.75 & 26.57 & 67 \\
\hline 16 & 1 & 2 & 3 & & & 6 & 7 & 0.562 & 26.85 & 26.76 & 104 \\
\hline 17 & & 2 & & 4 & & & & 1.247 & 15.98 & 11.98 & 15 \\
\hline 18 & & 2 & & 4 & 5 & & & 1.247 & 16.05 & 12.47 & 48 \\
\hline 19 & 1 & 2 & & 4 & 5 & 6 & & 0.624 & 28.69 & 25.75 & 105 \\
\hline 20 & 1 & 2 & & 4 & & 6 & & 0.623 & 28.76 & 25.90 & 69 \\
\hline
\end{tabular}

Table 4.3: The automatically presented ranking based on the environmental and acoustic penalties. From left to right: rank is the $\mathrm{z}$-score sorting of the metrics; EOF subset is which functions are used to generate the estimate; sspError is the mean absolute sound speed error compared to the sound speed derived from the CTD; rayError is the Euclidean distance of specified eigenray timefronts between the CTD sound speed and the EOF estimate; raySTD is the standard deviation of the directional distance of the specified eigenray timefronts; combo is the index for sorting through EOF combinations.

MessageType: "LAMSS_ENVIRONMENT_UPDATE" eof_coef\{ num: 3 coef: $1.41\}$ eof_coef $\{$ num: 5 coef: 9.26$\} \ldots$

\subsubsection{All ICEX20 CTDs}

Ten CTDs were cast from an ice hole over the three and a half day experiment, roughly 250 kilometers northeast of Prudhoe Bay, Alaska. The initial plan was to take a CTD cast every three hours during ICEX20, to mirror the temporal resolution of HYCOM. Due to technical 
difficulties, only ten CTDs were taken, with two different CTD packages. As shown in Fig 4.8, the first five were from the RBR Concerto 3 (RBR), ${ }^{39}$ a retrievable plastic sensor that was operated on a manual winch down to a maximum of $400 \mathrm{~m}$. For temperature, it has an initial accuracy of $\pm 0.002 \mathrm{C}$ and a resolution of $<0.00005 \mathrm{C}$. For conductivity, it has an initial accuracy of $\pm 0.003 \mathrm{mS} / \mathrm{cm}$ and a resolution of $0.001 \mathrm{mS} / \mathrm{cm}$. It has a pressure resolution of $<0.001 \%$ of the full scale.

The latter five were from the Lockheed Martin XCTD-1 (XCTD), ${ }^{40}$ which is expendable but goes to much greater depth. For temperature, it has an initial accuracy of $\pm 0.02 \mathrm{C}$ and a resolution of $0.01 \mathrm{C}$. For conductivity, it has an initial accuracy of $\pm .03 \mathrm{mS} / \mathrm{cm}$ and a resolution of $0.017 \mathrm{mS} / \mathrm{cm}$. It has a depth resolution up to $17 \mathrm{~cm}$.

The accuracy of the XCTD is about an order of magnitude less than the RBR for temperature, conductivity, and pressure, but more than accurate to match the standard depth grid HYCOM provides. The top two meters from all CTD casts were discarded to eliminate observations that drifted warmer due to sensor stagnation in a heated ice hut. For all ICEX20 CTDs, the EOF framework "pins the tail" to supplement the sound speed for the rest of the water column.

Concurrent modeling or data assimilation set a realistic benchmark for the EOF system. The HYCOM reanalysis was interpolated to the exact time, latitude, and longitude of the CTD casts. The reanalysis drastically mischaracterizes the sound speed conditions as the classical Arctic conditions, as there is not a duct nor any significant changes in the SSP throughout the experiment.

\footnotetext{
${ }^{39}$ https://rbr-global.com/products/standard-loggers/rbrduo-ct

${ }^{40}$ https://www.lockheedmartin.com/en-us/products/oceanographic-instrumentation.html
} 


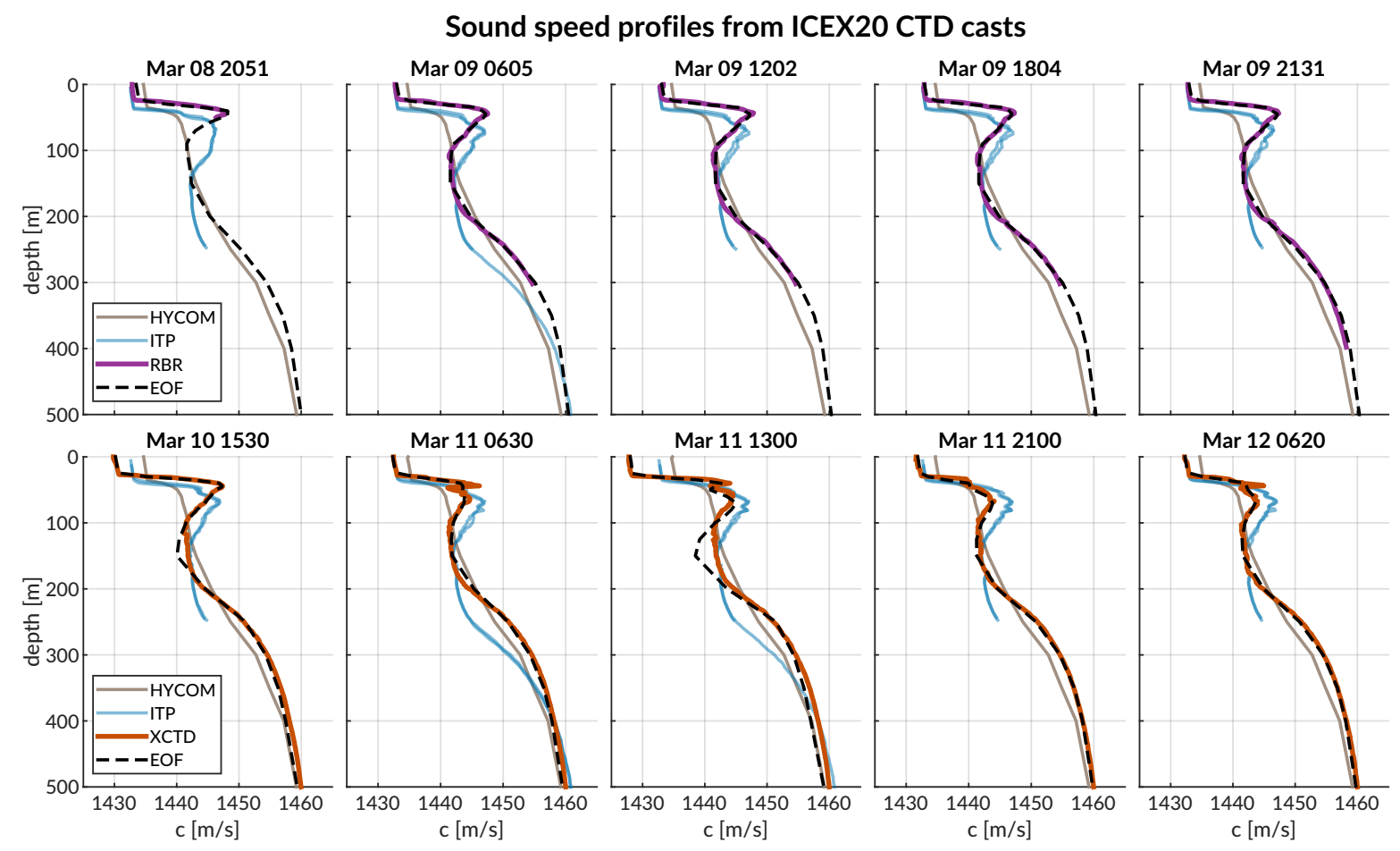

Figure 4.8: All of the ICEX20 CTDs compared to other data streams with the chosen sound speed estimates by LT Bradli Howard. For comparison, HYCOM is interpolated to the exact times, latitude $(71.18 \mathrm{~N})$, and longitude $(142.41 \mathrm{~W})$. The ITP data chooses the nearest 8 profilers in space and time. The initial plan was for a CTD cast every three hours to match HYCOM's temporal resolution; due to technical difficulties, we took 10 over the course of three and a half days, with two different sensors. All times are Alaska time.

The nearest ITP data, weighted by space and time, were retrieved shortly after ICEX $20 .{ }^{41}$ These profiles capture a duct but the lens is $20-30 \mathrm{~m}$ deeper than those of the CTDs. There is better fit in the maximum sound speed of the observed duct, but naturally, due to the sparse nature of ITP observations, they are not sensitive to the local ICEX20 duct weakening, most evidenced from Mar 110630 onward.

\footnotetext{
${ }^{41}$ Without the framework presented in Chapter 3, one would have downloaded data from the two visually nearest ITPs, 103 and 119-103 exhausted its profiling battery but continued to record buoy position, while 119 had a clock firmware issue, thinking it was 1950 and waiting until 20202 to profile (personal communication with John Toole)
} 
The chosen EOF weights, drawn in a dotted black line, more accurately represent the local sound speed conditions and produce reasonable estimates for sound speed beyond the CTD depth as well. The "dominant" SSP for ICEX20, observed in all RBR casts and the first XCTD cast, is extremely well fitted by the EOF set. There is some mismatch around 175 meters, where the depth grid spacing loses resolution-from 100, 125, 150, and then $200 \mathrm{~m}$.

From Mar 11th 0630 onward, the duct develops a micro-lens that continues to shift in later observations. This feature is fairly unstable and not well characterized by the EOF set. Going through each of the four relevant CTDs, the chosen weights ignore the micro-lens; match the micro-lens by misfitting at $150 \mathrm{~m}$; match a weaker duct because of no observed micro-lens; and ignore the micro-lens. Eventually the tactical choice, enabled by the TDA, was to ignore an unstable and acoustically minimal micro-lens. Overall, each sound speed is matched with a mean absolute error no greater than $1 \mathrm{~m} / \mathrm{s}$. The fluctuations across the ten CTDs provide strong narrative evidence that frequent CTD casts are necessary, even for short-term deployments, as the duct changes overnight between Mar $10^{\text {th }}$ and $11^{\text {th }}$.

\subsubsection{Chosen Weights}

Knowing that the sound speed profile was overall well represented, we were interested to see how "predictable" these sound speeds could have been. A comparison of the discrete weights chosen for ICEX20 CTDs, $w$, compared to the distribution of weights from the training set, $\beta$, as shown in fig 4.9, indicates that a bootstrapping technique would have likely captured the SSPs observed.

For all chosen weights, only $\beta_{6}$ and $\beta_{7}$ go beyond the a priori distribution when matching the micro-lens, further suggesting that it is an anomalous feature. The majority of chosen weights fall 
Chosen CTD weights projected onto the tuned distribution

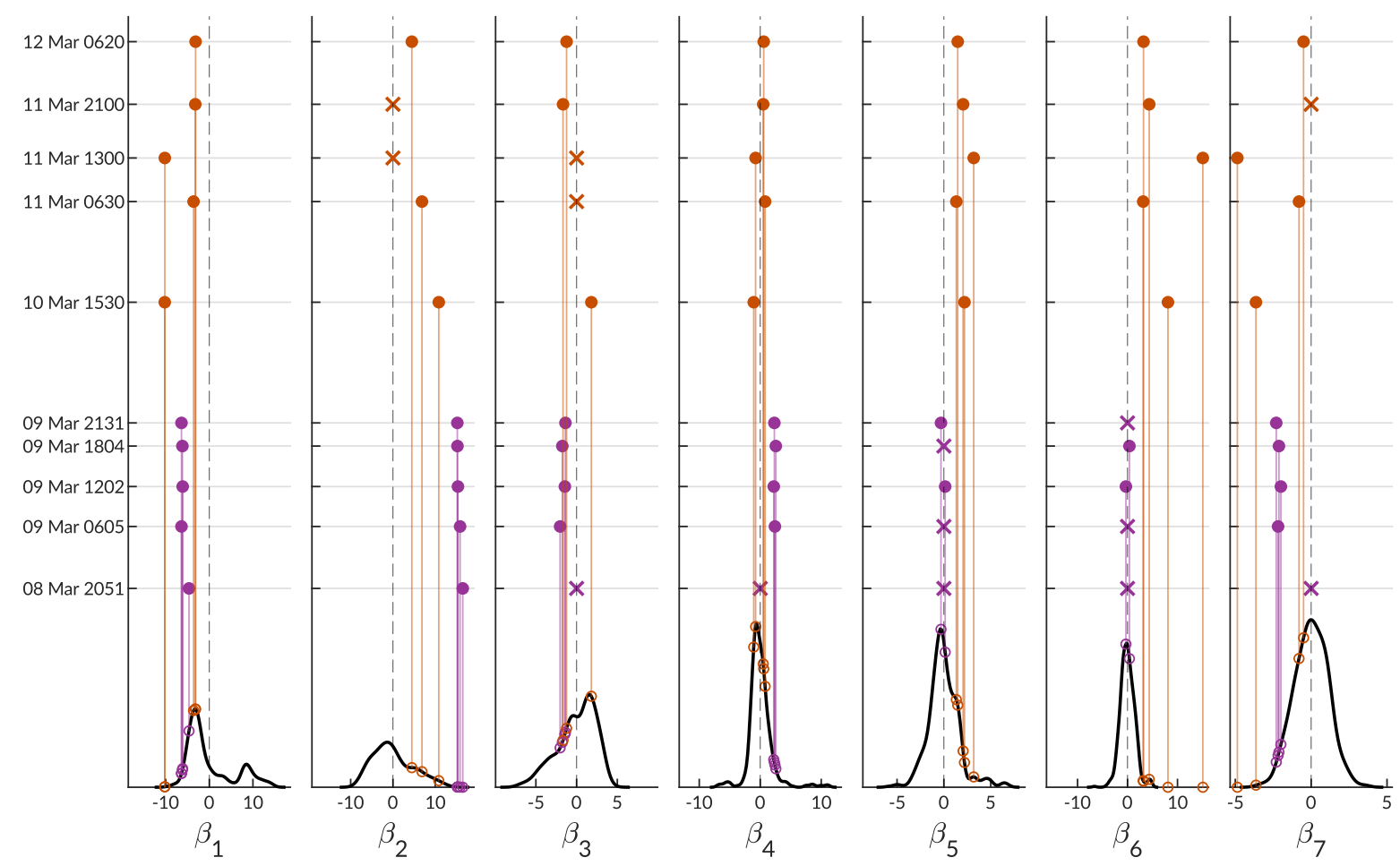

Figure 4.9: A comparison of each CTD's chosen weights, $w$, to the training set distribution, $\beta$. The $y$ axis preserves the timestamp of each weight. The $\mathrm{x}$-axis shows the kernel density estimate for the principal components provided by the EOF training set. Purple dots correspond to RSK and orange dots correspond to XCTD. Weights indicated by $\bullet$ are non-zero (chosen) values, whereas an $\times$ indicates a null value.

not just within the a priori distributions, but within regions of high probability, which indicates that a probability distribution encoding could be practical for future use. ${ }^{42}$

When looking at the temporal spread of chosen weights, we see a consistency in the first five CTDs. The first CTD, which only went down to $50 \mathrm{~m}$, is a narrow exception, as it only uses the first two weights. This was a tactical choice to not overfit the data provided. The sixth CTD, the first of the XCTDs, has slightly different weight mapping even though the SSP is similar; this is because the CTD cast itself is deeper, so the weighting is influenced by a different basis. Future

\footnotetext{
${ }^{42}$ Schneider et al., "A State Observation Technique for Highly Compressed Source Coding of Autonomous Underwater Vehicle Position".
} 
work could add an element of interactivity to the TDA to modify the default uniform weighting of depth grid points. The chosen weights for the rest of the XCTDs are not as consistent, due to ill-fitting of the micro-lens, but show the efficacy of a minimal message to update the internal sound speed estimate on a submerged asset.

As expected, all of the chosen weights use $w_{1}$. But the natural extension of that, that less dominant EOFs might be used less, is not strictly observed as $\beta$ increases. For example, $w_{2}$ is skipped twice; $w_{3}$ is skipped thrice; $w_{4}$ is only skipped once. A simple rank sum for each CTD, across all the estimates that performed better than the mean ranking, finds that the second EOF was the most consistent driver behind the first 8 CTDs; the sixth EOF governed the last two CTDs.

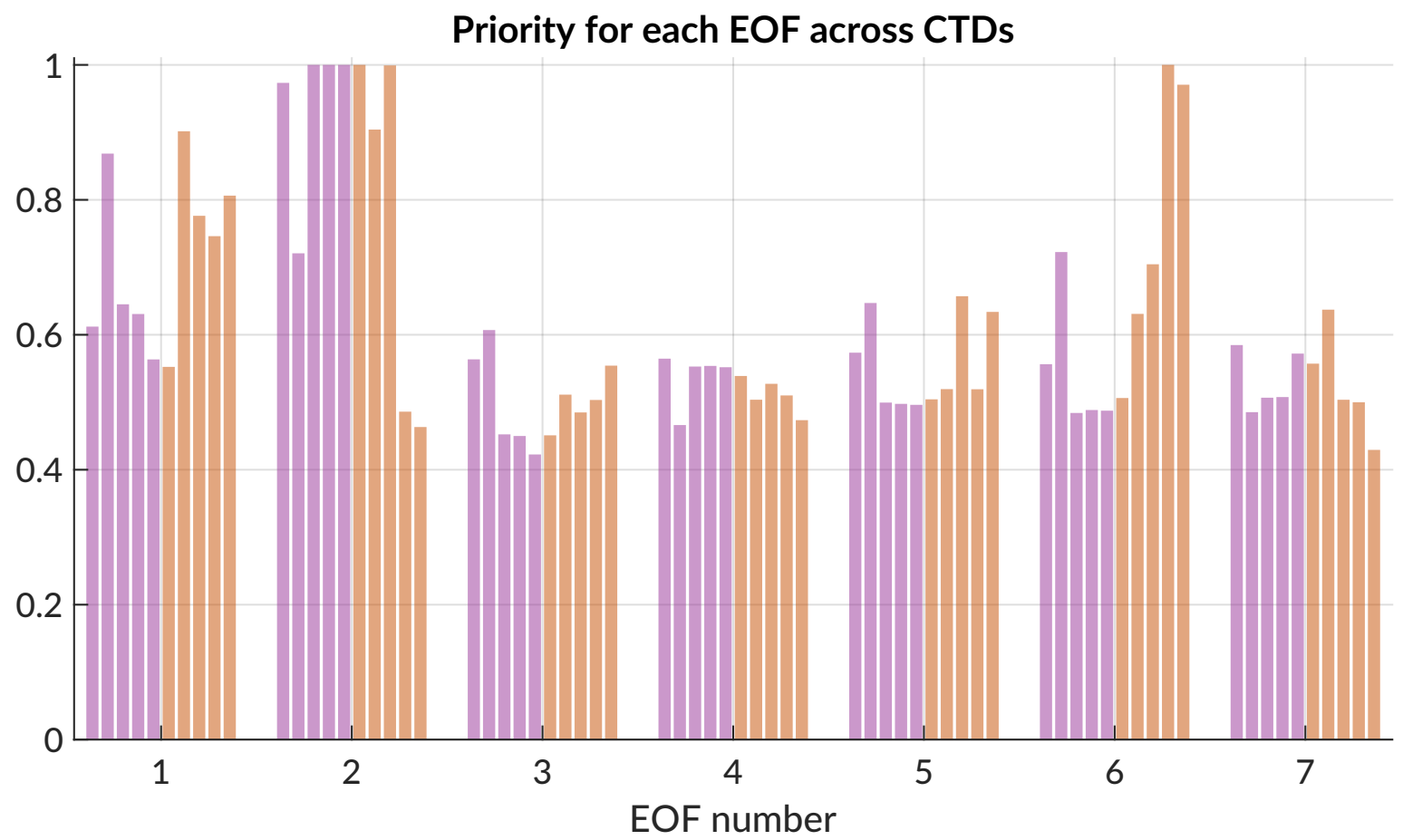

Figure 4.10: Normalized priority for the better-than-mean ranks by EOF number. Each bar (purple and orange) correspond to the 10 CTDs. A value of 1 means that that EOF number was used in all rankings presented. 
The TDA only presents recommendations that perform better than the mean rank (a subset were shown in table 4.3). A topic of conversation during ICEX20 was which EOFs were consistently recommended by the TDA. Those EOFs could be given more credence when deciding on a chosen set. By using a simple rank sum for the proposed ranking for each CTD, and normalizing by the number of ranks shown, a normalized priority by EOF is shown for all CTDS during ICEX20.

EOF 1, for example, while in every chosen weight, was not ubiquitously recommended for any CTD. EOF 2, on the other hand, was the consistent driver behind the first 8 CTDs. The last two CTDs were more governed by EOF 6 . The other EOFs $(3,4,5,7)$ show little variation across the CTDs and amongst themselves. Again, while EOFs themselves are not physical structures, this type of framing can be a useful TDA for elongated deployments, to see consistency across proposed sound speed data structures. It also serves as a warning for always using all EOFs and/or using purely Gaussian distributions on EOFs to synthesize sound speed profiles, as is often done for tomography. Oversimplifying the distribution and variance each EOF explains as a proxy for the significance to a SSP can miss key features. 


\subsubsection{Correlation between Acoustic and Environmental Penalties}

Lastly, a comparison of environmental and acoustic penalties demonstrates the non-linear relationship between the two, and by extension, the necessity for a TDA to convey a joint utility when estimating the sound speed. Figure 4.11 shows $\epsilon_{c}$, the mean absolute error of the sound speed, on the x-axis, and $\epsilon_{r z}$, the ray shift, on the y-axis. The chosen rank is highlighted in the top right and with an $\times$ on the scatter plot.
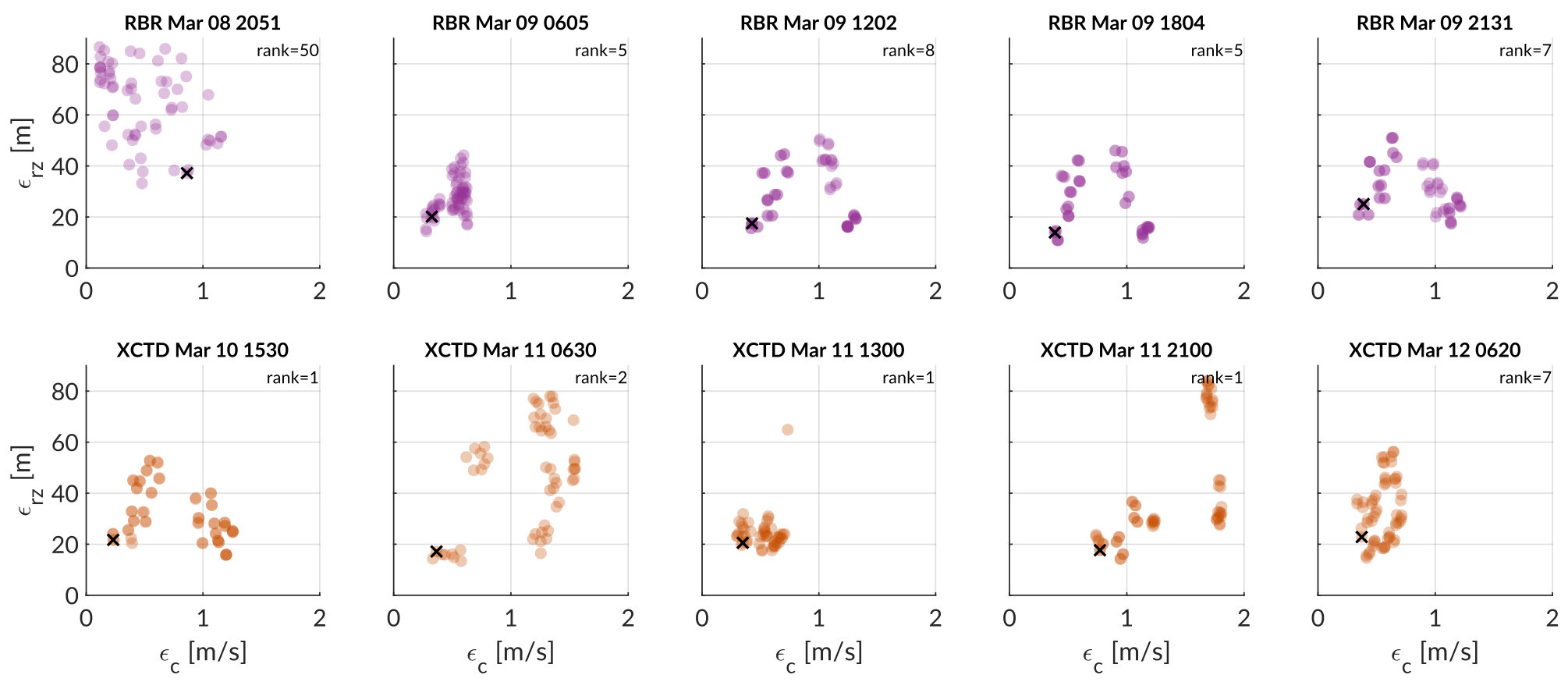

Figure 4.11: The x-axis shows the mean absolute error of the sound speed $\epsilon_{c}$ and the y-axis shows the mean absolute error of the timefront eigenray shift, $\epsilon_{r z}$. The black $\times$ indicates the chosen sound speed estimate's penalties, where its rank is displayed in the top right corner. 
The chosen weights for the first CTD, which has very little depth information, were initially ranked fiftieth. The ranking algorithm does not reward using fewer weights, but presents the information in an easy enough way to find them in the case of a lack of data or mistrust of sensor data. The other ranks chosen have a mode of 1 and a median of 5 , so the ranking algorithm is aligning with decision-maker agreement. Rarely are the chosen ranks the minimal environmental and acoustic penalties. In addition to the mismatch between top ranks, there was a strong instinctual element in the decision maker's process, i.e., simply not liking the shape of a particular sound speed perturbation at a depth of interest, or a preference for acoustic parameters over profile shape in certain operational scenarios. At the same time, the decision maker also settled for some ranks using all EOFs when the difference between top ranks appeared minimal.

There is no singular correlation when examining the relationship between the acoustic and environmental penalty for all CTDs. We can disregard the pattern from the first CTD due to insufficient depth information, though it raises important questions for through-the-sensor mechanisms. The second CTD shows an overall positive correlation, but there are two clusters; the second cluster shows a wide range of acoustic error with little variance in sound speed error. These clusters are the dominant features for the next four CTDs; the first cluster has a positive correlation and the second cluster has a negative one. The negative correlation here most likely stems from turning points in multiple surface-interacting rays re-aligning back to the original CTD pattern. The remaining XCTDs show weak positive correlations between the two penalties, with the exception of the one on Mar 11th 1300, which uniquely has seemingly no correlation.

Overall, this shows the counterintuitive result that a minimal acoustic penalty can be driven by a varying range of environmental penalties. This information was rarely exploited by a human decision-maker for any individual CTD, as they were risk-averse in choosing an environmentally different but acoustically similar environment. The corollary, that minimal environmental 
penalties could produce a wide range of acoustic penalties, is technically true but is generally not possible because of how the EOFs reconstitute sound speed. These penalties are, of course, a function of the source depth and other parameters in the TDA configuration. Continued tactical simulations with the Virtual Ocean for vehicle operations will garner more data about this relationship.

\subsection{Selected Results}

This section will explore three CTD casts to demonstrate the graphical TDAs, with a focus on the environmental and acoustic TDAs, respectively. The first case study uses the first two CTDs (RBR Mar 082051 and RBR Mar 09 0605); the second uses the seventh (XCTD Mar 11 0630). Going through every TDA for every CTD cast would be redundant; the authors are happy to show a live demo of the TDA functionality for any interested reader.

\subsubsection{EnVironmental TDAs}

The first CTD taken on site tested the sensor functionality and the data pipeline; the cast only went down to $50 \mathrm{~m}$. While not used in the field, it, and the CTD cast 10 hours later, provide an excellent window into sound speed estimation beyond sensor depth, and the environmental TDAs.

Figure 4.12 demonstrates the first TDA visualization of the sound speed information. This TDA digests a user-entered sound speed and allows a user to match it to various EOF fits. The test CTD conjures several improbable sound speed estimates because it overfits the minimal data available; the chosen rank is 50 . The driving CTD for the mission, which was 10 hours later, corroborates the sound speed estimate from the previous test CTD. The updated weights 

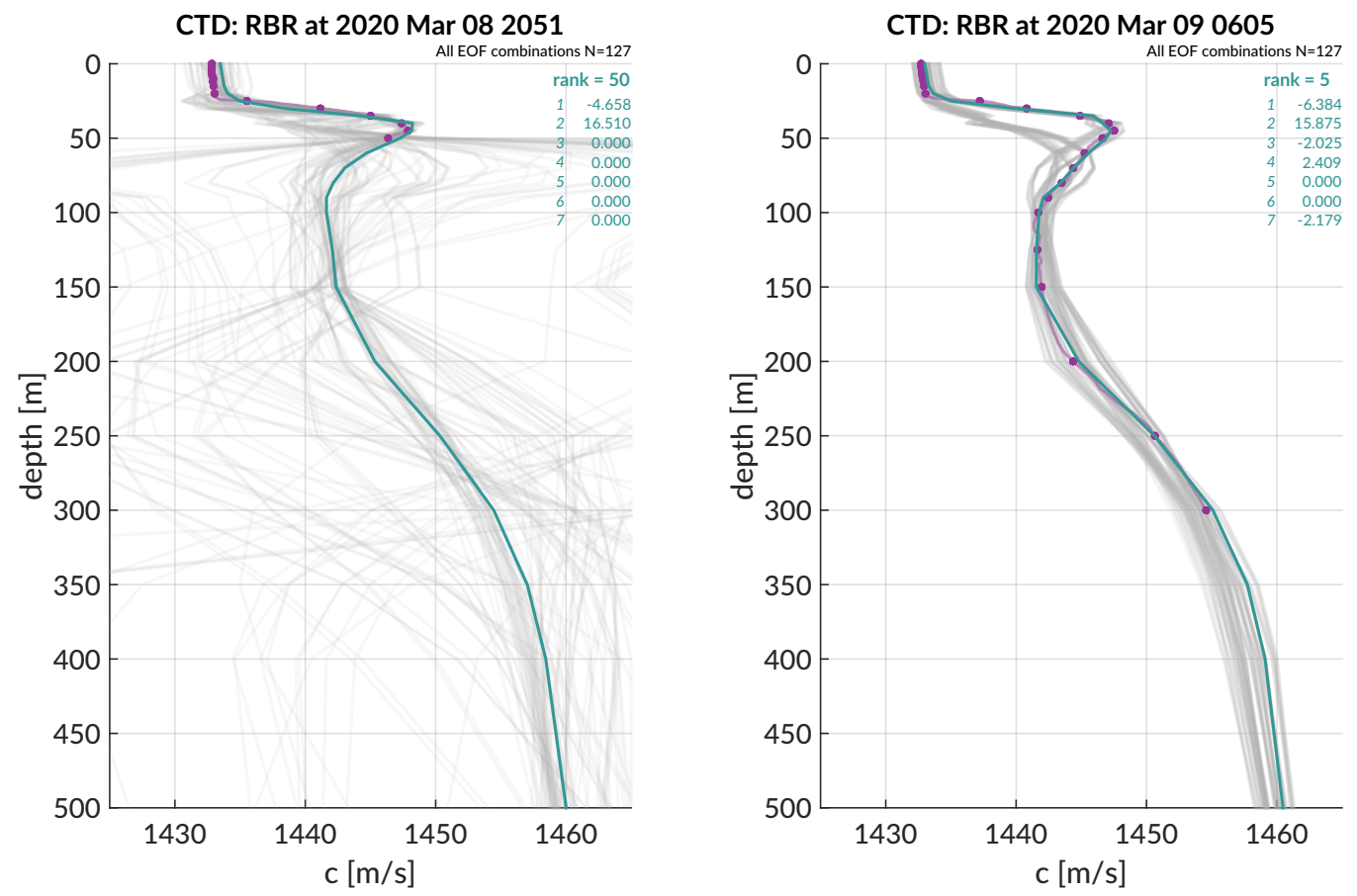

Figure 4.12: A comparison of the first graphical TDA that shows all the combinations of sound speed estimates (gray) compared to the desired sound speed (purple) and the chosen sound speed, rank, and weights (teal). On the left is the pipeline test CTD down to $50 \mathrm{~m}$; on the right is the driving CTD that chose the EOF set. 
modify the first two from before and add the third, fourth, and seventh EOFs to the mix. This better reflects the data, especially between 50 and $100 \mathrm{~m}$; and the TDA decision algorithm does a much better job suggesting this fit, with a rank of 5 . The variation in sound speed fits at the surface encode different warming in the mixed layer; the variation from 50 to $100 \mathrm{~m}$ strangely encodes two different duct shapes (and strengths). At depths greater than $100 \mathrm{~m}$, the window of the sound speed estimates at any given depth is roughly $5 \mathrm{~m} / \mathrm{s}$.
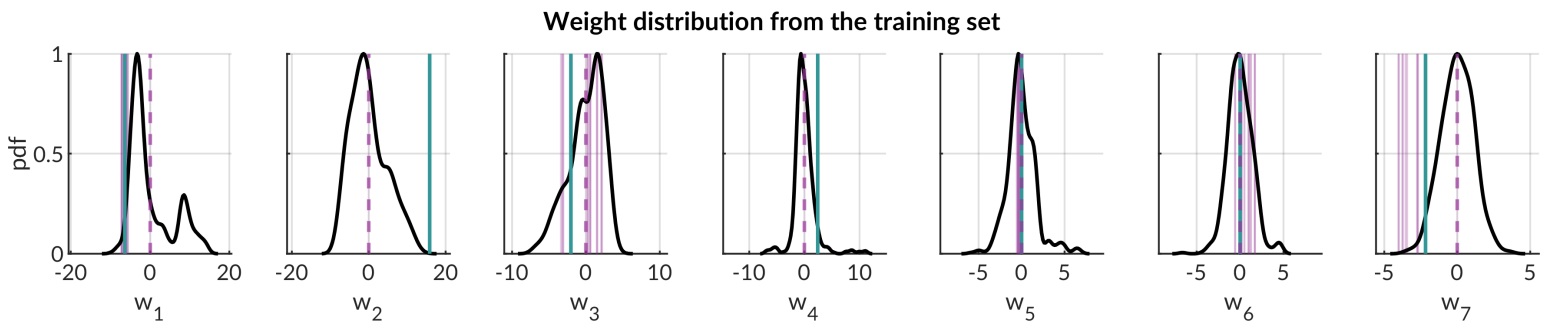

Figure 4.13: This TDA shows a priori weight distribution from the EOF training set in black. Purple lines show the weights driving all sound speed estimates and a teal line shows the weight for the chosen rank. The purple dashed line refers to weights that were manually tapped out as null.

The second environmental TDA, shown in figure 4.13, presents the discrete weights for all sound speed estimates, $w$, versus the weight distribution from the training set, $\beta$. LT Howard, and many in the ICEX20 team, were interested in sending the weights that most heavily and consistently deviated from the maximum likelihood. This is seen quite clearly for $w_{1}, w_{2}$, and $w_{7}$, where the weights have little standard deviation and are located in a tail of the distribution. Less importance was attributed to a weight like $w_{3}$, where the estimates spanned the full distribution (suggesting little impact), or $w_{5}$, heavily centered at 0 (suggesting unnecessary forcing).

Overall, these two TDAs characterized the local Beaufort Lens and the error in the sound speed estimation while contextualizing the estimate relative to the training distribution. 


\subsubsection{Acoustic TDAs}

The second case study examines a weaker duct with the micro-lens around $45 \mathrm{~m}$. This particular CTD is a compelling case study for the acoustic TDAs because the chosen weights do not try to fit the micro-lens.

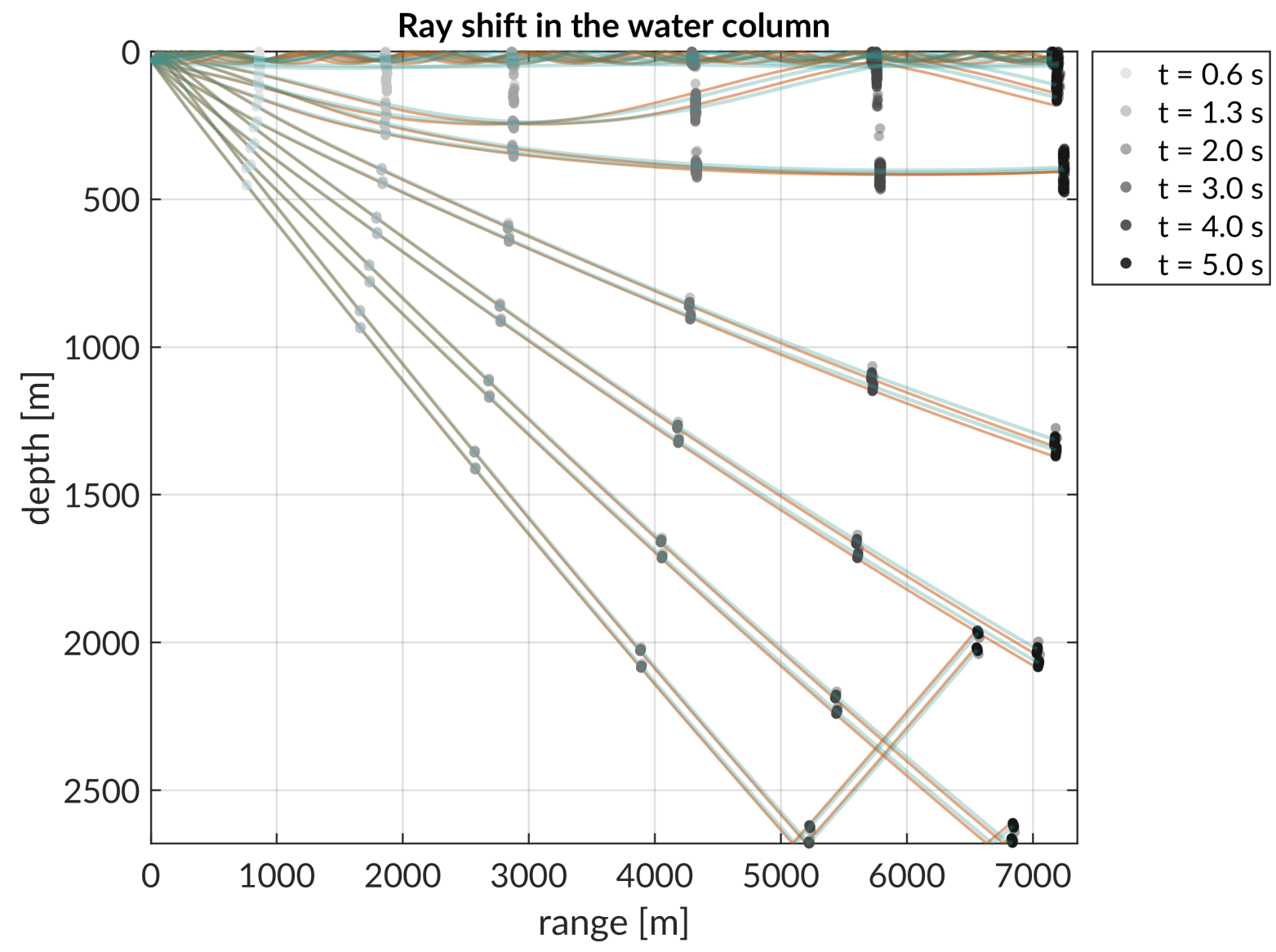

Figure 4.14: This TDA displays the raytrace for the CTD in orange and the EOF sound speed estimate in teal. The legend indicates timefronts for all sound speed estimates, with color darkening with increasing travel time.

The first acoustic TDA is a visualization of the raytrace that drives the acoustic penalties. The user-entered time fronts are highlighted with increasing color to visualize the spread, but will be further explored in the second acoustic TDA. The ray comparison by color and transparency 
is more intuitive than comparing color as an indicator for transmission loss across two environments. The difference in transmission loss is effective, but is a confusing proxy for navigation. In figure 4.14 , one can see very little ray shift throughout the water column, even though the micro-lens is ignored. The multi-path is well preserved; the rays that bound the shadow zone are slightly extended but not in any way to mislead position. The deeper rays are also preserved, but were generally not considered to be of high importance because of the operational ranges of $4000 \mathrm{~m}$ and depths of $300 \mathrm{~m}$. A decision-maker only trying to fit the sound speed environment, as EOF analysis often does, may have overweighted the impact of the micro-lens because it is visually prominent.

The second acoustic TDA simplifies the ray shift over all launch angles by the suggested timefronts, as shown in figure 4.15. Here we define a unitless ray shift, where range is normalized by the distance the ray has traveled, and depth is normalized by the maximum water depth. The orange dot, referencing the desired ray position, centers the ray shift with no error. The numerous teal dots indicate the ray shift for all prescribed launch angles. The transparency of the gray dots, referencing the ray shifts for all other sound speed estimates, is weighted by the proximity to other ray shift estimates. This TDA allows a decision maker to discriminate based on perceived OWTT, i.e., tightening the arrival structure of 1.3 seconds might be tactically more important than the arrival structure at 4.0 seconds. As a byproduct, the TDA gives a nominal estimate for the navigation error as well that is strictly derived from the range propagation, as opposed to other sources of error in positioning algorithms. 
Relative ray shift
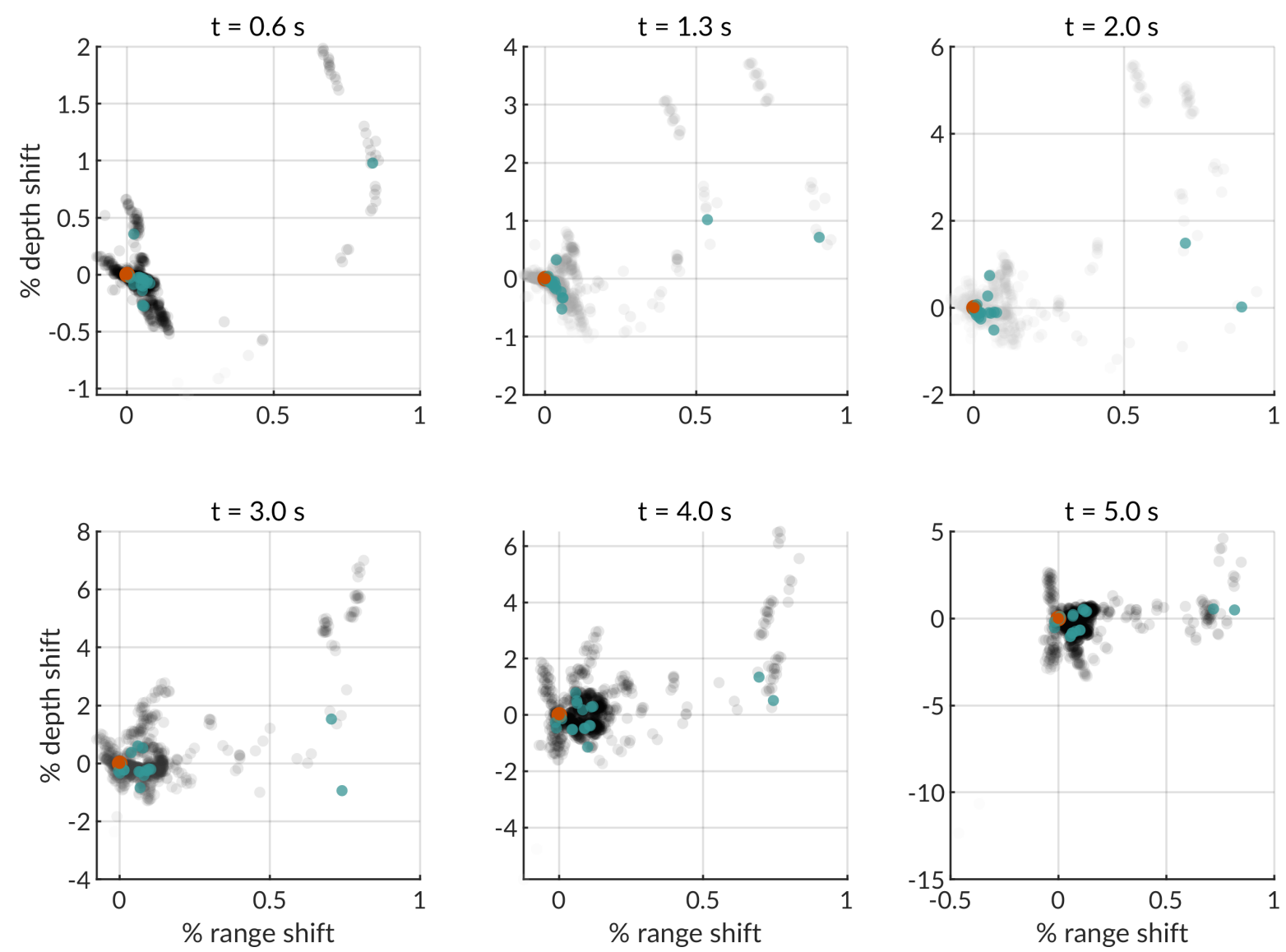

Figure 4.15: This TDA displays the relative timefront eigenray shift as a percentage. The $\mathrm{x}$-axis is the range error relative to the range traveled and the $y$-axis is the depth error relative to the water column depth. The gray dots show the relative ray shift for all sound speed estimates and are darker for regions that have higher probability. The teal dots show the relative ray shift for the chosen sound speed estimate for each launch angle. The orange dot is centered at $\langle 0,0\rangle$, as this is the reference from the CTD.

\subsection{Discussion}

Based on our Beaufort Sea demonstration during ICEX20, we conclude that this framework sufficiently addresses the need to consider the acoustic impact of a real time, dynamic SSP in current underwater navigation paradigms. We have demonstrated that this tactical decision aid succinctly and effectively enables the use of an updatable, in-situ, full water column profile rather 
than a single deterministic value for sound speed. The TDA design is derivative of existing naval operational paradigms and meshes with related academic fields where interactive "dialogue" between humans and computers enhance or optimize outcome. The environmental TDAs assimilate and conveniently present data, which of themselves are useful to the decision maker. The acoustic TDAs provide an analysis of a tactical problem beyond what is feasible by humans in a couple minutes. The complexity between environmental errors and acoustic errors creates a decision space that is otherwise wrought with conjecture. By embedding acoustic error into the ranking, the search domain is realigned and simplified for operational use. In doing so, our approach enables a human decision-maker to collaborate with an AUV in a way that promotes environmental battlespace awareness and trust in autonomous capabilities.

While EOF analysis is widely adopted for sound speed reconstruction for its simplicity and effectiveness in capturing variability, it would behoove researchers to be cognizant of acoustic implications. In this work, we smooth EOFs to encourage sound speed continuity and implement an acoustic fit in addition to the usual environmental fit to assess weights. The lesson learned on this subject is that while a certain set of weights may produce a different overall shape from the latest sound speed profile, it may actually be a closer match in terms of acoustic properties. In order to make this distinction clear to the human decision-maker, some pre-training on the impact of the acoustic parameters will be required prior to use. Because of mismatching depth grids of ITP data, the standard HYCOM grid was implemented for the EOF sets, to simplify any performance comparisons between them. A finer depth grid is likely to do better, up to some threshold at which the variability it encodes begins to match more noise than signal. Lastly, six EOF sets was an arbitrary number to build redundancy that balanced the constraints of the timeintensive task of first deciding on an EOF set for the whole mission versus cataloging ducted 
and non-ducted environments. Future deployments should focus on the blended approach and include as many years as are relevant and/or possible.

TDAs, and the artificial intelligence they employ to inform any human user, are becoming a staple in mission critical tasks. The processing that goes on behind the scenes is an important capability enhancer for military and scientific applications. In an increasingly complex tactical environment, artificial intelligence will best assist naval decision-making in a system of systems approach, where each initiative is iterated on independently but fits within a modular operating paradigm. ${ }^{43}$ This is an exciting field of study, connecting the dots between:

- "known knowns"-statistics, reporting, news

- "known unknowns"-data mining, forecasting, search engines

- "unknown knowns"-knowledge discovery, collective memory

- "unknown unknowns"-machine learning, game theory, big data

Given the Arctic's heightened sensitivity towards global climate change, we expect the spatiotemporal variability of the Beaufort Lens to continue to be a focus for oceanographers, acousticians, and roboticists alike. In future work, the approach presented here, which adapts to temporal changes in the sound speed, should be expanded to account for spatial variability - especially for long range applications. One promising avenue for spatial mapping may be two dimensional probability distributions. ${ }^{44}$ Additionally, machine learning techniques like dictionary learning ${ }^{45}$ may simplify weight finding and even reduce message size. In light of the SSP estimation mismatch at 150 to $200 \mathrm{~m}$ in depth, there is a need to understand what depth grid resolution is

\footnotetext{
${ }^{43}$ Johnson et al., "Artificial Intelligence - An Enabler of Naval Tactical Decision Superiority".

${ }^{44}$ Krupskii et al., "Factor Copula Models for Replicated Spatial Data".

${ }^{45}$ Bianco et al., "Dictionary learning of sound speed profiles".
} 
optimal to encode the variability seen in the Beaufort Lens. It is also of extreme importance to maintain the quality of data garnered in the Canada Basin to continue to study the potential interactions between sea ice and the Beaufort Lens. ${ }^{46}$ Because satellite data can be at daily resolution, linking the two may be a way to leverage the information disparity to further study warming of sub-surface Arctic waters. More broadly speaking, the sea ice thickness dictates when these experiments can be done safely (generally only in March); but its changing nature threatens these types of efforts and even invites a different ambient acoustic environment. ${ }^{47}$

As we seek to transition from teams of humans supervising a single vehicle towards teams of vehicles collaborating with little human supervision, a key focus of this work is building and maintaining operator trust in embedded AUV intelligence. Operations in the Beaufort Lens provided an excellent testbed to develop and demonstrate a new type of information sharing that significantly improved autonomous navigation and communication behaviors. We hope this work enables more intelligent multi-vehicle sampling, with geolocating oceanographic observations, to better understand the evolution and dynamics of oceanographically sensitive and acoustically complex environments.

The TDAs presented in this chapter address a critical gap to consider an internal sound speed environment on a submerged asset for environmentally adaptive communication and navigation. The next chapter will focus on the performance enabled by this capability.

At the time of graduation, a majority of this chapter forms a manuscript in review. Bhatt, E., Howard, B., Schmidt, H. A Tactical Decision Aid Framework for Environmentally Adaptive Navigation and Communication in Autonomous Underwater Vehicles. IEEE Journal of Ocean Engineering.

\footnotetext{
${ }^{46}$ MacKinnon et al., "A warm jet in a cold ocean".

${ }^{47}$ Chen et al., "Spectral, spatial, and temporal characteristics of underwater ambient noise in the Beaufort Sea in 1994 and 2016”, “Temporal and spatial characteristics of the Beaufort Sea ambient noise environment”.
} 


\section{ReAL-TME ACOUSTIC RAM RNGE}

\section{ESTIMATION DURING ICEX20}

This work presents the results from, to my knowledge, the first field deployment of in situ acoustic ray tracing for range estimation in an underwater navigation solution. This approach is necessitated and further complicated by the operational paradigm-short-range vehicle operations in an under-ice environment and in an unpredictable sound speed feature, the Beaufort Lens. ${ }^{1}$ The Beaufort Lens favors long-range transmissions in its subsurface duct and creates a shadow zone for short-range transmissions at an AUV's operational depths.

An anecdote nicely portrays how the approach was successful in achieving GPS-like navigation for the vehicle deployment. ${ }^{2}$ The vehicle did not engage in its "return to home" behavior because a harddrive error aborted the mission. At that time, the vehicle stalled on the surface, underneath the ice, and broadcasted its position estimate. The team that went to retrieve the vehicle, tens of hours later due to a storm, dug a hole in the ice and found the vehicle precisely where it said it would be. Needless to say, due to the time-intensive nature of digging a hole

\footnotetext{
${ }^{1}$ Poulsen et al., "Acoustic noise properties in the rapidly changing Arctic Ocean"; Schmidt et al., "Acoustic Communication and Navigation in the New Arctic - A Model Case for Environmental Adaptation”.

${ }^{2}$ Randeni et al., "A high-resolution AUV navigation framework with integrated communication and tracking for under-ice deployments".
} 
through a couple meters of ice, a position error greater than tens of meters would have been enough to severely delay or hinder recovering the vehicle, even with a diver or ROV handy.

This chapter analyzes the range estimation from modem events between GPS-tracked buoys, which provides a reliable ground truth to validate the Virtual Ocean and the embedded acoustic processing. The experiment design is the gold standard for underwater acoustic navigation, as much of the literature casts uncertainty as the error between different localization methods and/or performs the localization efforts in post-processing with no GPS or DVL ground truth to compare to. In this work, we clearly delineate which results stem from real-time and postprocessed results; importantly, all proposed pipelines can be easily integrated into the existing fieldwork software architecture.

This chapter begins with a background about the ICEX20 navigation solution and a discussion of the modem experiment design, drawbacks, and assumptions. Two methods to predict the group velocity are proposed; one is used during ICEX20 and the other is an improvement based on the recorded data and developed in post-processing. The results are organized by an analysis of real-time range estimates; a comparison between group velocity prediction methods for the real-time data; an extended comparison for all beacon to beacon events to evaluate the improvement in methodology.

\subsection{INTEGRATED COMMUNICATION AND NAVIGATION NETWORK}

Acoustic navigation solutions and other vehicle operations in the Arctic are reviewed in Chapter 2. This section briefly covers the proposed navigation solution for ICEX20, the Integrated Communication and Navigation Network (ICNN). ${ }^{3}$

\footnotetext{
${ }^{3}$ Randeni et al., "Construction of a high-resolution under-ice AUV navigation framework using a multidisciplinary virtual environment”.
} 
The ICNN was initially developed via numerous virtual experiments, to push the boundaries of algorithms and interfaces between different hardware components. The simulation approach is a necessary testbed for robustness and produces better results than post-processing previous field data, as that restricts mission configurations to the data taken, which can hamper troubleshooting in the field. Here the simulation capabilities are largely physics-driven with a modular system of systems approach: an environmental simulator with sub-components for the ocean, Arctic ice, and ocean acoustics; a vehicle simulator with sub-components for vehicle dynamics and navigation; a topside hardware simulator and acoustic communications simulator, both with a software and hardware-in-the-loop version. ${ }^{4}$ The virtual environment similarly emulates the interfaces between the real components to test the entire software pipeline. Both simulation capabilities are integral to mission success.

The ICNN is comprised of four ice buoys, loosely in a rectangle, roughly $2 \mathrm{~km}$ away from a central ice camp with a topside computer. Each ice buoy is outfitted with a WHOI MicroModem, ${ }^{5}$ with a four-element receiver array and a single transmitter. The receiving and transmitting elements were split between shallow and deep depths (30 and $90 \mathrm{~m}$, respectively) to provide better coverage due to the acoustic shadow zone in the Beaufort Lens. The buoys do not encompass the full operating range of the vehicle but are positioned to minimize overlap in trilateration for spherical positioning. ${ }^{6}$

Vehicle missions in any underwater environment, but especially acoustically complex ones, must balance competing uses of the acoustic channel. Thus this network uses a single synchro-

\footnotetext{
${ }^{4}$ Schneider et al., "NETSIM: A Realtime Virtual Ocean Hardware-in-the-loop Acoustic Modem Network Simulator".

${ }^{5}$ Singh et al., "Underwater Acoustic Navigation with the WHOI Micro-Modem".

${ }^{6}$ Deffenbaugh et al., "The relationship between spherical and hyperbolic positioning".
} 
nized digital communication packet to provide both tracking and data to the operator. The ICNN operational paradigm is as follows:

1. The AUV, running an ice-tracking DVL and an onboard hydrodynamic model, broadcasts its perceived location on a scheduled, time-synchronized message via WHOI MicroModem

2. Four ice buoys, each outfitted with a WHOI Micro-Modem, receive messages from the AUV and send that information over freewave radio to a Topside computer

3. The topside computer converts travel times into range estimates using a stochastic embedded prediction of the horizontal group velocity via BELLHOP ray tracing code ${ }^{7}$

4. The topside computer calculates a new position by trilaterating the range estimates

5. The position differential is broadcast to the vehicle to update its navigation solution

The work in this thesis specifically addresses the third step-the topside computer's conversion of travel time into range, which underpins the trilateration in the ICNN, and is an often overlooked element of any underwater acoustic navigation system. The results in this chapter, accordingly, focuses on validating acoustic range estimates from communication events between GPS-tracked beacons. This "modem experiment" was conducted while the vehicle was still being prepped to go under the ice.

\footnotetext{
${ }^{7}$ Porter, The BELLHOP Manual and User's Guide: Preliminary Draft.
} 


\subsection{MODEM EXPERIMENT DESIGN}

Because the navigation solution on the vehicle, during a mission, can only be evaluated on the basis of the error estimates sent, a sister experiment for validating the real-time ranging approach was implemented. The ice buoy modems, at a given source depth, were run as "virtual vehicles" and would transmit to all other ice buoys. The internal sound speed estimate was then toggled between "baseline" mode, i.e. the depth-dependent mean, and a "weighted" mode, i.e. the chosen EOF weights. This trial was configured for as many ice buoy modems as possible, with each experiment running for roughly an hour between the two sound speed modalities. There are three important engineering choices in the ICNN network to contextualize this dataset:

1. Each modem buoy only has one transmit depth layer $(30$ or $90 \mathrm{~m})$

2. The transmit and receive layers are independent

3. At any given time, the receive depth layers are the same across all four ice buoys

The design here enables self-adapting network to transmit and receive at the optimal depth to maintain contact with the AUV. ${ }^{8}$ This adaptivity was not used during the modem experiment but was toggled manually. Given the complexity of the ICNN system, this experiment did not collect an exhaustive set of data across all buoy, source depth, receive layer, and sound speed combinations, as this time was also used to verify and resolve any hardware issues in the modems themselves.

Importantly, only one modem at a time could run the vehicle behavior to trigger group velocity calculations; other modems that were transmitting simultaneously recorded events as modems, not as vehicles. This work, which builds on the TDA framework, assumes the range independent

\footnotetext{
${ }^{8}$ Schneider et al., "Self-Adapting Under-Ice Integrated Communications and Navigation Network".
} 
propagation environment and organizes the acoustic events by the source depth and sound speed profile. This is a valid assumption given that source depth, as opposed to temporal or spatial scales or variability, is the dominating factor for the location and span of the shadow zone.

The dataset introduced in this thesis has parallel physical and virtual layers; given the small operational scale, on the order of kilometers, and a singular SSP, both layers are projected into a range independent coordinate system for ease of comparison. The physical layer describes the beacon to beacon communication events through the real water column; it covers all source depths $(20,30$, and $90 \mathrm{~m})$ and both receive layers (30 and $90 \mathrm{~m})$, where the surface expressions of the beacons are tracked by GPS. The virtual layer describes the BELLHOP simulations for each communication event in the physical layer and toggles between two sound speed environments, the depth dependent mean and the chosen EOF weights. The SSP, along with source and receiver information, are the only manually configurable inputs to the group velocity prediction. The group velocity prediction drives the range estimation component of vehicle trilateration.

\subsubsection{Spatial AND informational OVERVIEW}

Subfigure 5.1a shows a chart for the successful modem connections based on source depth. This chart introduces color and symbol coding that is maintained for many figures analyzing beacon configuration. It is organized by transmit (tx) on the left and receive (rx) on the right. The sideways pointing triangle indicates a transponder at $20 \mathrm{~m}$; the upward pointing triangle indicates the shallow transponder at $30 \mathrm{~m}$; a downwards pointing triangle indicates the deeper transponder at $90 \mathrm{~m}$. The "North" and "South" ice buoys are indicated with red and orange, respectively; the "West" and "East" ice buoys are indicated with green and blue, respectively. The centrally located topside "Camp" is notated in purple. 


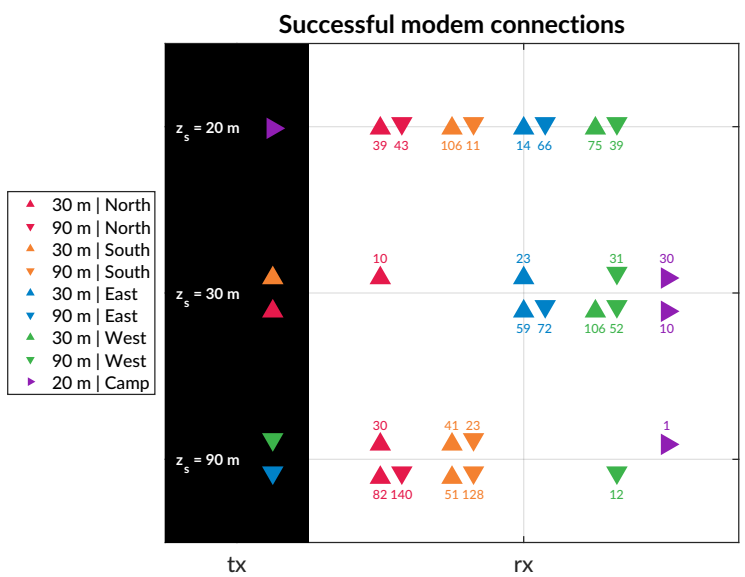

(a) A chart of the successful events between source and receiver depth. The black column on the left, $t x$, shows the source depth, $z_{s}$. The column on the right, $r x$, shows the receivers with the amount of good contacts. The orientation of the triangles-sideways, upwards, and downwards-corresponds to depths of 20,30, and $90 \mathrm{~m}$.

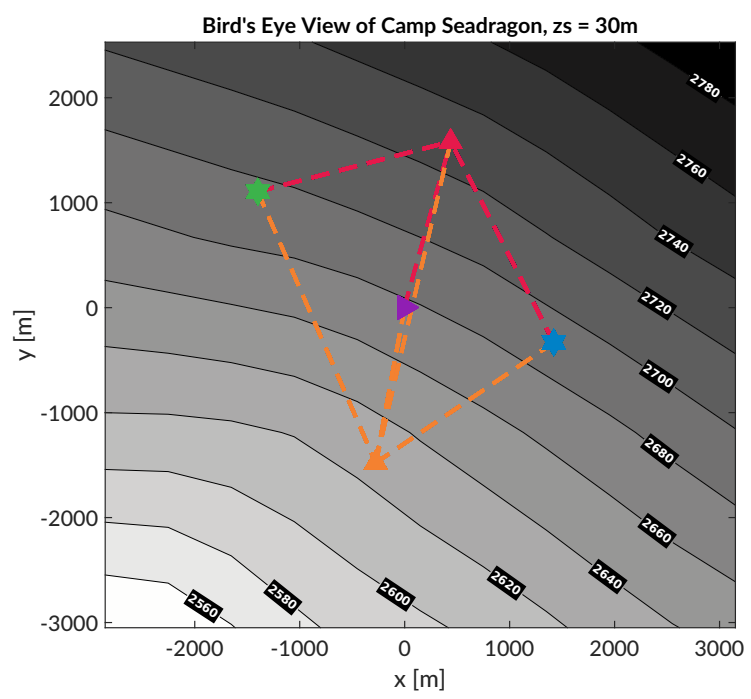

(c) The contacts for a source depth of 30 meters; the dashed line indicates paths taken from the source.

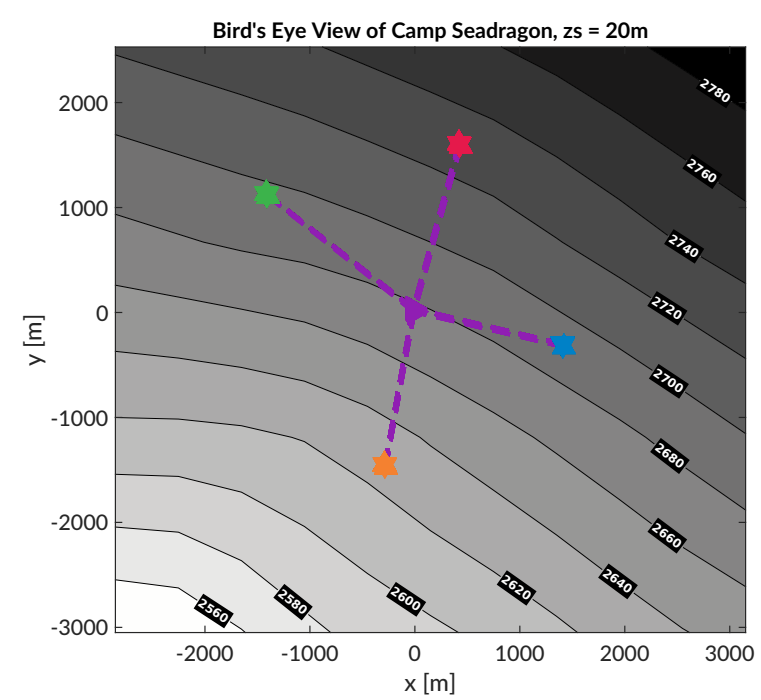

(b) The contacts for a source depth of 20 meters; the dashed line indicates paths taken from the source.

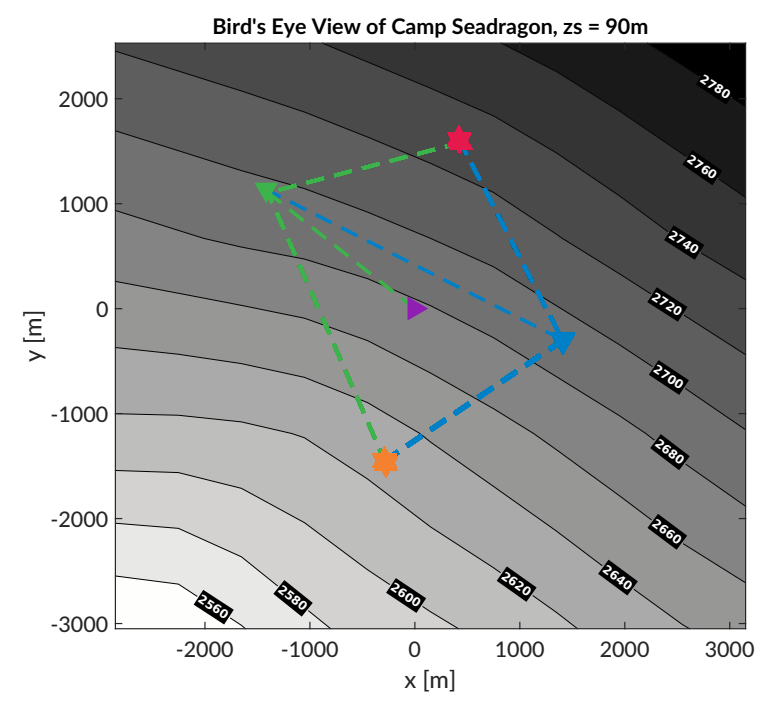

(d) The contacts for a source depth of 90 meters; the dashed line indicates paths taken from the source.

Figure 5.1: An overview of the modem experiment by source and receiver depth and position. Subfigures (b-d) correspond to receiver depths of 20, 30, and 90 m, respectively. Camp Seadragon, demarcated by the purple triangle, is situated just north of the continental shelf. 
The numbers above or below each receiving icon indicate the number of successful modem connections for that source-receiver pair. Because of the experiment design, these numbers should not be compared as a metric for the intermittency of the acoustic channel. Similarly, the exact source-receiver pairing, while useful context, is indicative of experimental constraints in ICEX20, not any spatial or temporal variability that drastically modified propagation. There are other physical features, like ice keels or pressure ridges, that could have played a role in preventing propagation; quantifying their exact impact is beyond the scope of the available or collected data. Because we assume range independent acoustic propagation, downstream analysis will ignore the lateral positioning of the beacons themselves and operate in a planar coordinate system.

The rest of figure 5.1 shows the successful modem connections from each source depth over a bathymetric view of Camp Seadragon. The large majority (more than 99\%) of acoustic events do not interact with the bottom, further simplifying the range independent propagation. Topside (in panel 5.1b) is the only source at $20 \mathrm{~m}$; it is received by all ice buoys at both shallow and deep receivers, as indicated by the purple lines emanating from the topside icon. North and South transmitted at $30 \mathrm{~m}$ source depth, as shown in panel 5.1c. Received acoustic events, in this case, are not perfectly mirrored between the two sources. North (red) only transmits to Camp, East, and West, whereas South (orange) also transmits to North. West and East transmitted at $90 \mathrm{~m}$ source depth, as shown in panel 5.1d. West (green) transmits to North, South, and Camp, whereas East (blue) transmits to North, South, and West.

\subsubsection{Timeline of COMmUniCATION, SOUND SPEEd ESTIMATES, AND ICE DRIFT}

Figure 5.2 demonstrates the source (top) and receiver (bottom) connections with respect to the sound speed configuration, where the base value is the white region and the EOF value is in the 
shaded region. These sound speeds are demonstrated in figure 5.3. An additional sound speed, from HYCOM, is shown here to demonstrate model mismatch. The HYCOM sound speed is injecting into the pipeline for post-processing results as well.

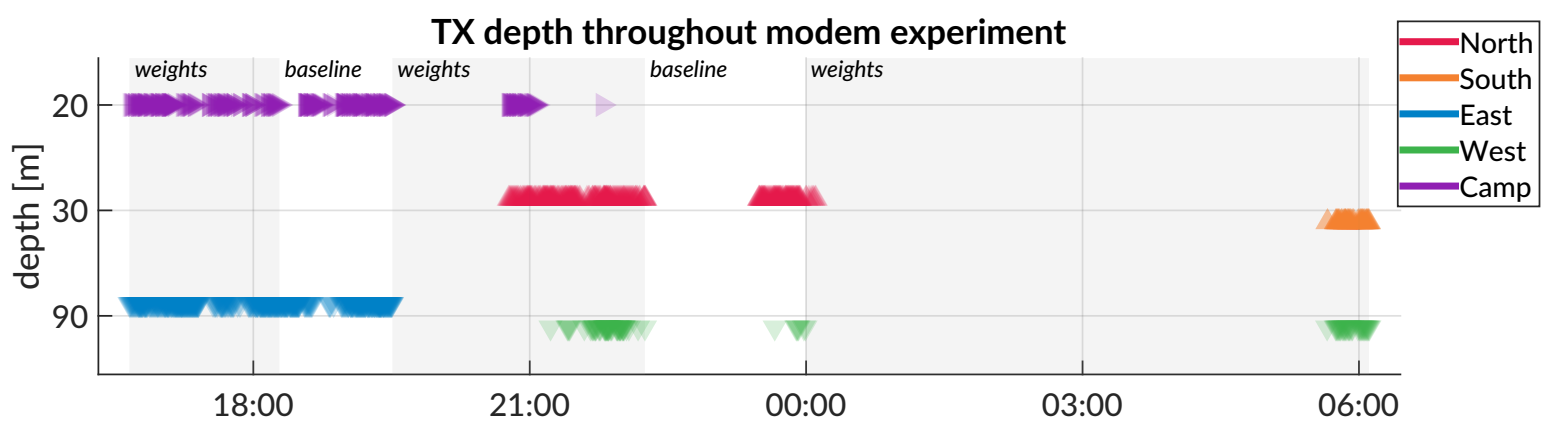

RX depth throughout modem experiment

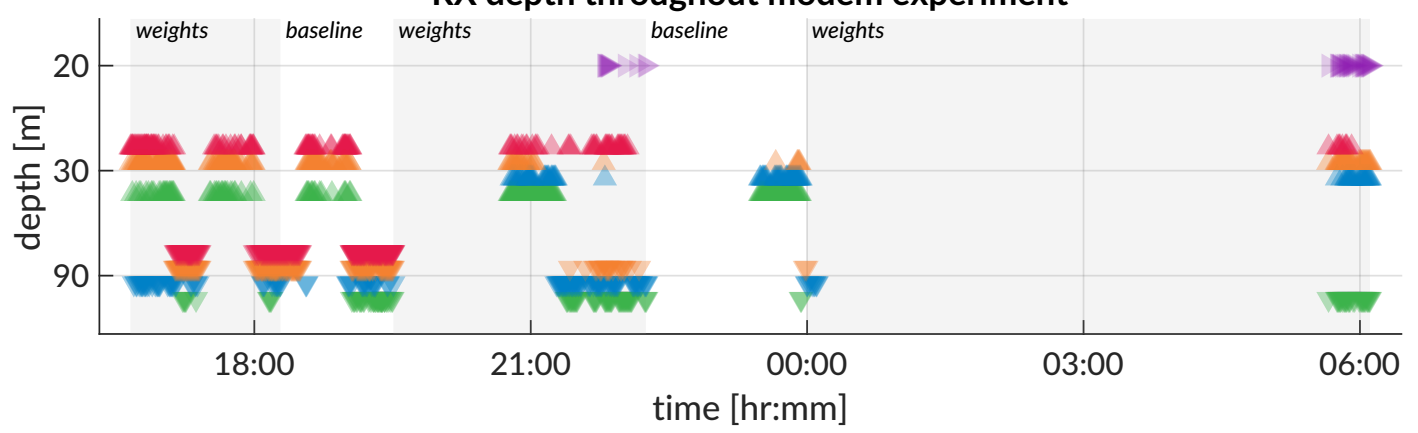

Figure 5.2: A timeline of source and receiver depth throughout the modem experiment. The modem colors \& shapes are indicated by figure 5.1a. The transponder depths are at the nominal depths; their offset from the horizontal is for spacing on the figure. The "baseline" and "weights" indicate which sound speed estimate was running in the Virtual Ocean.

To save time, multiple sources were transmitting in the same configuration but at offset synchronized schedules. This concomitance also contributes to the perceived discrepancies in reciprocity for source-receiver pairs. It also illustrates a shortcoming of this dataset, in that more events are collected with the weighted sound speed running in the Virtual Ocean as opposed to the baseline. This of course has no bearing on the successful receipt through the water column.

The acoustic ranging estimate between beacons is compared to their GPS locations as tracked by the surface buoys. As the experiment sits atop an ice floe, the ice can and does move relative 


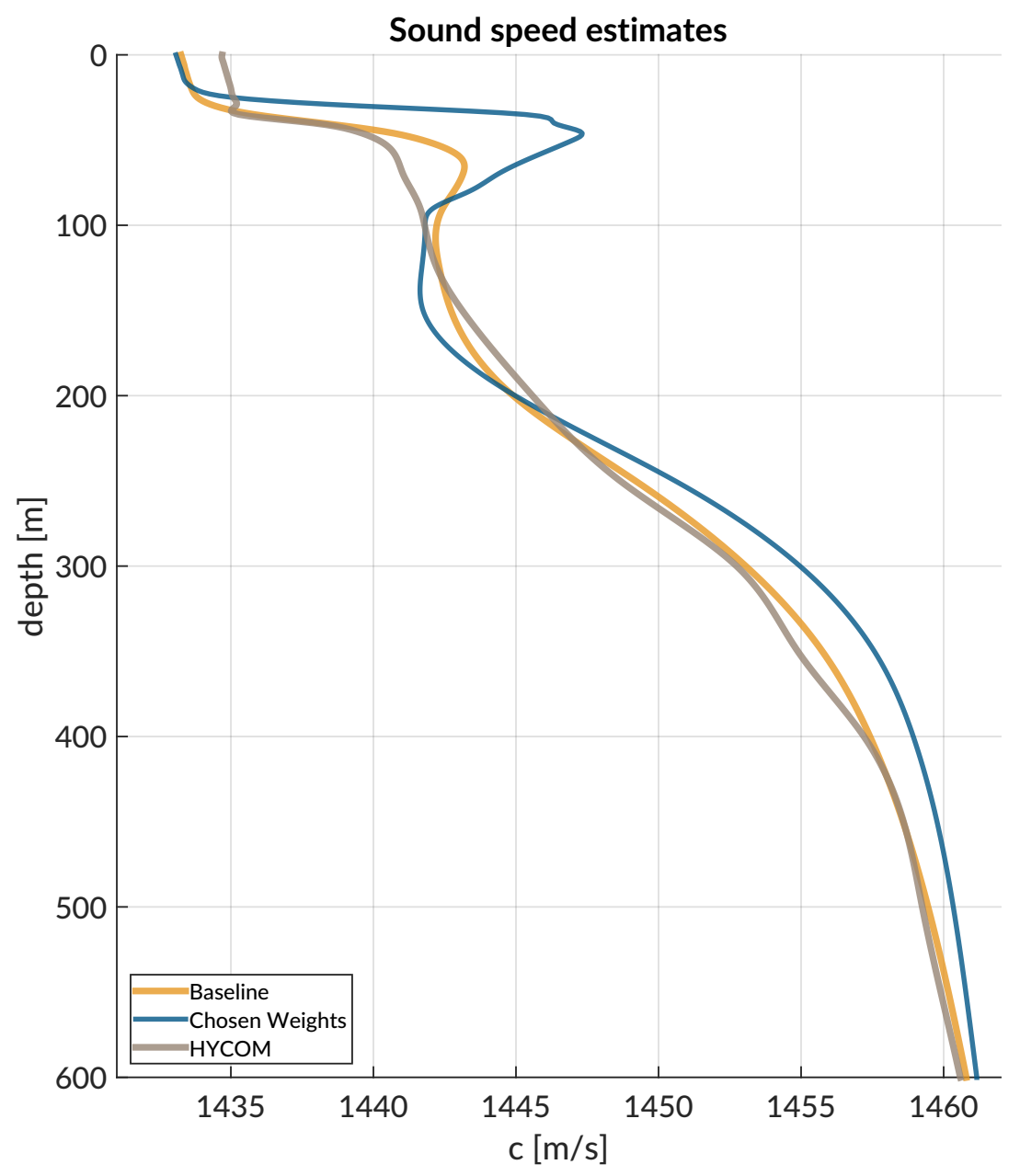

Figure 5.3: Sound speed estimates for range estimation; the baseline (mean of the EOF set) and chosen weights (weights chosen by LT Howard) are used in situ for ICEX20; the HYCOM (gray) is only run in post-processing as a natural comparison.

to some absolute coordinate system, as shown in figure 5.4. The ICNN takes this relative motion into account when updating vehicle position. Positioning of the elements ignores any displacement on the cable hosting the modems itself, as this is assumed to be negligible for such short cable distances. Throughout the entirety of the beacon to beacon experiment, roughly 12 hours, the ice floe moves almost $40 \mathrm{~m}$ relative to where it started. The majority of that movement happens during a short span of time, roughly 3 hours, further indicating the necessity for taking into 


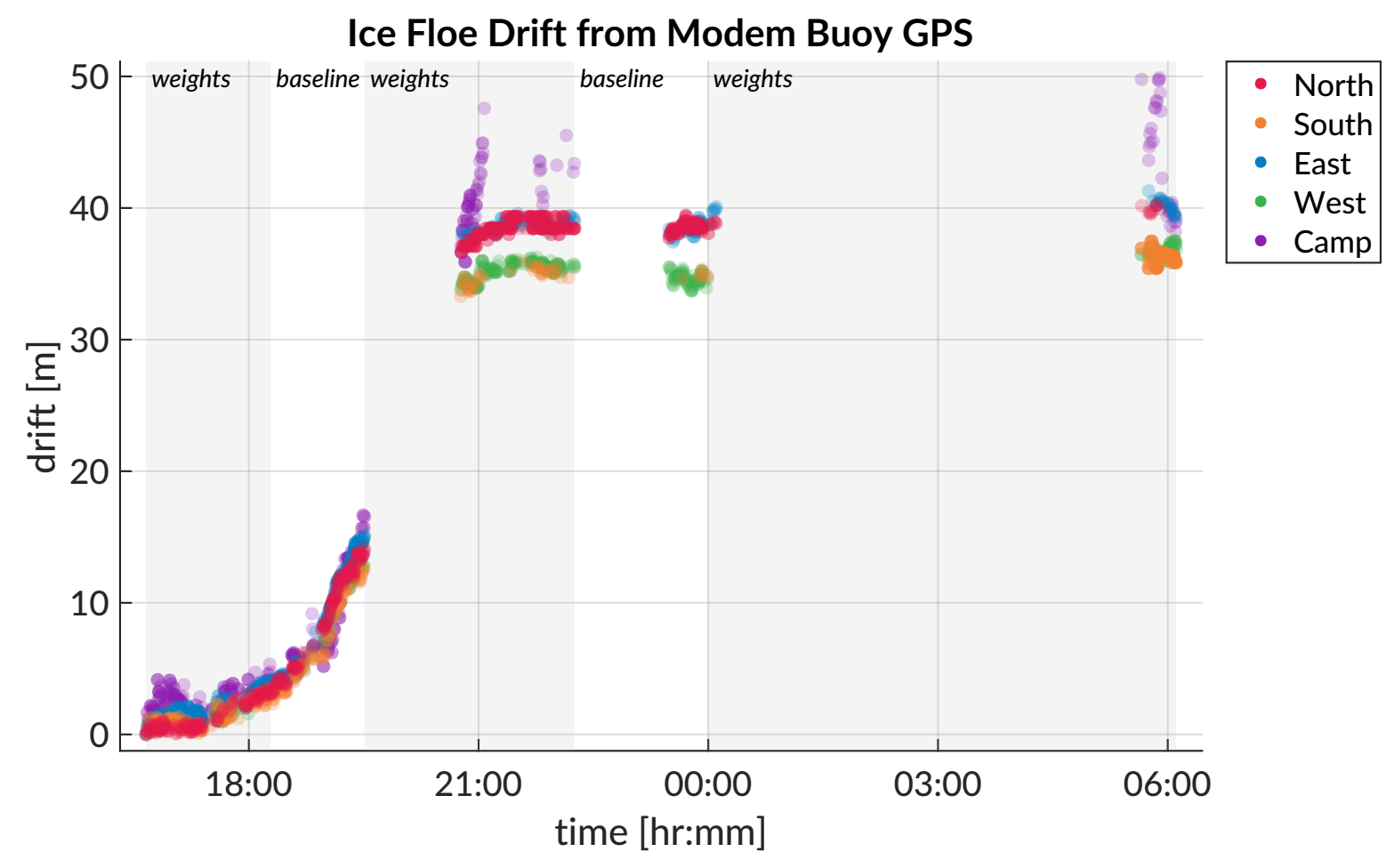

Figure 5.4: The ICNN accounts for ice drift throughout the mission duration. This is the magnitude of the ice drift recorded, at each buoy, throughout the modem experiment. The shaded regions reference which sound speed estimate was used, as indicated in figure 5.2.

account ice movement for vehicle navigation. Notably the topside GPS experiences less precision than the other ones, likely due to the amount of activity and infrastructure around the GPS puck itself. This work does not correct the GPS estimate, but it is of minimal consequence given that most events do not include the Camp beacon, and that the drift is less than $1 \%$ of the range from Camp to any other beacon. The analysis in this work removes communication events with travel times greater than 4 seconds, as these would indicate rare bottom bounces that the ICNN filters as outliers. This threshold is informed by a statistically moving average, our operational scales, and the upward refracting and ducted acoustic propagation environment of the Beaufort Lens, where it is much more likely to receive surface interacting paths than bottom interacting ones. 


\subsubsection{RANGE INDEPENDENT ASSUMPTION}

Accounting for ice movement creates nominal ranges with small variability for range independent propagation. Figures 5.5, 5.6, and 5.6 show the eigenrays for beacon to beacon events for the three SSPs for nominal source depths of 20,30, and $90 \mathrm{~m}$, respectively. The eigenrays are found using BELLHOP built-in eigenray protocol with a launch angle step of 0.05 degres from 60 and 60 degrees. It is important to note that the built-in protocol uses a nominal capture radius to classify a ray as an eigenray, i.e., it is not an exact solution. In general, BELLHOP is a greedy estimator and will return many eigenrays for any source-receiver pair. For these calls, it returns anywhere from 10 to 40 eigenrays; of those, one eigenray is selected as the nearest from travel time proximity to the recorded data, as that is the important variable for range estimation. The travel times were organized by clustering with 1 millisecond boundaries such that some sourcereceiver pairs have multiple, distinct travel times to fit. Thus, a reasonable hypothesis for these diagrams is that the eigenrays will converge to the receiver locations for the most realistic sound speed input (unfortunately, this is not exactly what happens).

Figure 5.5 shows the eigenrays for a source depth of $20 \mathrm{~m}$. All modem buoys receive at their shallow and deeper nodes at ranges from 1.5 to $1.8 \mathrm{~km}$. For this source depth, reliable eigenrays are found for each sound speed condition. Rays refract upwards and intersect neatly with all receiver locations. The ray paths for the upper receivers, notably, change both in the number of surface interactions and where the surface interactions occur with respect to range across the different SSPs. In one case, as the SSP becomes more ducted, the chosen paths for the Northern (red) receiver interact with the surface more and become distinct paths. The ray paths for the lower receivers lose direct path in the weighted SSP compared to the other two. In addition, we hit a computational limit as the eigenrays for the Northern (red) and Western (green) buoys at 90 
$\mathrm{m}$ are visually the same ray, even though neither is "optimal" compared to their counterparts in other SSPs.
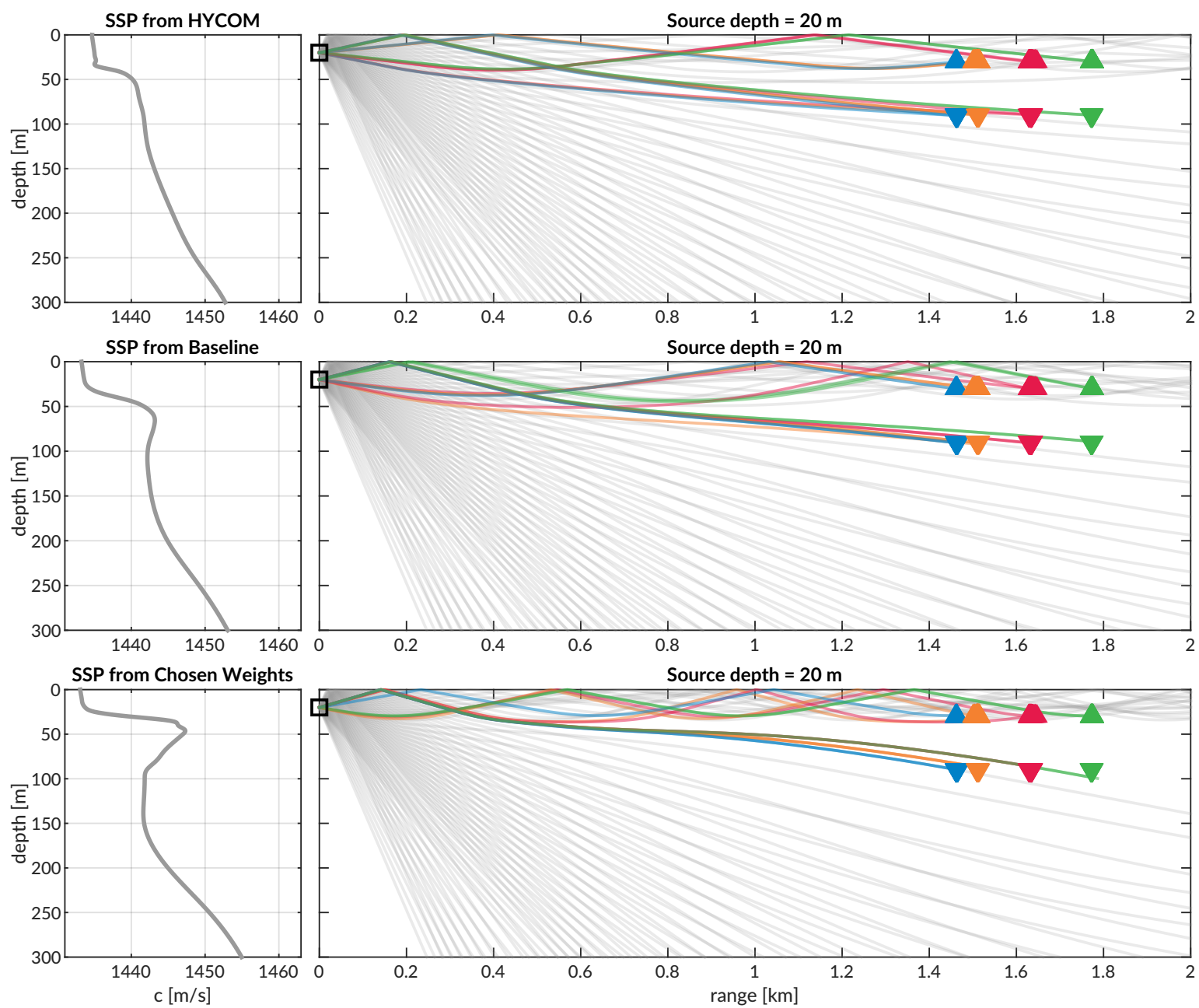

Figure 5.5: Eigenrays for beacon to beacon events for the nominal source depth of $20 \mathrm{~m}$. The beacons are highlighted in color/marker coding in figure 5.1a, and the eigenrays from BELLHOP are traced in the representative colors over the ray fan in gray.

Figure 5.6 shows the eigenrays for a source depth of $30 \mathrm{~m}$. Because North and South are the source locations, the receiver locations are spread out from 1.5 to 3.2 kilometers. BELLHOP is fairly consistent in finding reliable acoustic paths to the receivers, though it struggles in the extreme ducted conditions brought on by the weighted SSP. And in a similar vein to those from 
a $20 \mathrm{~m}$ source depth, the reported eigenrays demonstrate increased surface interactions. What is most notable about these ray sketches is the dominating shadow zone that begins at $2 \mathrm{~km}$ in range and the similar collapsing of eigenrays in approximating reliable acoustic paths to lower receivers. Computationally, this means that BELLHOP is not finding rays that enter that region. One solution would be to increase the density of the ray fun, but this is computationally intensive. Those connections may rely on minor spatio-temporal variability that can never be perfectly resolved in a simulation.

Lastly, figure 5.7 shows the eigenrays for a source depth of $90 \mathrm{~m}$. Because East and West are the source locations, the receiver locations are similar to that of the source depth of $30 \mathrm{~m}$, from 1.5 to 3.2 kilometers. The deeper source depth effectively negates the upper duct and places the upper (and some of the lower) receivers in a shadow zone. The HYCOM eigenrays still show the most reliable acoustic paths; these deteriorate slightly with the baseline environment and drastically with the weighted one. Notably, there are no direct paths in the weighted SSP, whereas direct paths are found in the HYCOM and baseline SSPs.

The analysis here demonstrates a troubling trend for model-aided (BELLHOP) prediction of the group velocity in extremely ducted conditions - the more realistic sound speed input can push computational limits in finding exact eigenrays. By all accounts, HYCOM seems like the most promising (and accurate) sound speed environment given the travel times observed, even though the locally observed SSP is the weighted one. This visual accuracy would likely deteriorate for longer range propagations.

Generally, the visual fit for the eigenrays deteriorates with the ducted environments in the baseline and weighted SSPs. Part of what complicates the realism in the acoustic model is how the steep sound speed slope above the lens and the numerical resolution as the ray passes through 

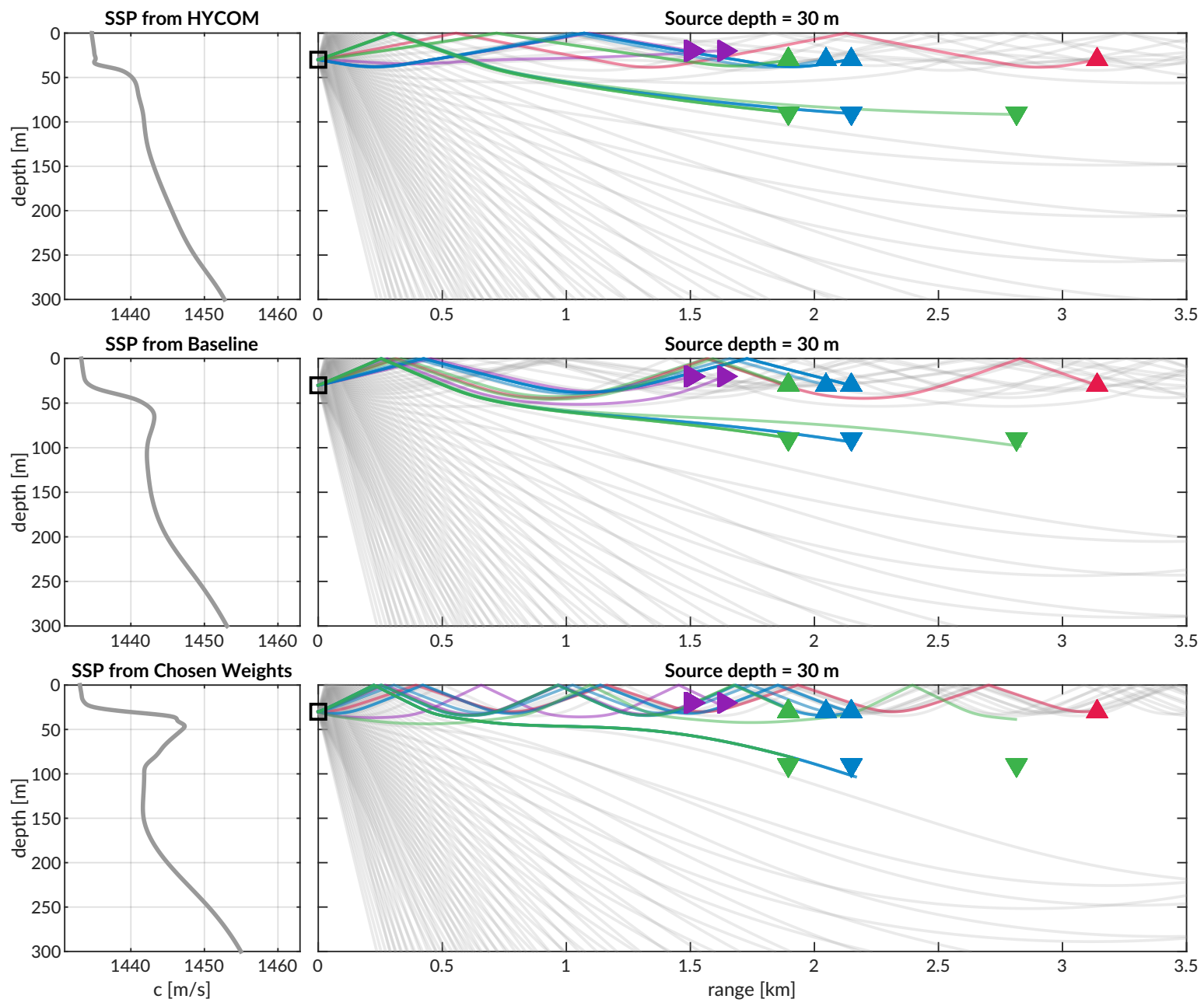

Figure 5.6: Eigenrays for beacon to beacon events for each nominal source depth of $30 \mathrm{~m}$. The beacons are highlighted in color/marker coding in figure 5.1a, and the eigenrays from BELLHOP are traced in the representative colors over the ray fan in gray.

it. Discrepancies in ray paths for the bottom receivers in particular are driven by the shadow zone created by strong ducting.

One caveat in this result is that the eigenrays are sought after for a point location, i.e., the GPSderived range between beacons and the nominal depths of those beacons, for a precise travel time. This precision in finding rays between a point source at an exact depth and a point receiver at an exact depth, an exact distance apart, pushes the computational limits BELLHOP provides in an 

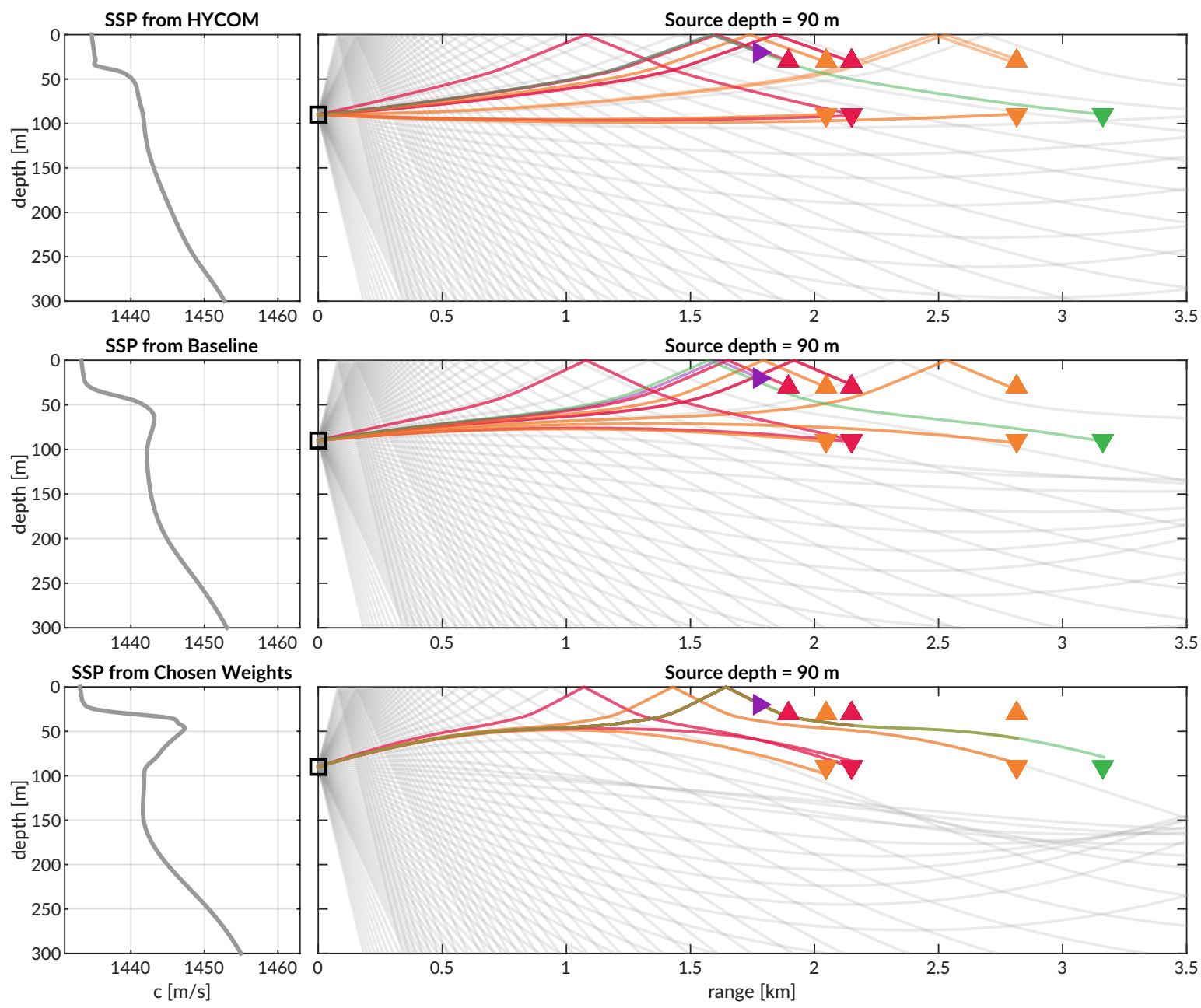

Figure 5.7: Eigenrays for beacon to beacon events for each nominal source depth of $90 \mathrm{~m}$. The beacons are highlighted in color/marker coding in figure 5.1a, and the eigenrays from BELLHOP are traced in the representative colors over the ray fan in gray.

automated fashion. While it is possible to increase the density of launch angles, or implement a recursive search to find eigenrays, both of these methods burn through computational time and memory without guaranteed results. In this work, we aim to leverage BELLHOP simulations to accurately estimate the group velocity for a reliable transmission path between any given sourcereceiver pair. Even though BELLHOP may struggle with an extremely ducted environment, it is a greedy estimator of eigenrays and therefore provides great insight into various multipath 
propagation between a source and a receiver. Thus, we consider this computational constraint and exploit the greedy estimator by creating a local grid to solve for around a nominally exact range and depth, to add much needed redundancy in resolving the actual multipath structure and reliable acoustic paths. This grid also addresses the intermittency of vehicle updates (every thirty seconds) and the fact that the vehicle is moving in between updates.

\subsection{IN SITU GROUP VELOCITY ESTIMATION VIA MINIMAL BOUNCE}

\section{CRITERIA}

The variation of group speed from any source-receiver pair is the fundamental challenge to implement GNSS-like navigation in an LBL navigation paradigm, especially in an acoustically complex propagation environment. The use of the acoustic modem network for tracking relies on the accurate estimates of travel times between the submerged platform and range modems, supported by clock synchronization and a pre-determined scheduling of acoustic events. For the Beaufort Lens, the strong multipath effects make it virtually impossible to deterministically predict the modem triggering time. Instead, for each individual node $i$, an embedded stochastic tracking framework is used to provide a running estimate of the horizontal group velocity $u_{i, j}$ for the conversion from travel time to range from modem $j$,

$$
r_{i, j}=u_{i, j} \Delta t_{i, j}
$$

In the ICEX20 configuration, the acoustic tracking is running on the topside computer, which controls the integrated communication and navigation range. Here we assume that the group velocities $u_{i, j}$ are smoothly varying over the course of a vehicle mission, i.e., with respect to range, 
mission time, and the frequency of updates relative to vehicle motion. The group velocity is continuously tracked using predictions from the Virtual Ocean infrastructure.

When the topside tracking framework receives a modem message, with a time delay, $\Delta t$, from one of the range modems, it will request a new estimate of the group velocity and its associated uncertainty. The group velocity estimate is found using the extrapolated navigation for range, $\hat{r}$, and an impulse response estimate received in the form of ray travel times $d t_{j}$ and amplitudes $a_{j}$ for that range and depth.

The initial call to BELLHOP is over a sparse, dynamically created depth and range grid, of which the plane wave approximation is used to bring the ray to the range and depth currently posited by the onboard tracking solution. Using only the $N_{0}$ rays with neither surface nor bottom bounces, it will then estimate the current group velocity $u$ from a power weighted average of the ray travel times,

$$
u=\frac{\hat{r} \sum_{n=1}^{N_{0}} a_{n}^{2}}{\sum_{n=1}^{N_{0}} d t_{n} a_{n}^{2}},
$$

and the associated weighted standard deviation,

$$
\sigma_{u} \simeq \sqrt{\frac{\sum_{n=1}^{N_{0}}\left(d t_{n}-\hat{r} / u\right)^{2} a_{n}^{2}}{\sum_{n=1}^{N_{0}} a_{n}^{2}}} \frac{u^{2}}{\hat{r}}
$$

If no direct paths exist, i.e. $N_{0}=0$, then the group velocity is computed using the same algorithm for the ray arrivals with one bounce, and so on.

This stochastic method for group velocity calculation can run in real-time, appearing to be orders of magnitude faster than general post-processing methods which seek to determine the specific ray itself that best matches a prominent indicator from the arrival structure. The method assumes direct path arrival, where multipath over the shallow receiver depth is trivial for the 
group velocity calculation; thus we call it the minimal bounce criteria (MBC). The BELLHOP simulation that runs this calculation uses 3600 rays with launch angle fan of -60 to 60 degrees. However, this high density of launch angles creates a greedy estimator of direct path eigenrays, and this simulation pipeline almost always guarantees a direct path found. The MBC improves upon a deterministic estimate in that it considers the ray path, travel time, and amplitude to predict a realistic group velocity.
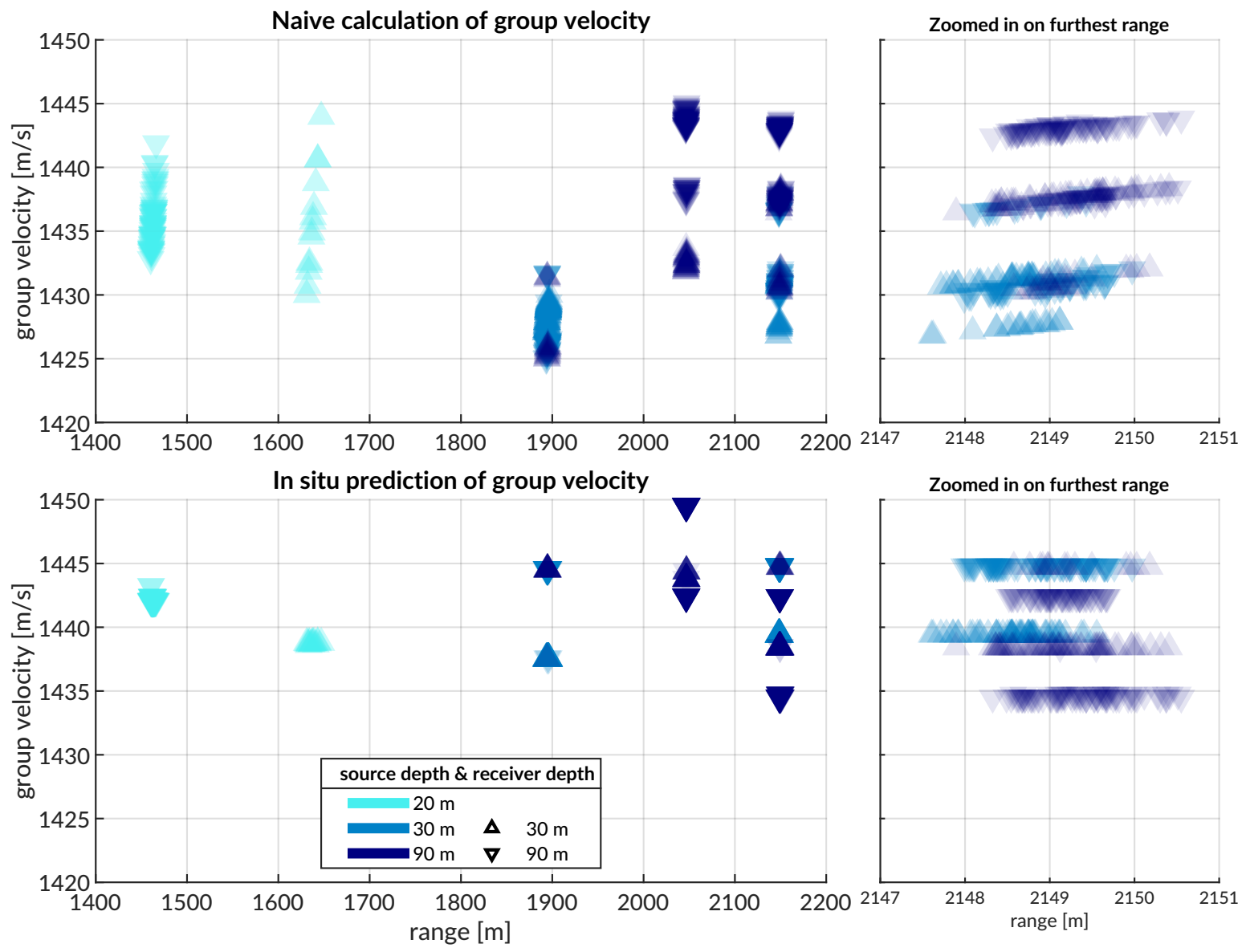

Figure 5.8: A comparison of the naive and in situ simulated group velocity. The naive group velocity is simply the distance between two nodes, recorded by GPS, divided by the OWTT between them. The simulated group velocity was estimated in situ during ICEX20. The panel on the right zooms in on a subset of range to show the spreading in group velocity predictions. 
A comparison of the simulated and the naive group velocity for all modem events is shown in figure 5.8. The naive group velocity is simply the GPS-recorded distance between two nodes divided by the OWTT between them. The simulated group velocities via MBC exhibit a slightly narrower range (1434 to $1450 \mathrm{~m} / \mathrm{s}$ ) compared to the naive ones (1425 to $1445 \mathrm{~m} / \mathrm{s}$ ). The simulated process overestimates the naive group velocity because it assumes a direct arrival. The color and symbol encoding show source location and depth. The naive group velocity shows small spreads for the same nominal ranges, whereas the prediction does not; the spreading here is most likely driven by GPS sensitivity to ice movement and GPS drift. Both sets shows strong clusters in the group velocity by source and receiver depth pairings, driven by the various ray paths taken between the source and receiver pairings. The group velocity, which we originally assume to be smoothly varying with respect to range, is sensitive to relatively small differences in source and receiver depth as well. The standard deviation of the group velocity is no more than $0.5 \mathrm{~m} / \mathrm{s}$ for all recorded events.

The inset, on the right, zooms in on the naive and predicted group velocity structure for the furthest range with several data, 2147 to $2151 \mathrm{~m}$. The naive calculation shows a positive correlation of range and group velocity with approximately a slope of 1 ; this would either indicate that the ice is moving non-rigidly between the modem buoys or that the naive group velocity calculation is dominated by GPS drift with minimal OWTT drift. Because the simulated prediction shows no group velocity changes with respect to local changes in range, this is more likely driven by GPS drift than ice movement. Given the patchwork of discrete ranges from the source, the relatively small and tight clusters corroborate the driving assumption that the group velocity is slowly varying in range for a given source depth. 


\subsection{NEW GROUP VELOCITY ESTIMATION VIA NEAREST BOUNCE}

\section{CRITERIA}

As shown in the eigenray traces, an acoustic arrival does not always take the direct path from source to the receiver, and the path it takes is extremely dependent on the sound speed profile. Often, BELLHOP will produce a number of arrivals resulting from multiple propagation paths for any source-receiver pair. The suggested algorithm, the nearest bounce criteria (NBC), is only a slight modification from the minimal bounce criteria, and includes multipath as a new dimension of information with negligible additional computation.

Given a running estimate for the horizontal group velocity $u_{i, j}$ between nodes $i$ and $j$, the navigation system has an extrapolated value for range, $\hat{r}$, in the virtual layer, and a recorded travel time, $\Delta t_{i, j}$, in the physical layer. Instead of using only the $N_{0}$ rays with neither surface nor bottom bounces to estimate group velocity, we solve for the power weighted average of the ray travel time for the $N_{k}$ rays with $k$ bounces,

$$
t_{k}=\frac{\sum_{n=1}^{N_{k}} d t_{n} a_{n}^{2}}{\sum_{n=1}^{N_{k}} a_{n}^{2}},
$$

find the nearest matching power weighted average to recorded travel time,

$$
t_{i, j, k}=\min _{k=0,1,2, \ldots}\left|t_{k}-\Delta t_{i, j}\right|
$$

predict a group velocity,

$$
u_{i, j}=\frac{\hat{r}}{t_{i, j, k}}
$$


and estimate the range as was done previously.

$$
r_{i, j}=u_{i, j} \Delta t_{i, j}
$$

This method selects a different group velocity based on the multipath arrival structure, as the expected arrival is not always the first arrival, and could be masked by noise or blocked temporarily. $^{9}$

In the post-processing method, we limit the number of bounces to 4 because of the smaller operational scale and the attenuation accrued with many surface interactions. Even with the Beaufort Lens duct, the Arctic SSP is still upward refracting, so the number of bounces here do not encode surface or bottom bounces separately. In addition, the grid is not exactly replicated due to the nested gridded nature of vehicle processing and those variables not being saved in the vehicle logs, but it is based off of the grid created for some in situ events: it is $11 x 11$ points over 10 $\mathrm{m}$ in range and $20 \mathrm{~m}$ in depth. The extra size in depth addresses the computational limitations of how time-bounded eigenrays seem to stack in the water column, as shown in figures 5.5, 5.6, and 5.7. Lastly, to populate the multipath criterion effectively, we abandon the plane-wave extension to the interpolated receiver location. More detailed results for how this method predicts the group velocity are shown in full post-processing pipeline analysis, in section 5.7.3.

\footnotetext{
${ }^{9}$ Deffenbaugh et al., "Acoustic positioning in a fading multipath environment".
} 


\subsection{IN SITU RANGE ESTIMATION RESULTS FROM MINIMAL BOUNCE}

\section{CRITERIA}

The modem experiment generated 811 beacon to beacon communication events with their own real-time group velocity predictions. The topside computer collects the OWTT and current range estimate from the vehicle, and predicts the group velocity for each transmission path. The new range estimate, or psuedorange, is the predicted group velocity multiplied by the recorded travel time. This psuedorange is then fed into the trilateration for localization.

This section calculates range error as the directional difference between the psuedorange and the GPS derived range. The analysis is separated by source depth, and where possible, receiver depth, as the group velocities across source and receiver depths may be different. Figure 5.9 demonstrates the range error for source depths of 20,30 , and $90 \mathrm{~m}$, respectively, comparing a purely data driven framework to the embedded stochastic framework for the two possible sound speed environments. It is important to note that much of this section, while determining the range estimation error, also contextualizes the asymmetry in the data collected and, by extension, the limits of comparison or conclusions that can be made.

\begin{tabular}{r|ccccc}
\hline SSP & Count & Mean [m] & Median [m] & STD [m] & Max [m] \\
\hline Baseline & 243 & 11.38 & 11.96 & 4.23 & 23.95 \\
Chosen weights & 568 & 11.36 & 11.40 & 8.12 & 26.0 \\
\hline
\end{tabular}

Table 5.1: The range estimation error for in situ events. The error here is presented as the absolute, not directional, error.

Table 5.1 breaks down the number of events, mean, median, standard deviation, and maximum absolute range error for the in situ calculations. The WHOI Micro-Modem has a temporal reso- 
lution of $1 / 10^{\text {th }}$ of a millisecond; accordingly, we show significant digits to centimeter accuracy. The mean and median for both sound speeds are similar on the order of centimeters; the chosen weights show a maximum greater than that of baseline, but it is from a OWTT event that has no comparison in the baseline data set. The overarching statistical results here show that both sound speed environments, given the smaller operational scales and shallow depths, are good enough to approximate the group velocity structure given a local grid instead of a point approximation. Of course, these statistics obscure the relationship between range error and range (or OWTT as the proxy for range).

Figure 5.9 shows the range error boundary, separated by source depth (rows) and receiver depth (columns) and colored by sound speed input, against recorded OWTT. Once again, there are minimal differences between the two sound speed inputs.

If there was an assumed deterministic group velocity, the range error would grow with OWTT proportionally to the difference between the estimated and actual value. Thus, an indication that the algorithm is adapting group velocity correctly is that range error does not strictly increase with OWTT. Where there is overlap in the physical layer, i.e. in OWTT, the range error driven by the two virtual layers is no more than a few meters. This is best demonstrated by all source and receiver depth pairings, except for a source at $30 \mathrm{~m}$ and a receiver at $90 \mathrm{~m}$, where the baseline is clearly visibly closer to no range error than the weighted environment. This may be driven by the prominence of the duct, but it is certainly occluded by the order of magnitude difference between the number of events in each virtual layer. As expected, the algorithm is generally overestimating range as it solves for the direct path. 
In situ range error by source $(20,30,90 \mathrm{~m})$ and receiver $(30,90 \mathrm{~m})$ depths

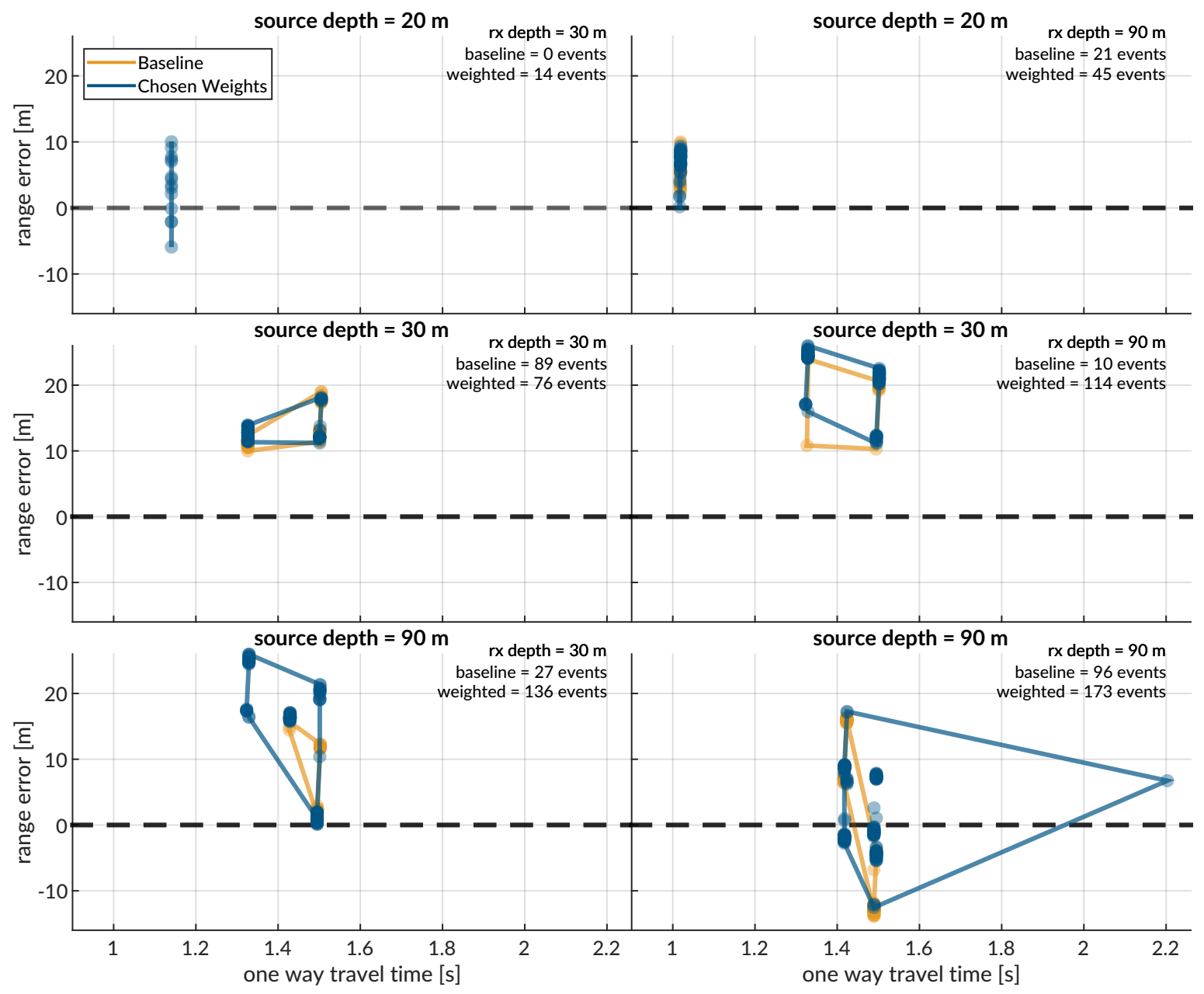

Figure 5.9: The in situ range error by source $(20,30$, and $90 \mathrm{~m})$ and receiver $(30$ and $90 \mathrm{~m})$ pairings for both sound speed estimates used during ICEX20. The amount of communication events that use each sound speed estimate is notated in the top right of each panel. The dashed line indicates no range error, and the boundary drawn indicates scope of the range error as a function of one way travel time.

\subsection{POST-PROCESSED RANGE ESTIMATION RESULTS FROM NEAREST}

\section{BOUNCE CRITERIA}

The nearest bounce group velocity prediction algorithm was proposed to mitigate the systemic overestimation driven by assuming the simplest multipath propagation and is evaluated in a post- 
processing pipeline. This section examines the range estimation via NBC for the 811 events with the in situ prediction. The post-processing simulation is seeded with the exact same extrapolated range, $\hat{r}$. Thus, this section analyzes how the virtual layer could have better matched the physical layer as a function of the group velocity prediction algorithms and the two sound speed estimates used in the field.

Figures 5.10 and 5.11 are information dense visualizations. Each event is coded by the source depth, in color, and the multipath structure, by marker symbol. The $\mathrm{x}$-axis refers to the in situ error using the minimal bounce criteria; the y-axis refers to the post-processed error using the nearest bounce criteria. Thus the shaded region indicates where the in situ performance is better than updated algorithm.

Figure 5.10 is a performance cross-map for the 243 events running the baseline SSP. Almost all events (99.18\%) lie in the white region, indicating that NBC better predicts the group velocity. The mean and median for the minimal versus nearest criteria are 11.38 and $11.96 \mathrm{~m}$ versus 2.61 and $1.58 \mathrm{~m}$, respectively. Furthermore, the updated algorithm does not have a systemic overestimation bias. The group velocity estimation sensitivity to not only range but source-receiver depth and multipath manifests as distinct clusters. One notable example of this is for a source depth of $30 \mathrm{~m}$, with an in situ error of $17-21 \mathrm{~m}$; there is a consistent offset between the error for what is perceived to be 1 and 2 bounces. Parallel clusters with the same color and marking indicate different propagation ranges.

A performance cross-map for the 568 events running the weighted SSP shows similar results. A majority of events (84.68\%) lie in the white region indicating improved performance. The mean and median for the minimal versus nearest criteria are 11.36 and 11.40 versus 4.53 and $2.42 \mathrm{~m}$, respectively. The NBC results are more biased to be positive than with the baseline sound speed, but show less overestimation than those of the MBC. The same behavior with clusters exist, and 


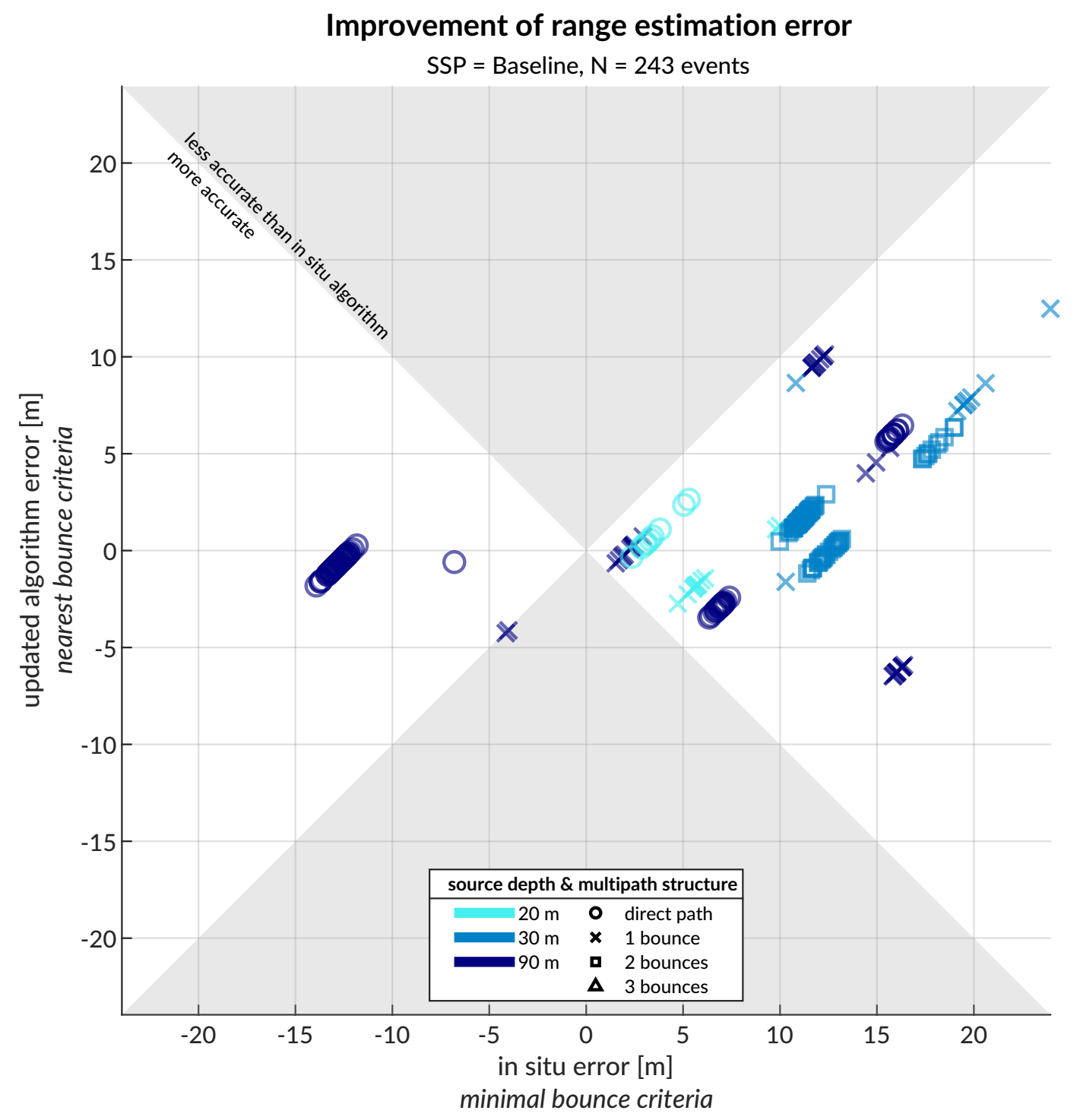

Figure 5.10: A comparison of range estimation error for the set of 243 events that used the Baseline sound speed for in situ group velocity estimation. The colors indicate the source depth, darkening with depth, and the shapes indicate the multipath structure. Because this plot is square, the shaded region shows where the updated algorithm is less accurate than the in situ algorithm.

with more data, we can see that the multipath structure seems to connect different 45 degree bands across different source depths, i.e., they have the same offset from unity. These bands connecting source depth would indicate that the multipath plays a dominant role in determining the group velocity, and in turn, the range estimate. There are still smaller, parallel clusters with 
Improvement of range estimation error

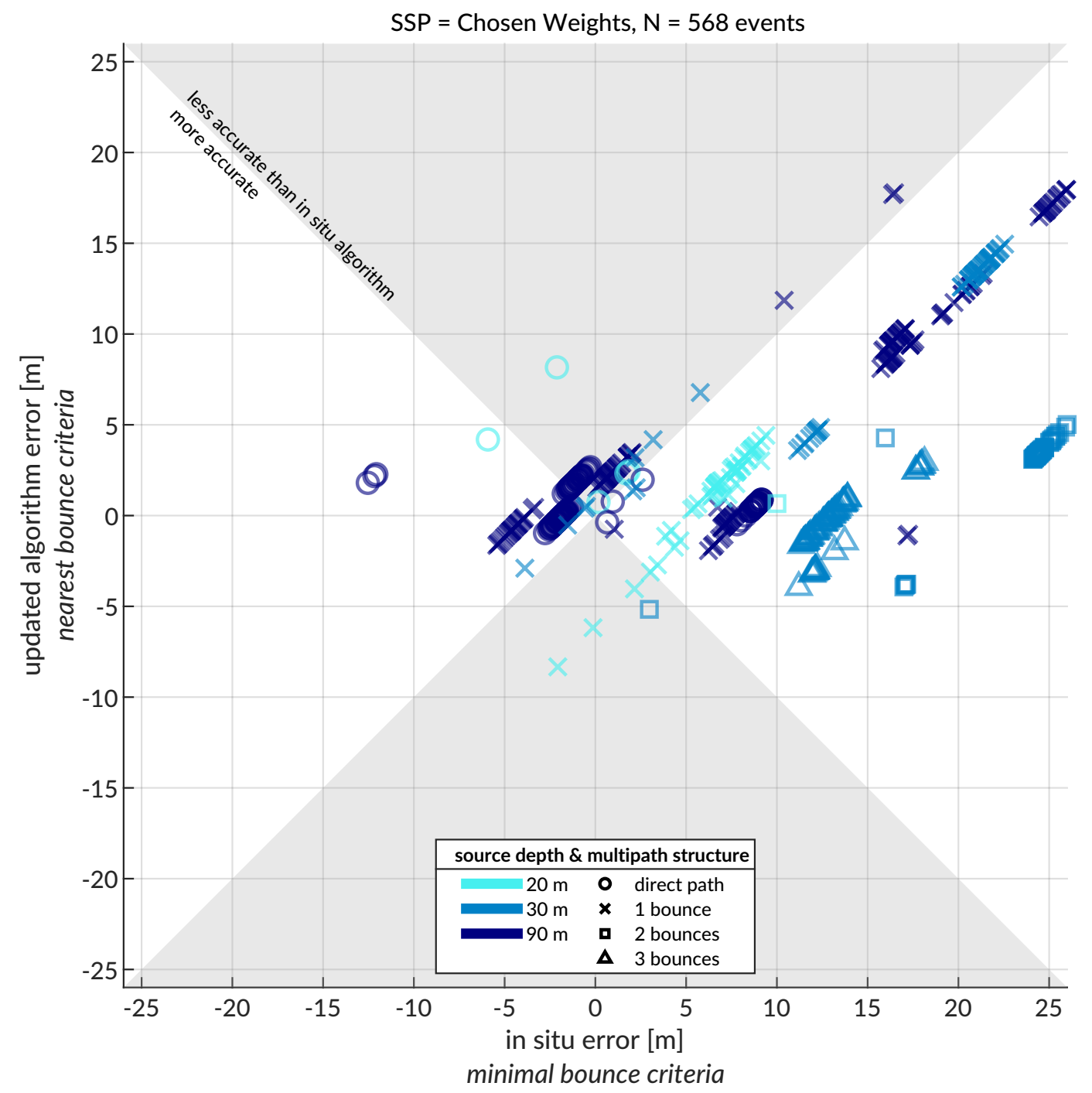

Figure 5.11: A comparison of range estimation error for the set of 568 events that used the chosen EOF weights for in situ group velocity estimation. The colors indicate the source depth, darkening with depth, and the shapes indicate the multipath structure. Because this plot is square, the shaded region shows where the updated algorithm is less accurate than the in situ algorithm.

the same source depth and multipath structure that would indicate differences in propagation ranges. One novel feature compared to the baseline SSP is the predominance of direct path events that perform better in the in situ method; this is most likely due to the plane wave approximation 
becoming more precise than the gridded. Even though the improvement is limited to 2-3 meters, this would also indicate that the weighted sound speed is the more representative SSP of the two.

The banding seen in both figures reflects GPS drift for the same node to node connection. Table 5.2 summarizes range estimation metrics as absolute error for comparison. Both the baseline and chosen weights perform an order of magnitude better with the nearest bounce criteria compared to the minimal bounce criteria, effectively exploiting multipath for ranging. The counter-intuitive result that the baseline sound speed is slightly more accurate is more likely due to the imbalance of sound speed events than it being the more representative sound speed.

\begin{tabular}{r|cc|cc}
\hline & \multicolumn{2}{|c|}{ Baseline $(\mathrm{n}=243)$} & \multicolumn{2}{c}{ Chosen Weights $(\mathrm{n}=568)$} \\
algorithm & minimal & nearest & minimal & nearest \\
\hline mean absolute error [m] & 11.38 & 2.61 & 11.36 & 4.53 \\
median absolute error [m] & 11.96 & 1.58 & 11.40 & 2.42 \\
standard deviation [m] & 4.23 & 2.70 & 8.12 & 5.05 \\
\hline efficacy [\%] & \multicolumn{2}{|c|}{99.18} & \multicolumn{2}{|c}{84.68} \\
\hline
\end{tabular}

Table 5.2: A comparison of range estimation metrics for the two sound speed inputs used during ICEX20, for the minimal bounce and nearest bounce criterion. The efficacy is the percentage of events that perform better in the updated, nearest bounce algorithm as compared to the in situ, minimal bounce algorithm. The results here replicate those from table 5.1.

\subsection{EXTENDED COMPARISON BETWEEN GROUP VELOCITY}

\section{ALGORITHMS AND SOUND SPEED ENVIRONMENTS}

Due to the operational constraint of only one modem running the vehicle behavior at a time, the previous analysis has looked at 811 total beacon to beacon events, where 243 use the baseline SSP and 568 use the weighted SSP. While overall statistics are presented, and individual events are 
compared for the same SSP between the two algorithms, the events themselves are not equally distributed with respect to depth, range, and sound speed status.

There are 1242 total beacon to beacon events in the modem experiment that would have been accepted by the ICNN for real-time ranging and navigation had the vehicle behavior been running on all modems. The value for the extrapolated range, $\hat{r}$, is only tracked when the modem runs the vehicle behavior; thus we replace $\hat{r}$ with the GPS-tracked range for all modem events. Naturally, because $\hat{r}$ converges to the correct solution, a comparison of $\hat{r}$ with the GPS-tracked range shows a normal, zero-centered distribution within the bounds of GPS drift. The analysis here therefore seeds "omniscient" knowledge of the range and leverages the post-processing pipeline to more thoroughly evaluate the acoustic range estimate for all modem events, with all three sound speed sources, and both group velocity criterion. Thus, the results in this section evaluate the utility of the algorithms and sound speed sources, divorced from their role in the ICNN. We first compare the post-processing results between the two algorithms, and then take a step-by-step approach to examine how the nearest bounce algorithm works in more detail.

\subsubsection{COMPARING BOTH GROUP VELOCITY ALGORITHMS IN POST-PROCESSING}

The previous section provided strong but limited evidence for the effectiveness of the nearest bounce criterion. This section compares the nearest and minimal bounce criteria for all events in the post-processing pipeline. The results here for the MBC are different than the in situ results due to the lack of a dynamic sampling grid and plane wave approximation. It is important to note that because the post-processing pipeline is the same for both methods, that the efficacy of the NBC compared to the $\mathrm{MBC}$ is $100 \%$, as the former simplifies to the latter for direct path classifications. 

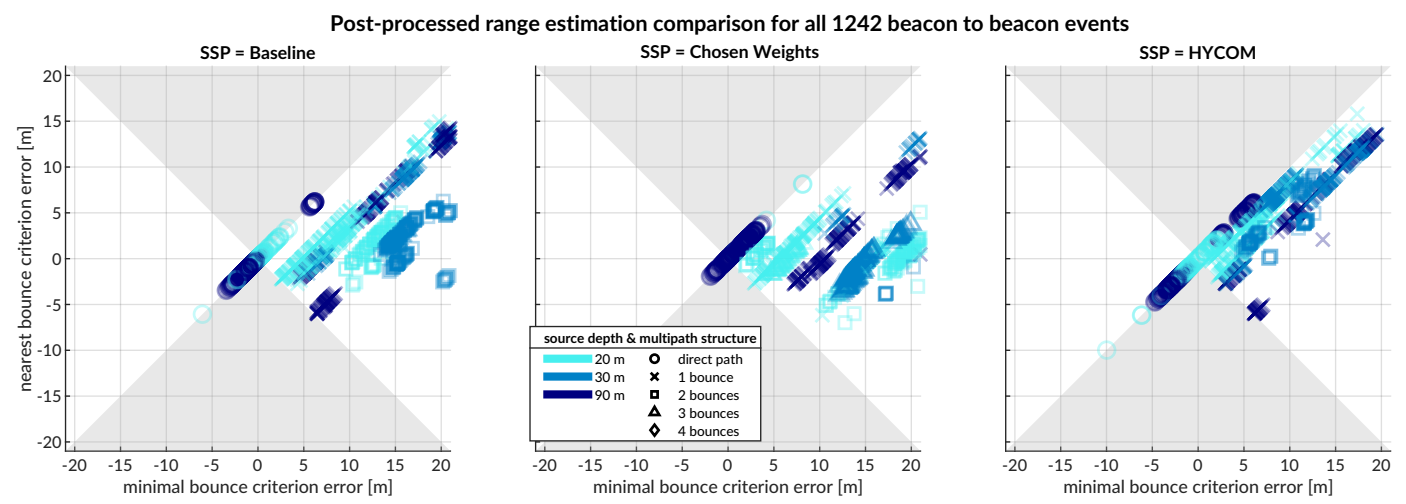

Figure 5.12: A comparison of range estimation error for all beacon to beacon events in post-processing. From left to right, the SSPs are the baseline, the chosen weights, and HYCOM. The colors indicate the source depth, darkening with depth, and the shapes indicate the multipath structure. Because this plot is square, the shaded region shows where the updated algorithm is less accurate than the in situ algorithm.

Figure 5.12 is a crossmap for all three SSPs for both group velocity predictors, with the minimal bounce on the $\mathrm{x}$-axis and the nearest bounce on the $\mathrm{y}$-axis; the important range estimation metrics are summarized in table 5.3. All plots show the same 45 degree banding indicating GPS drift. The direct path classifications lay on the line of unity as the NBC defaults to MBC. The gridded approach and multipath classification build in the computational cushion for both minimal error and minimal bias in the range estimate.

The improvement from MBC to NBC is most evident for the realistic sound speed; while the HYCOM SSP improves from a median absolute range error of 6.41 to $4.61 \mathrm{~m}$, the baseline SSP improves from 10.30 to $2.27 \mathrm{~m}$, and the weighted SSP improves from 13.28 to $2.12 \mathrm{~m}$. Table 5.3 shows further statistics on the absolute range error by SSP and group velocity algorithm. The order of magnitude improvement in the ducted SSPs demonstrate the effectiveness of the algorithm exploiting the multipath conditions. The gridded approach and multipath classification build in the computational cushion for minimal error and minimal bias compared to direct eigenray assessment. 


\begin{tabular}{r|cc|cc|cc}
\hline & \multicolumn{2}{|c|}{ Baseline } & \multicolumn{2}{c|}{ Chosen Weights } & \multicolumn{2}{c}{ HYCOM } \\
algorithm & minimal & nearest & minimal & nearest & minimal & nearest \\
\hline minimum [m] & 0.01 & 0.00 & 0.00 & 0.00 & 0.11 & 0.01 \\
$25^{\text {th }} \%[\mathrm{~m}]$ & 4.96 & 0.99 & 6.26 & 0.95 & 3.30 & 2.25 \\
median [m] & 10.30 & 2.27 & 13.28 & 2.12 & 6.41 & 4.61 \\
$75^{\text {th } \%[m] ~}$ & 15.81 & 5.51 & 19.75 & 4.11 & 10.92 & 7.46 \\
maximum [m] & 22.52 & 14.96 & 1491 & 20.21 & 19.55 & 15.81 \\
\hline
\end{tabular}

Table 5.3: A comparison of range estimation metrics for each sound speed source and group velocity estimation algorithm for all 1283 beacon to beacon events via post-processing. The $0^{\text {th }}$ (minimum), $25^{\text {th }}, 50^{\text {th }}$ (median), $75^{\text {th }}$, and $100^{\text {th }}$ (maximum) percentiles are shown to the range resolution afforded by the WHOI Micro-Modem.

\subsubsection{Further analysis of The ANOMALOUS BEACON TO BEACON CONNECTION}

As shown in table 5.3, there is a striking maximum range error of $1491 \mathrm{~m}$ for weighted SSP in the minimal bounce criteria. This section isolates the events that encompass that outlier for further examination, summarized in table 5.4. There are 10 events from South transmitting at $30 \mathrm{~m}$ depth to North receiving at $30 \mathrm{~m}$ depth. The OWTT spread is from 2.1958 to $2.1963 \mathrm{~s}$; the naive group velocity is 1429.3 to $1430.1 \mathrm{~m} / \mathrm{s}$; and the GPS-tracked range is from 3138.54 $\mathrm{m}$ to $3140.87 \mathrm{~m}$. This example ends up being an excellent case study for how sound speed and multipath fidelity work in concert to minimize range error.

The large error is driven by the MBC unexpectedly defaulting to a bottom bounce, with a much greater OWTT. Thus the NBC "correctly" classifies the multipath as 4 bounces, reducing the range error from greater than a kilometer to less than a meter. While there is no actual way of knowing if this is the correct multipath structure, the range error is remarkably small. This pattern of not choosing the minimal observed bounce structure is consistent across all SSPs; the baseline goes from 1 to 3 bounces and HYCOM goes from 0 to 2 bounces. Notably, the baseline 


\begin{tabular}{r|cc|cc|cc}
\hline & \multicolumn{2}{|c|}{ Baseline } & \multicolumn{2}{c|}{$\begin{array}{c}\text { Chosen Weights } \\
\text { minimal }\end{array}$} & \multicolumn{2}{c}{ Hearest } \\
algorithm & minimal & nearest & minimal & nearest \\
\hline \# bounces & 1 & 3 & 1 & 4 & 0 & 2 \\
mean OWTT [s] & 2.1853 & 2.1943 & 4.1812 & 2.1955 & 2.1877 & 2.1902 \\
mean group velocity [m/s] & 1436.7 & 1430.8 & 750.88 & 1430.0 & 1435.1 & 1433.4 \\
range error [m] & 15.4 & 2.39 & -1491 & 0.77 & 11.9 & 8.30 \\
\hline
\end{tabular}

Table 5.4: A comparison of range estimation metrics for each sound speed source and group velocity estimation algorithm on all 10 communication events between North at $30 \mathrm{~m} \&$ South at $30 \mathrm{~m}$.

and HYCOM range errors are never egregiously large, but are nonetheless improved with the NBC algorithm. Thus, for acoustically complex environments, the NBC has a disproportionately positive impact as the estimated SSP approaches the desired SSP.

In comparison to the earlier eigenray traces, the results here distill the importance of the gridded approach to curate multipath propagation. The grid gives BELLHOP tolerance for finding solutions that otherwise may not be found in a center or single point solution; the limitations of numerical computation, especially in a real-time environment, are more adeptly addressed by baking in a small amount of uncertainty than trying to prescribe an exact solution. This creates more data to sort through given that BELLHOP is a greedy estimator of eigenrays, but is computationally simpler than running a dense ray fan for precise eigenray finding.

\subsubsection{Post-PRoCESSED PREDICTED GROUP VELOCITY}

The nearest bounce algorithm is applied using the three sound speed inputs shown in figure 5.3; the resulting predicted group velocities are shown in figure 5.13. There are two important (and co-dependent) findings in the structure of group velocities with respect to OWTT-sound speed source and multipath structure. 


\section{Post-processed group velocity estimates by source and receiver depths}

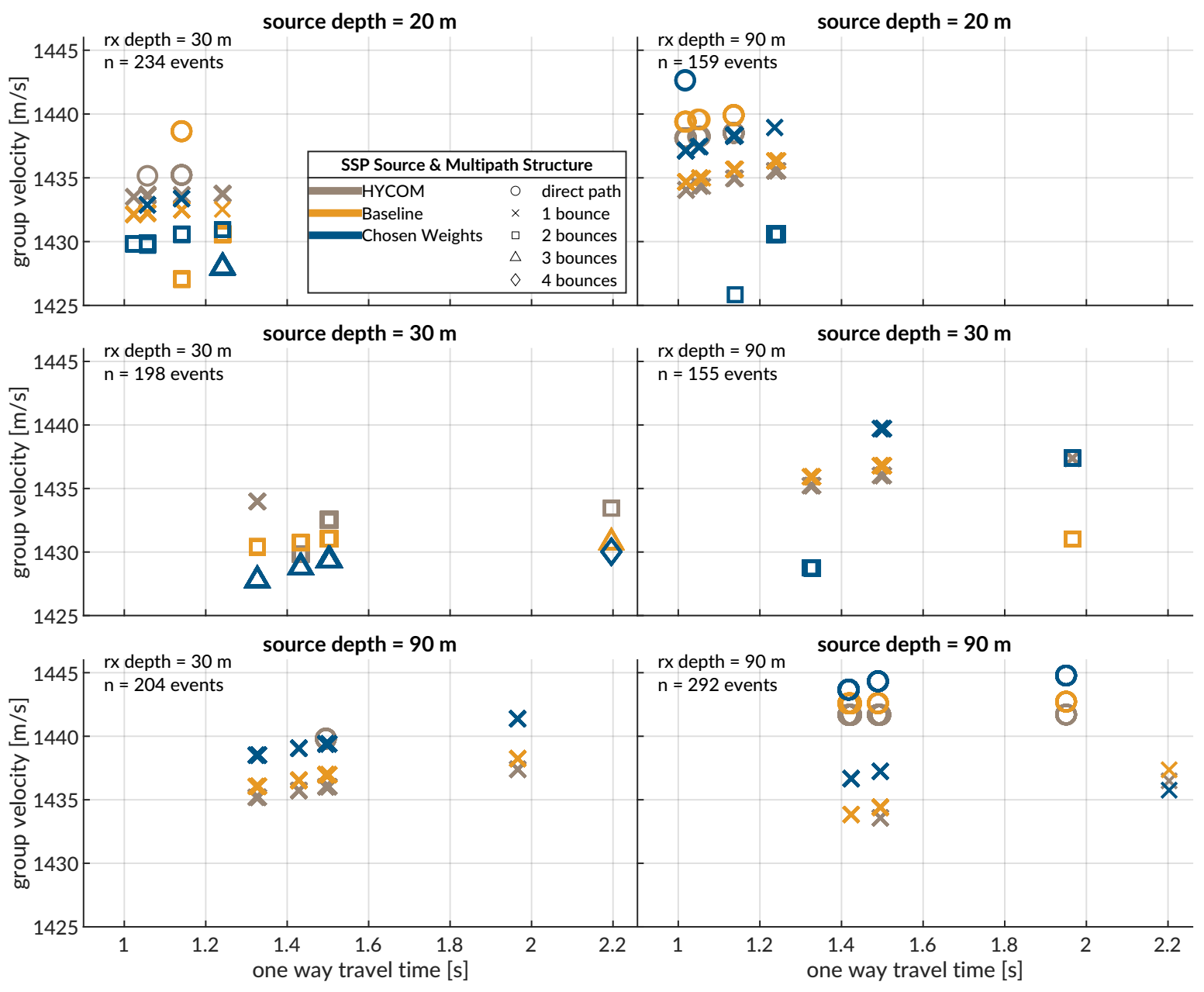

Figure 5.13: The post-processed group velocity estimates by source and receiver depth for all events. Here the marker shape refers to the number of bounces, identified by BELLHOP, and the color refers to the sound speed source. The number of events is shown underneath the receiver depth in the top left.

The sound speed inputs not only modify the predicted group velocity, as seen by colored vertical offsets, but are often encoded by different number of bounces. Intuitively, HYCOM sees the most direct and 1 bounce multipath structures, favoring faster group velocities; the weighted sound speed sees the most double and triple bounces, favoring slower group velocities; the baseline sound speed exists between the two other SSP. This is easily explained by the prominence of 
a lens forcing increased surface interactions. The gridded approach to predicting group velocity (and thereby estimating range) adds in the necessary redundancy that is otherwise not achieved by individual eigenrays.

Overall, for all sound speed sources, the group velocities are slowly varying in range and multipath condition, best demonstrated by source-receiver depth pairings between 30 and $90 \mathrm{~m}$. In some cases, small OWTT fluctuations are sorted into different multipath structures, and accordingly different group velocities, best demonstrated by the $20 \mathrm{~m}$ source depth and when both the source and receiver depth are $90 \mathrm{~m}$. This reflects the much smaller contribution from the ray traveling vertically to the source and receiver depths compared to the ray traveling horizontally between them. Most importantly, by classifying multipath structure, the spread in group velocity is more similar to the naive calculations than the in situ predictions with the minimal bounce criteria. This increased similarity invites more accurate range estimation.

\subsubsection{Post-PRocessed RANGE ESTIMATION METRICS}

The range error indicates the directional difference between the predicted range and the GPStracked range. Figure 5.14 shows the directional range error footprints for all three sound speed inputs with respect to OWTT, separated by source and receiver depth configurations. The weighted SSP range error generally has the smallest and most zero-centered footprint. The one case it does not is for the source-receiver pairings between 30 and $90 \mathrm{~m}$ in depth. The increased error for these reciprocal transmission paths is most likely driven by the computational artifacts propagating through the steep sound speed gradients of the lens, through a shadow zone. There is also the possibility that the CTD itself is slightly mis-calibrated and underestimates the depth of the lens, demonstrated by the difference in the thermocline between the RBR and XCTD. All other 
source depth pairings are significantly improved using the chosen weights compared to HYCOM or the baseline. These results, regardless of sound speed source fidelity, show an impressive improvement compared to a deterministic group velocity, where the error would grow unbounded with increasing range or OWTT.

Post-processed range error by source and receiver depths

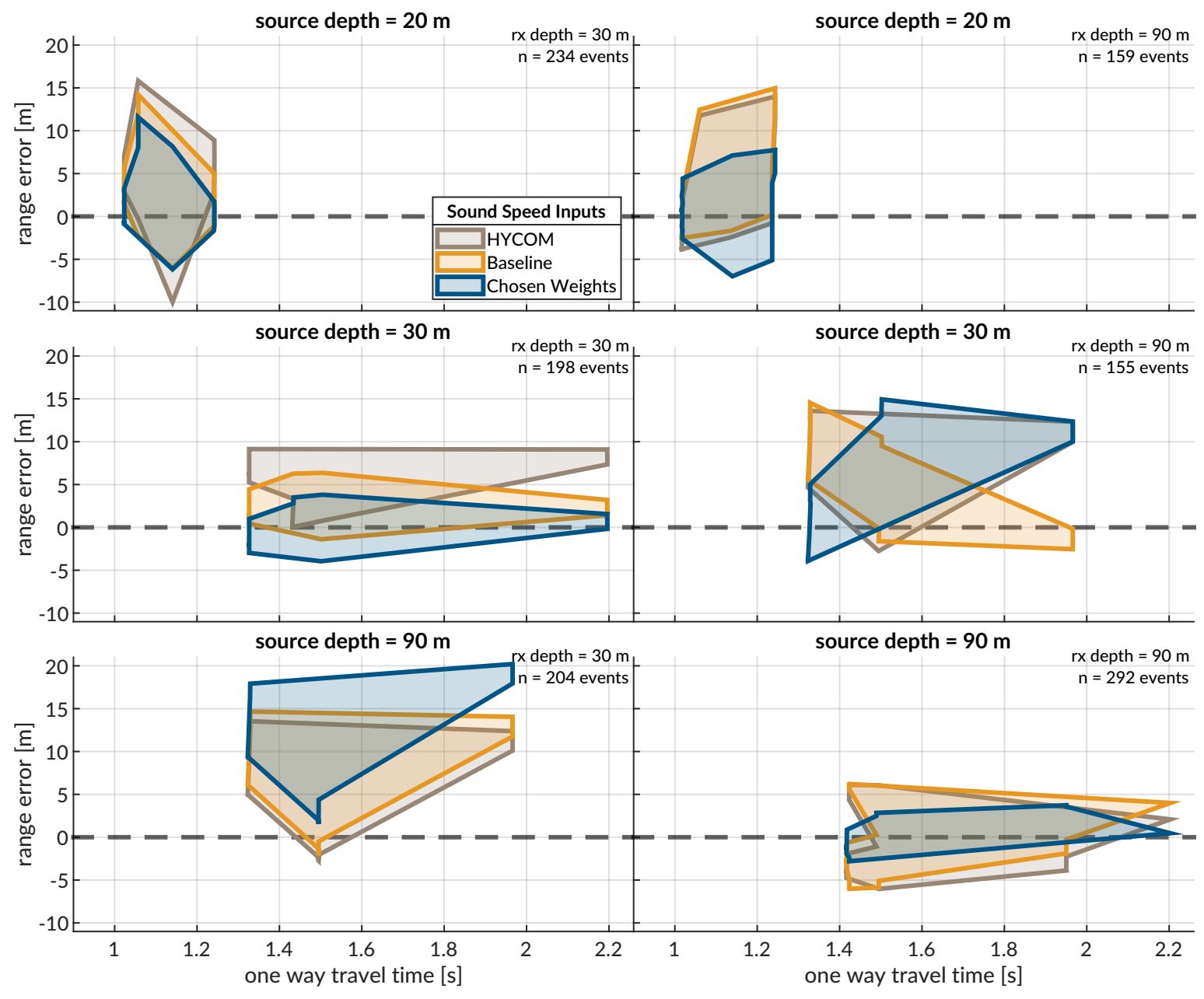

Figure 5.14: The post-processed range error for source depths of 20,30, and $90 \mathrm{~m}$, and receiver depths of 30 and $90 \mathrm{~m}$. The dashed gray line shows no error. The shaded region connects the range performance across all events. This is easily compared to the in situ range error in figure 5.9.

Figure 5.15 shows the overall range error for all events, regardless of source or receiver depth. The top part shows a histogram for range error in 2 meter bins; the bottom shows a box plot. The 
distribution of the range error for all SSPs is non-Gaussian, and appears to be bi- or tri-modal. HYCOM shows local peaks at errors between -5 to $-3 \mathrm{~m}$ and between 5 to $7 \mathrm{~m}$. The baseline SSP shows a local peak at -3 to $-1 \mathrm{~m}$ and 11 to $13 \mathrm{~m}$. The weighted SSP shows a local peak at -3 to $-1 \mathrm{~m}$, 11 to $13 \mathrm{~m}$, and also 17 to $19 \mathrm{~m}$. Even though the ducted SSPs have the same local peak close to -1 to 1 error, the weighted SSP has significantly more events that span the same magnitude of error, i.e., between -3 and $3 \mathrm{~m}$ in range error. And both ducted SSPs are sensitive to positive outliers, which seem to increase with the duct strength itself. While the weighted SSP has the narrowest spread for the $25^{\text {th }}$ to $75^{\text {th }}$ percentile of range error, it also has the highest overestimation. The overestimation is likely driven by the previously observed mismatch in reciprocal events between 30 and $90 \mathrm{~m}$ in depth. This framing, while useful for evaluating the weighted SSP as producing slightly better range estimates, obscures the impact of multipath in driving the range estimate.

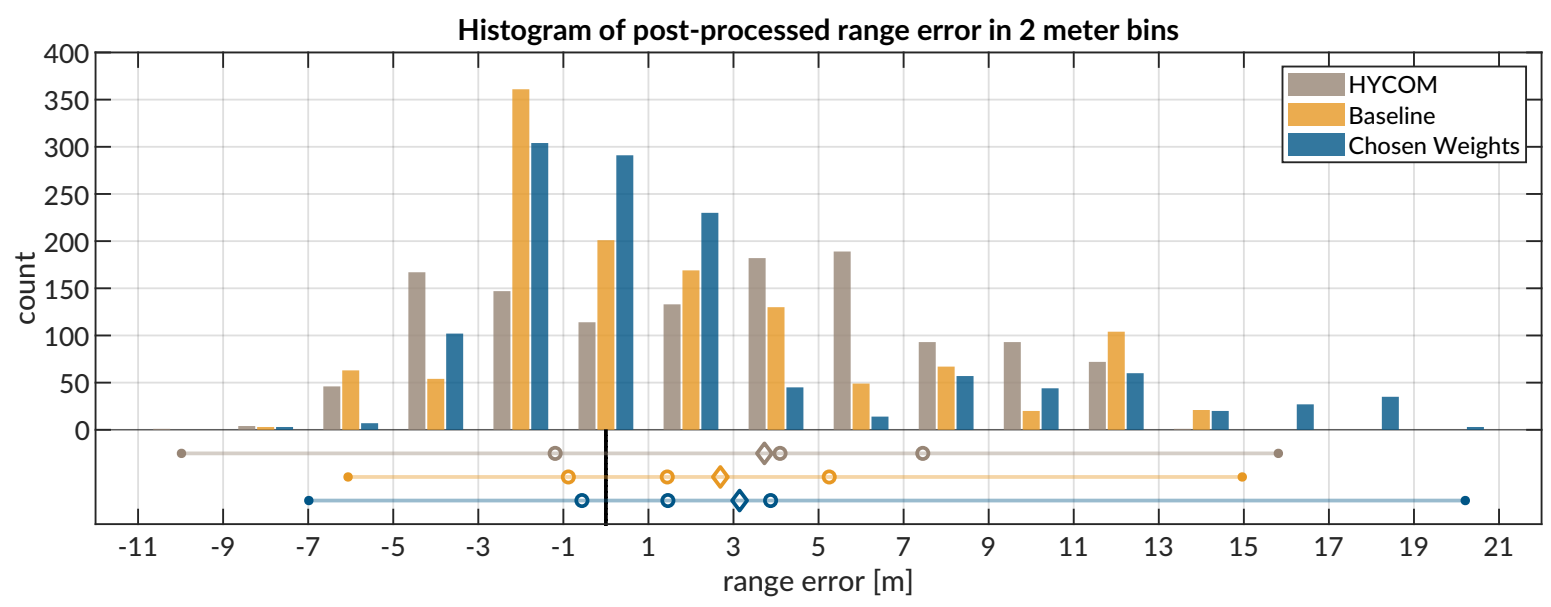

Figure 5.15: A histogram of the range error by sound speed input. Below the histogram is a minimal box plot. The closed circles on the ends indicate the minimum and maximum. The open circles indicate the $25^{\text {th }}, 50^{\text {th }}$ (median), and $75^{\text {th }}$ percentiles. The diamond indicates the mean.

Figure 5.16 demonstrates the range error in the same 2 meter bins by the number of bounces indicated in the nearest bounce criteria. The range error distributions show a coupling between sound speed input and multipath structure. Both the HYCOM and baseline SSPs are dominated 
by 0,1 , and 2 bounces; the weighted SSP classifies a significant amount of arrivals as 3 and 4 bounces as well. The increase of the number of bounces strongly matches the prominence of the duct forcing faster surface interactions. These results show that, for all SSPs, not including direct path, the range error decreases and centers around zero as multipath becomes more complex. This is evident by the weighted SSP, in particular, where the range error domain is -5 and 3 $\mathrm{m}$ for three bounces, and -3 and 1 for four bounces. Generally, multipath is viewed as an obstacle to ray path identification and range estimation; here we see strong evidence that the proposed algorithm can leverage the time of arrival to exploit multipath as additional information in reducing range error.

This framing also decouples the previously observed bi- and tri-modal distribution in range error by the number of bounces. For direct path, HYCOM and the baseline tend to underestimate; the chosen weights are fairly zero-centered. For one bounce, HYCOM favors overestimation from 5 to 7 meters; the baseline and weighted SSPs are tri-modal, with peaks at -3 to $-1 \mathrm{~m}, 7$ to $9 \mathrm{~m}$, and 11 to $13 \mathrm{~m}$. For two bounces, HYCOM is fairly uniformly distributed, as two bounces would be quite rare without the lens over smaller operating distances. The baseline SSP appears to be normally distributed around a range error of $1 \mathrm{~m}$. The weighted SSP is similarly normally distributed with a significant outlier at 9 to $11 \mathrm{~m}$. Only the weighted SSP classifies a significant amount of arrivals for three and four bounces, and they are largely zero-centered compared to the other bounce-specific distributions.

However, it is fair to say that this is an over-analysis of range error given the dominance of single digit accuracy. Considering that GPS accuracy is on the order of $5 \mathrm{~m}$, and much of the literature describes localization errors on the order of tens to hundreds of meters, the results here are remarkably accurate, regardless of sound speed input. This speaks to the dominance 
Histogram of post-processed range error by number of bounces
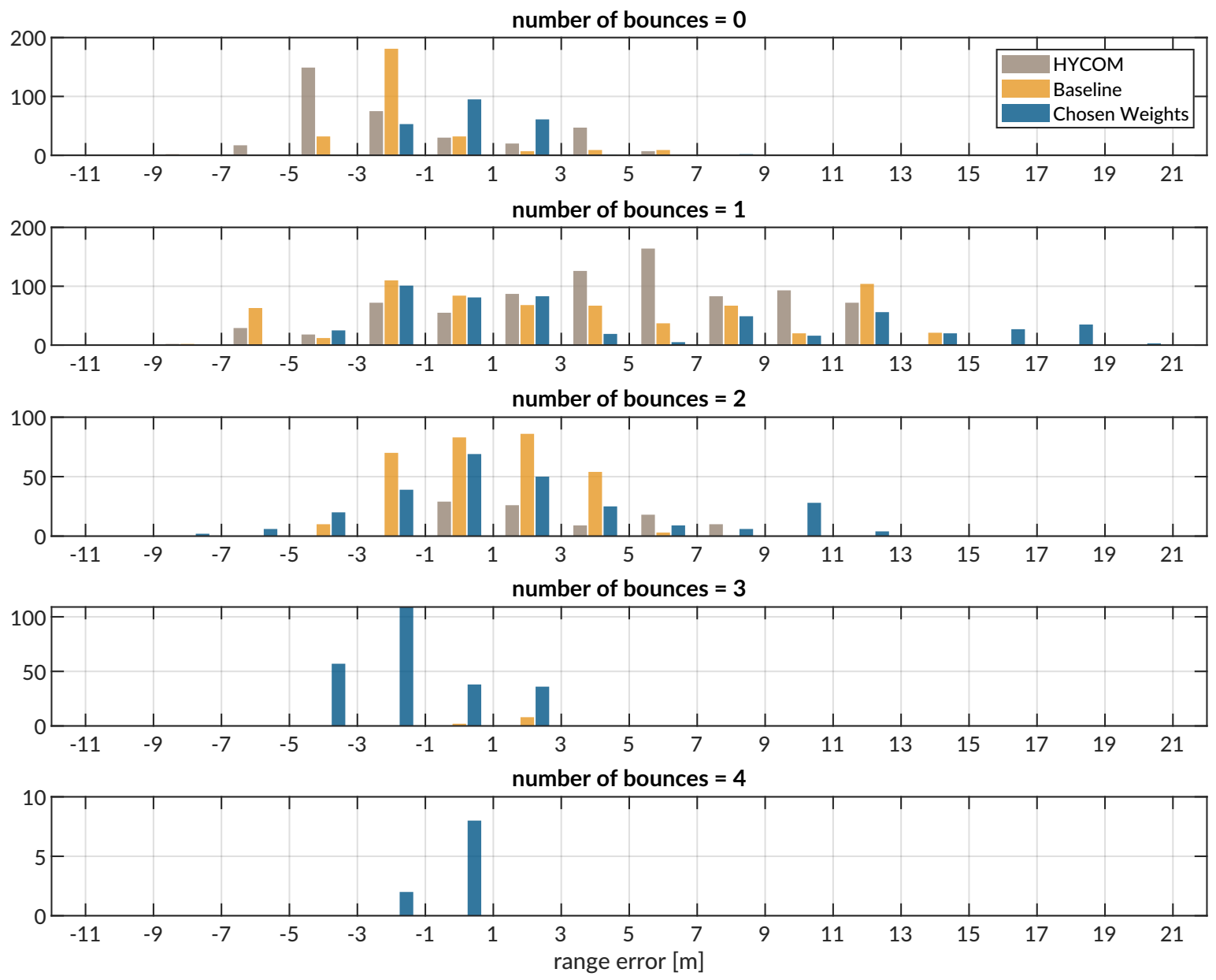

Figure 5.16: A histogram of the range error showed in figure 5.15, sorted by the number of bounces as identified in the minimal bounce criteria.

of the nearest bounce criterion more so than the sound speed fidelity in capturing a local group velocity. 


\subsection{Limitations of GPS AT POlar latitudes}

Systemic GPS drift drives the severe banding found in comparisons of range estimations via both group velocity algorithms, with those bands showing $100 \%$ positive correlation between methods and being a similar size in meters. Thus, while this thesis is primarily motivated by achieving similar accuracy and precision of GNSS, the impressive results from acoustic ranging necessitate a discussion about the limitations of GPS, particularly at polar latitudes.

The "Global Positioning System Standard Positioning Service Performance Standard", published by the Department of Defense in Apr 2020, ${ }^{10}$ claims that well-designed GPS receivers have been achieving a horizontal accuracy of 3 meters and a vertical accuracy of 5 meters, 95\% of the time. But "well-designed" is an ambiguous enough term that obfuscates two confounding factors for high latitude GPS drift-operational limitations and ionospheric scattering.

There are two metrics that demonstrate the operational limitations of GPS tracking technologies-fix rate and positional dilution of precision (PDOP). ${ }^{11}$ Fix rate looks at the ratio of successful fixes to overall attempts in acquiring a location. In animal tracking studies in polar latitudes, fix rates have significantly improved in the last twenty years, from as low as $26 \%$ in $1996^{12}$ to $87.2 \%$ in $2018 .{ }^{13}$ The latter study showed a precision of $4.3 \pm 4.0 \mathrm{~m}$ and an accuracy of $6.0 \pm$ 4.7 $\mathrm{m}$. While significant metric to evaluate GPS robustness, accuracy and precision, not the fix rate, explains the severe banding observed; fix rate bias, where various aspects of a landscape can induce bias by impeding the GPS receiver from acquiring its location, is not relevant given the static nature and small time window of ICEX20.

\footnotetext{
${ }^{10}$ https://www.gps.gov/technical/ps/2020-SPS-performance-standard.pdf

${ }^{11}$ Swanlund et al., "Gps performance in yukon's arctic coast".

${ }^{12}$ Moen et al., "Effects of Moose Movement and Habitat Use on GPS Collar Performance".

${ }^{13}$ Jung et al., "Accuracy and performance of low-feature GPS collars deployed on bison Bison bison and caribou Rangifer tarandus”.
} 
PDOP looks at the effect of satellite geometry on location precision, as satellite orbits are designed to optimize performance for human population density. A high PDOP value indicates a poor geometric configuration, such as satellites being tightly clustered above a GPS receiver, that limits the reliability of trilateration. The literature shows correlation between PDOP values and location error. ${ }^{14}$ PDOP is generally thought of as three-dimensional positioning and can be broken down into the horizontal and vertical components, HDOP and VDOP, respectively. The low elevation angles in polar areas are good for horizontal positioning but are susceptible to poorer vertical positioning. Overall this contributes to higher noise level in observations than usually seen in most global applications. The sparse and limited infrastructure in the Arctic make it ideal for satellite-based navigation, with some research proposing new satellite systems to accommodate increased interest in the region. ${ }^{15}$

However, both of these metrics are functions of the satellite constellation available to any given GPS receiver. The Arctic area is beyond the reach of many geostationary satellites, where they are low on the horizon and only visible for brief periods. There are only 24 active satellites for GPS, with 32 total in orbit. Given that their positions are tracked by stations on Earth, and their timing is updated twice a day via atomic clock, a reasonable hypothesis for bands of GPS error are different and/or updated satellite connections.

The second confounding factor is the ionosphere, the layer of the Earth's atmosphere roughly 80 to $1000 \mathrm{~km}$ above the surface that contains a high concentration of ions and free elections. In the ionosphere, electromagnetic signals are affected by the total amount of electrons encountered by the signal; the size of the signal delay is dependent on the carrier frequencies. The normal signal delay creates a GPS error of 5 to 15 meters during the daytime and 1 to 3 meters at night. ${ }^{16}$

\footnotetext{
${ }^{14}$ Swanlund et al., "Gps performance in yukon's arctic coast".

${ }^{15}$ Reid et al., "GNSS Integrity in The Arctic".

${ }^{16}$ https://www.unoosa.org/documents/pdf/psa/activities/2015/RussiaGNSS/Presentations/52.pdf
} 
The literature even references the total amount of electrons encountered by the "GPS raypath" as a means of explaining signal fluctuations. ${ }^{17}$ This variability is observed by seasonal and solar cycle variabilities, ${ }^{18}$ severe ionospheric storms, ${ }^{19}$ and auroral activity (increased electron precipitation). ${ }^{20}$ In fact, the aurora borealis was observed on multiple nights during ICEX20.

In a humbling twist, at polar latitudes, GNSS systems experience similar operational, technical, and environmental challenges that we observe in underwater acoustic communications; the underwater acoustics community generally seems to ignore these challenges when validating localization and navigation efforts.

Figure 5.17 highlights the GPS and observed OWTT drift relative to the ice movement for two pairs of modem buoy connections. The top two panels show GPS drift in both the $x$ and $y$ directions relative to the median $x$ and $y$ distance recorded between the two buoys. The bottom two panels indicate the GPS drift as $\delta R=\sqrt{\delta x^{2}+\delta y^{2}}$ and temporal drift, $\delta t$, relative to the median OWTT recorded between the two modems. Here we use the median, not the mean, to be more robust to outliers in the dataset. The dashed line is scaled by a group velocity of 1440 $\mathrm{m} / \mathrm{s}$, such that if there was no GPS drift, all events should exist on the line.

The left hand side of the plot shows the connections between the North and East buoys. The top shows consistent change in distance with respect to time, as the $\delta X$ measurement trends from 2 to -2 and the $\delta Y$ measurement trends from -1 to 1 meters. This indicates that the ice movement between the North and East buoys is non-rigid, i.e., they are actually moving relative to each other. The width of the scatter plot is a decent indicator for the precision of the GPS signal, as it

\footnotetext{
${ }^{17}$ Themens et al., "The nature of GPS differential receiver bias variability".

${ }^{18}$ Ibid.

${ }^{19}$ Mitchell et al., "GPS TEC and scintillation measurements from the polar ionosphere during the October 2003 storm".

${ }^{20} \mathrm{Gwal}$ et al., "GPS scintillation studies in the arctic region during the first winter-phase 2008 Indian Arctic Expedition"; Jin et al., "GPS scintillation effects associated with polar cap patches and substorm auroral activity".
} 
remains consistent across many colors of scattered points, which correspond to similar regions in time. The idea that these buoys are moving relative to each other is corroborated in the bottom plot, where some events exist on the dashed line indicating correlation between GPS movement and OWTT change. Some of the offsets between the vertical bands relate to different operational configurations of source and receiver depths. However, the large majority of events show vertical banding for the same nominal $\delta t$, indicating the amount of GPS drift.

This idea of GPS drift relative to the same OWTT measurements is also indicated by events between the other two buoys, South and West, on the right hand side. The GPS drift, on the top, shows a point cloud centered around the origin for events four hours and 13 hours after the experiment origin; thus these buoys are moving in a more rigid ice floe. There is minimal impact by source and receiver depth on the spread of OWTT. The GPS drift relative to the OWTT, on the bottom, shows a large amount of GPS drift for very little OWTT drift. The OWTT drift observed here is sensitive to the acoustic scattering, multipath, or environmental microstructure.

These are two subsets of the physical links, they cover all four GPS modem buoys except for Camp. The GPS at camp, as shown in figure 5.4, is the least accurate due to the human activity and infrastructure occluding the physical puck. A more comprehensive look at all physical link pairs is in Appendix A.. 

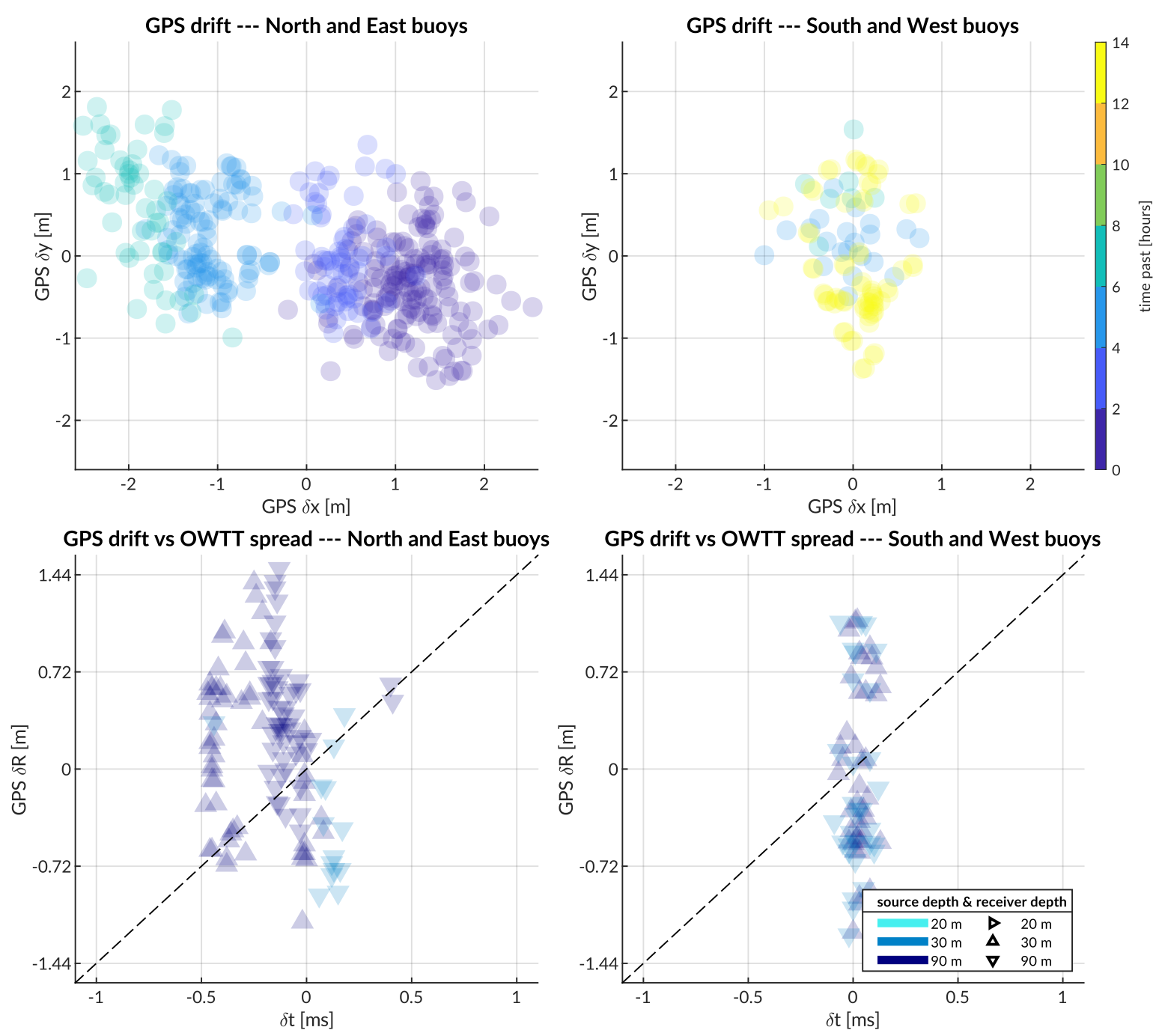

Figure 5.17: A comparison of GPS drift (top) colored by hours since the experiment origin and GPS drift against OWTT spread (bottom) demarcated by source depth and receiver depth. The physical link between North and East are shown on the left; South and West is on the right.

\subsection{Discussion}

Range estimation is an essential and often overlooked part of acoustic localization and navigation. This work solely focuses on mitigating range estimation error via a stochastic prediction of the group velocity, knowing that this can have an outsized benefit on minimizing trilateration error. We hypothesize and validate that the group velocity, especially for mission durations, is 
smoothly varying in range and multipath structure, and sensitive to source and receiver depth. We employ a GPS-tracked experiment to have a ground truth comparison for real-time localization algorithms.

This work, to our knowledge, is the first successful field deployment of a real-time, ray-based acoustic ranging system for underwater navigation. The methods here demonstrate remarkable ranging accuracy and precision for the Beaufort Lens, an acoustically complex environment. The in situ prediction via BELLHOP balances the tension in simulation fidelity versus capturing the overarching ensemble physics to approximate a local group velocity.

Furthermore, the improved nearest bounce criteria, validated in a high fidelity post-processing pipeline, produces range estimates that rival GPS accuracy and outclass GPS precision, especially at polar latitudes. It transforms multipath, widely considered as an obstacle for acoustic ranging, into a new information content to refine ranging accuracy.

The scalability of the methods provided here for long-range applications remain to be seen. Given the NBC algorithm's improvement with increased multipath, its effectiveness is likely only challenged by the valid operational scales of a range independent propagation environment. Even then, the dominant SSP amongst the variability may very well produce GPS-like accuracy. The NBC algorithm can be easily be retooled to discriminate between surface and bottom bounces, and employ simple statistical learning or more advanced machine learning techniques to select an appropriate representative group velocity. Some more advanced vehicle intelligence can toggle use the range uncertainty to toggle between a more conservative group velocity estimate and the NBC, which is intrinsically quite precise.

There are many avenues that this approach can be further refined and tested for field operations. Amongst them is defining the uncertainty grid for BELLHOP via stochastic or data-driven measures such as the distance traveled by the AUV between ICNN updates or the magnitude of 
the position corrections by the ICNN. Another is to entirely remove the seeded range and instead rely on the submerged asset's depth and recorded OWTT to find high probability fields in range, as briefly indicated in Chapter 6.

As the Arctic continues to become more accessible and navigable, safe navigation infrastructure and practices will be increasingly vital for industry, tourism, commercial, and military operations. This work contributes a new paradigm to embed environmentally adaptive behavior into AUV navigation that has demonstrated success in an acoustically complex and operationally challenging environment. Thus, we believe that this work enables more accurate range estimation, localization, and/or navigation for any field experiment given known source and receiver depths.

At the time of graduation, a majority of this chapter forms a manuscript in preparation. Bhatt, E., Víquez, O., Schmidt, H. Algorithms and experimental results for real-time, ray-based range estimation rivaling GPS accuracy and precision. Journal of Acoustical Society of America. 


\section{Preparing the Virtual Ocean for the}

\section{LAMSS SIMULATION SUITE}

This chapter focuses on the author's software contributions to the Virtual Ocean testbed, demonstrated in the context of creating benchmark cases for future work in the Irminger Sea for Task Force Ocean's “Assessing Environmental and Acoustic Realism in Tactical Decision Aids”. This is not a standalone document for how to use the LAMSS simulation suite, and should be used in coordination with the existing how-to guide in the LAMSS GitHub repository. The capabilities developed for this dissertation build on decades of work by several students, postdocs, scientists, and engineers associated with LAMSS (the Laboratory for Autonomous Marine Sensing Systems).

\subsection{INTRODUCTION TO THE LAMSS SIMULATION SUITE}

The LAMSS simulation suite is a powerful, high-fidelity, unified testbed for both manned and unmanned, passive and active acoustic systems, as well as non-acoustic sensing systems. It adds significant additional acoustic capabilities to the MOOS-IvP infrastructure (written in $\mathrm{C}++$ ). ${ }^{1}$

\footnotetext{
${ }^{1}$ Benjamin et al., "Nested autonomy for unmanned marine vehicles with MOOS-IvP".
} 


\subsubsection{LAMSS Operational Paradigm for Autonomy}

Vehicles in the LAMSS operational paradigm use the nested platform autonomy, separating the decision-making process into "frontseat" and "backseat" drivers. ${ }^{2}$ The frontseat driver is often the native software provided by the vehicle manufacturer, which deals with low level vehicle control, navigation status, battery status, sensor and actuator data, etc. The backseat driver is the culmination of several behaviors, where any single decision is distilled using an interval programming solution for multi-objective optimization. The necessary information are passed to the front seat such that all of the autonomous decision-making occurs by the backseat. Payload autonomy, by the frontseat and backseat drivers, provides fully integrated sensing, modeling, and control in a self-contained system. The system interacts with three outside connections-the frontseat (different vehicles), communication (different known and unknown acoustic signals), and sensor data (different oceanographic conditions). Significant work has gone into the design of payload autonomy interfacing such that it is agnostic to whether these are actual physical systems or simulated equivalents. ${ }^{3}$ By extension, because of the severely limited acoustic communication channel capacity, the autonomy paradigm is designed to support mission objectives without recoding or reconfiguration during a mission deployment. Instead, hierarchical mode behaviors can be toggled by a short message passed to the backseat, the centralized autonomy hub.

\footnotetext{
${ }^{2}$ Schneider et al., "Unified command and control for heterogeneous marine sensing networks".

${ }^{3}$ Schneider et al., "Model-Based Adaptive Behavior Framework for Optimal Acoustic Communication and Sensing by Marine Robots".
} 


\subsubsection{Virtual Ocean Testbed}

The Virtual Ocean testbed replicates the physical ocean environment for the frontseat, acoustic communication, and sensor data. ${ }^{4}$ It is fully linked to ocean prediction systems like $\mathrm{HYCOM}^{5}$ and MSEAS, ${ }^{6}$ and legacy acoustic models like BELLHOP, ${ }^{7} \mathrm{KRAKEN}^{8}$ and OASES ${ }^{9}$ to provide highly modular and configurable physics-based virtual experiments. Each platform operated in the simulation suite includes a modular Virtual Ocean at the necessary scale and resolution, driven by the EOF parameterization, which can serve as the input to an embedded acoustic model (BELLHOP). The parameterization also facilitates frequent environmental updates from the topside, prioritized by joint environmental and acoustic significance, transmitted via the acoustic channel. Virtual experiments with these interlocking simulation capabilities enable the development and refinement of autonomous behaviors and sensing paradigms to best exploit the acoustic environment for tactical gain. Appendix C. demonstrates how to run a generic Arctic mission with the EOF capability in more detail.

\subsection{Task Force Ocean \& The Irminger Sea}

Task Force Ocean (TFO) was established by the U.S. Navy in March 2017 to advance Navyrelevant ocean science to encourage new sensing, modeling, and prediction techniques to enhance scientific understanding of complex, coupled environments. The relevant TFO effort has

\footnotetext{
${ }^{4}$ Schneider et al., "NETSIM: A Realtime Virtual Ocean Hardware-in-the-loop Acoustic Modem Network Simulator".

${ }^{5}$ Chassignet et al., "The HYCOM (HYbrid Coordinate Ocean Model) data assimilative system".

${ }^{6} \mathrm{Haley}$ et al., "Multiscale two-way embedding schemes for free-surface primitive equations in the "Multidisciplinary Simulation, Estimation and Assimilation System"'”.

${ }^{7}$ Porter, The BELLHOP Manual and User's Guide: Preliminary Draft.

${ }^{8}$ Porter, The KRAKEN Normal Mode Program.

${ }^{9}$ Schmidt, OASES Version 3.1 User guide and reference manual.
} 
highlighted the Irminger Sea, Bay of Bengal, and the Arabian Sea; the analysis in this chapter solely focuses on the Irminger Sea, particularly a region of interest we call "Box 3 " from 40 to $50 \mathrm{~N}, 30$ to $40 \mathrm{~W}$, which is situated between the Gulf Stream and the North Atlantic Current. In relation to other work in this thesis on the Beaufort Lens, initial sound speed profiles collected in the Irminger Sea Box 3 exhibit various ducted acoustic conditions.

Given a priori investigation on significant structures and variability in the Irminger Sea through both data mining and HYCOM reanalysis, the significant and interacting capabilities of the Virtual Ocean and the nested autonomy paradigm in LAMSS/MOOS-IvP enable narrative explanations for their impact on Naval operations. Furthermore, by toggling different schema of the Virtual Ocean between current Navy models, climatologies, and data sampling behaviors, we can examine the current capabilities of Navy datastreams in virtual experiments at high-fidelity and no risk to a crewed team. Lessons learned from these virtual experiments can examine how training and onboard Tactical Decision Aids (TDAs) demonstrate recognition of those features, if at all, and how they enable operates to make use of their presence. By focusing on the Irminger Sea, an area with high vertical and temporal resolution of data extending back close to 20 years, we can quantify the interannual, decadal, and seasonal variability, as well as its impact on operational awareness.

Thus the Virtual Ocean plays an important role in teasing out a "through-the-lens" impact on acoustic operations by oceanographic structures. More precisely, it can assess which features and structures are significant, and to what scale. Tools to synthesize a 3D time-varying oceanic field, and to filter out or add in specific environmental features, allow fine-tuned control over the experiment space for vehicle behavior and acoustic propagation studies. Broadly, this effort seeks to determine what a more realistic representation of the coupled environmental and acoustic fields can look like, if the environmental representation of the sound speed profile can emphasize 
its acoustic significance, and if the uncertainty onboard a vehicle (or a submarine) can be reduced in real-time. Benchmark cases seek to find statistically representative (as opposed to rare or anomalous events) that have significant acoustical, tactical, or operational repercussions.

\subsection{Software Handshaking With Environmental Inputs}

This section will cover the current capabilities of the tuned EOF sets by briefly explaining the relevant function calls. The minimal software architecture enables the integration of any previously studied space-time region, agnostic to software preferences (e.g. MATLAB or Python). The CLamssNetCDF is a C++ class with custom functions to integrate a variety of environmental sources into MOOS process, uSVP_OceanModel. It currently supports HYCOM, MSEAS, GDEM, and the EOF files. The file format for the EOF file is a NetCDF, which takes advantage of the gridded nature and object-based structure. Here we detail the specific functions that read the EOF file:

- open_eof_nc(string nc_file) -opens and initializes the file

- get_eof_varnames () -stores variable names

- get_eof_dimension() - gets the dimensions for each of the variable names

- get_eof_depths () - gets the depth array for EOF and baseline SSP

- get_eof_weights () -gets the initial weights for a given region

- get_eof_baseval() - gets the baseline sound speed profile

- get_eof_profile(lat,lon,depth) -returns the weighted EOF sound speed for a latitude, longitude, and depth vector

Sending a message to update weights during a mission involves a web-based GUI to enter and confirm any message, with an acknowledgment receipt provided. The parameters to the mes- 
sage are driven by a compressed source encoding ${ }^{10}$ to include a timestamp, source and receiver identification, and the new weight indexes and values. The specific functions to digest an update for the EOF file during a mission:

- handle_eof_weight_update_message (index, weight_update) -modifies the weight(s) driving the EEOF sound speed

- internal_wave_eof_weight(index, x,y,t,period, wavelength, heading, amplitude_factor) -modifies the grid of weights driving the EOF sound speed to simulate features like an internal wave

As a note to the curious reader, many instances in the codebase for "EOF" are "EEOF", as the former is sometimes misconstrued as "end-of-file" and breaks compilation. The EEOF nomenclature loosely stands for Environmental EOFs, but is also a tongue in cheek reference to the spelling of my name.

\subsection{Integrating NOAA BAthymetric DATA}

The National Oceanic Atmospheric Administration (NOAA) National Center for Environmental Information (NCEI) database includes a global bathymetric product at one minute resolution. ${ }^{11}$ It is easily interfaced at the web client at: https://maps.ngdc.noaa.gov/viewers/wcs-client/ The Virtual Ocean uses a NetCDF file format for bathymetry that was previously available through the NDEI portal; as a GeoTIFF is the only available output, a small script creates the backwards compatible version. The bathymetry, shown for the Irminger Sea in figure 6.1, is used to truncate the EOF SSP to the appropriate bottom depth and provide the range dependent bottom profile

\footnotetext{
${ }^{10}$ Schneider et al., "A State Observation Technique for Highly Compressed Source Coding of Autonomous Underwater Vehicle Position", "The Dynamic Compact Control Language".

${ }^{11}$ roughly $1.85 \mathrm{~km}$ at the equator, $1.71 \mathrm{~km} \mathrm{E} / \mathrm{W}$ at $23^{\circ} \mathrm{N} / \mathrm{S}, 1.31 \mathrm{~km} \mathrm{E} / \mathrm{W}$ at $45^{\circ} \mathrm{N} / \mathrm{S}$, and $0.72 \mathrm{~km} \mathrm{E} / \mathrm{W}$ at $67^{\circ} \mathrm{N} / \mathrm{S}$
} 
for BELLHOP when necessary. This alleviates the burden of carrying bottom depth for the EOF sound speed basis.

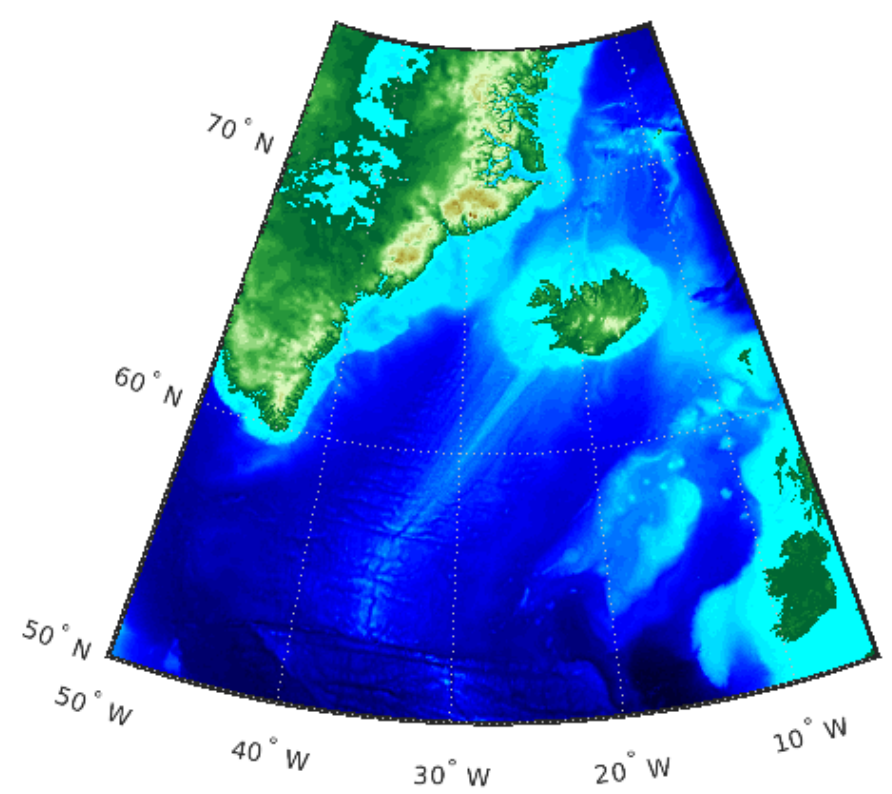

Figure 6.1: A bathymetric map of the Irminger Sea sourced from NOAA data.

\subsection{Creating Environmental EOFs}

In order to not doom virtual experiments to success, ${ }^{12}$ one can integrate a full HYCOM or MSEAS model as the "actual" ocean while submerged assets run an EOF-driven Virtual Ocean. Thus the model provides spatio-temporal dynamics while any platform can run the relevant range independent acoustic processing. Integrating the model is as simple as changing the pointer of a symbolic link before starting a mission. We use symbolic links as it is not sustainable to commit large ocean models to version control systems (but the EOF output file is on the order

\footnotetext{
${ }^{12}$ Randeni et al., "Construction of a high-resolution under-ice AUV navigation framework using a multidisciplinary virtual environment".
} 
of kilobytes). This has the additional advantage of simplifying startup scripts and behavior plugs or protobufs. In this system, the EOF file is not a prescriptive statistical measure for any region. It serves as a first order guess and can be easily switched to explore other environmental and acoustic features. This section focuses on the data assimilation, interactive visualization, and EOF confirmation pipeline for selected data in the Irminger Sea.

\subsubsection{Data Assimilation}

The first step is to curate a training set of data. The data shown in figure 6.2 was mined across several years and sorted by month by collaborators at the Naval Research Laboratory. There are a total of 2318 quality controlled profiles. The depth-dependent mean and standard deviation SSP are superimposed onto the batch. The months of March and September were chosen for their different oceanographic conditions resulting in different ducted acoustic propagation conditions. In particular, March shows well mixed surface water down to $300 \mathrm{~m}$ whereas September shows residual surface warming from summer months creating a surface duct. The SSPs are largely the same below $1 \mathrm{~km}$. In many cases, the mean profile is not representative of the actual data, artificially splicing what appears to be a more bimodal distribution; the standard deviation better matches the bimodal nature but is not necessarily a robust method to isolate representative cases.

The data assimilation scheme prioritizes data in the upper water column. By merging all data to the same depth grid, impartial data is counted as $\mathrm{NaN}$, and does not contribute to the variability at missing value depths. A corollary of this technique is that the output SSP always exists for the full depth domain observed. Data should be curated such that there is enough data at each depth grid to trust the variability amassed and encoded into the EOFs. 
For larger data sets, i.e. tens of thousands of profiles, the EOF pipeline creates a training, test, and validation datasets of $70 \%, 15 \%$, and $15 \%$ respectively. For smaller datasets, the user has the option of training on all the data or combining the test and validation datasets. For this exercise, the EOF set is trained off all profiles, regardless of season or year. This is due to the partial nature of the data, which do not extend fully throughout the water column.

\section{Sound Speeds from public data for Irminger Box 3} from NRL Stennis
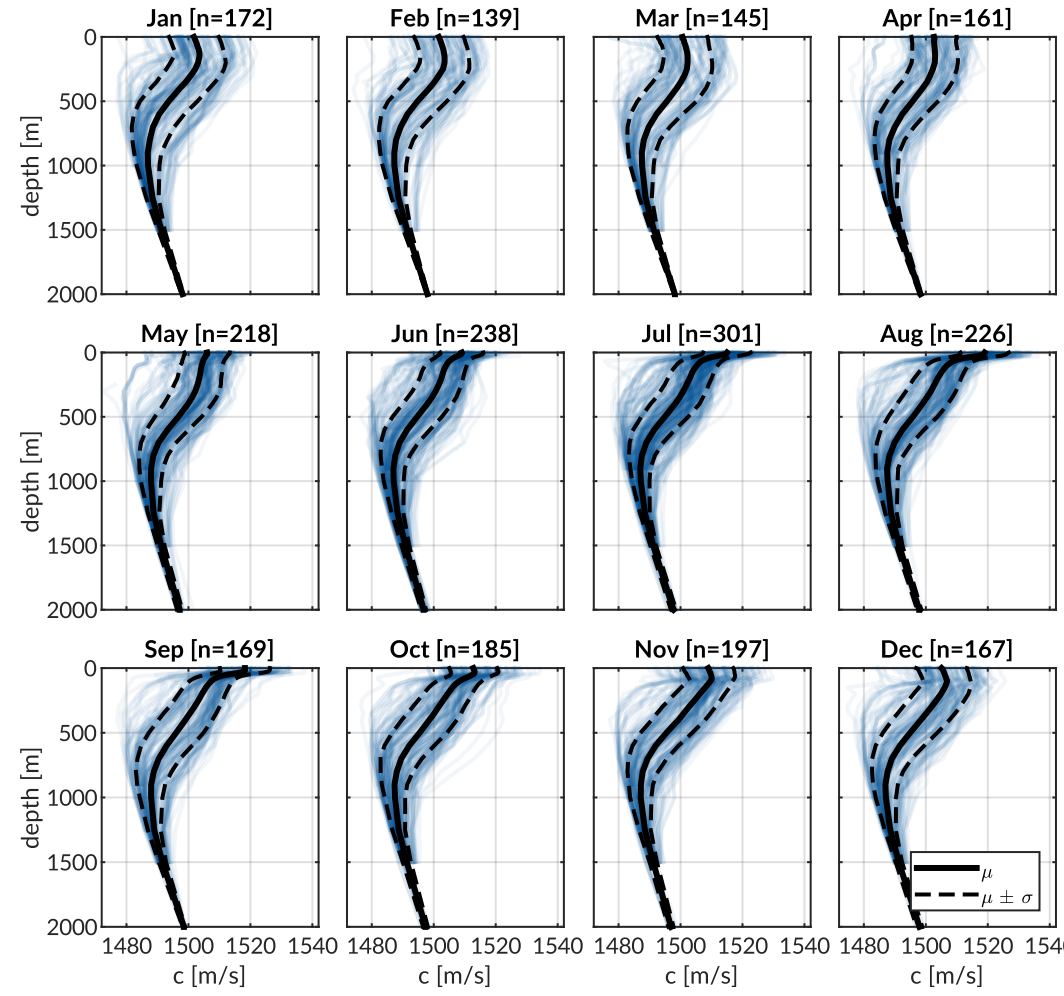

Nov [n=197]

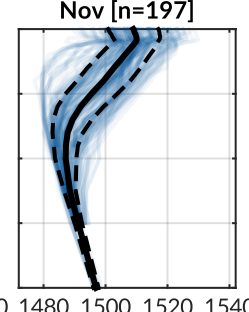

$\operatorname{Dec}[\mathrm{n}=167]$

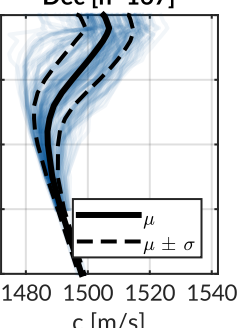

Figure 6.2: Sound speed profiles by month for Irminger Box 3. The profiles are drawn with a slight transparency to weight their information density; the mean and standard deviation are superimposed. 


\subsubsection{SMOоTHING}

EOF analysis produces functions that are optimally orthogonal to each other in describing the variability observed in a de-meaned dataset. One unfortunate consequence of this is the potentially for sharp gradients or discontinuities that may result in unrealistic sound speed profiles, which would hamper acoustic propagation models. Thus, EOF production pipeline allows the user to see how different smoothing methods alter the shape of EOFs by presenting each EOF with all smoothing methods at once. Generally speaking, the loess and sgolay methods are most robust in preserving the EOF shape while removing noise from the CTD data.

Parameterized smoothing methods often require a normalized smoothing factor to dictate the bandwidth of the smoothing window. Figure 6.3 shows how the mean absolute error (MAE) in recreating sound speeds from the SSP training set are impacted by the smoothing factor, $\pi_{s f}$, for the aforementioned smoothing methods and the number of EOF functions. The MAE decreases with increasing number of EOFs, but the relative reduction minimizes after 8 EOFs. The MAE increases almost exponentially with an increasing smoothing factor, where factors greater than 0.6 tend to severely distort the EOF shapes themselves, preventing effective SSP recreation. The visualization shown here informs a user on both how many EOFs to use and what smoothing factor to apply. In this case, I chose 8 EOFs with a smoothing factor of 0.2. While there is no hard and fast rule for either of these parameters, there is a trade-off between the MAE, the smoothing factor, and the number of EOFs required. 


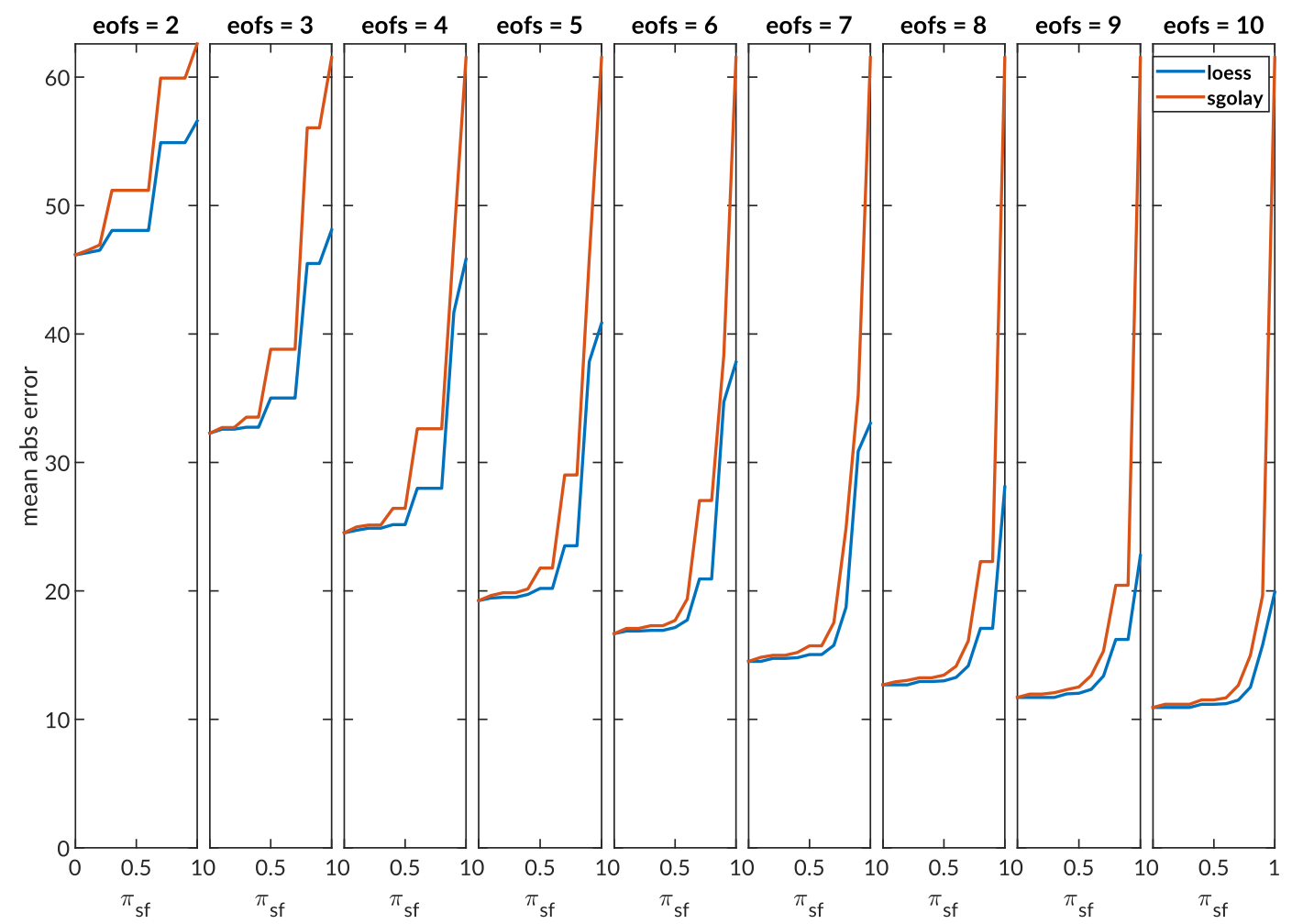

Figure 6.3: Automated visualization showing the impact of smoothing on EOFs in recreating the test set of sound speed perturbations.

\subsubsection{EOF Shapes and Weight Distributions}

There are two tangible products encoded into the EOF NetCDF file-the EOF functions themselves and a kernel density estimate (KDE) of their projected weights for the testing data.

Figure 6.4 shows the EOF shapes on the top and the variance explained on the bottom. The EOF shapes on the top panel indicate the dimensions of variability observed in the SSPs. The lower order EOFs, i.e. 1-4, are generally smoother and without many zero crossings. The higher order EOFs are generally less intuitive and flip sign quite often. Many interpret the EOF shapes themselves to be governing physical structures; this is at best taken with a grain of salt. Because the depth-dependent mean is removed in making the EOFs, these physical structures are already distorted with respect to a temperature and salinity profiles. Furthermore, they are constrained 

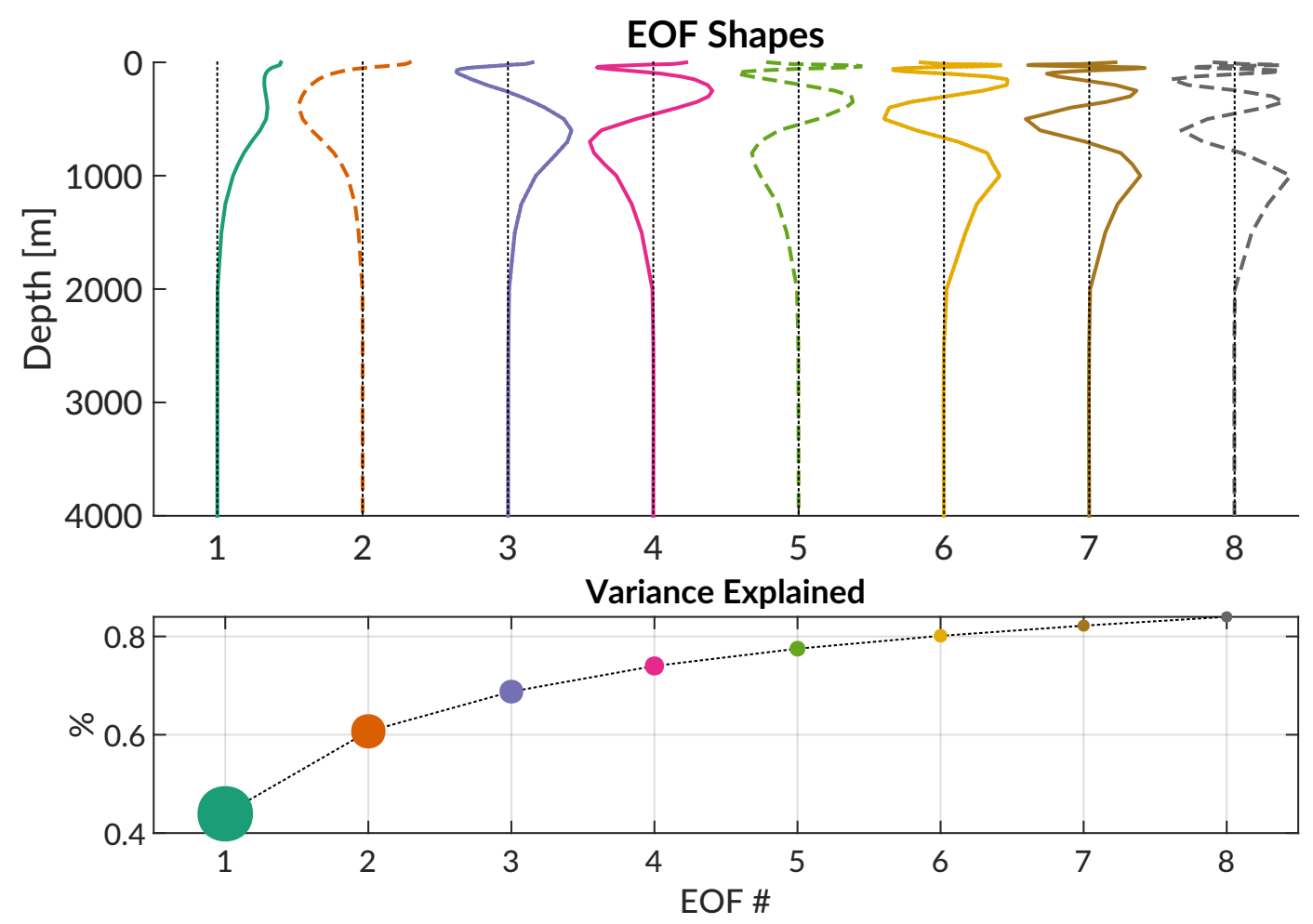

Figure 6.4: Resulting EOF shapes and their significance

to be optimally orthogonal (and then smoothed to be slightly sub-optimal), so they do not reflect in anyway physically driven mechanisms. The EOFs themselves exhibit no variability after 2000 $\mathrm{m}$. This is driven by both the lack of data at depth, simplifying the variability domain, in concert with the relatively static nature of sound speed in the deep ocean compared to that of the surface. Accordingly, the bottom panel gives further insight into the utility of higher order EOFs, and if it would be appropriate to remove them based on the additional gain in cumulative variance explained. The individual variance explained is also visually indicated by the size of the dot on the scatter plot.

Figure 6.5 shows a KDE for the weights driving SSP recreation for the test dataset. The KDE is a necessary approach to fitting a distribution to the data without imposing prior knowledge or statistical fit. It is important to note that the KDEs are inherently independent from each 

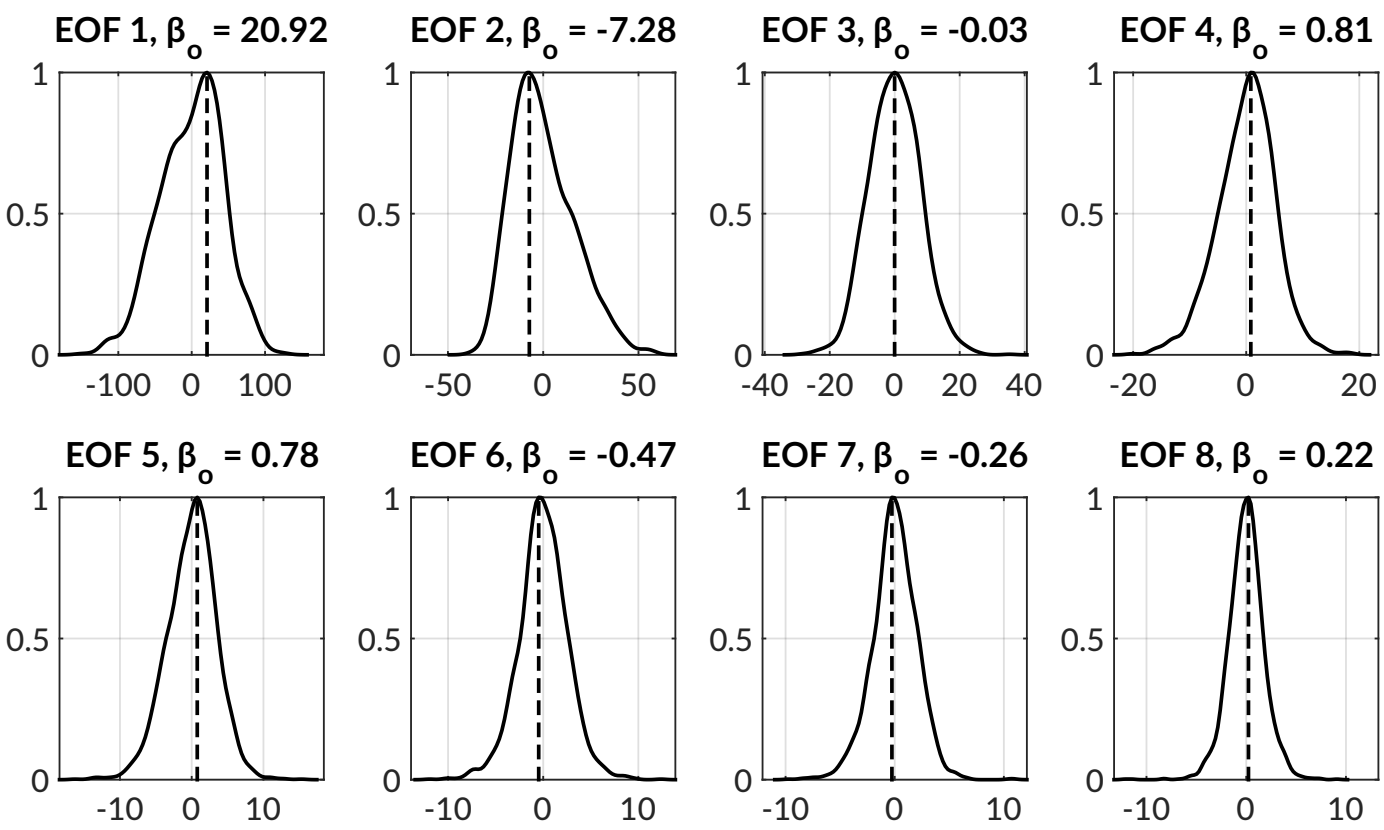

Figure 6.5: Kernel density estimates of the projected weights of the test set. The dashed line indicates the maximum likelihood of each probability distribution and that value is indicated in the title for each subplot.

other, following the orthogonality of the EOFs themselves. In an ideal dataset, these distributions would be normally-distributed and zero-centered; the amplitude bounds would decrease with higher order EOFs. This mostly holds true in data collected for Irminger Box 3; the first two leading order EOFs have a maximum likelihood estimate (MLE) at 20.92 and -7.28; the higher order MLEs exist between -1 and 1. Effectively, a secondary default SSP profile is given by these MLE weights. The distributions themselves also appear to be fairly normally distributed, unlike the ones for ICEX20 which were bi-modal. The KDE is a necessary step in the process in constraining the bounds of the message, and by extension, its byte size, for updating weights via acoustic communication. The KDE is also a welcome information set in creating internal waves, in deciding the amplitude and period for any given weight. EOFs trained on models tend to un- 
derestimate the bounds and oversimplify the KDE; EOFs trained on data tend to overestimate the bounds and introduce non-normal distributions.

\subsection{MANUALLY IDENTIFYING BENCHMARK CASES}

Identifying benchmark cases, and mapping them onto the EOF system to integrate into the LAMSS/MOOS-IvP Virtual Ocean, is a crucial element to quantifying the impact of environmental realism on acoustic operations. The first approach to finding benchmark cases is manually identifying individual CTDs that show interesting acoustic features and examining their model counterparts. This is of course extremely time intensive and not necessarily even optimal.

Figure 6.6 shows the first benchmark studies, all in September of 2007. Across all benchmark studies, the EOF system captures the HYCOM SSPs quite well. It also captures the CTD SSPs with some microstructure removal. The tension between resolving every microstructure versus understanding how pervasive that microstructure is, is only solved by omniscient data sampling. The current resolution and variability in the EOF system mirrors the available data.

The benchmarks were chosen for their differences in resolving ducts that would undoubtedly have an effect for both acoustic communication and sensing. Benchmark 1 shows significant ducting captured by the CTD but not predicted by HYCOM. Benchmark 2 shows a small difference in the minimum of the acoustic channel, but the depth of that difference is slightly below the target depths for AUV operations. Benchmark 3 shows a near opposite of benchmark 1, except a local maximum observed in the CTD actually creates a smaller duct within the broader duct. 

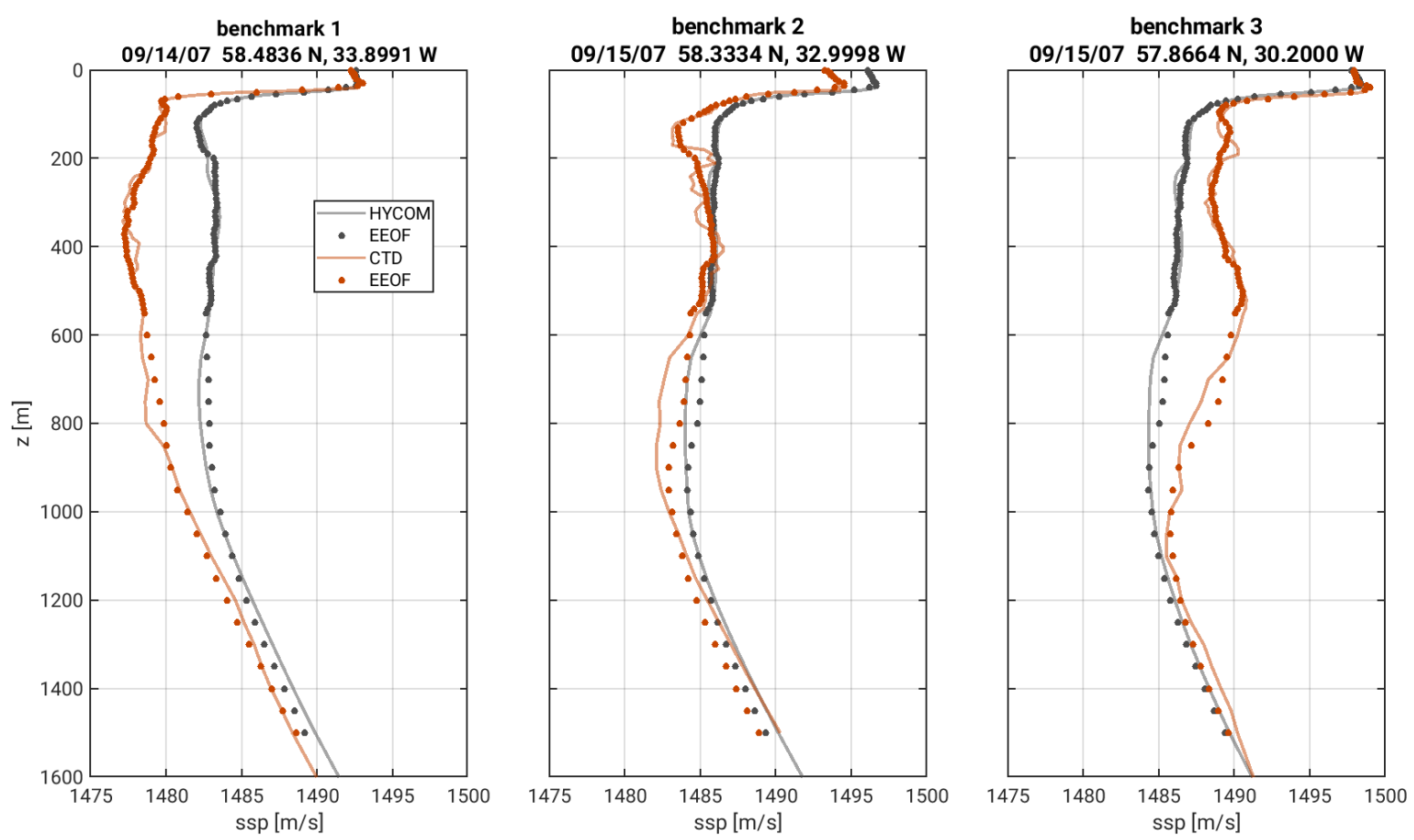

Figure 6.6: Manually chosen benchmark cases, with the CTD and EEOF estimate.

\subsection{K-MEANS CLUSTERING BENCHMARK CASES}

This section explores k-means clustering ${ }^{13}$ for each month to isolate synthetic but representative canonical profiles as benchmark cases. The data to be clustered are truncated SSPs above $750 \mathrm{~m}$ to emphasize the upper water column variability with the squared Euclidean distance metric and the kmeans++ algorithm to find centroid seeds. We choose $k=2$, informed by the bi-modal nature of the sound speed data as well the tactical knowledge that Irminger Box 3 lays on the boundary between the Gulf Stream extension and the North Atlantic Current. Figure 6.7 demonstrates two benchmark SSPs for further study from each month. The benchmark profiles are indicated by a black line with their EOF fit indicated by black circles. The clustered SSPs are indicated in blue and orange, respectively; unclustered SSPs indicate profiles that were

\footnotetext{
${ }^{13}$ Kanungo et al., "An efficient k-means clustering algorithm".
} 
not accepted by either cluster. A simple k-means clustering captures the dominant sound speed modalities of the data and is extremely well represented by the EOF system; this clustering can be improved by adding important acoustic paramters ${ }^{14}$ to the feature space. The weights to create these benchmark cases are shown in table 6.1.
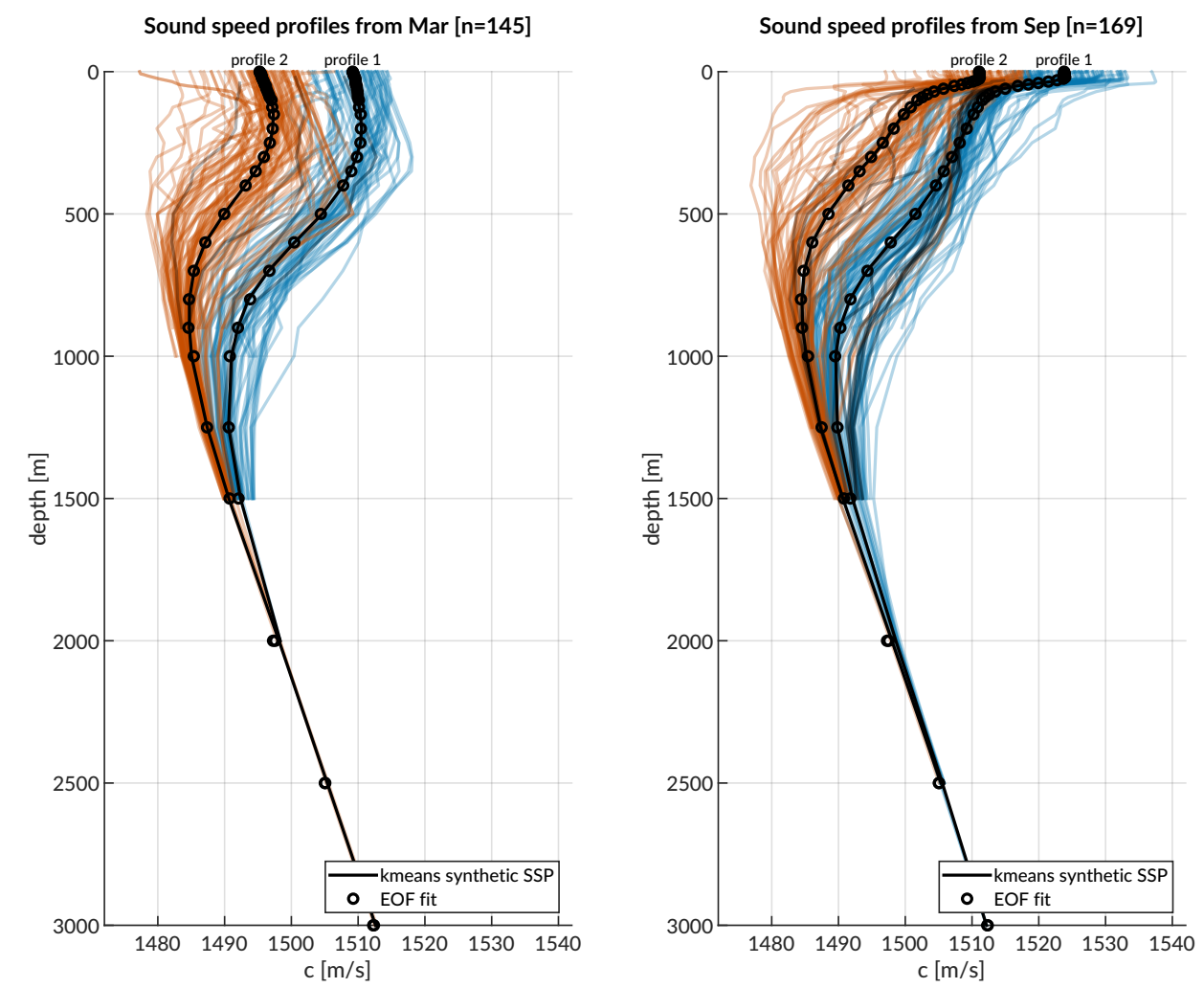

Figure 6.7: A k-means cluster $(\mathrm{k}=2)$ to identify two benchmark cases for each month.

Figure 6.8 projects the spatial boundaries of the clusters. Without any prior knowledge, the clusters are well defined into northern and southern factions, which mirrors the Gulf Stream extension and North Atlantic Current. The clustering approach separates data into an intuitive representation but also begins to indicate at what scales can we consider a volume of the ocean to be well-trained by previous data. Further data assimilation and clustering can create sensitivity

${ }^{14}$ Helber et al., "The Acoustic Parameter Climatology". 
and correlation maps for what spatial regions are well explained by current data and what regions should be a priority for data sampling.

\begin{tabular}{c|cccccccc}
\hline Benchmark & $w_{1}$ & $w_{2}$ & $w_{3}$ & $w_{4}$ & $w_{5}$ & $w_{6}$ & $w_{7}$ & $w_{8}$ \\
\hline Mar Profile 1 & -56.70 & -11.08 & 2.87 & 0.76 & 1.04 & 0.02 & -0.22 & 0.11 \\
Mar Profile 2 & 18.64 & -20.28 & 4.18 & -1.46 & -0.39 & 0.48 & 0.52 & 0.43 \\
\hline Sep Profile 1 & -4.52 & 20.33 & -3.11 & -2.04 & 2.16 & 0.47 & 0.05 & -0.43 \\
Sep Profile 2 & 58.66 & 14.70 & 2.74 & -1.30 & 2.40 & 1.59 & -0.89 & -0.49 \\
\hline
\end{tabular}

Table 6.1: EOF weights for clustered benchmark cases for the months of March and September.

The black dots indicate where the anomalous profiles exist in space. They belong to 2012, 2013 (8), 2014, 2015, and 2018, indicating that 2013 may have been an extremely anomalous year for the region. In this way, clustering can be applied for real-time verification of sensor fidelity and/or historical conditions.
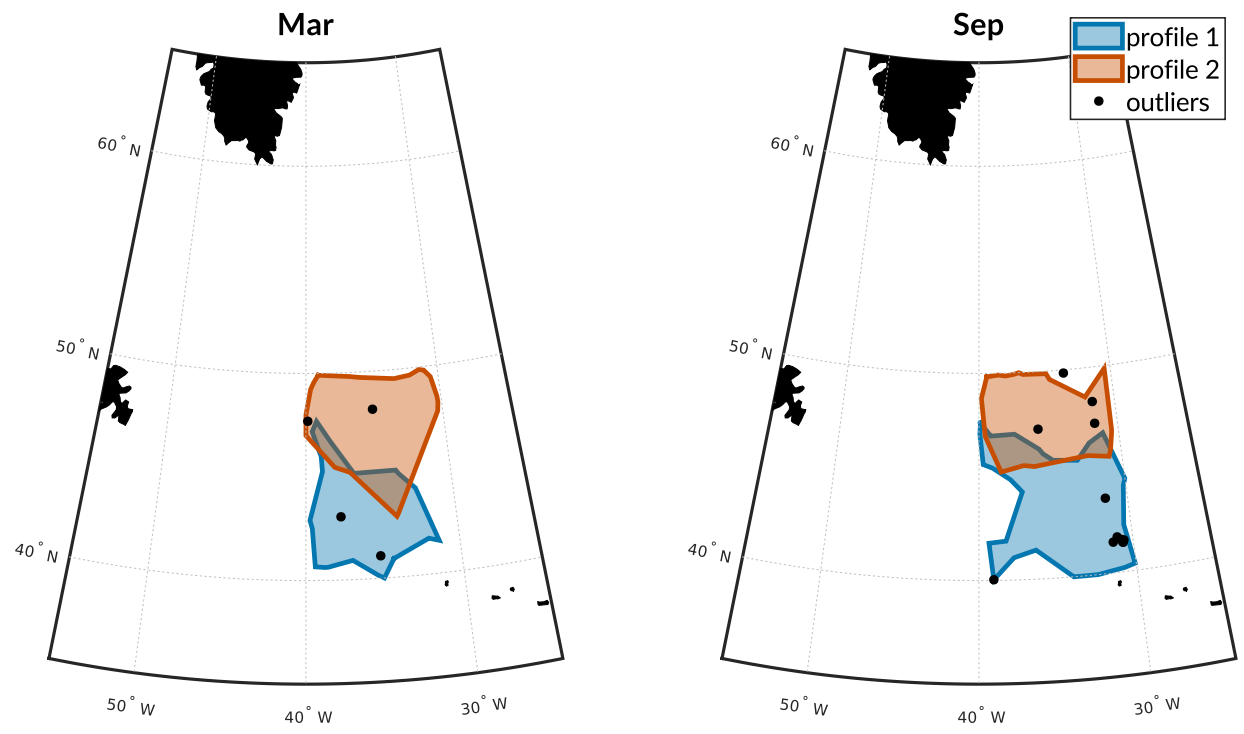

Figure 6.8: The spatial projection of previously found clusters in figure 6.7 in the Irminger Sea. 


\subsection{Bootstrapping Synthetic Benchmark Cases}

Bootstrapping is a technique to generate an ensemble of synthetic observations when there is limited data without making any assumptions about the underlying distribution or variances. ${ }^{15}$ While generally used to provide standard error and bias estimates or confidence intervals, the same basic procedure can be applied regardless of the statistic calculated. Given the principle of orthogonality in EOFs and their KDEs, we apply a bootstrapping technique to generate a new library of sound speed observations.

Figure 6.9 demonstrates the value of a bootstrapping approach for SSP generation. The previously saved KDEs are converted into inverse cumulative distribution functions, such that a random number generator can make an $\mathrm{N} x \mathrm{M}$ matrix of values from 0 to 1 , where $\mathrm{N}$ is the number of bootstrap iterations $(\mathrm{N}=1000)$, and $\mathrm{M}$ is the number of EOFs used $(\mathrm{M}=8)$. The random matrix, R, maps onto weights, W; the resulting N SSPs are simply the matrix product of the EOFs and W. The resulting SSPs, shown on the left hand side, are thus driven by the data collected for Irminger Box 3 without being limited to just those in the dataset.

Estimating depth-dependent navigation uncertainty is one natural application of bootstrapping. In this minimal working example, each bootstrapped SSP is run through a custom raytrace with a source depth at $50 \mathrm{~m}$, launch angles $-60: 5: 60$, and a bottom depth of $4000 \mathrm{~m}$. On the right hand side, the time-front for each ray is snapshotted for nominal OWTTs of 1-5 seconds and demarcated in a different color. For operational depths, generally the upper $250 \mathrm{~m}$, the width of any colored region begins to show an upper limit to ranging accuracy. This is a conservative estimate given that the bootstrapped SSPs are indifferent to the time of year. A more focused bootstrapping around a seasonal KDE would reduce the error observed.

\footnotetext{
${ }^{15}$ Hesterberg, "Bootstrap".
} 

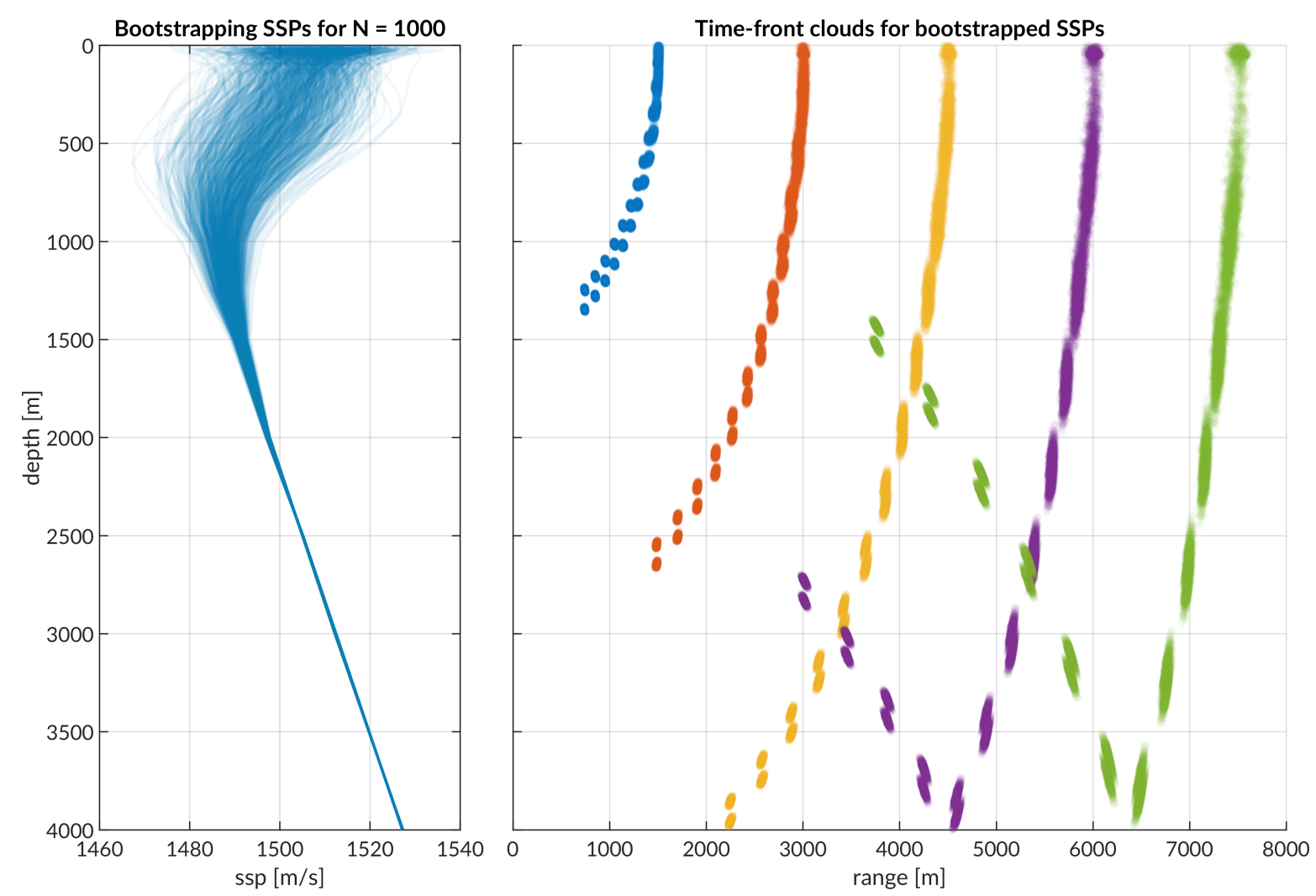

Figure 6.9: Bootstrapped SSPs (left) and their time-front ray clouds (right) for travel times of 1.0 (blue), 2.0 (orange), 3.0 (yellow), 4.0 (purple), and 5.0 (green) seconds. The source depth is $50 \mathrm{~m}$.

Of course, the bootstrapped SSPs can be used for any downstream metrics such as duct height and strength, depth-dependent mean and standard deviation transmission loss, ray shift from a CTD cast, or even to make acoustic climatologies. Further work will introduce these metrics into a bootstrapping pipeline and then isolate specific SSPs for virtual experiments based on these known metrics. Additional work is also needed in quantifying the joint probabilities between the bootstrapped KDEs and KDEs for any metric of interest, i.e., transmission loss at a single grid cell. That mapping would elucidate not only which EOFs drove acoustic features but how they varied throughout the water column. Bootstrapped methods also allow for a way to inject 
spatio-temporal variability into a field, i.e., through seeding an ocean with a dominant weight set and creating varying gridded fields based on differentials from the desired condition.

The transition from setting up virtual experiments with prescriptive data to synthetic data is one method of addressing that "big data" for SSPs tends to be orders of magnitude smaller than in other fields.

\subsection{Discussion}

In this chapter, we briefly overview the contributions to interfacing with the Virtual Ocean of the LAMSS/MOOS-IvP software project. The work in embedding modular SSP information, before and during an experiment, will have downstream impacts on acoustic communication, navigation, and sampling behavior. The automated pipeline to digest copious and disparate data from collaborators and output realistic, representative, and sometimes synthetic SSPs for further study allows useful references when developing autonomous behavior and signal processing

protocols. The realistic, data-driven benchmark studies can tease out the relationship between environmental and acoustic realism for Naval TDAs, ensuring that a suggested solution is not overly prescriptive or specific based on its environment. Furthermore, TDAs are a microcosm of decision making; their development and evaluation sets the stage for the next generation of autonomy on underwater vehicles. 


\section{Conccussion}

The ice was here, the ice was there,

The ice was all around:

It cracked and growled, and roared and howled,

Like noises in a swound!

Samuel Taylor Coleridge, The Rime of the Ancient Mariner (1834)

Embedded acoustic sensing on heterogenous platforms can create powerful networks of autonomous marine sensing systems. Successful coordination of fleets of vehicles will require consistent communication and precise navigation. In acoustically complex environments, robust embedded intelligence can maintain operator trust and open the door to a new set of sensing modalities. Collaborative AUVs can create modular synthetic aperture arrays; swim in concert for unprecedented sampling, with applications in estuary mapping, plume tracking, dye tracing; and monitor hard-to-reach regions of significant interest (like the Arctic) on consistent schedules and patterns. All of these applications require accurate and precise geo-referenced observations. As the easy integration of GPS spurred its application and relevance, we hope that the methodologies presented here-hardware and software-enable similar adoption and momentum in the underwater navigation and communication arena. 


\subsection{Summary of Contributions}

Current approaches in real-time underwater acoustic navigation simplify the non-linear relationship between a sound speed profile and acoustic propagation with a deterministic sound speed. Some post-processing approaches attempt to leverage the acoustic arrival structure via various ray methods, but often use a singular SSP for long-term deployments. Thus, the conversion from travel time to range, particularly for real-time vehicle deployments, can be preconditioned for error growth as the OWTT/range increases. This work addresses a critical need in acoustic navigation by retooling acoustic arrival methods generally deemed too complex or labor intensive for real-time, ray-based range estimation. This dissertation contributes an endto-end demonstration of how environmentally adaptive, real-time navigation can be succesfully deployed, from data assimilation to operational strategies to algorithms running onboard AUVs; furthermore, we do so in an acoustically complex environment in under-ice conditions, where the tolerance for error is close to none.

The heavy reliance on data, buttressed by models, to populate SSP training sets captures the widest variance possible; minimizes the data storage needs; facilitates the integration of near real-time results days before a field deployment; and simplifies understanding for roboticists who view oceanographic structure as a variable.

The TDA framework allows a human decision-maker to verify any SSP; quickly update the SSP based on local changes; and maintain trust in embedded vehicle intelligence. The decisionmaking approach is scalable for any region of interest and sustainable to implement as AUV deployments transition from from teams of humans supervising a single vehicle towards teams of vehicles collaborating with little human supervision. 
Lastly, the embedded stochastic prediction of a single group velocity is shown to be a smoothly varying function of range, source and receiver depth pairings, and multipath structure. The realtime system achieves GPS-like navigation without taking into account multipath structure; the ranging error improves by an order of magnitude with the suggested improvements, minimzing range error to single meters. Post-processing analysis shows that this method of ranging is actually sensitive to GPS drift.

These contributions are not possible without the software infrastructure, which the author's contributions build on decades of work by previous researchers affiliated with LAMSS.

\subsection{Future WORK}

Given that this work demonstrates an end-to-end system for real-time acoustic navigation for AUVs, there are many exciting avenues for future research.

\subsubsection{BeAufort Lens}

The Beaufort Lens will only become a more important feature of interest as the region continues to warm, and the physical and acoustical oceanographic communities build on each other's contributions to understanding the evolution and dynamics of the Beaufort Lens. However, an important piece of work will be a retrospective analysis using the Unified Database for Arctic and Subarctic Hydrography (UDASH). ${ }^{1}$ UDASH, which includes ITP data, has 288,532 oceanographic profiles for the subpolar seas north of $65^{\circ} \mathrm{N}$ for the period between 1980 and 2015, compared to the ITP record since 2006. The satellite ice extent record often displays a reference as the mean of years between 1980 to 2010; a more thorough examination of trends in sea ice extent,

\footnotetext{
${ }^{1}$ Behrendt et al., "UDASH - Unified Database for Arctic and Subarctic Hydrography".
} 
sea ice thickness, and duct strength can add potentially add evidence to the growing consensus that the subsurface warming has a first-order acceleration effect on sea ice melt.

Another important step forward will be in the unification of a single (or set) of duct parameters. The empirical nature of the duct parameter in this thesis is effective for a data assimilation pipeline, but it works in concert with the knee depth, bottom depth, and even temperature difference within the duct, to more holisitically convey the sound speed environment. This framing would benefit from a physically-driven measure of stability, akin to a buoyancy frequency, to describe the duct as well. Given the "big data" nature of analyzing duct variability, it is also crucial that these metrics be considered with respect to their impact on acoustic propagation.

\subsubsection{EOFs AND TDA FRAMEWORK}

The EOF method of compressing sound speed information, while a rich feature space, can certainly be improved. One direction is to directly include EOFs for salinity and temperature profiles, to decouple their effect on sound speed. Another is to apply machine learning methods, like dictionaries or autoencoders, to modify the feature space, or to shift the sound speed recreation mechanism from regression to classification.

One assumption in the current EOF method is applying the standard depth grid from HYCOM. This has already shown to impact environmental realism and acoustic utility as the depth spacing becomes sparser. Too fine of a depth grid may begin to encode unnecessary microstructure for the relevant acoustic effects. Thus, an algorithimic data-driven optimal depth grid may improve EOF performance by capturing the necessary resolution without over-interpolating existing data or over-fitting any desired sound speed. Additionally, training on a SSP data set reduces the ability to map sharp features or microstructure, many of which are important for 
acoustic propagation. Adding a parallel EOF set for the depth-dependent gradient of SSP may fill in the gap in estimating relevant microstructure or may even better represent SSP variability with a minimal amount of EOFs.

Gliders, often mentioned in this dissertation, could leverage a compressed statistical basis for SSP estimation for more effective low power communication by transmitting and/or receiving at tactically optimal depths. Any vehicle deployment, but gliders in particular, could be effective as through-the-sensor characterization of the SSP, using EOFs to both verify the SSP and communicate weights to other submerged platforms. An initial analysis of a through-the-sensor behavior for AUV Macrura during ICEX20 is available in Appendix B.. AUGs would also benefit from a spatially-aware EOF framework for depth-dependent current fields, to constrain hydrodynamic or inertial models and inform path planning.

The TDA framework, particularly the acoustic penalties, will be iterated on for further field testing. Importantly, acoustic communication is not directly represented by any metric. Folding in a metric like signal-to-noise ratio, soft decision error, ${ }^{2}$ or multi-path penalty ${ }^{3}$ will increase computational operational burden but provide a more tactically relevant acoustic decision space.

\subsubsection{Underwater NaVigation}

The underwater navigation solution presented in this thesis relies on many other elements in the ICNN. Particularly, for range estimation, the vehicle begins its mission with a GPS fix and the position differential is updated every 30 seconds. Thus, while the nearest bounce criteria is quite effective in isolating a single group velocity, more advanced autonomous behavior is necessary to distinguish when a simulated arrival time structure does not match the recorded OWTT. Virtual

\footnotetext{
${ }^{2}$ Preisig, "Performance analysis of adaptive equalization for coherent acoustic communications in the time-varying ocean environment".

${ }^{3}$ Schneider et al., "Self-Adapting Under-Ice Integrated Communications and Navigation Network".
} 
experiments may verify if and how the solution can converge (or not diverge) to an optimal group velocity given an initial erroneous range. Again, given large navigation error, machine learning techniques onboard the AUV could both shift the SSP weights and/or enlargen the simulation grid to pivot location and subselect an appropriate timefront.

This thesis makes the distinction between post-processing localization and real-time navigation results. The stochastic group velocity algorithm here is valid in both arenas and may need to be modified for mesoscale operations, to better account for range dependent propagation due to internal waves, eddies, or even bathymetric changes like underwater volcanoes. Furthermore, the nearest bounce criterion, in particular, leverages the upward refracting nature of the Arctic environment to separate multipath arrival by the number of (surface) bounces. This is not a "plug and play" technique for other acoustic propagation environments, where multipath is dominated by sound refraction, not reflection. However, BELLHOP simulations provide other relevant eigenray information, like time and angle of arrival, that is ripe for statistical and machine learning methods to classify a representative group velocity. A bespoke ray tracing method, like the one used in the TDA (Chapter 4), can easily report back the number of turning points instead of the number of surface bounces for multipath classification.

Lastly, the underwater navigation paradigm discussed will start to break down in extremely dynamic environments. Fast moving fronts, as seen in estuaries like the Connecticut River into the Long Island Sound, present an entirely new set of challenges not seen by internal waves or eddies. Previous AUV efforts ${ }^{4}$ in these regions simply dictate a trackline to follow. Even then, buoyancy flucutations heavily impact vehicle path planning and recovery. Acoustic communications are further complicated given a shallow environment with significant scattering due to

\footnotetext{
${ }^{4}$ author's fieldwork experience with A. Lavery, R. Geyer
} 
microstructure, ${ }^{5}$ where fast timefront modeling may only be coherent in low probabilities of the ocean state. Realistic in situ considerations of the acoustic environment may not be possible in such environments without complete through-the-sensor integration of echosounder data and/or a hyper-realistic onboard ocean model.

\subsubsection{COUPLED NAVIGATION AND TOMOGRAPHY}

Many approaches to underwater navigation combine it with acoustic tomography, i.e., a joint estimation of both source and receiver locations and the ocean volume between them. There has been considerable success at this effort in post-processing methods, which utilize intensive-and due to the non-linearity of sound propagation, often brute force-computational methods. For vehicle operations, fast tomography is the ideal implementation, in that one can fully consider how sound speed structure, horizontally and vertically, influences sound propagation. AUVs can serve as moving sources to better image the ocean volume, ${ }^{6}$ where mobile tomography and navigation converge on the same set of component technologies: position estimation, sound speed parameterization estimation, ray path identification, and vehicle path optimization.

But there are overwhelming challenges, operationally and computationally, for fast, mobile tomography to become a realistic endeavor. Addressing the spatial and temporal scales of what can be solved deterministically and what must be solved stochastically imposes a resolution constraint on the utility of gridded models that already dwarfs that of AUV operations. Given that AUV operations are often on small spatial and temporal scales, the added benefit of a gridded model is quite small, and in cases like the Arctic, may actually mischaracterize the ocean volume.

\footnotetext{
${ }^{5}$ Lavery et al., "Broadband acoustic quantification of stratified turbulence", "Measurements of acoustic scattering from zooplankton and oceanic microstructure using a broadband echosounder"; Ross et al., "Acoustic scattering from density and sound speed gradients: modeling of oceanic pycnoclines."

${ }^{6}$ Deffenbaugh, "Optimal Ocean Acoustic Tomography and Navigation with Moving Sources"; Elisseeff et al., "Ocean Acoustic Tomography as a Data Assimilation Problem".
} 
For gliders, with longer and larger operational scales, an ocean model may provide useful information. Currently gliders are low power and do not have the storage or computational power to run a full-scale, realistic ocean model. Of course, all of this is assuming that the ocean model is accurate and has high enough resolution to resolve ocean features at small enough scales, which is in of itself an active research field.

Ocean model realism (especially at finer scales) correlates with computational power and time. Thus, until a full-scale ocean model can be run in real-time on a small computer like a Raspberry $\mathrm{Pi}$, the framework proposed in this thesis strikes a balance for the necessary environmental realism as depth-dependent with temporal updates. This has a cascading effect on acoustic realism, currently a range-independent scheme. Faster, more effective computational methods (and hardware devices) for acoustic propagation can certainly improve the real-time joint estimation problem, but are still constrained by this depth-dependent snapshot of the ocean volume. Initial work in the Virtual Ocean can embed a 3D ocean model (or estimation of it), but the real-time acoustic propagation is limited to slices, not full 3D acoustic propagation.

\subsubsection{Oceanographic and Acoustic Observatories}

The solution presented in this thesis is a local positioning system; the accuracy provided would deteriorate as the receiver went further away from the beacons until the signal between them was no longer coherent. An accessible underwater equivalent to GNSS would catalyze oceanographic and naval research questions alike. But, what will it take for a global underwater navigation paradigm that rivals GNSS? Acoustic signals can travel far and wide when originating from a strategic depth, but crucially, they must be well positioned. Surface beacons, while easily located via GNSS, are liable to wave action and are easy targets for intentional and unintentional 
tampering. Bottom-mounted beacons are rigid and relatively tamper proof, but would be much more expensive to deploy accurately or fix. The frequency band for any acoustic communication would likely overlap with the sonic range of many animals. There are many exciting design questions ahead to make AUVs and GNSS a tool rather than a frontier.

One realistic step forward is to bake in positioning systems into acoustic and oceanographic observatories, ${ }^{7}$ like the one proposed for the Arctic. ${ }^{8}$ Sound in the ocean is an information dense medium; utilizing passive and active modalities, it is capable of observing ocean biology, geology, and physics. Thus, ocean observatories provide real-time updates and power to various tasks, including passive monitoring of biophony, geophony, and anthropogenic noise; acoustic remote sensing for tomography; active beacons for underwater navigation; and a whole host of relevant oceanographic observations. This effort requires significant infrastructure, but its development is on par (or less than) the material investment towards satellite infrastructure that drives GNSS.

The Arctic, a generally inaccessible, dangerous, and expensive region for crewed monitoring via icebreakers or vehicle deployments, is a prime location to invest in this type of sensing infrastructure. It is a region that is significantly undersampled, disproportionately so for its sensitivity to climate change and importance to the global heat and carbon budget. And it is a region, because of its instability, that is experiencing increased cultural, socioeconomic, and geopolitical relevance. The approach, methods, and results from this dissertation are a microcosm of what a multipurpose acoustic observatory could look like in the Arctic. As the oceanographic community is revolutionized by the co-evolution of big data, artificial intelligence, and autonomous platforms, I look forward to the progress we can make together to democratize more intelligent

\footnotetext{
${ }^{7}$ Howe et al., "Observing the Oceans Acoustically".

${ }^{8}$ Mikhalevsky et al., "Multipurpose Acoustic Networks in the Integrated Arctic Ocean Observing System".
} 
sensing and sampling, transitioning from cycles of fieldwork, analysis, and experiment design to data streams, interactive visualizations, and iteration.

\subsection{Closing comments}

Revisiting the quote at the beginning of the chapter, in the nineteenth century, the Arctic "captured the imagination and fueled the ambition of the Western world". ${ }^{9}$ Ships pursued the Northwest Passage, to accelerate trade; hunted whales, to garner a fortune; pined for glory, to lay claim to be furthest north. Yet what spurred the conquest of the Arctic was a, in hindsight, a cruelly ironic belief-a year-long, open, ice-free polar sea. Contemporary influential voices, from authors to cartographers to explorers, motivated this claim with a dizzying array of logic. Some cited natural phenomena like bird migration or the direction of ocean currents; others called on an omniscient Creator's plan or a lost paradise; and many wished to reignite geopolitical strength through imperial naval expansion. Our motivations for studying the Arctic-scientific to aweinspired to geopolitical and tactical-have not changed that much.

Our motivations for ocean acoustics have a similar complicated history, intertwining military strength and natural observations. In 1912, shortly after the Titanic sunk, the first patent for underwater echo ranging system was filed, "to detect the presence of large objects underwater". During World War I, this idea birthed the Allied Submarine Detection Information Committee (ASDIC) and Sound Navigation and Ranging (SONAR). Experiments in the echo ranging led to the discovery of the "afternoon effect" by Columbus Iselin, where sonar performance degrades in the afternoon of calm sunny days due to a temperature increase at the top of the mixed layer,

\footnotetext{
${ }^{9}$ Schulz, "Literature's Arctic Obsession".
} 
and the invention of the bathythermograph by Athelstan Spilhaus, a small torpedo-shaped device that detects temperature changes versus pressure.

By World War II, the bathythermograph was modified by Allyn Vine and installed on the outer hulls of US submarines. But the majority of research in ocean acoustics during the war effort was antisubmarine warfare, as German U-boats had considerable success sinking merchant ships in American waters and even deployed acoustic mines. ${ }^{10}$ Doc Ewing and Joe Worzel sailed all over the east coast setting off explosives and acquiring data to understand acoustics on continental shelves; they saw ground and water waves, as well as dispersive features of the broadband pulse in shallow water.

Indeed, research in WWII and the Cold War set the stage for modern ocean acoustics. High frequency acoustics was introduced as it was most relevant to the sonars used to locate submarines and mines. Noise analysis was introduced as background noise impedes sonar performance. Low frequency acoustics was introduced as a potential mechanism to locate a downed aircraft or sinking ship, where a released explosive charge could be picked up by listening stations. While this never happened due to pilots not wanting explosives on their plane (entirely reasonable), it was successfully tested off the Bahamas with four pounds of TNT charges. These long distance transmissions provided the foundation for the Sound Fixing and Ranging (SOFAR) project, which now names the channel it exploits. The Office of Naval Research (ONR) retooled SOFAR and funded the American Telephone and Telegraph Company (AT\&T) to develop an undersea surveillance system of arrays of hydrophones at the bottom of the ocean to detect Soviet submarines. These were particularly successful in tracking the noisy diesel and then nuclear So-

\footnotetext{
${ }^{10}$ Impressively, acoustic mines can be tracked as far back to the 14 th century military treatise, Huolongjing, of the early Ming Dynasty.
} 
viet submarines, but also picked up the sounds of "sea monsters", later determined to be blue and fin whales.

This history goes on and on, and is a fascinating rabbit hole that provides incredible and necessary context for where we are today. Many methods, sampling devices, and motivating questions originate from extremely complex, technically interdisciplinary, and applied problems for military use. Today, when warming by anthropogenic climate change recalibrates national security with international climate security, we perhaps have a new, unifying motivation - to better observe and steward this planet we call home. 
Appendix 


\section{A. GPS DRIFT DURING ICEX20}
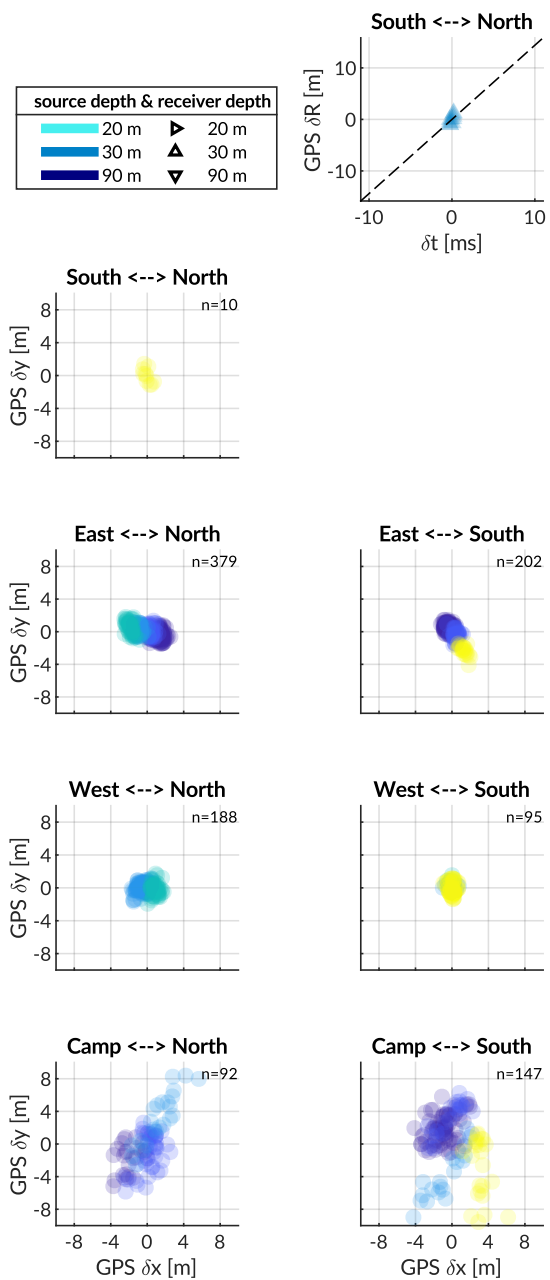
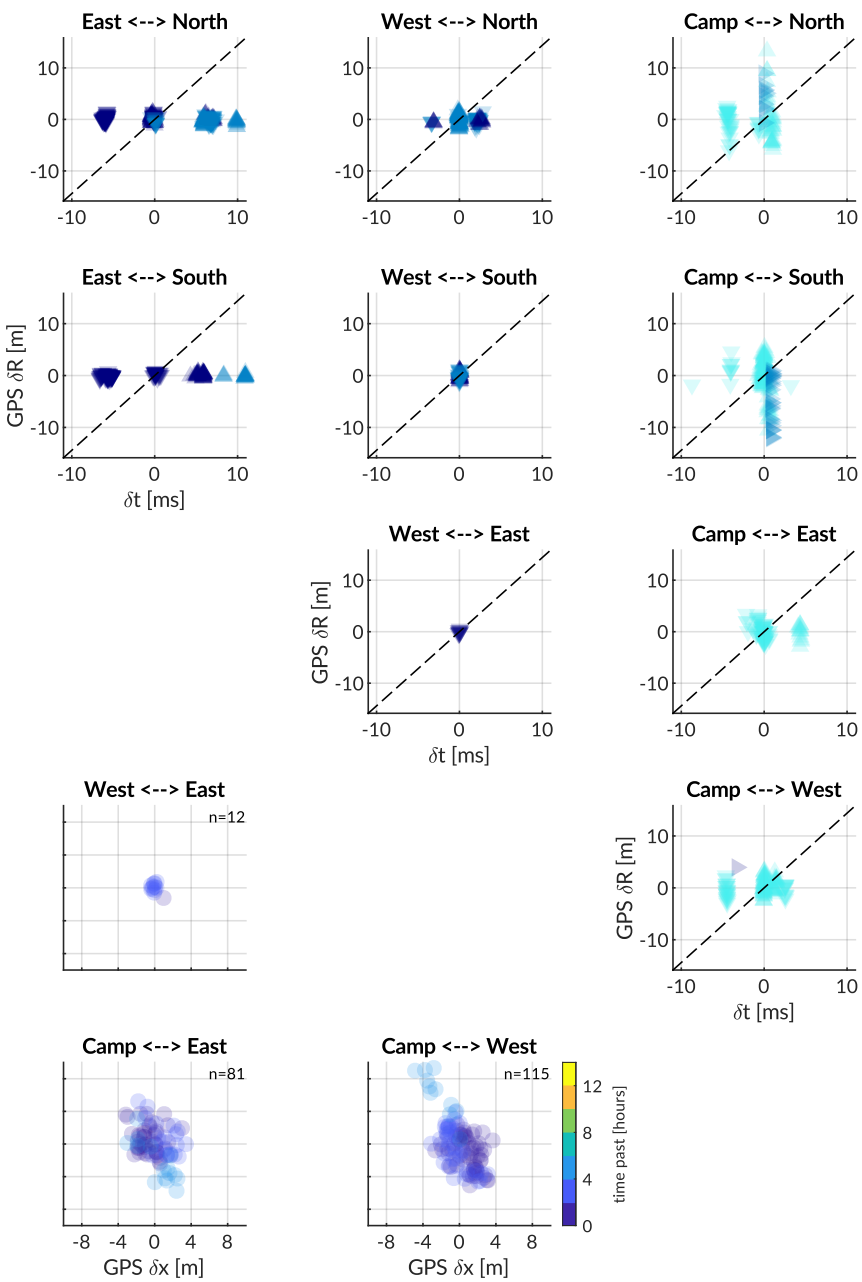

Figure 1: The upper diagonal shows GPS drift against OWTT spread demarcated by beacon to beacon connections. The lower diagonal shows GPS drift in $\mathrm{x}$ and $\mathrm{y}$ colored by hours since the beginning of the beacon experiment. This is a comprehensive comparison compared to the subset provided in figure 5.17 .

The bottom diagonal characterizes the amount of GPS drift seen at polar latitudes. The GPS at Camp is particularly noisy due to the human infrastructure and lack of open sky over the GPS puck. Transforming the $\mathrm{x}$ - and $\mathrm{y}$ - drift into range drift for the upper diagonal, the pattern of GPS drift dominating OWTT drift is apparent across all node connections. Some travel time spreads show distinct clusters of arrivals, which represent multipath conditions and/or different source-receiver depths, not systemic errors in the OWTT measurement. The full scope of results further supports the fact that the acoustic ranging method, while not always as accurate as GPS, is more precise. 


\section{B. Through the sensor characterization of the Beaufort}

\section{LENS FROM A/V MACRURA}
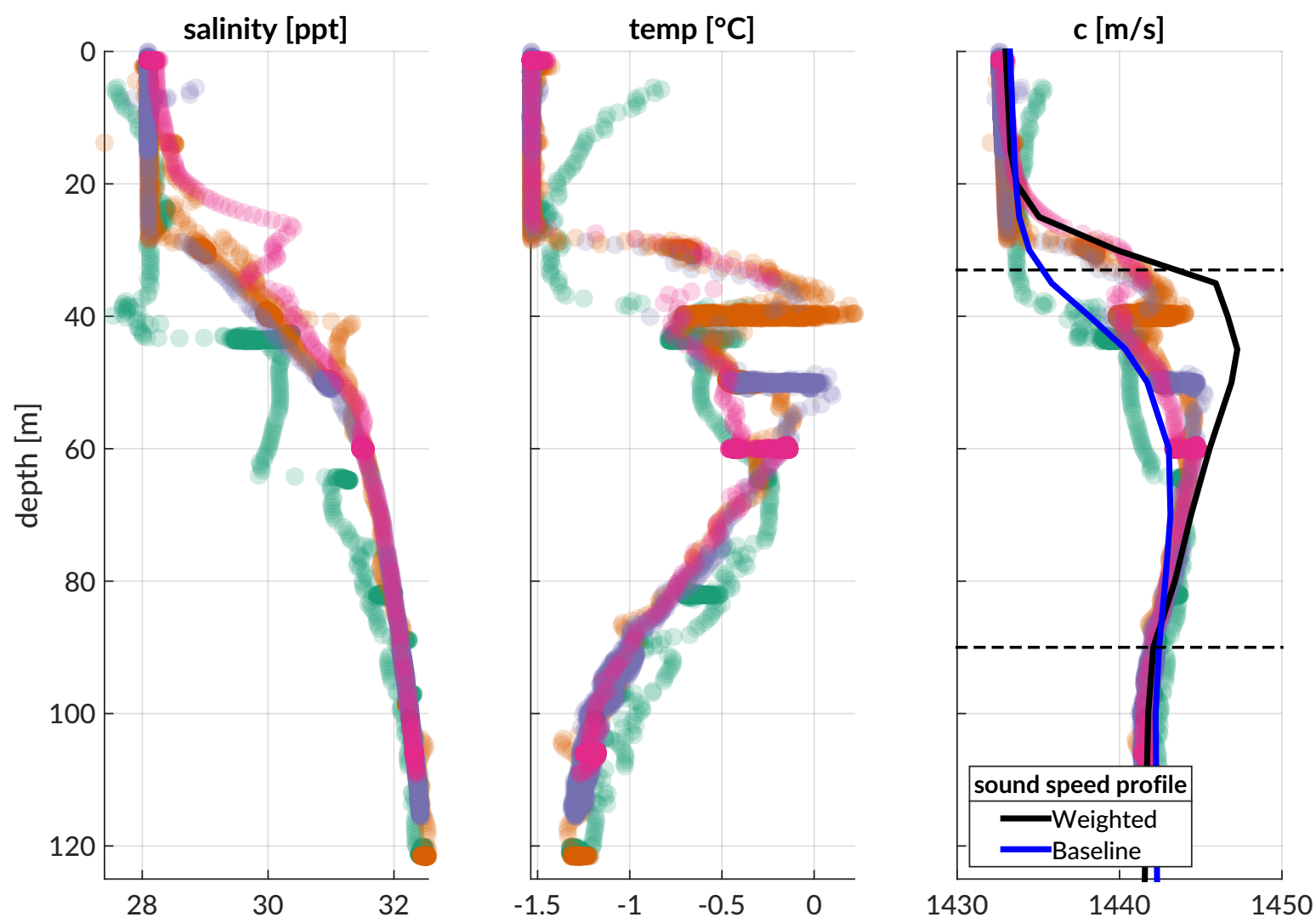

Figure 2: Through the sensor CTD information, separated by individual upward and downward transects, compared to the EOF sound speed baseline and weighted conditions. The left two plots show the AUV's observed salinity and temperature profiles, respectively. The rightmost plot shows the calculated sound speed profile.

It is important to note that any through-the-sensor characterization of an SSP begins to violate the "snapshot" paradigm in both space and time. A clear instance of this violation (vertically) occurs when the AUV spends a disproportionately longer amount of time at the same depth, such as $40 \mathrm{~m}$. The horizontal ambiguity derives from the AUV yoyo-ing through an ocean volume; indeed, the desired sound speed is not perfectly recreated by any of the data accrued. This introduces unwanted complexity in comparing the effectiveness of through-the-sensor characterization to some "known" SSP. Future work should focus on both environmental- and acoustic-driven tweaking of a previously established SSP for enhanced performance. 


\section{RUNNING AN EOF MISSION IN LAMSS}

The focus of this section is to demonstrate how to run and interact with an example LAMSS mission that uses the environmental EOF framework. In particular, we will run through a sample mission in the Arctic.

Again, this appendix assumes you have a working LAMSS repository checked out and installed on your computer. To verify this make sure that the following executables are built and findable in your shell path. If these commands do not work, check out the guide at lamss/src/doc/moos_at_lamssV2.

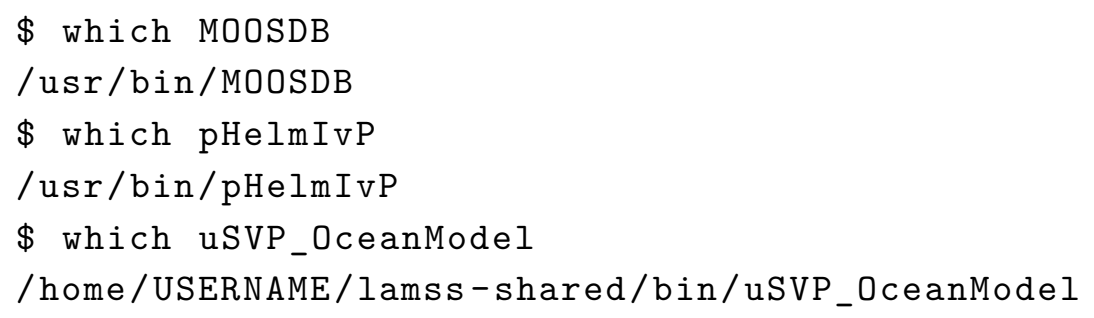

\section{C1 LAUNCHING THE SCRIPT}

Go to your missions-lamss directory and run this particular script.

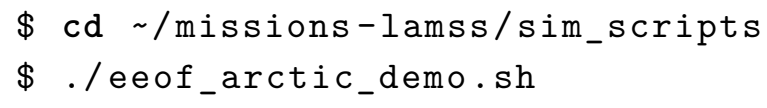

A series of windows will open and the vehicle will begin running a default pattern. If all is working well, you will see three screens of pMarineViewer - one for Camp Sargo and two for AUV Macrura (the vehicle and its autonomy). Depending on your MATLAB configuration, you may also start to see some transmission loss plots. 


\section{C2 Simulation interaction via Goby Liaison}

In order to interact with the simulation we will use Goby Liaison. Using your favorite web browser, navigate to the Goby Liaison address. If running everything on the same machine, navigate to this address: localhost: 50001. If running from a remote system from the simulation, then navigate using the simulation machine’s IP address: http://192.168.170. 205:50001. Of course, please make sure to change the IP address as appropriate. We will see the following example web page.

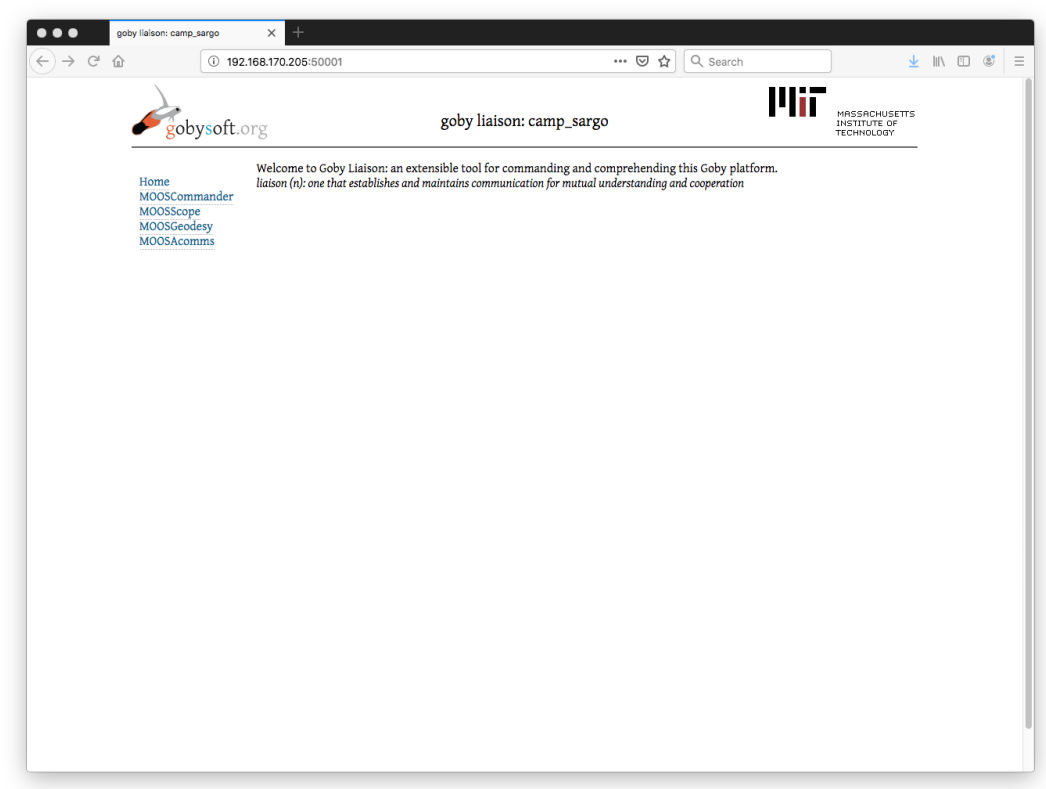

Figure 3: The Goby Liaison is a portal that allows for vehicle interaction.

The following figures will walk through the steps to send an EOF update via the parameterized, bounded message, LAMSS_ENVIRONMENT_UPDATE.

It is useful to use the screen command to scope a MOOS process. For example, to see how the Virtual Ocean is running on the AUV, enter the following:

\$ screen -r macrura.uSVP_OceanModel 
$\mathrm{C}$

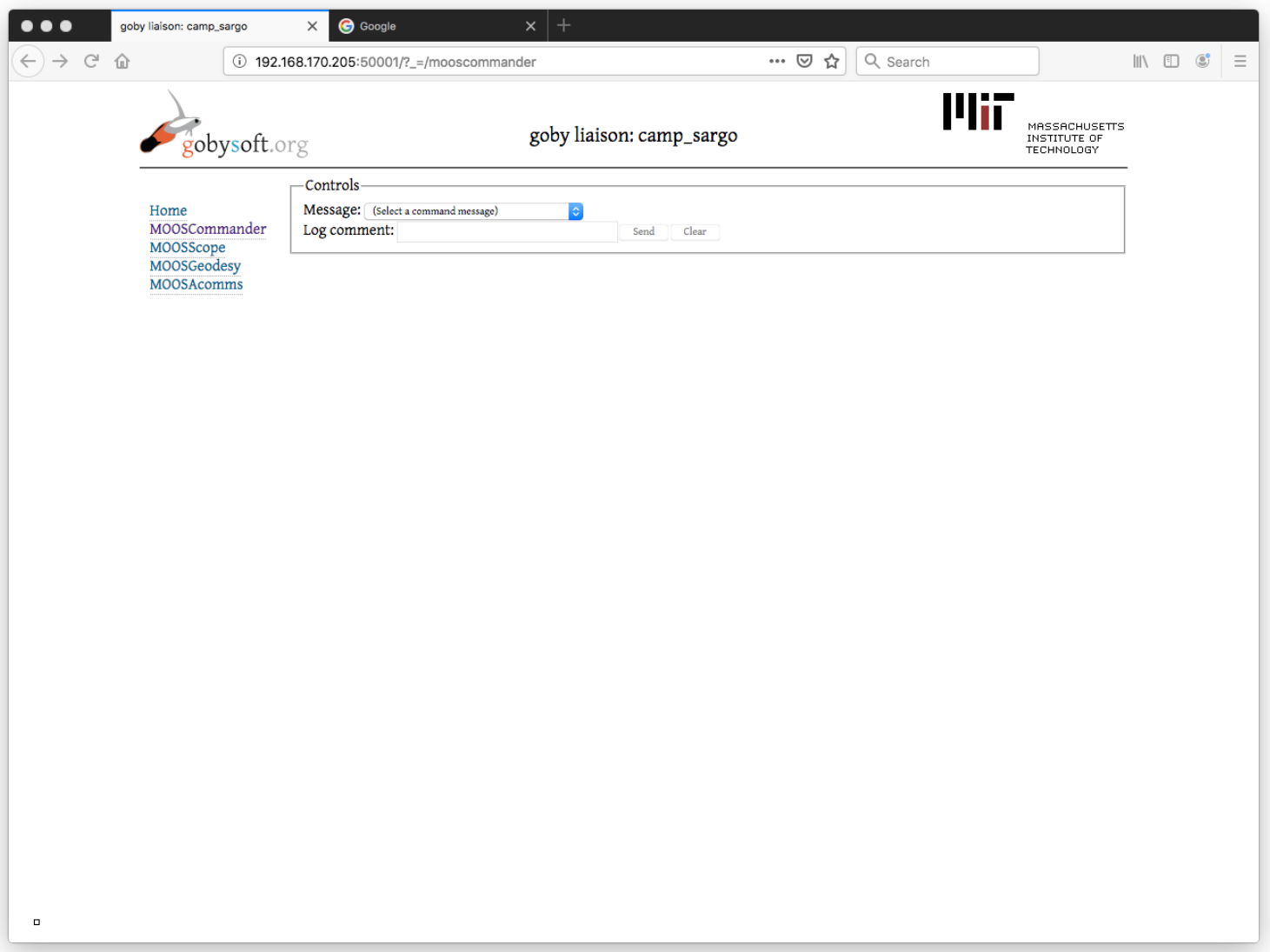

Figure 4: This is the initial view of the Goby Commander. It allows you to choose what message you would like to send. Advanced users can script a message in advance to bypass this manual system.

You can detach a screen output using ctrl-a d. Using ctrl-c will kill the process on the simulated vehicle. 


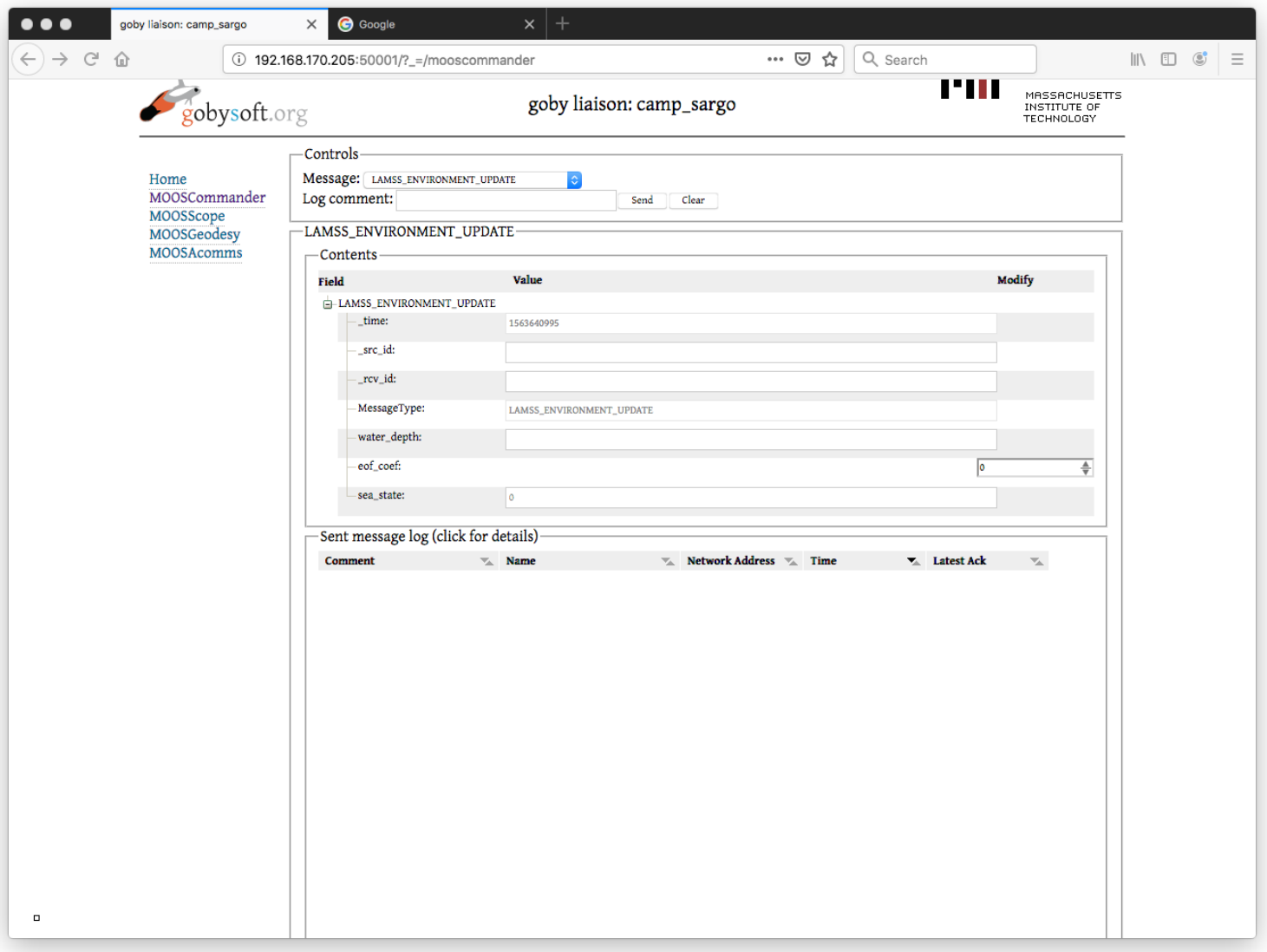

Figure 5: Once you choose to send an environmental update, the Goby Commander displays the relevant fields. The message fields are designed for a range independent environment; there are further message types for the expanded internal wave capabilities. 


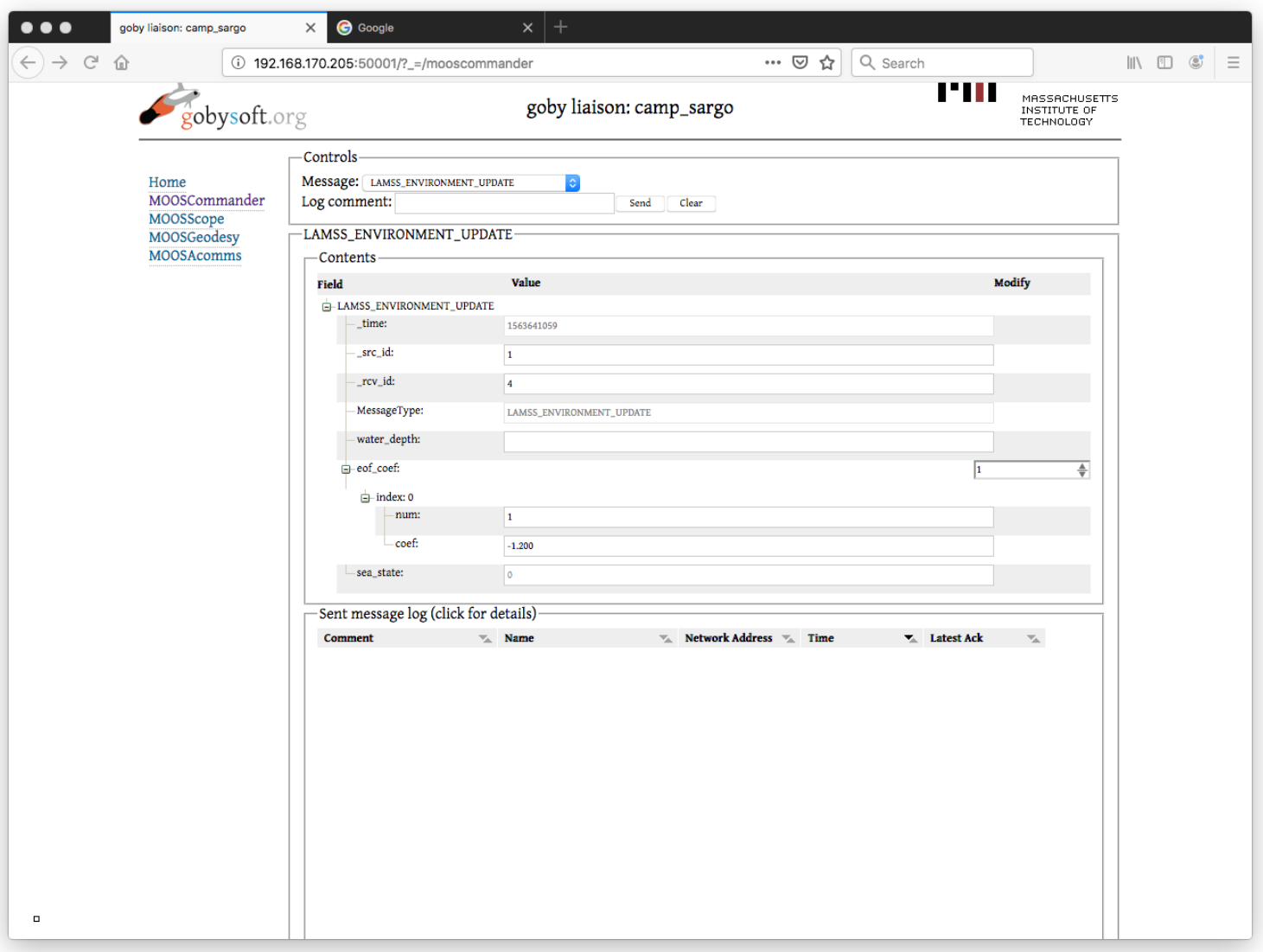

Figure 6: Here we send a basic message. We select the first EOF weight to be updated with a value of 1.200. Notice that the source modem ID is 1 and the vehicle's modem ID is 4 . The dialog next to eof_coef increases how many are sent in the same message. 


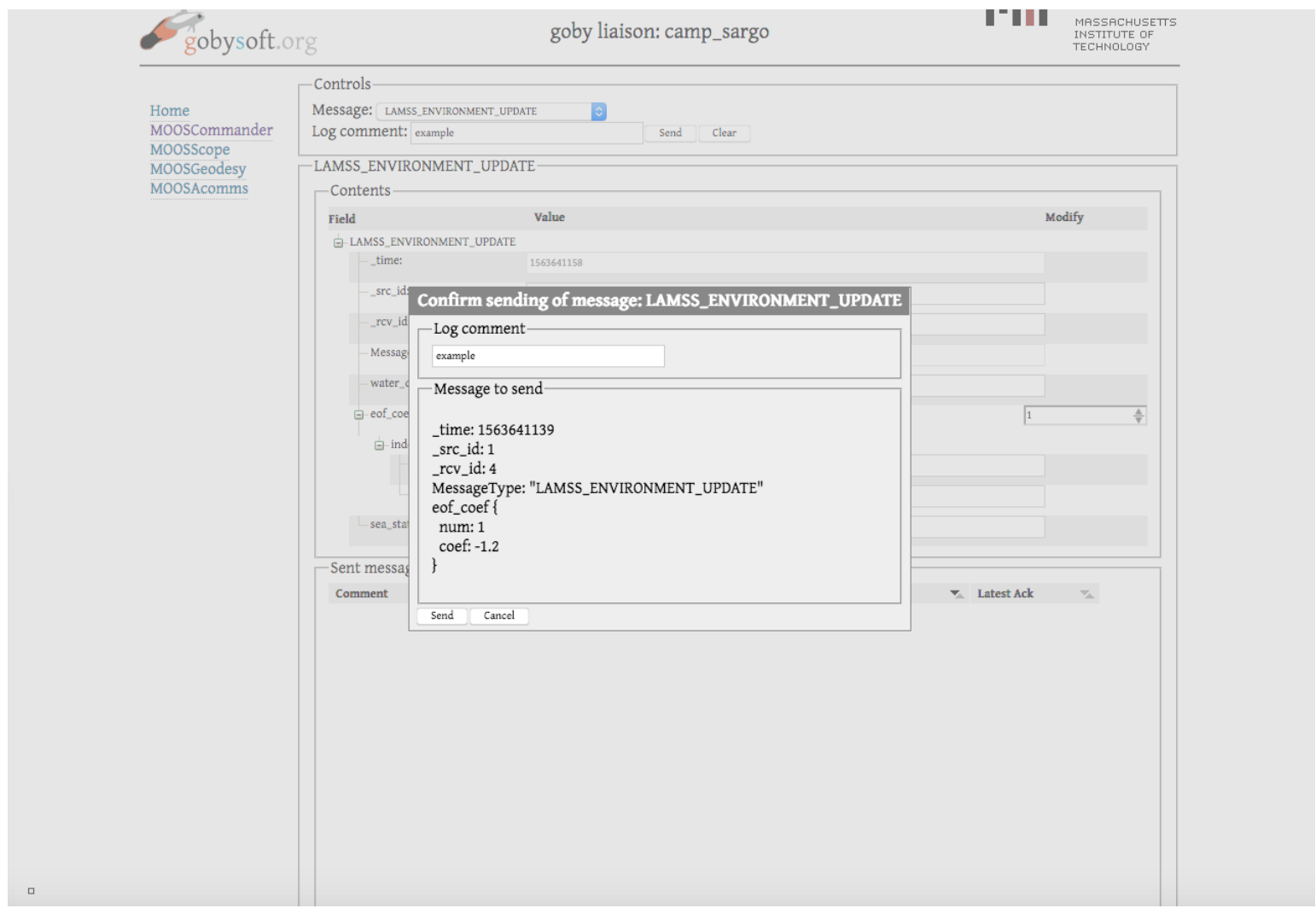

Figure 7: The Goby Commander will confirm that this is indeed the message you wish to send and provides a space where you can log any comments. 


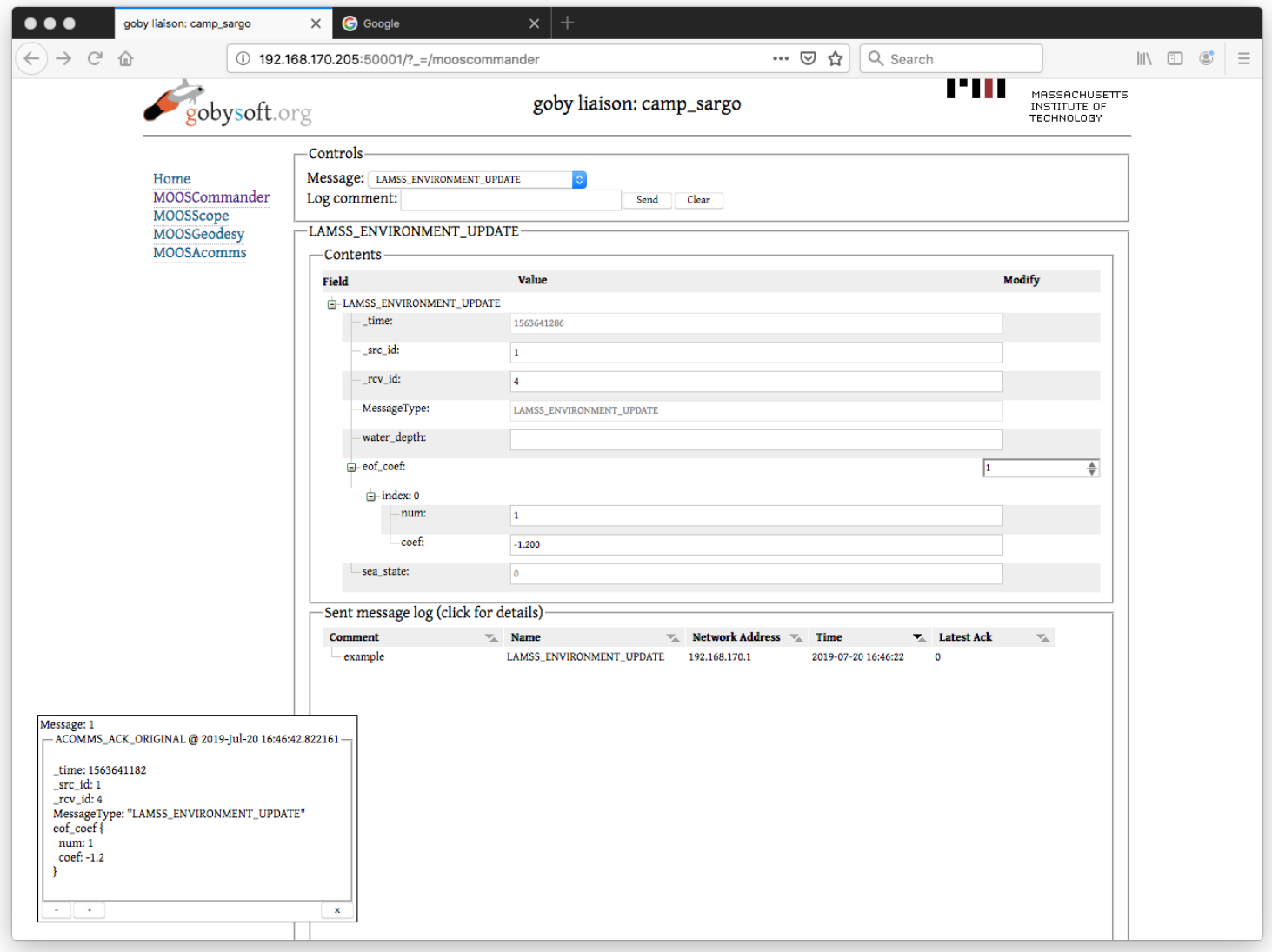

Figure 8: The Goby Commander will acknowledge the successful receipt of the message from the vehicle. 


\section{BiBLIOGRAPHY}

1. Ainslie, M. A. \& McColm, J. G. A simplified formula for viscous and chemical absorption in sea water. The Journal of the Acoustical Society of America 103. doi:10 .1121/1. 421258 (1998).

2. Aksenov, Y., Karcher, M., Proshutinsky, A., Gerdes, R., Cuevas, B. d., Golubeva, E., Kauker, F., Nguyen, A. T., Platov, G. A., Wadley, M., Watanabe, E., Coward, A. C. \& Nurser, A. J. G. Arctic pathways of Pacific Water: Arctic Ocean Model Intercomparison experiments. Journal of Geophysical Research: Oceans 121. doi:https : //doi .org/10 . 1002/2015JC011299 (2016).

3. Badiey, M., Wan, L., Pecknold, S. \& Turgut, A. Azimuthal and temporal sound fluctuations on the Chukchi continental shelf during the Canada Basin Acoustic Propagation Experiment 2017. The Journal of the Acoustical Society of America 146. doi:10.1121/1.5141373 (2019).

4. Ballard, M. S., Badiey, M., Sagers, J. D., Colosi, J. A., Turgut, A., Pecknold, S., Lin, Y.-T., Proshutinsky, A., Krishfield, R., Worcester, P. F. \& Dzieciuch, M. A. Temporal and spatial dependence of a yearlong record of sound propagation from the Canada Basin to the Chukchi Shelf. The Journal of the Acoustical Society of America 148. doi:10 . 1121/10 . 0001970 (2020).

5. Barker, L. D. L., Jakuba, M. V., Bowen, A. D., German, C. R., Maksym, T., Mayer, L., Boetius, A., Dutrieux, P. \& Whitcomb, L. L. Scientific Challenges and Present Capabilities in Underwater Robotic Vehicle Design and Navigation for Oceanographic Exploration UnderIce. Remote Sensing 12. doi:10 .3390/rs12162588 (2020).

6. Behrendt, A., Sumata, H., Rabe, B. \& Schauer, U. UDASH - Unified Database for Arctic and Subarctic Hydrography. Earth System Science Data 10. doi:10 . 5194 / essd-10-11192018 (2018).

7. Bellingham, J., Leonard, J., Vaganay, J., Goudey, C., Atwood, D., Consi, T., Bales, J., Schmidt, H. \& Chryssostomidis, C. AUV Operations in the Arctic (1993).

8. Bellingham, J. G. \& Rajan, K. Robotics in Remote and Hostile Environments. Science 318. doi:10.1126/science.1146230 (2007).

9. Benjamin, M. R., Schmidt, H., Newman, P. M. \& Leonard, J. J. Nested autonomy for unmanned marine vehicles with MOOS-IvP. Journal of Field Robotics 27. doi:https : / doi . org/10.1002/rob. 20370 (2010). 
10. Bianco, M. \& Gerstoft, P. Dictionary learning of sound speed profiles. The Journal of the Acoustical Society of America 141. doi:10 .1121/1. 4977926 (2017).

11. Bogue, R. Underwater robots: a review of technologies and applications. Industrial Robot: An International Journal 42. doi:10 .1108/IR-01-2015-0010 (2015).

12. Brown, N. \& Mueller, C. Designing With Data: Moving Beyond The Design Space Catalog (2017).

13. Brugler, E. T., Pickart, R. S., Moore, G. W. K., Roberts, S., Weingartner, T. J. \& Statscewich, H. Seasonal to interannual variability of the Pacific water boundary current in the Beaufort Sea. Progress in Oceanography 127. doi:10.1016/j . pocean. 2014.05 .002 (2014).

14. Carper, S. A. Low frequency active sonar performance in the Arctic Beaufort Lens MA thesis (Massachusetts Institute of Technology and Woods Hole Oceanographic Institution, Woods Hole, MA, 2017). doi:10 .1575/1912/9249.

15. Casagrande, G., Stephan, Y., Varnas, A. C. W. \& Folegot, T. A Novel Empirical Orthogonal Function (EOF)-Based Methodology to Study the Internal Wave Effects on Acoustic Propagation. IEEE Journal of Oceanic Engineering 36. doi:10 . 1109/ JOE . 2011 . 2161158 (2011).

16. Catipovic, J., Deffenbaugh, M., Freitag, L. \& Frye, D. An acoustic telemetry system for deep ocean mooring data acquisition and control in Proceedings OCEANS 3 (1989). doi:10.1109/ OCEANS . 1989.586702.

17. Chamberlain, P. M., Talley, L. D., Mazloff, M. R., Riser, S. C., Speer, K., Gray, A. R. \& Schwartzman, A. Observing the Ice-Covered Weddell Gyre With Profiling Floats: Position Uncertainties and Correlation Statistics. Journal of Geophysical Research: Oceans 123. doi:https : / doi org/10.1029/2017JC012990 (2018).

18. Chapin, F. S., Sturm, M., Serreze, M. C., McFadden, J. P., Key, J. R., Lloyd, A. H., McGuire, A. D., Rupp, T. S., Lynch, A. H., Schimel, J. P., Beringer, J., Chapman, W. L., Epstein, H. E., Euskirchen, E. S., Hinzman, L. D., Jia, G., Ping, C.-L., Tape, K. D., Thompson, C. D. C., Walker, D. A. \& Welker, J. M. Role of Land-Surface Changes in Arctic Summer Warming. Science 310. doi:10.1126/science.1117368 (2005).

19. Chassignet, E. P., Hurlburt, H. E., Smedstad, O. M., Halliwell, G. R., Hogan, P. J., Wallcraft, A. J., Baraille, R. \& Bleck, R. The HYCOM (HYbrid Coordinate Ocean Model) data assimilative system. Journal of Marine Systems. Marine Environmental Monitoring and Prediction 65. doi:10.1016/j . jmarsys . 2005.09.016 (2007).

20. Chen, C.-T. \& Millero, F. J. Speed of sound in seawater at high pressures. The Journal of the Acoustical Society of America 62 (1977).

21. Chen, R., Poulsen, A. \& Schmidt, H. Spectral, spatial, and temporal characteristics of underwater ambient noise in the Beaufort Sea in 1994 and 2016. The Journal of the Acoustical Society of America 145. doi:10.1121/1.5088601 (2019). 
22. Chen, R. \& Schmidt, H. Temporal and spatial characteristics of the Beaufort Sea ambient noise environment. The Journal of the Acoustical Society of America 148. doi:10.1121/10 . 0002955 (2020).

23. Claus, B., Kepper, J. H., Suman, S. \& Kinsey, J. C. Closed-loop one-way-travel-time navigation using low-grade odometry for autonomous underwater vehicles. Journal of Field Robotics 35. doi:https : / doi .org/10.1002/rob. 21746 (2018).

24. Collyer, S. C. \& Malecki, G. S. Tactical decision making under stress: History and overview Washington, DC, US, 1998.

25. Corlett, W. B. \& Pickart, R. S. The Chukchi slope current. Progress in Oceanography 153. doi:10.1016/j . pocean. 2017.04.005 (2017).

26. Cornuelle, B., Munk, W. \& Worcester, P. Ocean acoustic tomography from ships. Journal of Geophysical Research: Oceans 94. doi:https : //doi .org/10 .1029/JC094iC05p06232 (1989).

27. Cornuelle, B. D. Inverse methods and results from the 1981 ocean acoustic tomography experiment (1983).

28. Cross, J. N., Mathis, J. T., Pickart, R. S. \& Bates, N. R. Formation and transport of corrosive water in the Pacific Arctic region. Deep Sea Research Part II: Topical Studies in Oceanography. Synthesis of Arctic Research SOAR Phase II 152. doi:10 . 1016 / j . dsr2 . 2018 . 05 . 020 (2018).

29. Das, J., Py, F., Maughan, T., O’Reilly, T., Messié, M., Ryan, J., Rajan, K. \& Sukhatme, G. S. Simultaneous tracking and sampling of dynamic oceanographic features with autonomous underwater vehicles and lagrangian drifters in (Springer Verlag, 2014). doi:10 . 1007/978-3642-28572-1_37.

30. Davis, W. D., Holmberg, D., Reneke, P., Brassell, L. \& Vettori, R. Demonstration of real-time tactical decision aid displays 2007.

31. Deffenbaugh, M., Bellingham, J. \& Schmidt, H. The relationship between spherical and hyperbolic positioning in OCEANS 96 MTS/IEEE Conference Proceedings. The Coastal Ocean Prospects for the 21st Century 2(1996). doi:10.1109/OCEANS . 1996.568293.

32. Deffenbaugh, M., Schmidt, H. \& Bellingham, J. Acoustic navigation for Arctic under-ice AUV missions in Proceedings of OCEANS '93 (1993). doi:10 .1109/OCEANS . 1993. 326007.

33. Deffenbaugh, M., Schmidt, H. \& Bellingham, J. Acoustic positioning in a fading multipath environment in OCEANS 96 MTS/IEEE Conference Proceedings. The Coastal Ocean - Prospects for the 21st Century 2 (1996). doi:10.1109/OCEANS . 1996.568294.

34. Deffenbaugh, M. Optimal Ocean Acoustic Tomography and Navigation with Moving Sources $\mathrm{PhD}$ Thesis (Massachusetts Institute of Technology, 1997). 
35. Del Grosso, V.A. New equation for the speed of sound in natural waters (with comparisons to other equations). The Journal of the Acoustical Society of America 56. doi:10 .1121/1. 1903388 (1974).

36. Del Grosso, V.A. \& Mader, C. W. Speed of Sound in Pure Water. The Journal of the Acoustical Society of America 52. doi:10.1121/1.1913258 (1972).

37. Dickey, T., Lewis, M. \& Chang, G. Optical oceanography: Recent advances and future directions using global remote sensing and in situ observations. Reviews of Geophysics 44. doi:https: //doi org/10.1029/2003RG000148 (2006).

38. Dickey, T. The Role of New Technology in Advancing Ocean Biogeochemical Research. Oceanography 14. doi:10.5670/oceanog. 2001.11 (2001).

39. Duarte, C. M., Chapuis, L., Collin, S. P., Costa, D. P., Devassy, R. P., Eguiluz, V. M., Erbe, C., Gordon, T. A. C., Halpern, B. S., Harding, H. R., Havlik, M. N., Meekan, M., Merchant, N. D., Miksis-Olds, J. L., Parsons, M., Predragovic, M., Radford, A. N., Radford, C. A., Simpson, S. D., Slabbekoorn, H., Staaterman, E., Opzeeland, I. C. V., Winderen, J., Zhang, X. \& Juanes, F. The soundscape of the Anthropocene ocean. Science 371. doi:10 . 1126 / science. aba4658 (2021).

40. Duda, T. F., Zhang, W. G. \& Lin, Y.-T. Effects of Pacific Summer Water layer variations and ice cover on Beaufort Sea underwater sound ducting. Journal of the Acoustical Society of America (2021).

41. Duda, T. F., Zhang, W. G., Lin, Y.-T. \& Newhall, A. E. Long-range sound propagation in the Canada Basin in Underwater Acoustics 2019 (2019).

42. Duda, T. F. Acoustic signal and noise changes in the Beaufort Sea Pacific Water duct under anticipated future acidification of Arctic Ocean waters. The Journal of the Acoustical Society of America 142. doi:10.1121/1.5006184 (2017).

43. Duda, T. F., Morozov, A. K., Howe, B. M., Brown, M. G., Speer, K., Lazarevich, P., Worcester, P. F. \& Cornuelle, B. D. Evaluation of a Long-Range Joint Acoustic Navigation / Thermometry System in (2006). doi:10 .1109/0CEANS . 2006 . 306999.

44. Duhan, D. Tactical Decision Aid for Unmanned Vehicles in Maritime Missions (2005).

45. Dushaw, B. D., Worcester, P. F., Cornuelle, B. D. \& Howe, B. M. On equations for the speed of sound in seawater. The Journal of the Acoustical Society of America 93 (1993).

46. Elisseeff, P., Schmidt, H. \& Xu, W. Ocean Acoustic Tomography as a Data Assimilation Problem. IEEE Journal of Oceanic Engineering 27 (2002).

47. Eustice, R. M., Whitcomb, L. L., Singh, H. \& Grund, M. Recent Advances in SynchronousClock One-Way-Travel-Time Acoustic Navigation in OCEANS 2006 (2006). doi:10 . 1109 / OCEANS . 2006.306931.

48. Fetterer, F., Knowles, K., Meier, W., Savoie, M. \& Windnagel, A. Sea Ice Index, Version 3 2017. 
49. Fossum, T. O., Eidsvik, J., Ellingsen, I., Alver, M. O., Fragoso, G. M., Johnsen, G., Mendes, R., Ludvigsen, M. \& Rajan, K. Information-driven robotic sampling in the coastal ocean. Journal of Field Robotics 35. doi:10. 1002/rob . 21805 (2018).

50. Freitag, L., Ball, K., Partan, J., Koski, P. \& Singh, S. Long range acoustic communications and navigation in the Arctic in OCEANS 2015 - MTS/IEEE Washington (2015). doi:10 . 23919/ OCEANS . 2015.7401956.

51. Gaillard, F. Ocean acoustic tomography with moving sources or receivers. Journal of Geophysical Research: Oceans 90. doi:https : / / doi .org/10 . 1029 / JC090iC06p11891 (1985).

52. Gardner, A. \& Collins, J. A second look at Chip Scale Atomic Clocks for long term precision timing. OCEANS 2016 MTS/IEEE Monterey. doi:10 . 1109/OCEANS . 2016. 7761268 (2016).

53. Grasso, R. \& Giannecchini, S. Geo-spatial Tactical Decision Aid systems: fuzzy logic for supporting decision making in 2006 9th International Conference on Information Fusion (2006). doi:10.1109/ICIF . 2006.301754.

54. Graupe, C. E., van Uffelen, L. J., Webster, S. E., Worcester, P. F. \& Dzieciuch, M. A. Preliminary results for glider localization in the Beaufort Duct using broadband acoustic sources at long range in Oceans 2019 MTS/IEEE Seattle (2019). doi:10 . 23919/OCEANS40490 . 2019. 8962637.

55. Gwal, A. K. \& Jain, A. GPS scintillation studies in the arctic region during the first winterphase 2008 Indian Arctic Expedition. Polar Science 4. doi:10 . 1016/j · polar . 2010 . 08. 001 (2011).

56. Haley, P. J. \& Lermusiaux, P. F. J. Multiscale two-way embedding schemes for free-surface primitive equations in the "Multidisciplinary Simulation, Estimation and Assimilation System”. Ocean Dynamics 60. doi:10 . 1007/s 10236-010-0349-4 (2010).

57. Helber, R. W., Barron, C. N., Dastugue, J. M. \& Toner, M. S. The Acoustic Parameter Climatology. US Navy Journal of Underwater Acoustics 64 (2015).

58. Helber, R. W., Townsend, T. L., Barron, C. N., Dastugue, J. M. \& Carnes, M. R. Validation Test Report for the Improved Synthetic Ocean Profile (ISOP) System, Part I: Synthetic Profile Methods and Algorithm: 2013.

59. Hesterberg, T. Bootstrap. WIREs Computational Statistics 3. doi:https : //doi . org/10 . 1002/wics. 182 (2011).

60. Hover, F. S. Path planning for data assimilation in mobile environmental monitoring systems in Proceedings of the 2009 IEEE/RSJ international conference on Intelligent robots and systems (IEEE Press, St. Louis, MO, USA, 2009). 
61. Howe, B. M., Miksis-Olds, J., Rehm, E., Sagen, H., Worcester, P. F. \& Haralabus, G. Observing the Oceans Acoustically. Frontiers in Marine Science 6. doi:10 . 3389 /fmars . 2019. 00426 (2019).

62. Huang, A., Olson, E. \& Moore, D. LCM: Lightweight Communications and Marshalling in (2010). doi:10.1109/IROS. 2010.5649358.

63. Jakuba, M. V., Roman, C. N., Singh, H., Murphy, C., Kunz, C., Willis, C., Sato, T. \& Sohn, R. A. Long-baseline acoustic navigation for under-ice autonomous underwater vehicle operations. Journal of Field Robotics 25. doi:https : / / doi .org/10 . 1002/rob . 20250 (2008).

64. Jensen, F. B., Kuperman, W. A., Porter, M. B. \& Schmidt, H. Computational Ocean Acoustics (Springer New York, New York, NY, 2011).

65. Jensen, J. K., Hjelmervik, K. T. \& Ostenstad, P. Finding Acoustically Stable Areas Through Empirical Orthogonal Function (EOF) Classification. IEEE Journal of Oceanic Engineering 37. doi:10.1109/JOE . 2011.2168669 (2012).

66. Jin, Y., Moen, J. I. \& Miloch, W. J. GPS scintillation effects associated with polar cap patches and substorm auroral activity: direct comparison. Journal of Space Weather and Space Climate 4. doi:10 .1051/swsc/2014019 (2014).

67. Johnson, B. \& Treadway, W. A. Artificial Intelligence - An Enabler of Naval Tactical Decision Superiority. AI Magazine 40. doi:10 .1609/aimag. v40i1. 2852 (2019).

68. Jung, T. S., Hegel, T. M., Bentzen, T. W., Egli, K., Jessup, L., Kienzler, M., Kuba, K., Kukka, P. M., Russell, K., Suitor, M. P. \& Tatsumi, K. Accuracy and performance of low-feature GPS collars deployed on bison Bison bison and caribou Rangifer tarandus. Wildlife Biology 2018. doi:10.2981/wlb.00404 (2018).

69. Kaminski, C., Crees, T., Ferguson, J., Forrest, A., Williams, J., Hopkin, D. \& Heard, G. 12 days under ice - an historic AUV deployment in the Canadian High Arctic in 2010 IEEE/OES Autonomous Underwater Vehicles ISSN: 2377-6536 (2010). doi:10 . 1109 / AUV . 2010 . 5779651.

70. Kanungo, T., Mount, D., Netanyahu, N., Piatko, C., Silverman, R. \& Wu, A. An efficient k-means clustering algorithm: analysis and implementation. IEEE Transactions on Pattern Analysis and Machine Intelligence 24. doi:10 . 1109/TPAMI . 2002 . 1017616 (2002).

71. Kepper, J. H., Claus, B. C. \& Kinsey, J. C. MEMS IMU and One-Way-Travel-Time Navigation for Autonomous Underwater Vehicles in Oceans 2017 - Aberdeen (Aberdeen, UK, 2017).

72. Khurshudyan, I. \& Freedman, A. An oil spill in Russia's Arctic exposes problems in Moscow's big plans for the Far North 2020.

73. Kilfoyle, D. \& Baggeroer, A. The state of the art in underwater acoustic telemetry. IEEE Journal of Oceanic Engineering 25. doi:10.1109/48. 820733 (2000). 
74. Krishfield, R., Toole, J., Proshutinsky, A. \& Timmermans, M.-L. Automated Ice-Tethered Profilers for Seawater Observations under Pack Ice in All Seasons. Journal of Atmospheric and Oceanic Technology 25. doi:10.1175/2008JTECH0587 . 1 (2008).

75. Krupskii, P., Huser, R. \& Genton, M. G. Factor Copula Models for Replicated Spatial Data. Journal of the American Statistical Association 113. doi:10 . 1080/01621459 . 2016. 1261712 (2018).

76. Kucukosmanoglu, M., Colosi, J. A., Worcester, P. F., Dzieciuch, M. A. \& Torres, D. J. Observations of sound-speed fluctuations in the Beaufort Sea from summer 2016 to summer 2017. The Journal of the Acoustical Society of America 149. doi:10.1121/10.0003601 (2021).

77. Kukulya, A., Plueddemann, A., Austin, T., Stokey, R., Purcell, M., Allen, B., Littlefield, R., Freitag, L., Koski, P., Gallimore, E., Kemp, J., Newhall, K. \& Pietro, J. Under-ice operations with a REMUS-100 AUV in the Arctic in 2010 IEEE/OES Autonomous Underwater Vehicles ISSN: 2377-6536 (2010). doi:10 .1109/AUV . 2010.5779661.

78. Kukulya, A., Bellingham, J., Kaeli, J., Reddy, C., Godin, M. \& Conmy, R. Development of a propeller driven long range autonomous underwater vehicle (LRAUV) for under-ice mapping of oil spills and environmental hazards: An Arctic Domain Center of Awareness project (ADAC) in 2016 IEEE/OES Autonomous Underwater Vehicles (AUV) ISSN: 2377-6536 (2016). doi:10 . 1109/AUV . 2016.7778655.

79. Kunz, C., Murphy, C., Camilli, R., Singh, H., Bailey, J., Eustice, R., Jakuba, M., Nakamura, K.-i., Roman, C., Sato, T., Sohn, R. A. \& Willis, C. Deep sea underwater robotic exploration in the ice-covered Arctic ocean with AUVs in 2008 IEEE/RSJ International Conference on Intelligent Robots and Systems (2008). doi:10.1109/IROS . 2008.4651097.

80. Lam, F. P. A., Haley, P. J., Janmaat, J., Lermusiaux, P. F. J., Leslie, W. G., Schouten, M. W., Te Raa, L. A. \& Rixen, M. At-sea real-time coupled four-dimensional oceanographic and acoustic forecasts during Battlespace Preparation 2007. Journal of Marine Systems 78. doi:10 . 1016/j . jmarsys . 2009.01.029 (2009).

81. Lavery, A. C., Geyer, W. R. \& Scully, M. E. Broadband acoustic quantification of stratified turbulence. The Journal of the Acoustical Society of America 134. doi:10.1121/1. 4807780 (2013).

82. Lavery, A. C., Chu, D. \& Moum, J. N. Measurements of acoustic scattering from zooplankton and oceanic microstructure using a broadband echosounder. ICES Journal of Marine Science 67. doi:10.1093/icesjms/fsp242 (2010).

83. LeBlanc, L. R. \& Middleton, F. H. An underwater acoustic sound velocity data model. The Journal of the Acoustical Society of America 67. doi:10 .1121/1. 384448 (1980).

84. Lermusiaux, P. F. J. \& Chiu, C. Four Dimensional Data Assimilation for Coupled PhysicalAcoustical Fields (1996). 
85. Lin, Y., Newhall, A. E., Duda, T. F., Lermusiaux, P. F. J. \& Haley, P. J. Merging MultiplePartial-Depth Data Time Series Using Objective Empirical Orthogonal Function Fitting. IEEE Journal of Oceanic Engineering 35. doi:10 . 1109/ JOE . 2010.2052875 (2010).

86. Lustgarten, A. The Great Climate Migration Has Begun. The New York Times (2020).

87. MacDougall, A. H., Avis, C. A. \& Weaver, A. J. Significant contribution to climate warming from the permafrost carbon feedback. Nature Geoscience 5. doi:10 . 1038/ngeo1573 (2012).

88. Mackenzie, K. V. Nine-term equation for sound speed in the oceans. The Journal of the Acoustical Society of America 70. doi:10.1121/1.386920 (1981).

89. MacKinnon, J. A., Simmons, H. L., Hargrove, J., Thomson, J., Peacock, T., Alford, M. H., Barton, B. I., Boury, S., Brenner, S. D., Couto, N., Danielson, S. L., Fine, E. C., Graber, H. C., Guthrie, J., Hopkins, J. E., Jayne, S. R., Jeon, C., Klenz, T., Lee, C. M., Lenn, Y.-D., Lucas, A. J., Lund, B., Mahaffey, C., Norman, L., Rainville, L., Smith, M. M., Thomas, L. N., Torres-Valdés, S. \& Wood, K. R. A warm jet in a cold ocean. Nature Communications 12. doi:10.1038/s41467-021-22505-5 (2021).

90. Mathis, J. T., Cooley, S. R., Lucey, N., Colt, S., Ekstrom, J., Hurst, T., Hauri, C., Evans, W., Cross, J. N. \& Feely, R. A. Ocean acidification risk assessment for Alaska's fishery sector. Progress in Oceanography. Synthesis of Arctic Research (SOAR) 136. doi:10 . 1016 / j . pocean.2014.07.001 (2015).

91. McFarlane, J. R. \& Mackay, L. Autonomous Underwater Vehicle operations in the Arctic in OCEANS 2015 - MTS/IEEE Washington (2015). doi:10 . 23919/OCEANS . 2015 . 7404510.

92. Melo, J. \& Matos, A. Towards LBL positioning systems for multiple vehicles in OCEANS 2016 - Shanghai (2016). doi:10.1109/OCEANSAP . 2016.7485619.

93. Mikhalevsky, P. N., Sagen, H., Worcester, P. F., Baggeroer, A. B., Orcutt, J., Moore, S. E., Lee, C. M., Vigness-Raposa, K. J., Freitag, L., Arrott, M., Atakan, K., Beszczynska-Möller, A., Duda, T. F., Dushaw, B. D., Gascard, J. C., Gavrilov, A. N., Keers, H., Morozov, A. K., Munk, W. H., Rixen, M., Sandven, S., Skarsoulis, E., Stafford, K. M., Vernon, F. \& Yuen, M. Y. Multipurpose Acoustic Networks in the Integrated Arctic Ocean Observing System. ARCTIC 68. doi:10 . 14430/arctic4449 (2015).

94. Mikhalevsky, P. N., Sperry, B. J., Woolfe, K. F., Dzieciuch, M. A. \& Worcester, P. F. Deep ocean long range underwater navigation. The Journal of the Acoustical Society of America 147. doi:10.1121/10.0001081 (2020).

95. Mitchell, C. N., Alfonsi, L., Franceschi, G. D., Lester, M., Romano, V. \& Wernik, A. W. GPS TEC and scintillation measurements from the polar ionosphere during the October 2003 storm. Geophysical Research Letters 32. doi:https : / / doi . org / 10 . 1029 / 2004GL021644 (2005). 
96. Moen, R., Pastor, J., Cohen, Y. \& Schwartz, C. C. Effects of Moose Movement and Habitat Use on GPS Collar Performance. The Journal of Wildlife Management 60. doi:10 . 2307 / 3802085 (1996).

97. Moore, A. M., Martin, M. J., Akella, S., Arango, H. G., Balmaseda, M., Bertino, L., Ciavatta, S., Cornuelle, B., Cummings, J., Frolov, S., Lermusiaux, P., Oddo, P., Oke, P. R., Storto, A., Teruzzi, A., Vidard, A. \& Weaver, A. T. Synthesis of ocean observations using data assimilation for operational, real-time and reanalysis systems: A more complete picture of the state of the ocean. Frontiers in Marine Science 6. doi:10 . 3389 / fmars . 2019 . 00090 (2019).

98. Naranjo, L. The modern sea ice satellite record turns 402018.

99. Newhall, A. E., Lin, Y.-T., Lynch, J. F., Baumgartner, M. F. \& Gawarkiewicz, G. G. Long distance passive localization of vocalizing sei whales using an acoustic normal mode approach. The Journal of the Acoustical Society of America 131. doi:10 .1121/1. 3666015 (2012).

100. Newman, P. \& Leonard, J. Pure range-only sub-sea SLAM in 2003 IEEE International Conference on Robotics and Automation (Cat. No.03CH37422) 2 (2003). doi:10 . 1109 / ROBOT . 2003.1241875.

101. Nishimura, C. Monitoring whales and earthquakes by using SOSUS. (Naval Research Laboratory Review, 1994).

102. Norgren, P., Lubbad, R. \& Skjetne, R. Unmanned underwater vehicles in Arctic operations in (2014).

103. Novitzky, M., Dougherty, H. R. R. \& Benjamin, M. R. A Human-Robot Speech Interface for an Autonomous Marine Teammate in Social Robotics (2016). doi:10 . 1007 / 978-3-31947437-3_50.

104. Novitzky, M., Robinette, P., Benjamin, M. R., Gleason, D. K., Fitzgerald, C. \& Schmidt, H. Preliminary Interactions of Human-Robot Trust, Cognitive Load, and Robot Intelligence Levels in a Competitive Game in Companion of the 2018 ACM/IEEE International Conference on Human-Robot Interaction (2018). doi:10 .1145/3173386.3177000.

105. Paull, L., Saeedi, S., Seto, M. \& Li, H. AUV Navigation and Localization: A Review. IEEE Journal of Oceanic Engineering 39. doi:10 . 1109/ JOE. 2013.2278891 (2014).

106. Petillo, S. Autonomous \& Adaptive Oceanographic Feature Tracking On Board Autonomous Underwater Vehicles. OCEANS 2015 - Genova. doi:10 . 1109/OCEANS-Genova . 2015. 7271616 (2014).

107. Petillo, S., Balasuriya, A. \& Schmidt, H. Autonomous Adaptive Environmental Assessment and Feature Tracking via Autonomous Underwater Vehicles in Oceans 2010 IEEE - Sydney (2010). 
108. Petillot, Y. R., Antonelli, G., Casalino, G. \& Ferreira, F. Underwater Robots: From Remotely Operated Vehicles to Intervention-Autonomous Underwater Vehicles. IEEE Robotics Automation Magazine 26. doi:10 .1109/MRA . 2019 . 2908063 (2019).

109. Plueddemann, A. J., Kukulya, A. L., Stokey, R. \& Freitag, L. Autonomous Underwater Vehicle Operations Beneath Coastal Sea Ice. IEEE/ASME Transactions on Mechatronics 17. Conference Name: IEEE/ASME Transactions on Mechatronics. doi:10 . 1109/TMECH . 2011.2174798 (2012).

110. Pollitt, G. W. Mine Countermeasures (MCM) Tactical Decision Aids (TDAs), A Historical Review. Military Operations Research 11 (2006).

111. Porter, M. B. The KRAKEN Normal Mode Program 1992.

112. Porter, M. B. The BELLHOP Manual and User's Guide: Preliminary Draft 2011.

113. Poulsen, A. J. \& Schmidt, H. Acoustic noise properties in the rapidly changing Arctic Ocean in (Buenos Aires, Argentina, 2016). doi:10.1121/2.0000552.

114. Preisig, J. Acoustic propagation considerations for underwater acoustic communications network development. ACM SIGMOBILE Mobile Computing and Communications Review 11. doi:10.1145/1347364.1347370 (2007).

115. Preisig, J. C. Performance analysis of adaptive equalization for coherent acoustic communications in the time-varying ocean environment. The Journal of the Acoustical Society of America 118. doi:10.1121/1.1907106 (2005).

116. Putland, R. L., Mackiewicz, A. G. \& Mensinger, A. F. Localizing individual soniferous fish using passive acoustic monitoring. Ecological Informatics 48. doi:10 . 1016/ j . ecoinf . 2018.08.004 (2018).

117. Quigley, M., Gerkey, B., Conley, K., Faust, J., Foote, T., Leibs, J., Berger, E., Wheeler, R. \& $\mathrm{Ng}$, A. ROS: an open-source Robot Operating System (2009).

118. Randeni, S., Schneider, T. \& Schmidt, H. Construction of a high-resolution under-ice AUV navigation framework using a multidisciplinary virtual environment in (2020). doi:10 . 1109/ AUV50043.2020.9267950.

119. Randeni, S., Schneider, T., Schmidt, H., Bhatt, E. \& Viquez, O. A high-resolution AUV navigation framework with integrated communication and tracking for under-ice deployments. Field Robotics. (in review) (2021).

120. Reid, T., Walter, T., Blanch, J. \& Enge, P. GNSS Integrity in The Arctic. NAVIGATION 63. doi:https://doi.org/10.1002/navi.169(2016).

121. Robinson, A. R. \& Lermusiaux, P. F. Prediction Systems With Data Assimilation for Coupled Ocean Science and Ocean Acoustics. Theoretical and Computational Acoustics 2003 The Sixth International Conference (ICTCA). doi:10 .1142/9789812702609_0031 (2004). 
122. Ross, T. \& Lavery, A. C. Acoustic scattering from density and sound speed gradients: modeling of oceanic pycnoclines. The Journal of the Acoustical Society of America 131. doi:10 . 1121/1. 3669394 (2012).

123. Rossby, T., Dorson, D. \& Fontaine, J. The RAFOS System. Journal of Atmospheric and Oceanic Technology 3. doi:10 .1175/1520-0426 (1986) 003<0672: TRS>2 . 0 . C0 ; 2 (1986).

124. Rossby, T. \& Webb, D. Observing abyssal motions by tracking Swallow floats in the SOFAR channel. Deep Sea Research and Oceanographic Abstracts 17. doi:10 . 1016/0011-7471 (70) 90027-6 (1970).

125. Rypkema, N. R. Underwater \& Out of Sight: Towards Ubiquity in Underwater Robotics (2019).

126. Schmidt, H. OASES Version 3.1 User guide and reference manual 2011.

127. Schmidt, H. \& Schneider, T. Acoustic Communication and Navigation in the New ArcticA Model Case for Environmental Adaptation. Underwater Communications and Networking (UComms) (2016).

128. Schneider, T. \& Schmidt, H. A State Observation Technique for Highly Compressed Source Coding of Autonomous Underwater Vehicle Position. IEEE Journal of Oceanic Engineering 38. doi:10.1109/JOE. 2013.2268292 (2013).

129. Schneider, T. \& Schmidt, H. Model-Based Adaptive Behavior Framework for Optimal Acoustic Communication and Sensing by Marine Robots. IEEE Journal of Oceanic Engineering 38. doi:10 . 1109/JOE. 2012. 2232492 (2013).

130. Schneider, T. \& Schmidt, H. NETSIM: A Realtime Virtual Ocean Hardware-in-the-loop Acoustic Modem Network Simulator in 2018 Fourth Underwater Communications and Networking Conference (UComms) (2018). doi:10 .1109/UComms . 2018. 8493188.

131. Schneider, T. \& Schmidt, H. The Dynamic Compact Control Language: A compact marshalling scheme for acoustic communications in OCEANS'10 IEEE SYDNEY (IEEE, Sydney, Australia, 2010). doi:10 .1109/OCEANSSYD . 2010.5603520.

132. Schneider, T. \& Schmidt, H. Unified command and control for heterogeneous marine sensing networks. Journal of Field Robotics 27. doi:https : / / doi . org/10 . 1002/rob . 20346 (2010).

133. Schneider, T., Schmidt, H. \& Randeni, S. Self-Adapting Under-Ice Integrated Communications and Navigation Network (2020).

134. Schneider, T. E. Advances in Integrating Autonomy with Acoustic Communications for Intelligent Networks of Marine Robots (2013).

135. Schofield, O., Kohut, J., Aragon, D., Creed, L., Graver, J., Haldeman, C., Kerfoot, J., Roarty, H., Jones, C., Webb, D. \& Glenn, S. Slocum Gliders: Robust and ready. Journal of Field Robotics 24. doi:https: //doi .org/10.1002/rob. 20200 (2007). 
136. Schulz, K. Literature's Arctic Obsession. The New Yorker (2017).

137. Seger, K. D. \& Miksis-Olds, J. L. Acoustic documentation of temperate odontocetes in the Bering and Chukchi Seas. Marine Mammal Science 35. doi:https : //doi .org/10 .1111/ mms. 12577 (2019).

138. Sherman, J., Davis, R., Owens, W. \& Valdes, J. The autonomous underwater glider "Spray”. IEEE Journal of Oceanic Engineering 26. doi:10 .1109/48. 972076 (2001).

139. Singh, S., Grund, M., Bingham, B., Eustice, R., Singh, H. \& Freitag, L. Underwater Acoustic Navigation with the WHOI Micro-Modem in OCEANS 2006 (IEEE, Boston, MA, USA, 2006). doi:10.1109/0CEANS . 2006.306853.

140. Steinfeld, A., Fong, T., Kaber, D., Lewis, M., Scholtz, J., Schultz, A. \& Goodrich, M. Common metrics for human-robot interaction in Proceeding of the 1st ACM SIGCHI/SIGART conference on Human-robot interaction - HRI '06 (ACM Press, Salt Lake City, Utah, USA, 2006). doi:10 . 1145/1121241.1121249.

141. Stevens, L. A., Straneo, F., Das, S. B., Plueddemann, A. J., Kukulya, A. L. \& Morlighem, M. Linking glacially modified waters to catchment-scale subglacial discharge using autonomous underwater vehicle observations. The Cryosphere 10. doi:10 .5194/tc-10417-2016 (2016).

142. Stojanovic, M. \& Preisig, J. Underwater acoustic communication channels: Propagation models and statistical characterization. IEEE Communications Magazine 47. doi:10 . 1109/ MCOM. 2009 . 4752682 (2009).

143. Stommel, H. The Slocum Mission. Oceanography 2. doi:10 . 5670 / oceanog . 1989 . 26 (1989).

144. Swanlund, D., Maraj, R., Schuurman, N., Hope, R., Donkers, K., Aquin, M. \& Rickerby, G. Gps performance in yukon's arctic coast. Geografiska Annaler: Series A, Physical Geography 98. doi:10.1111/geoa. 12143 (2016).

145. Themens, D. R., Jayachandran, P. T. \& Langley, R. B. The nature of GPS differential receiver bias variability: An examination in the polar cap region. Journal of Geophysical Research: Space Physics 120. doi:https : //doi .org/10. 1002/2015JA021639 (2015).

146. Thyng, K. M., Greene, C. A., Hetland, R. D., Zimmerle, H. M. \& DiMarco, S. F. True colors of oceanography: Guidelines for effective and accurate colormap selection. Oceanography 29. doi:10.5670/oceanog. 2016.66 (2016).

147. Tiemann, C. O., Porter, M. B. \& Frazer, L. N. Localization of marine mammals near Hawaii using an acoustic propagation model. The Journal of the Acoustical Society of America 115. doi:10.1121/1.1643368 (2004).

148. Timmermans, M.-L., Toole, J., Krishfield, R. \& Winsor, P. Ice-Tethered Profiler observations of the double-diffusive staircase in the Canada Basin thermocline. Journal of Geophysical Research: Oceans 113. doi:https: / /doi . org/10 .1029/2008JC004829 (2008). 
149. Timmermans, M.-L., Cole, S. \& Toole, J. Horizontal Density Structure and Restratification of the Arctic Ocean Surface Layer.Journal of Physical Oceanography 42. doi:10 . 1175/JP0D-11-0125.1 (2012).

150. Timmermans, M.-L., Toole, J. \& Krishfield, R. Warming of the interior Arctic Ocean linked to sea ice losses at the basin margins. Science Advances 4. doi:10.1126/sciadv . aat6773 (2018).

151. Toole, J. M., Timmermans, M.-L., Perovich, D. K., Krishfield, R. A., Proshutinsky, A. \& Richter-Menge, J. A. Influences of the ocean surface mixed layer and thermohaline stratification on Arctic Sea ice in the central Canada Basin. Journal of Geophysical Research: Oceans 115. doi:https: //doi .org/10.1029/2009JC005660 (2010).

152. Toole, J., Krishfield, R., Timmermans, M.-L. \& Proshutinsky, A. The Ice-Tethered Profiler: Argo of the Arctic. Oceanography 24. doi:10.5670/oceanog. 2011.64 (2011).

153. Ulrich, K. T. \& Eppinger, S. D. Product design and development (2016).

154. Urban, C. D. Design and evaluation of a tactical decision aid in 1990IEEE International Conference on Systems, Man, and Cybernetics Conference Proceedings (1990). doi:10.1109/ICSMC . 1990.142233.

155. Urick, R. J. Principles of Underwater Sound, third edition Softcover edition (Peninsula Publishing, Los Altos, Calif, 2013).

156. Vaganay, J., Leonard, J., Curcio, J. \& Willcox, J. Experimental validation of the moving long base-line navigation concept in (2004). doi:10.1109/AUV . 2004.1431194.

157. Van Uffelen, L. J. V. Global Positioning Systems: Over Land and Under Sea. Acoustics Today 17 (2021).

158. Van Uffelen, L. J., Howe, B. M., Nosal, E.-M., Carter, G. S., Worcester, P. F. \& Dzieciuch, M. A. Localization and Subsurface Position Error Estimation of Gliders Using Broadband Acoustic Signals at Long Range. IEEE Journal of Oceanic Engineering 41. doi:10 . 1109 / JOE . 2015 . 2479016 (2016).

159. Van Uffelen, L. J., Nosal, E.-M., Howe, B. M., Carter, G. S., Worcester, P. F., Dzieciuch, M. A., Heaney, K. D., Campbell, R. L. \& Cross, P. S. Estimating uncertainty in subsurface glider position using transmissions from fixed acoustic tomography sources. The Journal of the Acoustical Society of America 134. doi:10.1121/1.4818841 (2013).

160. Verlinden, C. M. A., Sarkar, J., Hodgkiss, W. S., Kuperman, W. A. \& Sabra, K. G. Passive acoustic tracking using a library of nearby sources of opportunity. The Journal of the Acoustical Society of America 143. doi:10.1121/1.5022782 (2018).

161. Wagner, D. H. Naval Tactical Decision Aids 1989. 
162. Wåhlin, A. K., Graham, A. G. C., Hogan, K. A., Queste, B. Y., Boehme, L., Larter, R. D., Pettit, E. C., Wellner, J. \& Heywood, K. J. Pathways and modification of warm water flowing beneath Thwaites Ice Shelf, West Antarctica. Science Advances 7. doi:10 .1126/sciadv . abd7254 (2021).

163. Walton, J. Evolution of a Search System: Lessons Learned with the Advanced Unmanned Search System. 1992.

164. Wang, B., Zou, X. \& Zhu, J. Data assimilation and its applications. Proceedings of the National Academy of Sciences 97. doi:10 .1007/s00024-011-0388-x (2000).

165. Wang, D. Adaptive Rapid Environmental Assessment System Simulation Framework PhD Thesis (Massachusetts Institute of Technology, 2005).

166. Wang, D. Autonomous Underwater Vehicle Path Planning and Adaptive On-board Routing for Adaptive Rapid Environmental Assessment PhD Thesis (Massachusetts Institute of Technology, 2007).

167. Wang, D., Lermusiaux, P. F. J., Haley, P. J., Eickstedt, D., Leslie, W. G. \& Schmidt, H. Acoustically focused adaptive sampling and on-board routing for marine rapid environmental assessment. Journal of Marine Systems 78. doi:10 . 1016/ j . jmarsys . 2009 . 01 . 037 (2009).

168. Wang, M. \& Overland, J. E. A sea ice free summer Arctic within 30 years? Geophysical Research Letters 36. doi:https : / doi .org/10.1029/2009GL037820 (2009).

169. Webster, S. E., Freitag, L. E., Lee, C. M. \& Gobat, J. I. Towards real-time under-ice acoustic navigation at mesoscale ranges in 2015 IEEE International Conference on Robotics and Automation (ICRA) (2015). doi:10.1109/ICRA . 2015.7139231.

170. Webster, S. E., Eustice, R. M., Singh, H. \& Whitcomb, L. L. Advances in single-beacon oneway-travel-time acoustic navigation for underwater vehicles. The International Journal of Robotics Research 31. doi:10 .1177/0278364912446166 (2012).

171. Webster, S. E., Eustice, R. M., Singh, H. \& Whitcomb, L. L. Preliminary deep water results in single-beacon one-way-travel-time acoustic navigation for underwater vehicles. 2009 IEEE/RSJ International Conference on Intelligent Robots and Systems, IROS 2009. doi:10 . 1109/IROS . 2009. 5354457 (2009).

172. Widditsch, H. R. SPURV - The First Decade 1973.

173. Wilson, K. A Prototype Acoustic Battlefield Decision Aid Incorporating Atmospheric Effects and Arbitrary Sensor Layouts 1998.

174. Wilson, W. D. Speed of Sound in Sea Water as a Function of Temperature, Pressure, and Salinity. The Journal of the Acoustical Society of America 32. doi:10 . 1121 / 1 1908167 (1960).

175. Wu, L., Yang, X.-Y. \& Hu, J. Assessment of Arctic sea ice simulations in CMIP5 models. doi:10.5194/tc-2018-26 (2018). 
176. Wu, M., Barmin, M. P., Andrew, R. K., Weichman, P. B., White, A. W., Lavely, E. M., Dzieciuch, M. A., Mercer, J. A., Worcester, P. F. \& Ritzwoller, M. H. Deep water acoustic range estimation based on an ocean general circulation model: Application to PhilSea10 data. The Journal of the Acoustical Society of America 146. doi:10.1121/1. 5138606 (2019).

177. Xu, W. \& Schmidt, H. System-Orthogonal Functions for Sound Speed Profile Perturbation. IEEE Journal of Oceanic Engineering 31. doi:10 . 1109/ JOE. 2006.872221 (2006).

178. Yilmaz, N., Evangelinos, C., Lermusiaux, P. \& Patrikalakis, N. Path Planning of Autonomous Underwater Vehicles for Adaptive Sampling Using Mixed Integer Linear Programming. IEEE JOURNAL OF OCEANIC ENGINEERING 33. doi:10 . 1109/ JOE . 2008 . 2002105 (2008).

179. Yilmaz, N. K., Evangelinos, C., Patrikalakis, N. M., Lermusiaux, P. F. J., Haley, P. J., Leslie, W. G., Robinson, A. R., Wang, D. \& Schmidt, H. Path planning methods for adaptive sampling of environmental and acoustical ocean fields. Oceans 2006. doi:10.1109/0CEANS . 2006.306841 (2006).

180. Zhang, J., Weijer, W., Steele, M., Cheng, W., Verma, T. \& Veneziani, M. Labrador Sea freshening linked to Beaufort Gyre freshwater release. Nature Communications 12. doi:10 . 1038/s41467-021-21470-3 (2021).

181. Zhang, Y., Baggeroer, A. B. \& Bellingham, J. G. Spectral-Feature Classification of Oceanographic Processes Using an Autonomous Underwater Vehicle. IEEE Journal of Oceanic Engineering 26 (2001). 